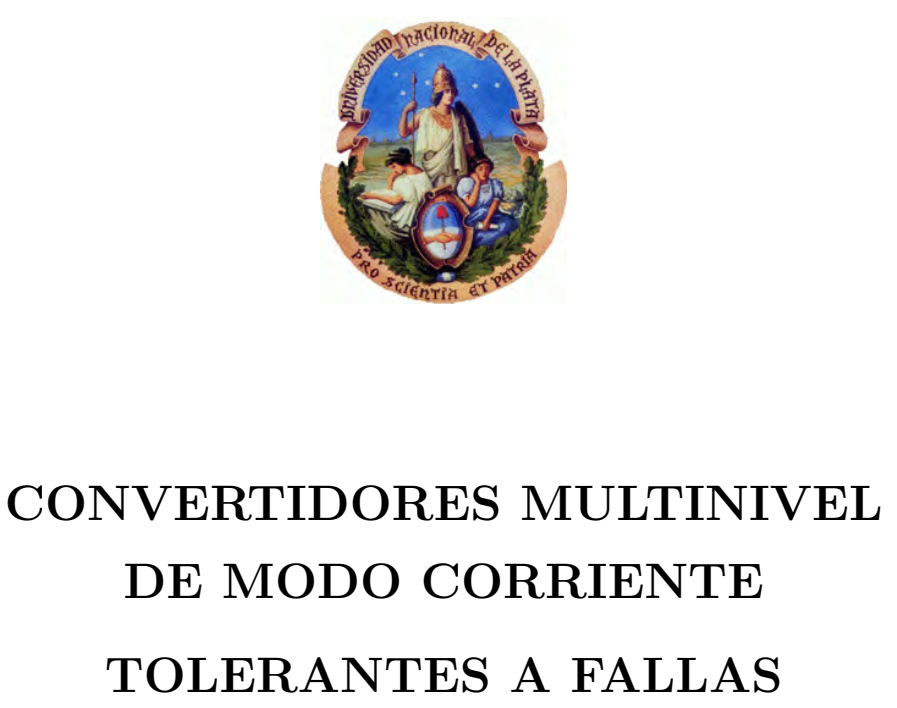

\title{
Tesis de Doctorado
}

MIGUEL PABLO AGUIRRE

Presentada ante la Facultad de Ingeniería de la

Universidad Nacional de la Plata

como requisito para la obtención del grado académico de

\section{DOCTOR EN INGENIERÍA}

Dirección de tesis:

Dra. María Inés Valla

Jurado de tesis:

Dr. Alejandro Raúl Oliva

Dr. Daniel Oscar Carrica

18 de diciembre de 2013 


\section{Resumen}

En esta tesis se realiza el estudio de los Convertidores Multinivel de Modo Corriente (MCSI), haciendo hincapié en la topología MCSI Simétrico o Single Rating Inductor MCSI por su especial característica modular que la hace ideal para aplicaciones industriales o de alta potencia. Se analiza en detalle el funcionamiento de la topología, incluyendo sus ventajas y desventajas en comparación con otras topologías de corriente y con los Convertidores Multinivel de Modo Tensión (MVSI). Se presenta el diseño de los circuitos y la estrategia de funcionamiento para conseguir un funcionamiento tolerante a fallas, mediante el agregado de un módulo de respaldo o hotspare. Se propone y analiza una la generación de las señales de disparo mediante una adaptación de la modulación de ancho de pulso por desplazamiento de portadoras (PSC-SPWM) y se demuestra que su uso genera un balance natural en las corrientes internas del convertidor. Se presentan dos técnicas de control para fortalecer el balance de corrientes frente a perturbaciones, que actúan sobre la modulación,con mínimos requerimientos de potencia de cálculo. Dado que los MCSI Simétricos son ideales para su uso en aplicaciones de alta potencia, se diseñan dos técnicas tendientes a minimizar las pérdidas en las llaves, reduciendo la cantidad de conmutaciones por ciclo y produciendo una conmutación suave de la corriente. Se demuestra, mediante simulaciones detalladas, el correcto funcionamiento de la topología en dos aplicaciones básica: a) como filtro activo para mejorar la calidad de los sistemas eléctricos, y b) como interfaz con sistemas de almacenamiento en base a hidrógeno. Finalmente se presentan resultados experimentales en un prototipo de laboratorio, utilizando una FPGA de alta velocidad para la generación de las señales de disparo y control. 


\section{Abstract}

In this thesis a comprehensive study of the Multilevel Current Source Inverters (MCSI) is performed, emphasizing on the Single Rating Inductor MCSI topology because of the modular configuration that makes it ideal for industrial and high power applications. The performance of the topology is analyzed and the pros\&cons against other current topologies as to the most common voltage (MVSI) topologies are presented. By adding a hot-spare module and a few switches Fault Tolerant operation is obtained, the sequence of events to keep the inverter behavior, after a fault is detected, is presented and analyzed. A proper implementation of the Phase-Shifted Carrier Sinusoidal Pulse Width Modulation (PSC-SPWM) is proposed and simulated, allowing a natural current balance in all modules. Two techniques for current balance enhancement are presented to avoid problems caused by disturbances, acting on the modulation with minimum computational cost. Since MCSI are suitable for high power applications, two techniques to reduce power losses are designed and presented: minimizing the number of commutations per cycle and soft switching features. The behavior of the topology in two iconic applications is thoroughly simulated: a) active filter to increase the power quality in electric systems, and b) to interface hydrogen generation and storage systems. Finally, experimental results are shown, by using a high speed FPGA to generate both the firing signals to the switches and the control algorithms. 


\section{Agradecimientos}

Durante el tiempo que trabajé en esta tesis y en general durante todo el curso de mi Doctorado recibí el apoyo de innumerables personas, a quienes estoy profundamente agradecido, pero a algunas de ellas quiero mencionar especialmente:

A mi mujer, Natalia Rey, y mi familia: mi mamá, mis dos hermanos y mi papá que me inspiró desde siempre. Al ITBA y sus autoridades, que me permitieron esta posibilidad. A quienes me ayudaron y apoyaron: Osvaldo Micheloud, Laura Calviño y Carolina Evangelista. A mis compañeros docentes: Héctor Polenta, Pablo Fierens, Pablo Cossutta y Ricardo Lauretta. A los alumnos que han pasado por el CIDEI y colaborado con el proyecto: Juan Matus, Alejandro Latini, J. P. Tettamanti, Mathías Angélico, José María Bracco. Un agradecimiento especial a todos los miembros del LEICI, que me han recibido y alentado en muchas oportunidades, especialmente a los que me tuvieron como alumno.

Y fundamentalmente a mi tutora, la Dra. Maria Inés Valla, quien durante estos años me ha enseñado mucho más de lo que imaginé, con una excelente calidad humana, siempre dispuesta a ayudar y aconsejar. 


\section{Abreviaturas}

$\begin{array}{ll}\text { AFC } & \text { Celda de Combustible Alcalina } \\ \text { APOD } & \text { Alternative Phase Opposition Disposition } \\ \text { CA } & \text { Corriente Alterna } \\ \text { CC } & \text { Corriente Continua } \\ \text { CSI } & \text { Current Source Inverter } \\ \text { DCMC } & \text { Diode Clamp Multilevel Converter } \\ \text { DSP } & \text { Digital Signal Processor } \\ E_{d} & \text { Tensión del Bus de Continua } \\ \text { EMI } & \text { Electro-Magnetic Interference } \\ \text { ETO } & \text { Emitter Turno-Off Tyristor } \\ \text { FPGA } & \text { Field Programmable Gate Array } \\ f s w & \text { Frecuencia de Conmutación } \\ I & \text { Corriente de la fuente Principal de un CSI } \\ I+D & \text { Investigación y Desarrollo } \\ \text { IGBT } & \text { Insulated Gate Bipolar Transistor } \\ \text { IGCT } & \text { Insulated Gate-Commutated Tyristor } \\ \mathrm{m}_{a} & \text { Índice de Modulación en Amplitud } \\ \text { MCSI } & \text { Multilevel Current Source Inverter } \\ \text { MOS } & \text { Metal Oxide Semiconductor Transistor } \\ \text { MTBF } & \text { Tiempo Medio Entre Fallas } \\ \text { MVSI } & \text { Multilevel Voltage Source Inverter } \\ \text { NPC } & \text { Neutral Point Clamped MVSI } \\ \text { PD } & \text { Phase Disposition } \\ & \end{array}$


PEMFC Membrana de Intercambio de Protones

PI Controlador Proporcional-Integral

PLL Lazo Enganchado de Fase

POD Phase Opposition Disposition

PSC Phase Shifted Carrier

PSC-SPWM Phase Shifted Carrier Sinusoidal PWM

PWM Pulse Width Modulation

SCR Silicon Controlled Rectifier

SOFC Celda de Combustible de Óxido Sólido

SPWM Sinusoidal Pulse Width Modulation

$t_{f v} \quad$ Tiempo de bajada de la tensión

THD $_{I} \quad$ Distorsión Total Armónica de Corriente

$\mathrm{THD}_{V} \quad$ Distorsión Total Armónica de Tensión

$t_{r i}$

Tiempo de subida de la corriente

TRIAC Triodo de Corriente Alterna

$V_{G S I o} \quad$ Tensión de compuerta de plena corriente

$V_{G S t h} \quad$ Tensión de compuerta de activación

VSI

Voltage Source Inverter 


\section{Índice general}

1. Introducción 1

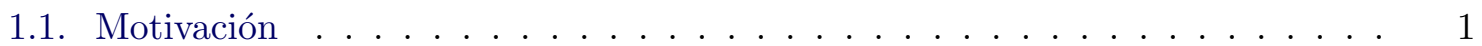

1.2. Antecedentes . . . . . . . . . . . . . . . . . . . 2

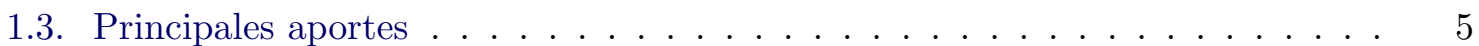

1.4. Organización y estructura de la Tesis . . . . . . . . . . . . . . . . . . 9

2. Convertidores Electrónicos de Potencia 11

2.1. Introducción . . . . . . . . . . . . . . . . . . . . . . . . . . . 11

2.2. Convertidores Multinivel Fuente de Tensión . . . . . . . . . . . . . . . . . . 13

2.2.1. Configuración en Cascada . . . . . . . . . . . . . . . . . . . . . . . 13

2.2.2. Capacitores Flotantes . . . . . . . . . . . . . . . . . . . . . 14

2.2.3. Enclavamiento por diodos . . . . . . . . . . . . . . . . . . . . 14

2.2.4. Configuración Híbrida . . . . . . . . . . . . . . . . . . . . . . 17

2.3. Convertidores Multinivel Fuente de Corriente monofásicos . . . . . . . . . . . 18

2.3.1. Obtención de topologías por el principio de dualidad V-I . . . . . . . . 18

2.3.2. Cascada . . . . . . . . . . . . . . . . . . . . . . . . 19

2.3.3. MCSI Asimétrico . . . . . . . . . . . . . . . . . . . . . . . . . 19

2.3.4. MCSI Simétrico . . . . . . . . . . . . . . . . . . 20

2.4. Convertidores Multinivel Fuente de Corriente polifásicos . . . . . . . . . . . . 21

2.4.1. MCSI en Cascada trifásicos . . . . . . . . . . . . . . . . . . 22

2.4.2. Multi Rating Inductor MCSI _. . . . . . . . . . . . . . . . . . . 22

2.4.3. Single Rating Inductor MCSI . . . . . . . . . . . . . . . . . . . . . . . 24

3. MCSI Simétrico Tolerante a Fallas 25

3.1. Introducción . . . . . . . . . . . . . . . . . . . . . . 25 


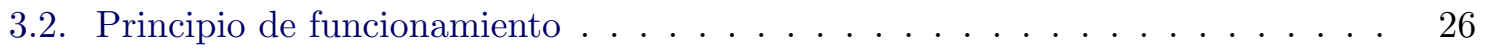

3.3. Modulación . . . . . . . . . . . . . . . . . . . . . . . 31

3.3.1. Tri-Logic SPWM . . . . . . . . . . . . . . . . 33

3.3.2. Estados cero . . . . . . . . . . . . . . . . 35

3.3.3. Minimización de la frecuencia de conmutación . . . . . . . . . . . . . 35

3.3.4. Modulación PSC-SPWM para un MCSI de tres módulos . . . . . . . . 38

3.3.5. Variación del índice de modulación . . . . . . . . . . . . . . . . . . . . 40

3.4. Eficiencia y Conmutación suave . . . . . . . . . . . . . . . . . . . . . 42

3.5. Tolerancia a fallas . . . . . . . . . . . . . . . . . . . . 45

3.5.1. Tolerancia a fallas en el MCSI Simétrico . . . . . . . . . . . . . . . . 46

3.6. Balance de corrientes . . . . . . . . . . . . . . . . . . . . . . 50

3.6.1. Control por desplazamiento de fase . . . . . . . . . . . . . . . . 52

3.6.2. Control por amplitud de portadora . . . . . . . . . . . . . . . . . 57

3.6.3. Modulación en FPGA . . . . . . . . . . . . . . . . . . . . . . . . 60

3.6.4. Formas de onda experimentales . . . . . . . . . . . . . . . . . . 64

3.7. Conclusiones . . . . . . . . . . . . . . . . . . . . . . . . 69

$\begin{array}{ll}\text { 4. Aplicaciones } & 71\end{array}$

4.1. Interfaz para almacenamiento de energia en forma de $H_{2} \ldots \ldots \ldots \ldots$. . . . 72

4.1.1. Electrolizadores . . . . . . . . . . . . . . . . . . . 72

4.1.2. Celdas de combustible. . . . . . . . . . . . . . . . . . . 73

4.1.3. Interfaz con el sistema de almacenamiento de $H_{2} \ldots \ldots \ldots$

4.2. Acondicionamiento de redes eléctricas . . . . . . . . . . . . . . 81

4.2.1. Sistema Eléctrico Modelo . . . . . . . . . . . . . . . . . . . . . 82

4.2.2. Filtro Activo . . . . . . . . . . . . . . . . . . 83

4.2.3. Compensación de factor de potencia . . . . . . . . . . . . . . . . 88

4.3. Conclusiones . . . . . . . . . . . . . . . . . . . . . . . . 91

$\begin{array}{lr}\text { 5. Conclusiones } & 93\end{array}$

5.1. Conclusiones . . . . . . . . . . . . . . . . . . . . 93

5.2. Trabajos a futuro . . . . . . . . . . . . . . 96

$\begin{array}{ll}\text { A. Diseño del prototipo } & 99\end{array}$

A.1. Fuente de corriente principal _. . . . . . . . . . . . . . . . . . . 100

A.2. Banco de capacitores . . . . . . . . . . . . . . . . . . . . . . . 101 
A.3. Circuitos de disparo . . . . . . . . . . . . . . . . . . . . . . . . . 101

A.4. Carga trifásica variable . . . . . . . . . . . . . . . . . 105

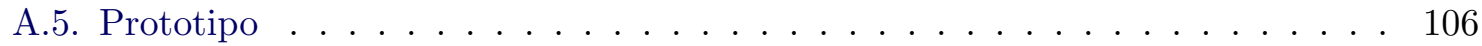




\section{Índice de figuras}

2.1. Comparación entre formas de onda: CSI y MCSI. . . . . . . . . . . . . . . . 12

2.2. MVSI cascada y capacitores flotantes, monofásicos de cinco niveles . . . . . . 14

2.3. MVSI de enclavamiento por diodos monofásico de cinco niveles. . . . . . . . . 15

2.4. MVSI DCMC trifásico de cinco niveles . . . . . . . . . . . . . . . . . 16

2.5. DCMC en conexión back-to-back . . . . . . . . . . . . . . . 17

2.6. MVSI de configuración híbrida de cinco niveles. . . . . . . . . . . . . . . . 17

2.7. Dualidad Tensión-Corriente: llaves de potencia . . . . . . . . . . . . . . . . . 19

2.8. MCSI cascada monofásico. . . . . . . . . . . . . . . . . . . . . . . . . . 19

2.9. MCSI Asimétrico monofásico de cinco niveles . . . . . . . . . . . . . . . . 20

2.10. MCSI Simétrico monofásico de cinco niveles. . . . . . . . . . . . . . . . . 21

2.11. MCSI cascada trifásico de cinco niveles . . . . . . . . . . . . . . . . . . . 22

2.12. MCSI Asimétrico trifásico de nueve niveles . . . . . . . . . . . . . . . . 23

2.13. MCSI Simétrico trifásico de nueve niveles . . . . . . . . . . . . . . . . . 24

3.1. Conexión de convertidores MVSI y MCSI _ . . . . . . . . . . . 26

3.2. Circuito del convertidor MCSI Simétrico . . . . . . . . . . . . . . . . . 27

3.3. Conmutación de corrientes en un módulo MCSI Simétrico . . . . . . . . . . 28

3.4. Corriente de salida multinivel mediante conmutación por tabla . . . . . . . . 29

3.5. Configuración del circuito para distintas corrientes de salida . . . . . . . . . 30

3.6. Configraciones equivalentes: redundancia de estados . . . . . . . . . . . . . 30

3.7. Modulación de convertidores multinivel . . . . . . . . . . . . . . . . . . 31

3.8. Modulación SPWM y Tri-Logic . . . . . . . . . . . . . . . . . . . . 34

3.9. Redundancia de estados cero . . . . . . . . . . . . . . . . . . 35

3.10. Máquina secuencial para la selección del estado cero óptimo . . . . . . . . . . 37 
3.11. Minimización del número de conmutaciones por ciclo . . . . . . . . . . . . . . 39

3.12. Modulación PSC-SPWM . . . . . . . . . . . . . . . . . . . . . . . . . 39

3.13. Modulación PSC-SPWM para un MCSI de tres módulos . . . . . . . . . . . . 40

3.14. Variación del punto de trabajo . . . . . . . . . . . . . . . . . . 41

3.15. Conmutación suave . . . . . . . . . . . . . . . . . . . . . . . . 44

3.16. MCSI Simétrico tolerante a fallas . . . . . . . . . . . . . . . . . . . 46

3.17. Operación tolerante a fallas, primer etapa . . . . . . . . . . . . . . 47

3.18. Operación tolerante a fallas, segunda etapa . . . . . . . . . . . . . . 47

3.19. Operación tolerante a fallas, tercer etapa . . . . . . . . . . . . . 48

3.20. Operación tolerante a fallas, cuarta etapa . . . . . . . . . . . . . . 49

3.21. Efecto de la operación tolerante a fallas en la salida . . . . . . . . . . . 50

3.22. Balance natural de corrientes producido por la modulación PSC-SPWM . . . 51

3.23. Control de balance, principios básicos . . . . . . . . . . . . . 52

3.24. Efecto de cambios en la fase de portadoras . . . . . . . . . . . . . . 52

3.25. Detalle del efecto de cambio en la fase de portadoras . . . . . . . . . . . . 54

3.26. Control de balance por fase de portadoras . . . . . . . . . . . . . . 55

3.27. Control de balance por cambio de fase a lazo cerrado . . . . . . . . . . . . 56

3.28. Efecto del control de balance en el espectro de salida . . . . . . . . . . . . . . 56

3.29. Control por amplitud de portadoras, ganancia variable . . . . . . . . . 57

3.30. Efecto de cambios en amplitud de portadoras . . . . . . . . . . . . . 58

3.31. Detalle del efecto de cambio en amplitud de portadoras . . . . . . . . . . 58

3.32. Control de balance por amplitud de portadoras . . . . . . . . . . . . . . 60

3.33. Control de balance por cambio de amplitud a lazo cerrado . . . . . . . . . . . 61

3.34. Efecto del control de balance por amplitud en el espectro de salida . . . . . . 61

3.35. Modulación en FPGA . . . . . . . . . . . . . . . . . . . . . . . 62

3.36. Resultados experimentales . . . . . . . . . . . . . . . . 65

3.37. Resultados experimentales: detalle de corriente de salida y tensión en una llave 66

3.38. Resultados experimentales: carga inductiva . . . . . . . . . . . 67

3.39. Resultados experimentales: respuesta dinámica frente a un salto de carga . . 67

3.40. Resultados experimentales: respuesta dinámica, detalle . . . . . . . . . . . . 68

3.41. Resultados experimentales: respuesta dinámica, generación de armónicas . . . 68

4.1. Curva característica tensión vs. corriente del electrolizador de alta presión 73 
4.2. Diagrama esquemático de una celda de combustible . . . . . . . . . . . . . . . 74

4.3. Característica tensión vs. corriente de una celda de combustible PEMFC típica 74

4.4. Interfaz con almacenamiento de hidrógeno . . . . . . . . . . . . . . . . . 75

4.5. Bloque de control . . . . . . . . . . . . . . . . . . . . . . 76

4.6. Flujo de energía en el sistema . . . . . . . . . . . . . . . . . . . 76

4.7. Simulación del comportamiento del sistema ante situaciones típicas . . . . . . 77

4.8. Conexión del electrolizador y el MCSI . . . . . . . . . . . . . . . . . 78

4.9. Intercambios de potencia con el electrolizador . . . . . . . . . . . . . . . . 79

4.10. Conexión del electrolizador y el MCSI con potencia reactiva . . . . . . . . . . 79

4.11. Conexión de la celda de combustible y el MCSI _ . . . . . . . . . . . . . . 80

4.12. Intercambios de potencia con la celda de combustible . . . . . . . . . . . . 80

4.13. Conexión de la celda de combustible y el MCSI con potencia reactiva . . . . . 81

4.14. Modelo de sistema eléctrico . . . . . . . . . . . . . . . . . . . 82

4.15. Espectros de tensión y corriente del sistema sin compensar . . . . . . . . . . . 84

4.16. Conexionado del filtro activo . . . . . . . . . . . . . . . . . 84

4.17. Control del MCSI . . . . . . . . . . . . . . . . . . . . . 86

4.18. Formas de onda durante el arranque del filtro activo . . . . . . . . . . . 87

4.19. Espectro de tensión resultante de la compensación . . . . . . . . . . . . . 88

4.20. Espectro de corriente resultante de la compensación . . . . . . . . . . . 88

4.21. Respuesta dinámica frente a un cambio de carga . . . . . . . . . . . . . 89

4.22. Intercambio de potencias: efectos del filtro activo y salto de carga . . . . . . . 90

4.23. Forma de onda de corriente de salida del MCSI . . . . . . . . . . . . . . . . 91

A.1. Esquema general del prototipo . . . . . . . . . . . . . . . . . . 99

A.2. Fuente principal: placa de circuito impreso . . . . . . . . . . . . . . . . 101

A.3. Disparo de los IGBT . . . . . . . . . . . . . . . . . . . . 102

A.4. Señales de compuerta de los IGBT . . . . . . . . . . . . . . . . . 103

A.5. Señales de compuerta de los IGBT: tiempos caracteristicos . . . . . . . . . . . 104

A.6. Conexionado de un módulo MCSI Simétrico . . . . . . . . . . . . . . . . . 105

A.7. Vista general del arreglo de laboratorio para conexionado y pruebas. . . . . . 106

A.8. Detalle del prototipo y la fuente de CC de laboratorio. . . . . . . . . . . . . 107

A.9. Sistema de adquisición de datos y FPGA de control. . . . . . . . . . . . . . 107

A.10.Detalle del conexionado de los módulos. . . . . . . . . . . . . . . . . . . 108 
A.11.Detalle de un módulo. . . . . . . . . . . . . . . . . . . . . . . 108

A.12.Vista de los inductores divisores y principales. . . . . . . . . . . . . 109 


\section{Capítulo 1}

\section{Introducción}

\subsection{Motivación}

La creciente demanda de energía, por parte de la sociedad en conjunto, requiere el continuo desarrollo de nuevas tecnologías a fin de perfeccionar las características de los generadores, los convertidores y las cargas. Esto implica maximizar el rendimiento, disminuir las posibilidades de fallas, facilitar las tareas de mantenimiento y prevención, entre otras mejoras posibles. Por ello es indispensable contar con los conocimientos necesarios para la construcción y operación segura de convertidores de cada vez mayor potencia y confiabilidad y con mejores prestaciones desde el punto de vista de la respuesta dinámica y la calidad de las formas de onda de salida.

Por otro lado, la inyección de gran cantidad de energía generada por fuentes renovables provee una solución provisoria frente a la futura carencia de combustibles fósiles, pero crea un enorme problema de estabilidad y calidad de energía en los sistemas eléctricos en los que es aplicada. Las nuevas tecnologías de generación, basadas en energías renovables, o limpias, exigen sistemas de conversión y control en medias y altas potencias, con una buena respuesta dinámica y velocidad de reacción. Además, el rendimiento de todo el sistema debe maximizarse para permitir su utilización en situaciones de competencia económica que todavía les son desfavorables, pero que son indispensables para obtener la experiencia práctica que el desarrollo futuro requiere. Pero, la inyección de gran cantidad de energía renovable en un sistema eléctrico, lleva a diversos problemas de estabilidad y control, especialmente porque la generación suele presentar fuertes variaciones debido a la dependencia de las condiciones naturales. A su vez, la generación convencional basada en sistemas mecánicos presenta tiempos característicos muy lentos y la combinación de ambos sistemas en lo denominado como Smart Grids conlleva enormes problemas de estabilidad y seguridad, algunos de los cuales pueden ser mitigados mediante nuevas tecnologías en sistemas de almacenamiento, como baterías de flujo, generación de hidrógeno y otras, pero para poder operar un sistema de estas características, con algoritmos de control muy complejos, es indispensable la utilización de convertidores electrónicos de potencia. 
Los convertidores electrónicos de potencia juegan un papel preponderante en la utilización de la energía eléctrica a todo nivel, tanto en baja potencia, como por ejemplo en la alimentación de pequeños dispositivos, como en alta potencia en la interconexión y/o control de sistemas eléctricos y grandes motores industriales. Además, las nuevas tecnologías de llaves y circuitos de control permiten el desarrollo de convertidores con mejores prestaciones, que incluyen un rango mucho mayor de potencias y frecuencias de operación, menor contenido armónico en su salida y, sobre todo, mayor confiabilidad basada en estructuras tolerantes a fallas. Las razones expuestas permiten afirmar que el desarrollo de nuevas topologías de convertidores es vital para poder adaptarse a los requerimientos cada vez más exigentes de toda la cadena entre la generación y el consumo de energía eléctrica, pasando por el transporte, la distribución, el acondicionamiento de los sistemas eléctricos, la supresión de distorsiones y/o asimetrías y la transformación entre niveles de tensión, corriente y frecuencia, e incluso entre corriente alterna y continua. La investigación en los diferentes esquemas circuitales y su funcionamiento, las técnicas de control y tecnologías asociadas es un área de investigación plena de desafiantes problemas desde el punto de vista práctico y también, fundamentalmente, un campo de $I+D$ científico-tecnológico de gran interés por razones ambientales, sociales, económicas y estratégicas.

\subsection{Antecedentes}

Los convertidores electrónicos se utilizan en la actualidad en una gran variedad de aplicaciones en todo el espectro de potencias, desde pequeñas fuentes de alimentación (Powerint, 2009; Al-Hanai et al., 2011) hasta enormes convertidores para aplicaciones eléctricas (Hingorani y Gyugyi, 2000; Corasaniti et al., 2008; Adam et al., 2013). Si bien las topologías básicas se mantienen vigentes, los avances en tecnologías de llaves, circuitos y métodos de control y el desarrollo de nuevas tecnologías en fuentes de energía han permitido el desarrollo de nuevas topologías con mejores prestaciones que los convertidores tradicionales (Luo y Ye, 2005). Aunque tradicionalmente se utilizan los términos inversores, para aquellas aplicaciones donde se entrega energía al lado de CA, y rectificadores, para aquellas otras en las que se transfiere energía del lado de CA al de CC, se considera más adecuando el término convertidores para los circuitos utilizados en la actualidad, ya que el uso técnicas de modulación y control avanzadas permiten el flujo de potencia instantánea en ambos sentidos simultáneamente (Akagi et al., 2007).

En el caso de los convertidores de potencia con salida sinusoidal polifásica, las estructuras tradicionales de tensión de dos niveles se mantienen vigentes, lo que no ocurre con los convertidores de corriente. Los CSI (Current Source Inverter) de tres niveles fueron muy utilizados en un principio (Kim et al., 1984; Hombu et al., 1985; Nonaka y Neba, 1987; Espinoza y Joos, 1993; Zargari et al., 1997), gracias a los métodos de autoapagado de los SCR, pero luego perdieron mercado frente a los VSI (Voltage Source Inverter) debido al avance de los transistores de potencia y al desarrollo de nuevas técnicas de modulación y control. Tal es así que es posible encontrar innumerable cantidad de libros sobre convertidores de tensión y sus aplicaciones, pero prácticamente ninguno sobre convertidores fuente de corriente 
(Hingorani y Gyugyi, 2000; Mohan et al., 2005; Holmes y Lipo, 2003; Erickson y Maksimovic, 2012; Valla et al., 2013).

No obstante, los CSI se utilizan actualmente en convertidores como filtros activos y para el control de motores de muy alta potencia (9.000HP) debido a las ventajas propias de la topología, como control directo de corriente, robustez frente a cortocircuitos, eficiencia y vida útil de sus componentes, entre otras (Ishikawa y Murai, 2000; Beig y Ranganathan, 2006; Suh et al., 2007; Bai et al., 2011; Terciyanli et al., 2012). Pero su uso genera grandes inconvenientes debido a la interferencia electromagnética (EMI) que generan y los ripples de corriente y tensión en su salida. Por otro lado, el desarrollo de nuevas tecnologías de llaves y métodos de control de los últimos años, sumado al aumento de la demanda de convertidores de mayor potencia y mejor confiabilidad, han motivado la investigación en nuevas topologías de convertidores CSI, que utilizando las ventajas de una salida de múltiples niveles, pueda ser utilizado en aplicaciones cada vez más demandantes en cuanto a potencia y prestaciones. Es así que en los últimos años han comenzado a aparecer trabajos que analizan distintas topologías multinivel de CSI (Kwak y Toliyat, 2006; Gao et al., 2010; Suroso y Noguchi, 2011; Suresh y Panda, 2012; Dupczak et al., 2012; Suroso y Noguchi, 2012), cuyas características particulares los hacen especiales para ciertas aplicaciones, incluso superando a los Convertidores Multinivel Fuente de Tensión (MVSI) en potencia y prestaciones.

Una alternativa de diseño de topologías Convertidores Multinivel Fuente de Corriente (MCSI) utilizada en la bibliografía consiste en emplear el principio de dualidad TensiónCorriente, partiendo de las topologías conocidas de tensión, para obtener circuitos duales de corriente (Bai y Zhang, 2008a). De esta manera se pretende extrapolar el knowhow obtenido para la topología MVSI original y así simplificar el análisis y diseño del convertidor dual (McGrath y Holmes, 2008a; Zmood y Holmes, 1998). Para esto es necesario realizar un relevamiento de las topologías MVSI utilizadas actualmente, sus características de funcionamiento, ventajas y desventajas, a fin de compararlas con los requerimientos específicos de cada aplicación y así obtener una solución adecuada. Analizando la bibliografía se identifican cuatro principales topologías de convertidores MVSI: cascada (Dixon y Moran, 2002; Ertl et al., 2002; Malinowski et al., 2010), enclavamiento por diodos (Nabae et al., 1981; Rodriguez et al., 2010; Verne y Valla, 2010a; Ewanchuk et al., 2011), capacitores flotantes y topologías híbridas (Rech y Pinheiro, 2007; Gonzalez et al., 2010), cada una de ellas con sus particulares características de funcionamiento (Rodriguez et al., 2002, 2007; Franquelo et al., 2008; Lai y Peng, 1996; Rodriguez et al., 2009; Peng et al., 2010). Por otro lado, si bien es posible obtener topologías de corriente mediante el principio de dualidad Tensión-Corriente, no siempre el resultado es favorable en cuanto a prestaciones o estructura circuital resultante. Afortunadamente se han desarrollado otras topologías de MCSI con excelentes prestaciones, pero que no se corresponden con ningún convertidor MVSI ya conocido por lo que su análisis no puede realizarse a través de extrapolar otros resultados. Estas nuevas topologías requieren un estudio detallado de sus características, formas de operación y control y prestaciones (Xiong et al., 2004a,b; Bai et al., 2008).

La confiabilidad de los convertidores de alta potencia resulta vital, por un lado para garantizar la operación de las aplicaciones en los que se utilizan, sobre todo en sistemas 
eléctricos; y por otro lado por la cantidad de energía involucrada, que en caso de fallas puede generar daños físicos considerables tanto a equipos como a personas (Yang et al., 2011a; Rodriguez et al., 2009; Yang et al., 2011b). Es por ello que el desarrollo de convertidores y técnicas de operación tolerantes a fallas es vital para un uso seguro en aplicaciones de altas potencias, a fin de garantizar el funcionamiento continuo a pesar de una falla (Kou et al., 2004; Welchko et al., 2004; Li y Xu, 2006; Ma et al., 2007; Cordeiro et al., 2011). El uso de topologías y convertidores en forma modular permite además la implementación de técnicas de control preventivo, el ciclado de componentes y la instalación de módulos de respaldo en caliente o hot-spare, para reemplazar una parte dañada sin detener la operación del convertidor, ni reducir sus prestaciones (Correa et al., 2006).

Otra característica importante al momento de evaluar distintas topologías de convertidores de potencia es la implementación de los algoritmos de modulación, control y sincronización (Wang y Ooi, 1993; Lakshminarayanan et al., 2007; Hoffmann et al., 2011; Bai y Zhang, 2008b; Ciobotaru et al., 2008; Lu et al., 2013), ya que las distintas tecnologías disponibles en la actualidad, microcontroladores, DSP y FPGA, presentan características disímiles. Por ello deben evaluarse en profundidad para obtener la mejor solución para cada aplicación, debiendo considerarse, entre otras: la confiabilidad y robustez frente a perturbaciones, la velocidad de procesamiento, la disponibilidad en el mercado, facilidad de actualización en el tiempo, capacidad de procesamiento en paralelo, posibilidades de comunicación con otros dispositivos y la flexibilidad para la implementación de algoritmos de distintas características: operaciones en punto fijo y/o flotante, lógica libre de glitches, multiplicaciones y divisiones rápidas, etc.

El balance de tensión o corriente de los elementos de almacenamiento interno de los convertidores multinivel es un tema que requiere un dedicado análisis, ya que afecta directamente a la operación, confiabilidad y control de todo el convertidor. En el caso de las topologías fuente de tensión existen en al literatura numerosos trabajos que tratan el tema, tanto desde el punto de vista práctico, como teórico o matemático (Marchesoni et al., 1998; Peng, 2001; Marchesoni y Tenca, 2002; McGrath y Holmes, 2008b; Ceballos et al., 2011). Lamentablemente el tema del balance de la corriente en los inductores divisores de los convertidores MCSI no ha sido tratado prácticamente en la literatura y poca información existe al respecto.

Gracias a las tecnologías de llaves electrónicas de potencia disponibles en estos momentos (Li et al., 2009; Dijkhuizen y Norrga, 2011), no hay diferencias sustanciales en la operación de convertidores fuente de tensión o corriente, por lo cual es importante actualizar el conocimiento de los convertidores fuente de corriente a fin de permitir el uso de la tecnología en aquellas aplicaciones donde presentan evidentes ventajas frente a los convertidores fuente de tensión. Como conclusión de este análisis, motiva la realización de la presente tesis la necesidad de generar el conocimiento necesario para el diseño y operación de un convertidor fuente de corriente, con operación tolerante a fallas y excelentes prestaciones dinámicas. 


\subsection{Principales aportes}

En este trabajo se realizó el estudio de los aspectos fundamentales de los convertidores multinivel fuente de corriente simétricos o Single Rating Inductor MCSI, como ser la modulación, su operación tolerante a fallas, el balance de las corrientes internas, su aplicación en tres casos concretos: como filtro activo en sistemas eléctricos, como interfaz con energías alternativas y para la producción de hidrógeno. Se enfocó el estudio en un convertidor de tres módulos, con la posibilidad de generar siete niveles en la corriente de salida, cuya complejidad permite un análisis detallado y obtener conclusiones sin perder generalidad, por lo que los resultados son extrapolables a cualquier número de módulos. Los conocimientos adquiridos permitieron construir un prototipo de $3 \mathrm{KW}$ en el que se verificaron prácticamente los principales conceptos teóricos.

En forma detallada los principales aportes de esta tesis pueden resumirse en los siguientes temas:

- Modulación: se adaptaron dos técnicas de modulación utilizadas en convertidores multinivel de tensión: Modulación por Ancho de Pulso con Portadoras Desplazadas o Phase Shifted Carrier Sinusoidal PWM (PSC-SPWM) y Tri-Logic PWM. La combinación de estas técnicas produce las señales de disparo adecuadas para un correcto funcionamiento del convertidor, con excelente respuesta dinámica y mínima potencia de cálculo. La aplicación de la modulación PSC-SPWM produce además un balance de las corrientes internas del convertidor, que si bien es débil frente a perturbaciones y posee un pequeño error en estado estacionario, es suficiente para su estudio básico. Por otro lado, haciendo uso de la redundancia de estados cero de cada módulo del convertidor, se diseñó una estrategia para identificar los estados óptimos que permitan minimizar la cantidad de conmutaciones por ciclo de las llaves. De esta manera se consigue minimizar la potencia disipada, las emisiones electromagnéticas (EMI) y los requerimientos de los circuitos de disparo. La lógica resultante se implementa mediante una máquina de estados secuencial que detecta el punto de funcionamiento del convertidor y asigna a las llaves el estado cero óptimo en cada momento. Los resultados han sido publicados en los siguientes trabajos:

- Aguirre M., Calviño L., Valla M.I., "Multilevel Current Source Inverter with FPGA Control". IEEE Transactions on Industrial Electronics, vol.60, no.1, pp.3-10, Enero 2013, doi: 10.1109/TIE.2012.2185014.

○ M.P. Aguirre, M.L. Calviño, M.I. Valla, "Fault Tolerant Multilevel Current Source Inverter". IEEE International Conference on Industrial technology, ICIT2010 . Viña del Mar, Chile, Marzo 2010.

- M.P. Aguirre, M.L. Calviño, F. Corasaniti, M.I. Valla, "Multilevel Current Source Inverter to Improve Power Quality in a Distribution Network". IEEE International Symposium on Industrial Electronics, ISIE2010. Bari, Italia, Julio 2010.

- Minimización de las pérdidas por conmutación: mediante el análisis de la forma de 
conmutación de las llaves de cada módulo del convertidor se logró identificar la forma de producir una conmutación "suave" de la corriente entre dos llaves consecutivas. De esta manera se consigue minimizar las pérdidas por conmutación, maximizando el rendimiento y permitiendo su aplicación en convertidores de muy alta potencia. A modo de ejemplo, la disipación de los IGBT del prototipo es casi nula, no requiriendo de disipadores a pesar de entregar $3 \mathrm{KW}$ reales a la carga. Los principales resultados han sido publicados en el siguiente trabajo:

- Aguirre M., Calviño L., Valla M.I., "Multilevel Current Source Inverter with FPGA Control". IEEE Transactions on Industrial Electronics, vol.60, no.1, pp.3-10, Enero 2013, doi: 10.1109/TIE.2012.2185014.

- Tolerancia a fallas: se desarrolló una técnica para garantizar el funcionamiento del convertidor luego de la presencia de una falla en cualquiera de sus componentes internos. Esto se logra mediante el agregado de un módulo de respaldo en caliente (Hot-Spare) y los circuitos necesarios para la desconexión del módulo bajo falla en forma segura y recuperar la energía almacenada en sus inductores. Se simuló la estructura propuesta y los resultados han sido publicados en los siguientes trabajos:

- M.P. Aguirre, L. Slobozian, M.L. Calviño, M.I. Valla "Protección contra fallas en Convertidores de Modo Corriente". XIII RPIC - Reunión de Trabajo en Procesamiento de la Información y Control. Rosario, Argentina, Septiembre 2009.

- M.P. Aguirre, M.L. Calviño, M.I. Valla, "Fault Tolerant Multilevel Current Source Inverter". IEEE International Conference on Industrial technology, ICIT2010 . Viña del Mar, Chile, Marzo 2010.

- Implementación de la lógica de control mediante FPGA: las lógicas de modulación, de minimización del número de conmutaciones por ciclo y de control de balance de corrientes han sido adaptadas para su implementación en una FPGA comercial tipo Spartan 3E de Xilinx. De esta manera se consigue optimizar los tiempos de procesamiento y aprovechar la modularidad de la topología para realizar el procesamiento de las señales de modulación, control, balance de corrientes y disparo de las llaves en forma paralela, minimizando los retardos y tiempos de cálculo. Los resultados han sido publicados en los siguientes trabajos:

- Aguirre M., Calviño L., Valla M.I., "Multilevel Current Source Inverter with FPGA Control". IEEE Transactions on Industrial Electronics, vol.60, no.1, pp.3-10, Enero 2013, doi: 10.1109/TIE.2012.2185014.

○ J.P. Tettamanti, A.R. Latini, M.P. Aguirre, "An Example of Rapid Design of Power Electronics Control with Fpga in Matlab/Simulink". VI Southern Programmable Logic Conference - SPL2011. Córdoba, Argentina, Abril 2011.

- M.P. Aguirre, P. Cossutta, J.M. Bracco, M.I. Valla, "Sincronización con la red de un Convertidor Multinivel Fuente de Corriente con control de balance para 
generación fotovoltaica". XV RPIC - Reunión de Trabajo en Procesamiento de la Información y Control. Bariloche, Río Negro, Argentina, Septiembre 2013.

- Control del balance de corrientes: se diseñaron dos estrategias de control para garantizar el balance de las corrientes internas frente a perturbaciones y desbalances. Ambas se basan en introducir pequeñas variaciones en las portadoras de la modulación PSCSPWM para modificar el valor medio de las corrientes internas del convertidor a voluntad, minimizando el error en estado estacionario mediante la utilización de simples controladores PI tradicionales. Los resultados han sido publicados en los siguientes trabajos:

- M.P. Aguirre, J.M. Bracco, M. Angélico Engelhardt, M.I. Valla, "Balance de Corrientes en un Convertidor Multinivel Fuente de Corriente". XXIII ${ }^{\circ}$ Congreso Argentino de Control Automático, AADECA 2012. Buenos Aires, Argentina, Octubre 2012.

- M.P. Aguirre, M.I. Valla, "Current Balance Control in a Multilevel CurrentSource Inverter". International Conference on Industrial Electronics - ICIT 2013. Cape Town, SouthAfrica, Febrero 2013.

- M.P. Aguirre, P. Cossutta, J.M. Bracco, M.I. Valla, "Sincronización con la red de un Convertidor Multinivel Fuente de Corriente con control de balance para generación fotovoltaica". XV RPIC - Reunión de Trabajo en Procesamiento de la Información y Control. Bariloche, Río Negro, Argentina, Septiembre 2013.

- Aplicación a sistemas eléctricos: se simuló y analizó la aplicación de la topología bajo estudio en sistemas eléctricos de potencia, verificando su excelente respuesta tanto para la corrección de factor de potencia como para la realización de un filtro activo para la compensación de armónicas, distorsiones y desbalances. Mediante simulaciones detalladas, a nivel transistor, y utilizando las lógicas de control real a ser implementadas en la FPGA, se verificó que el convertidor presenta excelentes prestaciones en cuanto a respuesta dinámica, pudiendo compensar armónicas de alta frecuencia manteniendo la frecuencia de conmutación de las llaves dentro de los límites permitidos por la tecnología de componentes de potencia actuales. Los principales resultados han sido publicados en los siguientes trabajos:

- M.P. Aguirre, M.L. Calviño, F. Corasaniti, M.I. Valla, "Multilevel Current Source Inverter to Improve Power Quality in a Distribution Network". IEEE International Symposium on Industrial Electronics, ISIE2010. Bari, Italia, Julio 2010.

- M.P. Aguirre, M.L. Calviño, F. Corasaniti, M.I. Valla, "Filtro Activo De Modo Corriente Multinivel Para Mejorar La Calidad De Potencia En Sistemas De Distribución". XXII Congreso Argentino de Control Automático, AADECA 2010. Buenos Aires, Argentina, Agosto/Septiembre 2010.

- Aguirre M., Couto H., Valla M.I., "Analysis and simulation of a hydrogen based electric system to improve power quality in distributed grids". International 
Journal of Hydrogen Energy, Volume 37, Issue 19, October 2012, Pages 1495914965, doi: 10.1016/j.ijhydene.2012.01.163.

- Interfaz con energías alternativas: se simuló y analizó la aplicación de la topología como interfaz con sistemas de energías alternativas, en especial con sistemas fotovoltaicos y la producción y almacenamiento de hidrógeno. Se verificó el correcto funcionamiento del convertidor en simulación de aplicaciones reales, tomando ventaja del hecho de comportarse como fuente de corriente del lado del bus de continua, permitiendo una interfaz directa tanto con los sistemas de paneles solares, como con los electrolizadores y celdas de combustible. De esta manera se puede prescindir de las etapas intermedias tipo elevadoras (boost), maximizando el rendimiento y la confiabilidad al reducir el número de llaves y los puntos de falla. Los resultados han sido publicados en los siguientes trabajos:

○ Aguirre M., Couto H., Valla M.I., "Analysis and simulation of a hydrogen based electric system to improve power quality in distributed grids". International Journal of Hydrogen Energy, Volume 37, Issue 19, October 2012, Pages 1495914965, doi: 10.1016/j.ijhydene.2012.01.163

- M.P. Aguirre, A.R. Latini, H.P. Couto, M.I. Valla, "Análisis y Simulación de un Sistema Ininterrumpido de Energía Mediante Almacenamiento de Hidrógeno". $4^{\circ}$ Congreso Nacional - $3^{\circ}$ Congreso Iberoamericano Hidrógeno y Fuentes Sustentables de Energía - HYFUSEN 2011. Mar del Plata, Argentina, Junio 2011.

- M.P. Aguirre, M.I. Valla, "An environmental friendly alternative for hydrogen production and electric energy generation". 37th Annual Conference on IEEE Industrial Electronics Society - IECON 2011. Melbourne, Australia, Noviembre 2011.

○ M.P. Aguirre, A.R. Latini, H.P. Couto, M.I. Valla, "Convertidor Bidireccional para la Producción de Hidrógeno y Generación de Electricidad". XIV RPIC - Reunión de Trabajo en Procesamiento de la Información y Control. Oro Verde, Entre Ríos, Argentina, Noviembre 2011.

- M.P. Aguirre, P. Cossutta, J.M. Bracco, M.I. Valla, "Sistema de Acoplamiento a la Red para Microgeneración Fotovoltaica Distribuida". $5^{\circ}$ Congreso Nacional, $4^{\circ}$ Congreso Iberoamericano Hidrógeno y Fuentes Sustentables de Energía HYFUSEN 2013. Córdoba, Argentina, Junio 2013.

- M.P. Aguirre, P. Cossutta, J.M. Bracco, M.I. Valla, "Sincronización con la red de un Convertidor Multinivel Fuente de Corriente con control de balance para generación fotovoltaica". XV RPIC - Reunión de Trabajo en Procesamiento de la Información y Control. Bariloche, Río Negro, Argentina, Septiembre 2013. 


\subsection{Organización y estructura de la Tesis}

En el Capítulo 2 se introducen las estructuras y conceptos básicos de los convertidores electrónicos de potencia multinivel, comenzando por una revisión del funcionamiento y las principales características de las topologías de convertidores fuente de tensión, a fin de obtener las topologías duales de corriente por el principio de dualidad $V-I$ y se analizan los convertidores monofásicos obtenidos. Luego se presentan las topologías fuente de corriente polifásicas, analizándose las diferencias fundamentales con los circuitos monofásicos y los convertidores de tensión. Este análisis lleva a la elección de la topología MCSI Simétrica por sus especiales características que la hacen ideal para aplicaciones industriales y de sistemas eléctricos donde una alta confiabilidad es imprescindible.

En el Capítulo 3 se analiza en profundidad la topología seleccionada, su funcionamiento y control. Se estudia la modulación, la implementación de la lógica Tri-Logic $P W M$ y la máquina secuencial lógica para minimizar el número de conmutaciones por ciclo de las llaves. Se demuestra el funcionamiento tolerante a fallas de la topología y se analiza el funcionamiento de los circuitos de protección agregados. Se presentan las técnicas para el balance de las corrientes internas y se demuestra su funcionamiento mediante simulaciones. También se analiza la conmutación de corriente entre dos llaves consecutivas, identificándose los requerimientos para que se produzca una conmutación "suave" para minimizar las pérdidas en las llaves. Luego se presentan los circuitos y programas utilizados en los desarrollos experimentales, así como los parámetros que intervienen en las simulaciones realizadas para validar las hipótesis planteadas y llegar a la construcción de un prototipo.

En el Capítulo 4 se analizan dos aplicaciones para redes eléctricas de la topología MCSI estudiada, especialmente en aquellas en las que el uso de energía renovables presenta nuevos desafíos. Se presentan resultados de simulación realizados con modelos detallados en Matlab Simulink para verificar los conceptos presentados y analizar la respuesta del convertidor y la aplicación en general.

Finalmente, en el Capítulo 5 se presentan las principales conclusiones obtenidas a lo largo de esta tesis.

En el apéndice se detallan los principales aspectos prácticos de la construcción del prototipo y los modelos de simulación. 


\section{Capítulo 2}

\section{Convertidores Electrónicos de Potencia Multinivel}

\subsection{Introducción}

En este capítulo se presenta un relevamiento de las principales topologías de convertidores multinivel, tanto de tensión (MVSI) como de corriente (MCSI). Si bien los convertidores fuente de corriente han sido estudiados en el pasado y se utilizan en aplicaciones puntuales, distintos factores tecnológicos llevaron a que los convertidores de tensión lideraran las tecnologías durante los últimos años. Es por ello que los MVSI son en estos momentos los convertidores más estudiados y utilizados en la práctica (Hingorani y Gyugyi, 2000; Corasaniti et al., 2008), por lo que se analizan primero en este capítulo. Por otro lado, es posible aplicar el principio de dualidad $V-I$ para obtener topologías de corriente monofásicas equivalentes a las de tensión, a las que puede hacerse extensivo el know-how disponible, simplificando notablemente su análisis. Sin embargo, no todas las topologías de convertidores fuente de corriente multinivel se corresponden con un dual de tensión, presentándose al final del capítulo dos claros ejemplos cuyas ventajas los hacen especialmente atractivos en aplicaciones industriales y de sistemas eléctricos.

Los convertidores multinivel pueden ofrecer algunas ventajas frente a los convertidores tradicionales, ya que se pueden ajustar mejor a los requerimientos especiales de muchas aplicaciones, por ejemplo: en muy alta potencia permiten utilizar llaves de menor tensión y/o corriente, en aplicaciones de baja potencia permiten reducir la EMI generada y la reducción de las pérdidas en las llaves permite aumentar el rendimiento en aplicaciones a baterías o interfaz con energías alternativas. Además, la forma de onda de salida (de corriente en los CSI y de tensión en los VSI) presenta menores distorsiones, es mucho más parecida a una sinusoidal y las componentes de conmutación se encuentran desplazadas hacia altas frecuencias, favoreciendo su filtrado y reduciendo las perturbaciones en las cargas (Grandi 
et al., 2009).

En la Figura 2.1 se comparan las corrientes de salida de un convertidor CSI tradicional y de un MCSI de siete niveles, así como sus componentes en frecuencia. Como puede observarse, la forma de onda de los convertidores tradicionales, con sólo dos niveles activos de corriente, producen una salida con alto contenido de distorsión, en forma de armónicas de la sinusoidal requerida y como componentes de la frecuencia de conmutación. Por otro lado, los convertidores multinivel pueden construir la señal de salida con un mayor número de valores intermedios, con lo cual resulta en una onda que se asemeja más a la sinusoide buscada, con menor contenido de armónicos. Además, el contenido en frecuencia resultante de las conmutaciones se desplaza a una zona mayor del espectro debido al trabajo en conjunto de las distintas llaves, obteniéndose una frecuencia de conmutación equivalente mayor a la de cada llave en forma individual, minimizando sus efectos y facilitando su filtrado.
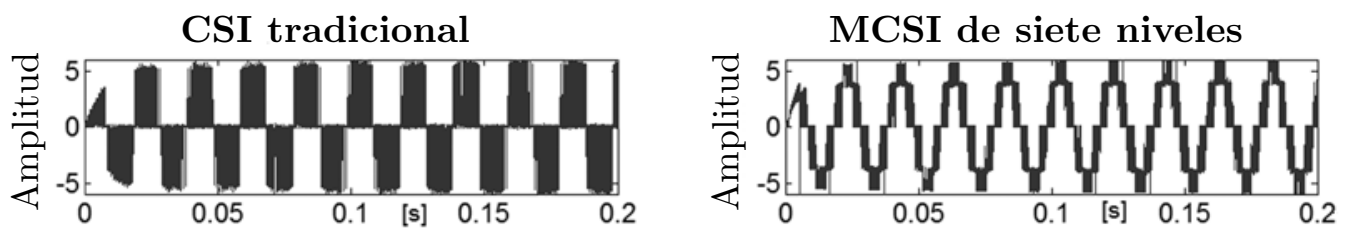

(a) forma de onda de salida
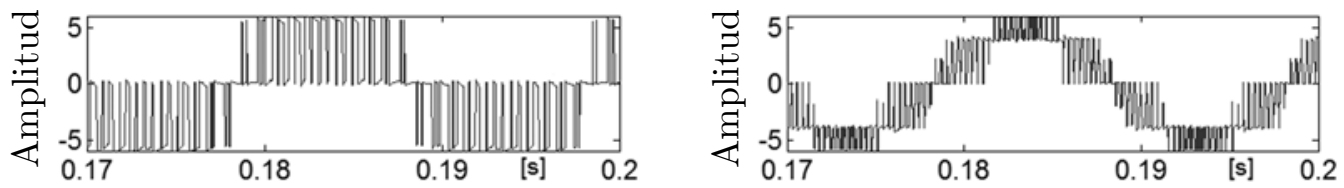

(b) detalle de la forma de onda de salida
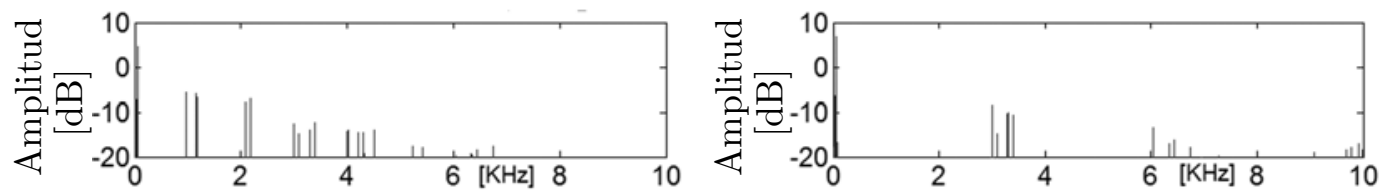

(c) espectro en frecuencia de la salida

Figura 2.1: comparación entre formas de onda: CSI de tres niveles tradicional (izquierda) y MCSI de siete niveles (derecha).

Los convertidores electrónicos tradicionales, especialmente los VSI, son muy utilizados actualmente en aplicaciones de baja y mediana potencia en ambientes industriales de todo tipo, e incluso hasta aplicaciones hogareñas, pero dado que su principio de funcionamiento y aplicaciones pueden ser encontrados en innumerable cantidad de publicaciones no se incluirán en el análisis de este trabajo. En los últimos años, los MVSI han tenido un gran desarrollo en la literatura (Chen y He, 2006; Rodriguez et al., 2007; Krug et al., 2007; Valla et al., 2013), encontrándose cuatro tipos principales: a) enclavamiento por diodos, b) capacitores flotantes, c) configuración en cascada y d) configuraciones híbridas, aunque también se puede encontrar una gran cantidad de topologías con ventajas muy puntuales que se adaptan a ciertas aplicaciones específicas, por ejemplo: Lakshminarayanan et al. (2007), Chen et al. 
(2008) y Flores et al. (2009). Por otro lado los MCSI no han sido estudiados en profundidad y pocos esfuerzos se han realizado para su clasificación, siendo el trabajo más completo el de Bai y Zhang (2008a), por lo que resulta importante realizar un análisis comparativo previo de las topologías existentes a fin de poder seleccionar una de ellas para su estudio exhaustivo. De esta manera, se presentan en este capítulo una revisión de las principales topologías de MVSI y se presentan tres topologías de MCSI para poder comparar sus estructuras circuitales, ventajas y desventajas.

\subsection{Convertidores Multinivel Fuente de Tensión}

Los MVSI han sido estudiados ampliamente en la literatura, presentando una alternativa a los VSI de dos niveles en aquellas aplicaciones de alta potencia, alta tensión o corriente y donde el ripple debe ser reducido, entre otras publicaciones se pueden citar las de Lai y Peng (1996); Rodriguez et al. (2002, 2007) y Krug et al. (2007). La estructura trifásica típica de estos convertidores consta de tres ramas, para generar cada una de las tensiones de fase, con la posibilidad de agregar una cuarta rama para manejar la corriente de neutro y así poder controlar sistemas con fuertes desbalances. Para conectar un MVSI a un sistema eléctrico deben utilizarse inductores de acoplamiento, ya que siendo fuentes de tensión no pueden conectarse a otra fuente de tensión sin violar las leyes de Kirchoff. Los inductores de acoplamiento permiten inyectar o tomar del sistema una corriente $i_{L}$ que dependerá de la diferencia de amplitud y fase entre la tensión generada por el convertidor y la presente en el sistema (Mohan et al., 2005). Se han desarrollado distintas topologías, y cada una de las cuales tiene ventajas y desventajas con respecto a la utilización de las llaves, el uso de capacitores y simetrías, entre otras.

\subsubsection{Configuración en Cascada}

La configuración en cascada es una interesante opción para el desarrollo de aplicaciones de alta potencia ya que presenta una construcción modular que facilita su diseño, operación y mantenimiento. Su estructura, Figura 2.2(a), se basa en la conexión en serie de módulos VSI tipo Puente Completo o Puente-H (Dixon y Moran, 2002; Rodriguez et al., 2003; Ertl et al., 2002), los que tienen la ventaja de haber sido ampliamente utilizados por mucho tiempo en ambientes industriales por lo que su construcción y operación es muy conocida (Jana et al., 2006; Malinowski et al., 2010; de Alvarenga y Pomilio, 2011). La principal desventaja de esta topología es que requiere de fuentes de alimentación independiente para cada módulo, lo que implica seis fuentes aisladas para un convertidor trifásico de cinco niveles, fuerza el uso de transformadores para su alimentación, complica su utilización con energías alternativas y hace imposible su utilización en topologías Back-to-Back (Sección 2.2.3) sin recurrir a complejos esquemas con convertidores CC/CC aislados (Ertl et al., 2002). 


\subsubsection{Capacitores Flotantes}

La estructura básica de esta topología se muestra en la Figura 2.2(b), donde se puede observar que en el punto medio de cada par de llaves se conecta un capacitor que enclava la tensión de ese punto y por lo tanto la tensión aplicada a la carga para cada combinación de encendido de las llaves. La estructura de un convertidor trifásico se construye con tres ramas, donde cada una de ellas generará una de las tensiones de fase en la carga, por lo que para un convertidor de cinco niveles se requiere un total de nueve capacitores aislados entre si. La baja confiabilidad de los capacitores de alta tensión y alta potencia es una de las principales desventajas de esta topología, además los capacitores internos no pueden conectarse directamente en configuración Back-to-Back, aunque sí se puede realizar esta configuración a través del bus de continua principal. Por otro lado, se puede conseguir un buen balance de tensión en los capacitores mediante el uso de una modulación adecuada, como por ejemplo la Modulación por Ancho de Pulso con Portadoras Desfasadas (PSC-SPWM).

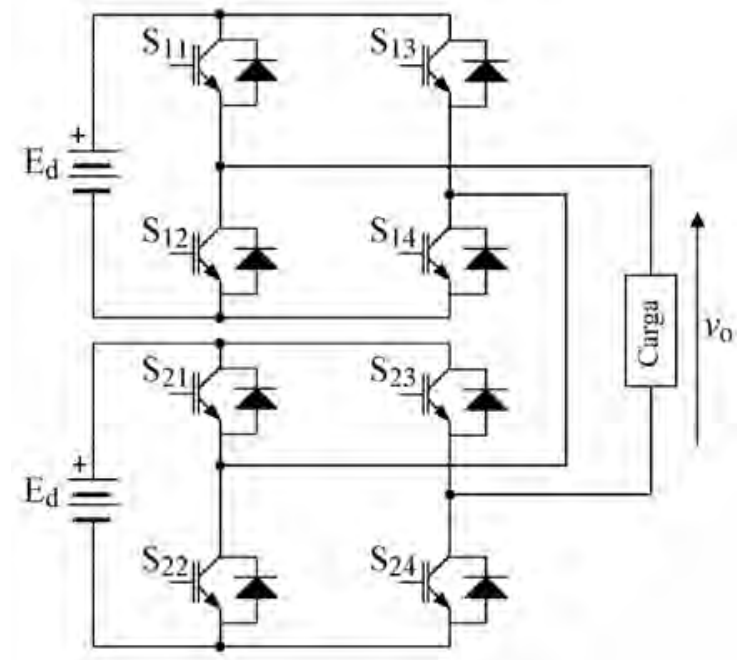

(a) puente $\mathrm{H}$ en cascada.

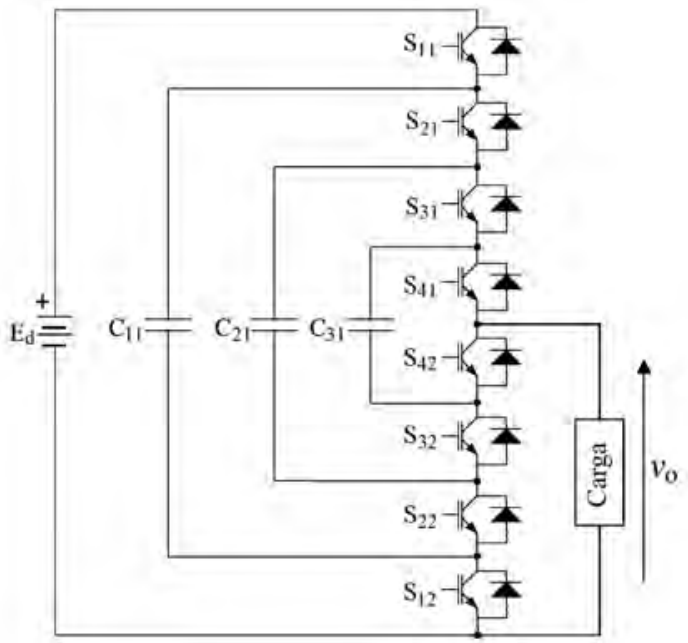

(b) capacitores flotantes.

Figura 2.2: MVSI monofásicos de cinco niveles.

\subsubsection{Enclavamiento por diodos}

Esta topología se denomina Diode Clamp Multilevel Converter (DCMC), pero su versión de tres niveles se conoce también como Neutral Point Clamped (NPC) (Nabae et al., 1981; Rodriguez et al., 2010; Valla et al., 2013), dado que el enclavamiento de tensión de los diodos se produce al punto medio del divisor capacitivo de entrada. La estructura básica consiste en el agregado de diodos a una rama de llaves en serie como se muestra en la Figura 2.3(a). Los diodos enclavan la tensión en el punto medio entre cada par de llaves al valor presente en uno de los capacitores del bus de continua. El banco de capacitores en serie divide la tensión del bus de continua $E_{d}$ en partes iguales, lo que permite generar los distintos niveles de tensión a la salida. 


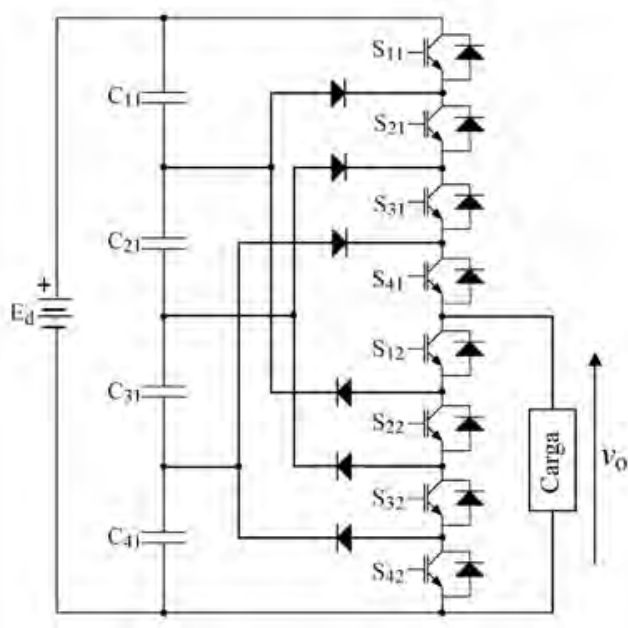

(a) original.

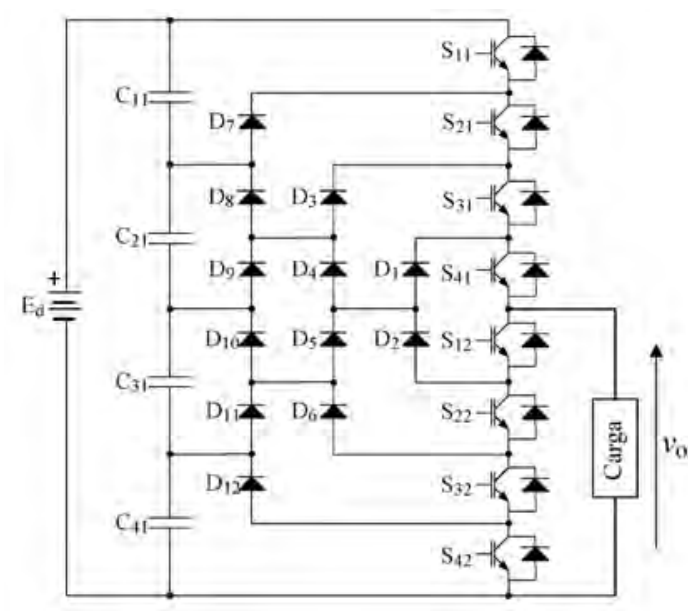

(b) modificado.

Figura 2.3: MVSI de enclavamiento por diodos monofásico de cinco niveles.

Como sucede en la mayoría de las topologías de MVSI, las llaves de los DCMC deben soportar sólo una fracción de la tensión del bus de continua, pero uno de los problemas principales de la topología, cuyo convertidor monofásico se muestra en la Figura 2.3(a), es que los diodos deben soportar distintas fracciones de la tensión del bus de continua $\left(E_{d}\right)$, por lo que en altas tensiones se deben utilizar complejas configuraciones de diodos en serie en los cuales es muy difícil ecualizar las tensiones en inversa y los tiempos de conmutación. Debido a este problema se han desarrollado topologías alternativas, como la mostrada en la Figura 2.3(b), donde se utilizan más diodos pero que deben soportar todos la misma fracción de la tensión $E_{d}$, al igual que las llaves. Esto simplifica el diseño de las estrategias de modulación y disparo de las llaves ya que los tiempos de conmutación de las llaves y los diodos dependen de la tensión que deban soportar y de esta manera se tienen tiempos similares en todos los dispositivos.

En la Figura 2.4 se muestra la configuración trifásica de un DCMC de cinco niveles. Cada rama del convertidor genera la tensión de una fase y las tres ramas comparten el bus de continua y los capacitores divisores de tensión, esto facilita el desarrollo de técnicas de modulación vectorial que mantengan el balance de tensiones en esos capacitores (Rodriguez et al., 2007; Krug et al., 2007; Verne y Valla, 2010a,b). El propio funcionamiento del convertidor, sumado a las asimetrías en los valores de los componentes y en los tiempos de conmutación de las llaves conllevan un inevitable desbalance por lo que es indispensable contar con una estrategia de control para asegurar la correcta distribución de tensiones. Esta topología no presenta redundancia de alternativas de conmutación para realizar el mismo nivel de tensión en una fase y ello requiere el desarrollo de complejos algoritmos de modulación para conseguir objetivos de control específicos (Marchesoni et al., 1998). El balance de tensiones en los capacitores del bus de continua es una de las principales dificultades que se deben resolver para su utilización en aplicaciones industriales, ya que existe una relación de compromiso entre el factor de potencia en la carga y el máximo índice de modulación en amplitud $\left(\mathrm{m}_{a}\right)$ 


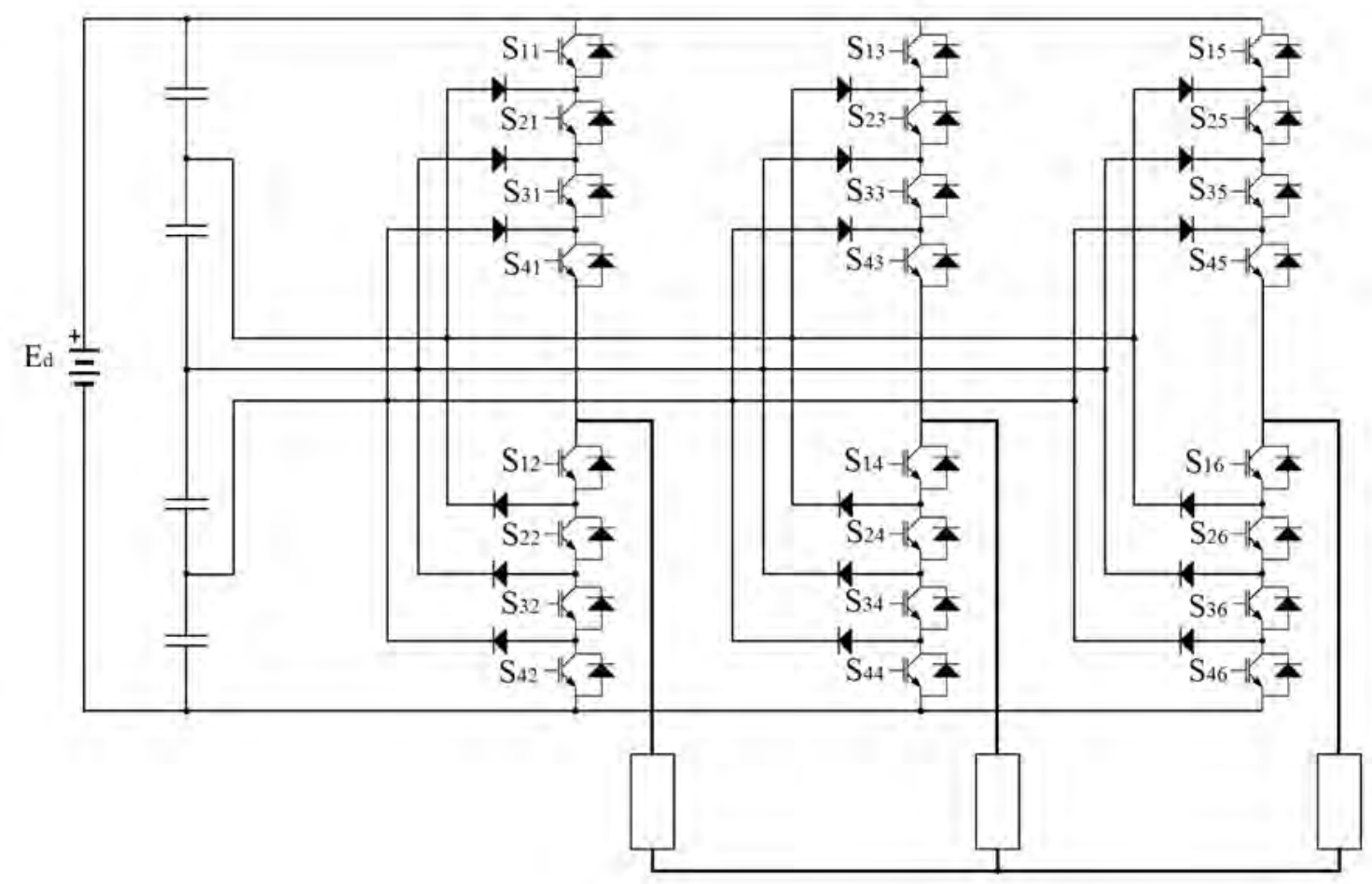

Figura 2.4: diagrama completo de un MVSI de enclavamiento por diodos trifásico de cinco niveles.

que se puede generar con el convertidor (Marchesoni y Tenca, 2002; Ceballos et al., 2011), pero el tema está ampliamente tratado y se presentan diversas soluciones en la literatura, como por ejemplo Verne y Valla (2010a) que en su trabajo proponen el uso de una función de costo para evaluar la mejor alternativa de conmutación en cada instante de tiempo a fin de minimizar la frecuencia de conmutación y mantener el balance de tensiones en los capacitores.

La gran cantidad de dispositivos de conmutación de los DCMC implica mayores pérdidas y menor confiabilidad, por lo que en ese aspecto la topología de capacitores flotantes se presenta como una alternativa con mayor rendimiento. Sin embargo, debe considerarse que la tecnología de capacitores flotantes requiere capacitores de alta confiabilidad, con tensiones de trabajo distintas y que deben mantener el mismo valor de capacidad a lo largo de toda su vida útil, lo cual configura una importante restricción de diseño. Es por ello que la elección de la topología de convertidores adecuada para una cierta aplicación implica un complejo estudio donde debe analizarse una gran cantidad de variables y establecer compromisos entre los distintos parámetros involucrados.

\section{Conexión Back-to-Back de convertidores DCMC}

La topología DCMC permite la conexión Back-to-Back en la que dos convertidores se unen compartiendo los mismos capacitores del bus de continua, como se muestra en la Figura 2.5. Esta configuración permite la conversión entre dos sistemas de corriente alterna de distinta 
tensión, fase o frecuencia $(\mathrm{CA} / \mathrm{CA})$, pasando por una etapa intermedia de corriente continua (CC). La utilización de un solo bus de continua permite la optimización de los algoritmos de balance de tensión ya que ambos convertidores pueden operar sobre la corriente de los capacitores para mantener balanceadas las tensiones para todos los puntos de trabajo de la carga. Esto elimina la relación limitante entre el factor de potencia en la carga y el $m_{a}$, permitiendo que los convertidores puedan entregar tanto potencia activa como reactiva (Valla et al., 2013).

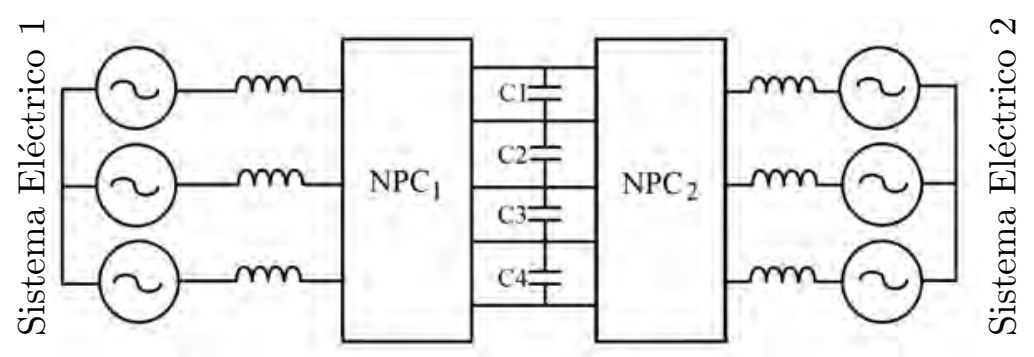

Figura 2.5: conexión de dos DCMC en configuración "espalda con espalda" (back-to-back).

\subsubsection{Configuración Híbrida}

Se han desarrollado configuraciones híbridas que interconectan celdas básicas de distintas topologías a fin aprovechar las ventajas y compensar sus desventajas (Chen y He, 2006; Rech y Pinheiro, 2007). En la Figura 2.6 se muestra una configuración de este tipo que ha sido ampliamente analizada por Gonzalez et al. (2010). Esta topología tiene la ventaja de requerir diodos de enclavamiento de baja tensión y que los capacitores de $E_{d} / 2$ pueden compartirse entre las tres ramas y además conectarse en configuración Back-to-Back, mientras que los capacitores flotantes $\left(E_{d} / 4\right)$ deben estar aislados.

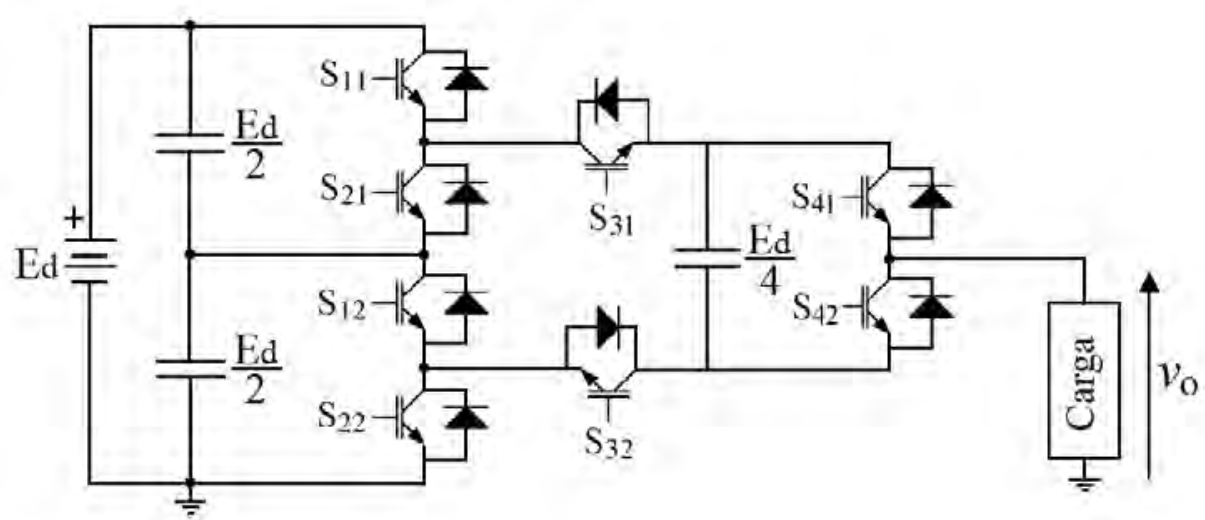

Figura 2.6: MVSI de configuración híbrida de cinco niveles. 


\subsection{Convertidores Multinivel Fuente de Corriente monofási- $\cos$}

Los CSI de tres niveles han sido utilizados en el pasado en aplicaciones de alta corriente, como el control de grandes motores y sistemas eléctricos (Hombu et al., 1985; Nonaka y Neba, 1987; Zargari et al., 1997; Hingorani y Gyugyi, 2000), pero como la conmutación de corriente en cargas inductivas produce sobretensiones e incrementa el estrés en las llaves, y además se desarrollaron llaves electrónicas con posibilidad de apagado controlado, lentamente los VSI fueron superándolos en casi todas las aplicaciones. Al igual que sucede para los convertidores de tensión, los MCSI tienen algunas ventajas sobre los CSI de tres niveles, como por ejemplo: los MCSI tienen más grados de libertad para generar el mismo nivel de corriente de salida, en general tantos como inductores fuente de corriente haya en el circuito (Xiong et al., 2004a), alimentan en todo momento a las tres fases de la carga y la someten a escalones o saltos de corriente mucho menores, posibilitando su uso en aplicaciones altamente inductivas sin necesidad de agregar grandes capacidades en paralelo para compensar las sobretensiones de conmutación. La principal desventaja radica en que los inductores suelen ser mucho más voluminosos y pesados que los capacitores utilizados en los MVSI, sin embargo su vida útil es mucho mayor y requieren menor mantenimiento. Para ciertas aplicaciones, en las que se requiere bajo ripple de corriente, o media tensión y alta corriente, es posible utilizar topologías MCSI en las que el número de niveles y el uso de llaves en paralelo presentan grandes ventajas. Se han desarrollado variedad de versiones (Antunes et al., 1999; Bai y Zhang, 2008a; McGrath y Holmes, 2008a; Bai et al., 2008; Suroso y Noguchi, 2010; Vazquez et al., 2010; Liang et al., 2013), algunas de las cuales tienen posibilidades de uso en ambientes industriales y por ello se presentan en esta sección.

\subsubsection{Obtención de topologías por el principio de dualidad V-I}

El principio de dualidad Tensión-Corriente (Bai y Zhang, 2008a) es una herramienta poderosa para poder transformar una topología conocida en su dual, ya que en la mayoría de los casos la topología dual tendrá las mismas características y por lo tanto se puede utilizar el conocimiento adquirido para la topología original. Sin embargo, el principio de dualidad sólo puede ser aplicado a circuitos del tipo planar, que son aquellos que se pueden dibujar sin cables que se crucen sin tener conexión eléctrica y por ello se utiliza principalmente para obtener celdas básicas, generalmente monofásicas, y a partir de ellas se construye la topología MCSI real que puede no ser el dual exacto de un MVSI.

El primer paso para obtener una topología dual es convertir la funcionalidad de las llaves, para luego intercambiar, mediante los equivalentes de Thévenin y Norton, mallas por nodos y fuentes de tensión por fuentes de corriente.

En los MVSI las llaves deben tener capacidad de bloqueo unidireccional de tensión y conducción bidireccional de corriente, en los MCSI se requiere entonces conducción unidireccional de corriente y bloqueo bidireccional de tensión. Las llaves electrónicas de potencia en la actualidad (IGBT, IGCT, ETO, etc.) no permiten el bloqueo bidireccional 
de tensión, ya que su estructura interna, tanto de ánodo transparente como de ánodo en cortocircuito, no soporta la misma tensión en inversa que en directa, es por ello que aprovechando que la corriente circula en un solo sentido se puede agregar un diodo en serie que permita el bloqueo de tensión inversa como se muestra en la Figura 2.7.

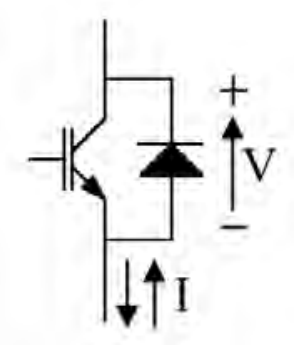

(a) Llave para MVSI

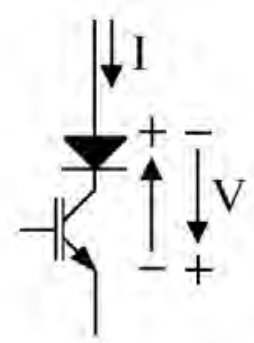

(b) Llave para MCSI

Figura 2.7: dualidad Tensión-Corriente aplicada a las llaves electrónicas de potencia.

Se pueden obtener tres convertidores MCSI monofásicos a partir de los convertidores MVSI conocidos:

\subsubsection{Cascada}

Esta topología tiene como principales ventajas su construcción modular y la ausencia de inductores divisores, mientras que las principales desventajas son que requiere fuentes aisladas, al igual que su dual de tensión, y mayor cantidad de llaves para el mismo número de niveles a la salida que otras topologías. En la Figura 2.8 se muestra el diagrama esquemático para un convertidor de cinco niveles, donde cada módulo puede generar dos niveles en la corriente $\left( \pm \mathrm{I}_{d}\right)$, con lo que puede obtenerse en la carga $+2 \mathrm{I}_{d},+\mathrm{I}_{d}, 0,-\mathrm{I}_{d} \mathrm{y}-2 \mathrm{I}_{d}$, y por lo tanto puede utilizarse como convertidor monofásico ya que genera corrientes alternas.

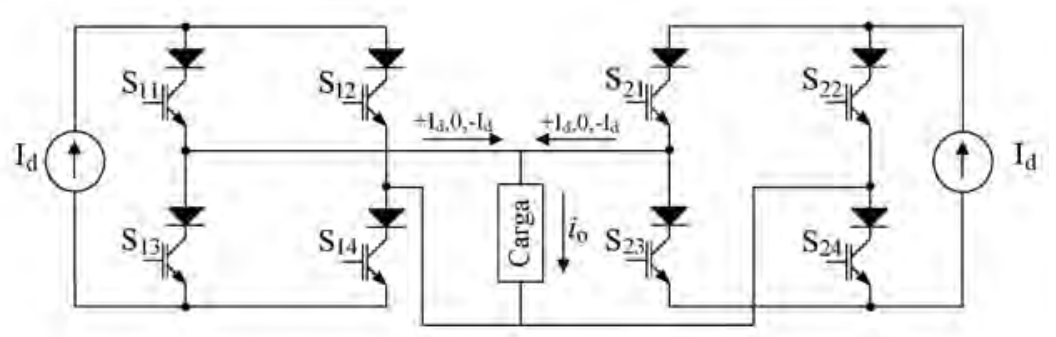

Figura 2.8: MCSI cascada monofásico.

\subsubsection{MCSI Asimétrico}

Esta topología se puede obtener mediante la dualidad $V-I$ a partir del circuito de capacitores volantes de la Figura 2.2(b) (Bai y Zhang, 2008a). Como se muestra en la 
Figura 2.9, se necesitan dos llaves para generar cada nivel de corriente de salida. Cada inductor debe conducir una fracción distinta de la corriente principal, por lo que su diseño y mantenimiento resultan más complicados. Su funcionamiento permite redundancia en las combinaciones de llaves necesarias para obtener un cierto nivel de corriente en la salida, cada llave superior que se enciende resta en la carga $1 / 4 I_{d}$, mientras que la llave complementaria permanece cerrada. En la Figura 2.9(b), la corriente de salida es $3 / 4 I_{d}$, forzada por los tres inductores $\mathrm{L}_{1}$ a $\mathrm{L}_{3}$ ya que las llaves $\mathrm{S}_{22}, \mathrm{~S}_{32}$ y $\mathrm{S}_{42}$ están encendidas. El resto de la corriente principal, $1 / 4 I_{d}$, circula por la llave $\mathrm{S}_{11}$. Otro ejemplo de combinaciones de conmutación se muestra en la Figura 2.9(c), donde una corriente de salida de $1 / 2 I_{d}$ se obtiene al encender dos llaves superiores, en este caso $\mathrm{S}_{31}$ y $\mathrm{S}_{41}$, mientras que la corriente retorna a la fuente principal a través de las llaves $\mathrm{S}_{12}$ y $\mathrm{S}_{22}$. La versión monofásica de esta topología sólo puede generar corrientes en la carga en un sentido, por lo que su utilización se restringe sólo a aquellas aplicaciones en las que la carga requiere un nivel de corriente continua variable (Suroso y Noguchi, 2010).

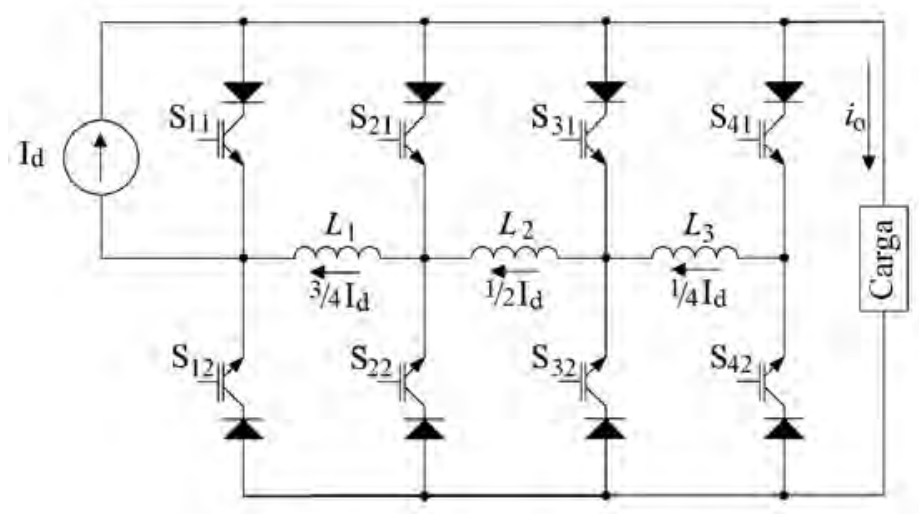

(a)

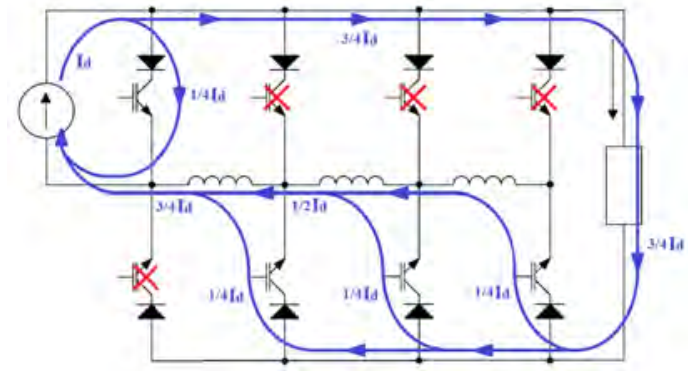

(b) $i_{o}=3 / 4 I_{d}$

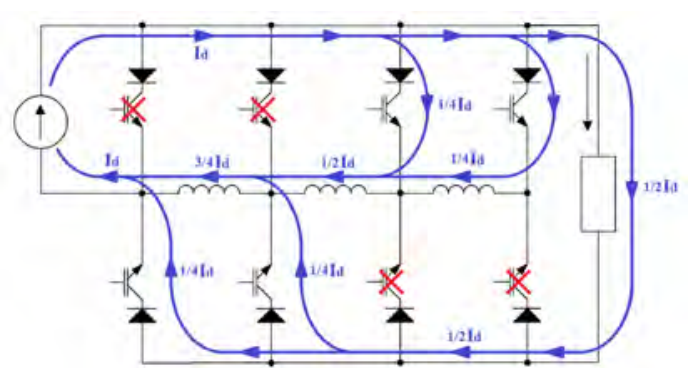

(c) $i_{o}=1 / 2 I_{d}$

Figura 2.9: asimétrico monofásico de cinco niveles, circuito básico y dos ejemplos de conmutación.

\subsubsection{MCSI Simétrico}

Mediante la aplicación del principio de dualidad V-I es posible partir de la topología DCMC (Bai y Zhang, 2008a), utilizando su versión modificada de la Figura 2.3(b), para 
obtener la topología de la Figura 2.10, en la que se utilizan dos llaves y un inductor para generar cada nivel en la corriente de salida. El convertidor resultante puede generar cinco niveles en la corriente de la carga (contando el nivel cero). Si bien utiliza un inductor más que la celda anterior, su ventaja principal es que todos los inductores y todas las llaves tienen que conducir la misma fracción de la corriente principal, lo que simplifica su diseño, operación y mantenimiento. Al igual que el anterior, este convertidor monofásico sólo puede generar corrientes en un sentido, por lo que no puede utilizarse en aplicaciones donde deba evitarse una componente de corriente continua en la carga.

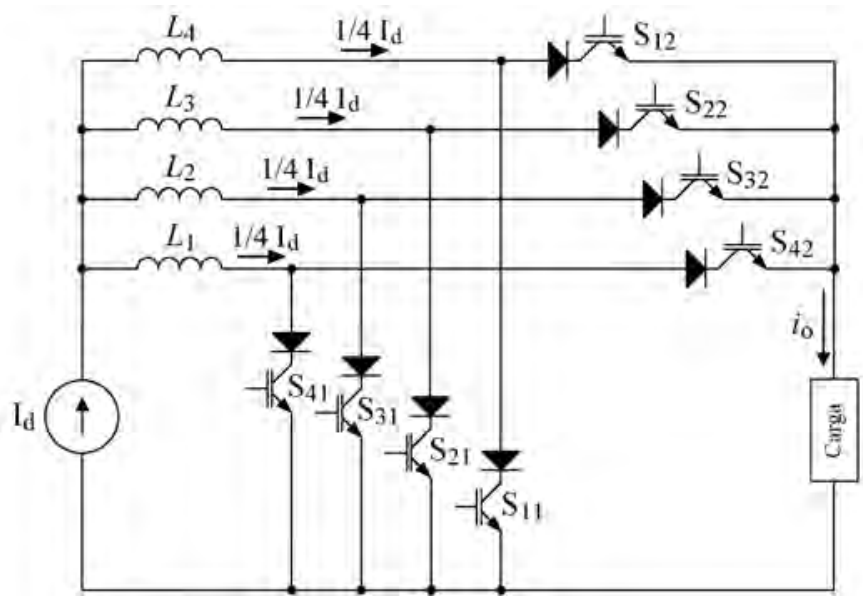

Figura 2.10: MCSI Simétrico monofásico de cinco niveles.

\subsection{Convertidores Multinivel Fuente de Corriente polifásicos}

En los MVSI las estructuras se forman a nivel de la pierna que genera la tensión de una fase, completándose el convertidor con tantas piernas idénticas como fases requiera la aplicación, tres en sistema balanceados y cuatro en sistemas con fuertes desbalances donde es necesario un control directo de la tensión del neutro. Todas las piernas comparten el bus de continua y la tensión de cada una se modula en forma independiente para controlar la corriente que requiere la aplicación, sobre todo en aplicaciones de sistemas eléctricos donde la acción del convertidor se realiza a través de la corriente inyectada. Lo mismo ocurre en los MCSI en Cascada, donde se puede armar una pierna con tantos puentes $H$ como niveles $(2 n+1)$ se requiera a la salida y luego construir el convertidor con tantas piernas como fases requiera el sistema.

Pero por el contrario, en el resto de las topologías MCSI estudiadas, la corriente en la carga está definida por cada módulo, en las tres fases, en forma simultánea. De esta manera, el convertidor completo queda definido con al menos un módulo y el agregado de más módulos sólo aumenta la cantidad de niveles en la corriente de salida y por lo tanto la cantidad de módulos no está relacionada con el número de fases de la carga. Esto conlleva a un análisis de todo el sistema como un conjunto sin la posibilidad de estudiar una pierna o fase por separado y luego extrapolar el análisis al convertidor. Pero tiene la ventaja de permitir estrategias 
tolerantes a fallas, ya que la corriente en todas las fases de la carga puede ser mantenida por al menos un módulo mientras se repara o cambia un módulo dañado.

\subsubsection{MCSI en Cascada trifásicos}

En la Figura 2.11 se muestra la conexión trifásica de un convertidor MCSI en cascada. Cada módulo de cuatro llaves genera dos niveles activos y cero, en la corriente de una fase, por lo que se requieren veinticuatro llaves para producir los cinco niveles requeridos. Esta topología utiliza más llaves para producir cierta cantidad de niveles en la corriente de salida que las otras presentadas en este capítulo, pero su construcción modular permite un rápido diseño y una mejor operación y mantenimiento. Se puede observar que se requieren seis fuentes de corriente independientes para su funcionamiento lo cual complica su utilización, requiriendo generalmente transformadores con una gran cantidad de bobinados. Podría simplificarse su utilización en el caso de contar con fuentes independientes, como en el caso de utilizar celdas solares, pero debe velarse por la simetría entre todas para evitar distorsiones y deformaciones en la salida trifásica. Su principal ventaja radica en su estructura modular y simétrica, existiendo numerosa información y ejemplos en la bibliografia (Rodriguez et al., 2003; Jana et al., 2006; Samuel et al., 2011; de Alvarenga y Pomilio, 2011; Verma et al., 2011; Al-Hanai et al., 2011).

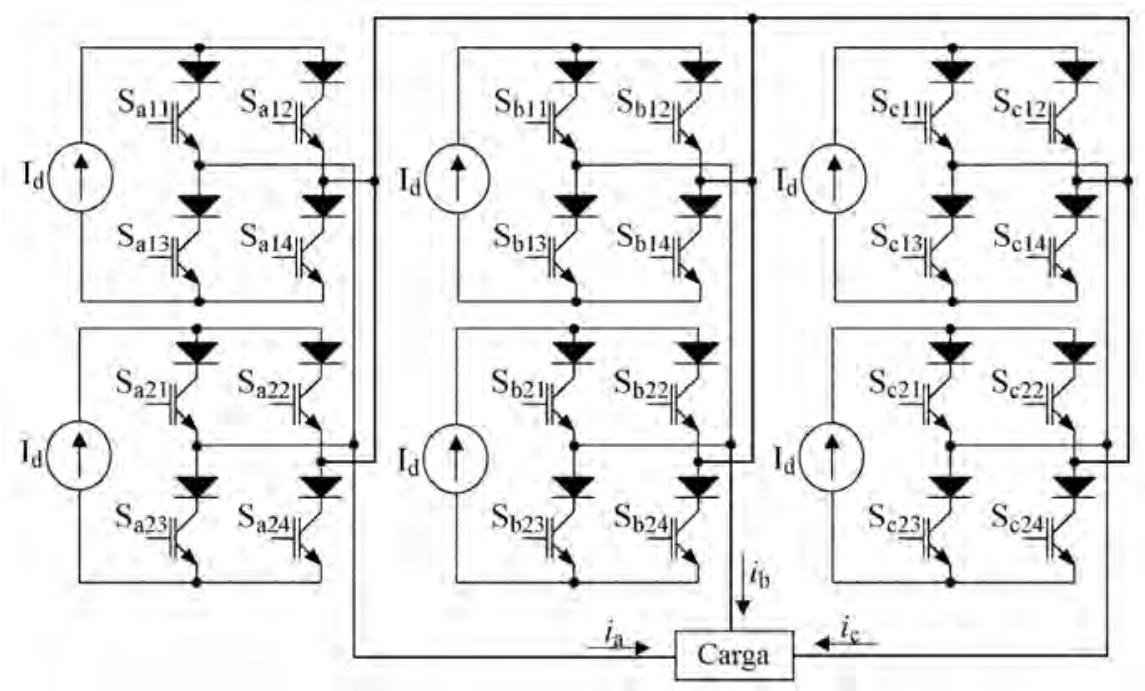

Figura 2.11: convertidor MCSI en cascada trifásico de cinco niveles.

\subsubsection{Multi Rating Inductor MCSI}

Al obtener topologías MCSI mediante el principio de dualidad, es de esperarse que el circuito resultante genere la corriente en cada fase mediante una rama independiente del convertidor, lo que no ocurre en la versión trifásica del convertidor MCSI Asimétrico, denominado en la bibliografía Multi Rating Inductor MCSI (Bai y Zhang, 2008a; Bai et al., 2007), cuyo diagrama esquemático se muestra en la Figura 2.12. Esto es así porque esta 
topología no es del tipo planar dado que cada módulo genera corrientes que se suman para construir la corriente de las tres fases de la carga en simultáneo. Es por ello que esta topología no es el dual de ninguna de las topologías de tensión analizadas en la literatura.

En estos convertidores, cada inductor regula la fracción de corriente de la fuente principal $\mathrm{I}_{d}$ que circula por él, fijando la corriente por el grupo de seis llaves asociado y por lo tanto el aporte de ese grupo de llaves a la carga. De esta manera los inductores $\mathrm{L}_{11}$ y $\mathrm{L}_{12}$ conducirán $3 / 4$ de la corriente principal $\mathrm{I}_{d}, \mathrm{~L}_{21}$ y $\mathrm{L}_{22}$ la mitad de $\mathrm{I}_{d}$ y $\mathrm{L}_{31}$ y $\mathrm{L}_{32}$ sólo $1 / 4$ de $\mathrm{I}_{d}$. Como consecuencia, cada grupo de seis llaves conducirá la diferencia entre la corriente proveniente del inductor anterior y la que drena el inductor siguiente, haciendo que todas ellas conduzcan la misma fracción de la corriente principal, en este caso $1 / 4 \mathrm{I}_{d}$. Esto permite la utilización de llaves de menor corriente, ecualizando las pérdidas y tiempos de conmutación al conducir todas la misma corriente. En el Cuadro 2.1 se resumen los valores de corriente para un convertidor de nueve niveles como el de la Figura 2.12. Para la generación de las señales de disparo de las llaves pueden utilizarse modulaciones de portadoras desfasadas en nivel, tales como PD, POD, APOD las que se describen en profundidad en (Holmes y Lipo, 2003), o la PSC-SPWM (Bai et al., 2007). La principal desventaja de esta topología es que no puede construirse en forma modular, complicando su diseño, operación, mantenimiento y desarrollo de convertidores tolerantes a fallas para aplicaciones industriales.

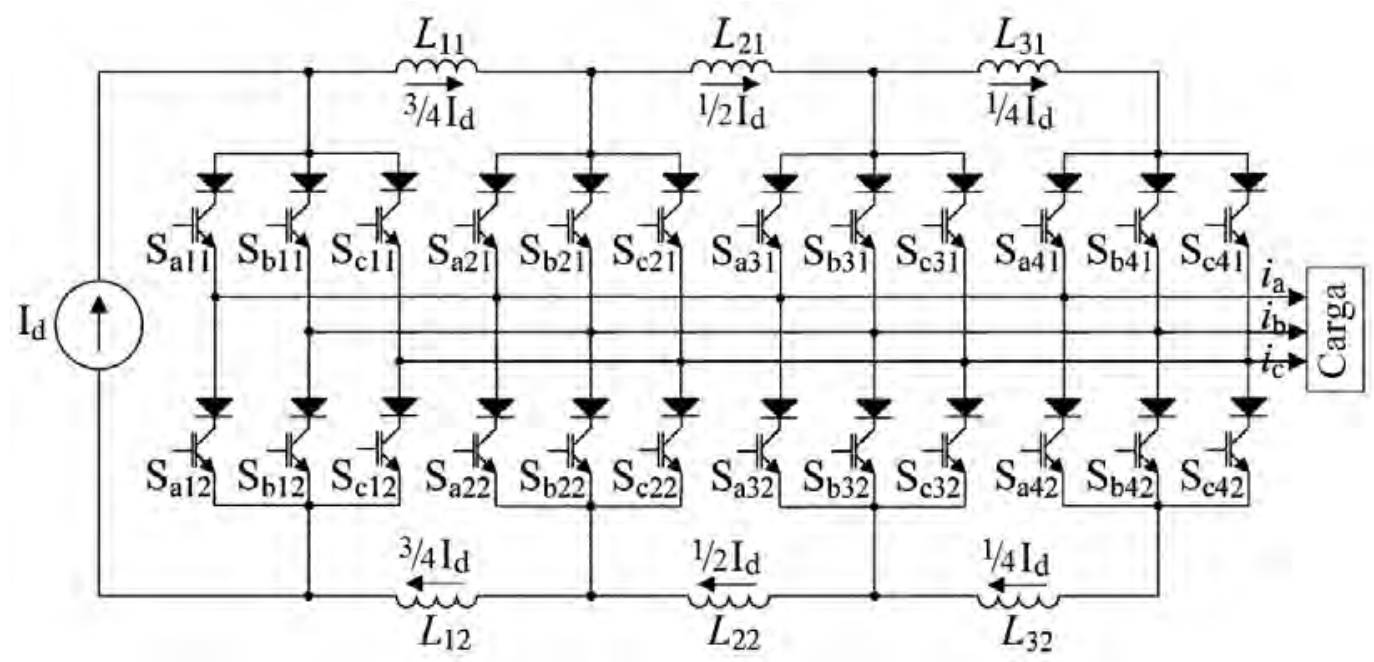

Figura 2.12: convertidor MCSI Asimétrico trifásico de nueve niveles.

Cuadro 2.1: resumen de las corrientes involucradas en el circuito de la Figura 2.12.

\begin{tabular}{cccccccccc}
\hline $\mathrm{L}_{11}$ & $\mathrm{~L}_{12}$ & $\mathrm{~L}_{21}$ & $\mathrm{~L}_{22}$ & $\mathrm{~L}_{31}$ & $\mathrm{~L}_{32}$ & $\mathrm{~S}_{a} 11$ & $\mathrm{~S}_{a} 21$ & $\mathrm{~S}_{a} 31$ & $\mathrm{~S}_{a} 41$ \\
$3 / 4 \mathrm{I}_{d}$ & $3 / 4 \mathrm{I}_{d}$ & $1 / 2 \mathrm{I}_{d}$ & $1 / 2 \mathrm{I}_{d}$ & $1 / 4 \mathrm{I}_{d}$ & $1 / 4 \mathrm{I}_{d}$ & $1 / 4 \mathrm{I}_{d}$ & $1 / 4 \mathrm{I}_{d}$ & $1 / 4 \mathrm{I}_{d}$ & $1 / 4 \mathrm{I}_{d}$ \\
\hline
\end{tabular}




\subsubsection{Single Rating Inductor MCSI}

Esta topología, denominada también MCSI Simétrico, cuyo circuito para un convertidor de nueve niveles se muestra en la Figura 2.13, consta de múltiples módulos CSI que comparten la corriente de entrada $\mathrm{I}_{d}$ y entregan una fracción de esta corriente a la carga (Bai y Zhang, 2008a; Suroso y Noguchi, 2010). Al igual que los Multi Rating Inductor no son circuitos del tipo planar, por lo que no puede extrapolarse su análisis por dualidad a partir de ningún MVSI y por ello es preciso analizarlos en forma independiente. El funcionamiento de estos convertidores es básicamente distinto al de un MVSI, ya que cada módulo genera corriente en las tres fases de la carga y por lo tanto no puede estudiarse como la suma de circuitos monofásicos. Esto implica que el ripple de tensión en uno cualquiera de los inductores divisores de un módulo estará dado por las características de la carga y por el accionar de las llaves de los otros módulos del convertidor, constituyendo así un circuito totalmente acoplado que debe analizarse en conjunto. En estos convertidores, cada módulo consiste en seis llaves y dos inductores, y la principal ventaja de esta topología es que cada inductor y cada llave conducen la misma fracción de $\mathrm{I}_{d}$, por lo que todos los módulos son idénticos y tienen idéntico régimen de trabajo.

La estructura modular permite simplificar el diseño, la operación y el mantenimiento de los convertidores. Además facilita el desarrollo de metodologías de funcionamiento tolerante a fallas. Estas especiales características los hacen ideales para aplicaciones en sistemas eléctricos e industriales y por esta razón se eligen para su estudio en detalle en los capítulos siguientes.

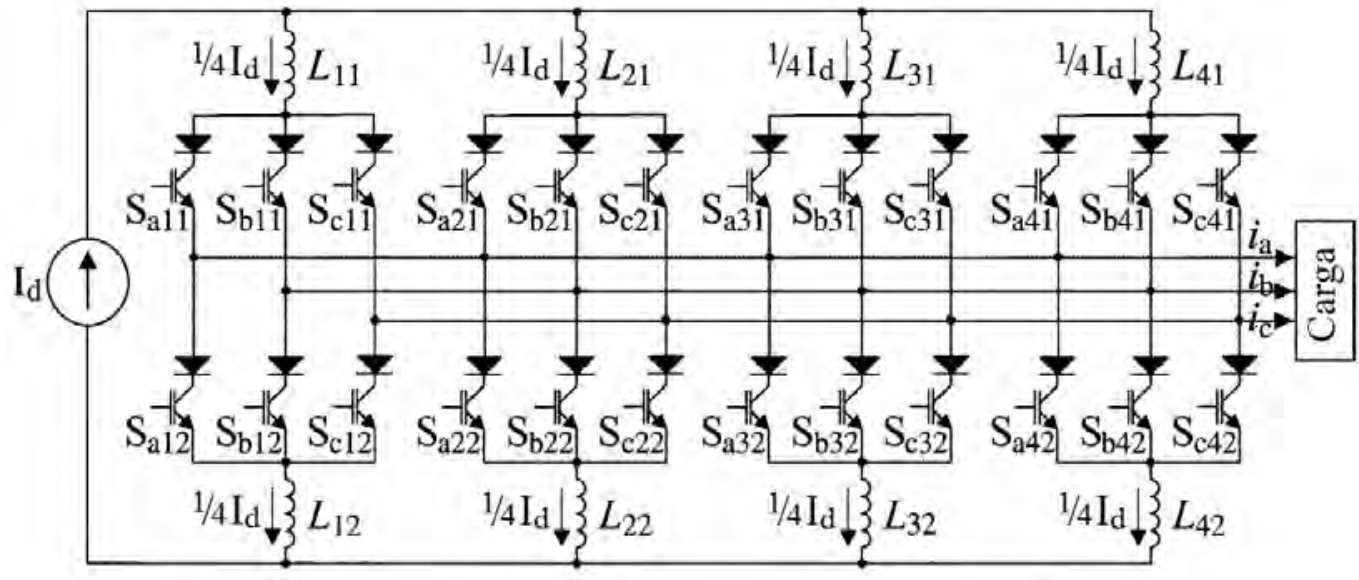

Figura 2.13: convertidor MCSI Simétrico trifásico de nueve niveles. 


\section{Capítulo 3}

\section{MCSI Simétrico Tolerante a Fallas}

\subsection{Introducción}

En este capítulo se propone el análisis detallado de la topología MCSI Simétrico, seleccionada debido a sus especiales características de modularidad y funcionamiento, que la hacen ideal para aplicaciones de alta potencia. Se estudia la aplicación de la Modulación de Ancho de Pulso con Desplazamiento de Fase de Portadoras (PSC-SPWM) para la generación de las señales de disparo de las llaves y sus ventajas en cuanto a capacidad de cálculo requerida y la generación de un inherente balance de las corrientes internas (Aguirre et al., 2009, 2010b).

Se demuestra que es posible minimizar la cantidad de conmutaciones de las llaves por ciclo, maximizando el rendimiento del convertidor y permitiendo elevar la frecuencia de conmutación para obtener una excelente respuesta dinámica. Esto se logra mediante la correcta selección de los estados cero en cada momento, gracias al diseño de una exclusiva máquina secuencial lógica programable en una FPGA (Aguirre et al., 2010a; Tettamanti et al., 2011).

Se presenta además el estudio de la conmutación de las llaves a fin de diseñar correctamente el hardware para obtener una conmutación "suave" que facilita la libre transferencia de corriente entre dos llaves que conmutan en forma consecutiva, minimizando las pérdidas por conmutación. Esto permite además obtener una mayor fidelidad de los resultados de simulación y escalar los resultados obtenidos a potencias mayores (Aguirre et al., 2013a).

Se presenta y analiza una estrategia de tolerancia a fallas que, mediante la conmutación de un módulo de respaldo, permite garantizar el normal funcionamiento del convertidor frente a una falla en alguno de sus componentes. Esto permite también el ciclado de los módulos activos para tareas de mantenimiento preventivo, funcionalidad indispensable en equipos industriales o de acondicionamiento de redes eléctricas (Aguirre et al., 2009, 2010b).

Debido a que el balance de las corrientes internas producido por la modulación utilizada es 
débil y presenta error en régimen permanente, se han desarrollado dos métodos para controlar el valor medio de las corrientes internas del convertidor que, utilizando una mínima potencia de cálculo, permiten garantizar el balance interno frente a perturbaciones, dispersión en los valores de los componentes y diferencias en los tiempos de conmutación de las llaves (Aguirre et al., 2012a, 2013c).

El número de módulos, y por lo tanto de niveles de corriente en la salida, depende de la relación óptima entre la aplicación, costo y prestaciones del convertidor, interviniendo innumerable cantidad de parámetros como por ejemplo: frecuencia de ripple deseada a la salida, frecuencia de conmutación de cada llave, distorsión máxima aceptable en la corriente de salida, potencia total del convertidor y de cada llave, eficiencia requerida, potencia, confiabilidad y tolerancia a fallas y velocidad de cálculo disponible, entre otros. La decisión de analizar un convertidor de tres módulos y siete niveles se justifica en una relación de compromiso entre la complejidad del análisis y el estudio de un convertidor prácticamente realizable. La complejidad de un convertidor de siete niveles es suficiente para justificar un estudio detallado y en profundidad, mientras que las prestaciones a la salida cumplen con los mínimos requerimientos de gran cantidad de aplicaciones de sistemas eléctricos y la industria.

\subsection{Principio de funcionamiento}

El funcionamiento de los convertidores realizados mediante esta topología es esencialmente distinto al de los MVSI más conocidos. Como se esquematiza en la Figura 3.1(a), en los MVSI cada módulo o grupo de llaves y capacitores genera la tensión y corriente de una fase de la carga y por lo tanto pueden ser analizados por separado. En los MCSI simétricos, Figura 3.1(b), cada módulo genera en cada instante corrientes para las tres fases de la carga y por lo tanto no se puede analizar cada fase en forma independiente. Esto implica que la modulación deberá realizarse en forma conjunta y simétrica entre los módulos, y que el ripple de tensión en los inductores divisores dependerá del estado de las llaves de todo el convertidor, la modulación y de las características de la carga. Esto conlleva a un sistema de muy alto orden y completamente acoplado.

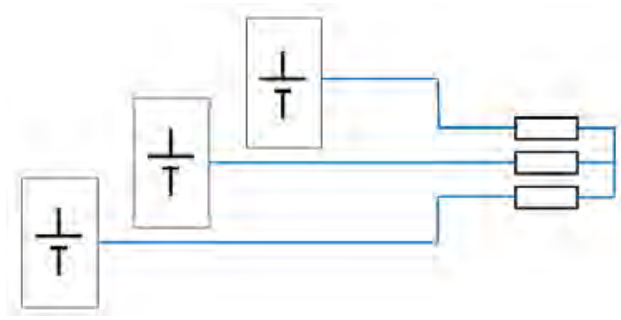

(a) MVSI

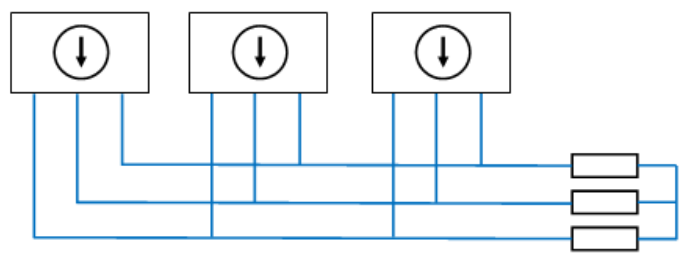

(b) MCSI

Figura 3.1: diferencias en el conexionado del convertidor con la carga.

La principal ventaja de la topología MCSI Simétrico, frente a otros MCSI, es la posibilidad de construcción modular, ya que se puede dividir en partes iguales denominados "módulos" 
cuya combinación constituye el convertidor. En esta topología, cada módulo consiste en dos inductores para dividir la corriente de la fuente principal en partes iguales y un grupo de seis llaves con posibilidad de bloqueo de tensión inversa. Para los niveles de tensión, corriente y frecuencia de trabajo los inductores divisores se dimensionan de manera de tener una corriente casi constante, por lo tanto funcionan como fuentes de corriente. Los inductores divisores conformarán así cada uno de los niveles de corriente en la carga.

En la Figura 3.2 se muestra el diagrama esquemático de un MCSI de tres módulos en el que se utilizan dieciocho llaves con posibilidad de bloqueo bidireccional de tensión que, dependiendo de la potencia y frecuencia requeridas, pueden implementarse mediante transistores MOS, IGBT, ETO, IGCT, entre otras, con el agregado de un diodo ultra-rápido en serie. Cada grupo de seis llaves es alimentado mediante un par de inductores cuya función es la de dividir la corriente de la fuente principal en partes iguales, tres en este caso. De esta forma se tiene la posibilidad de sumar tres fuentes de corriente para generar seis niveles en la carga: $1,2 / 3,1 / 3,-1 / 3,-2 / 3,-1$, con la modulación adecuada puede generarse también corriente nula, resultando un convertidor de siete niveles.

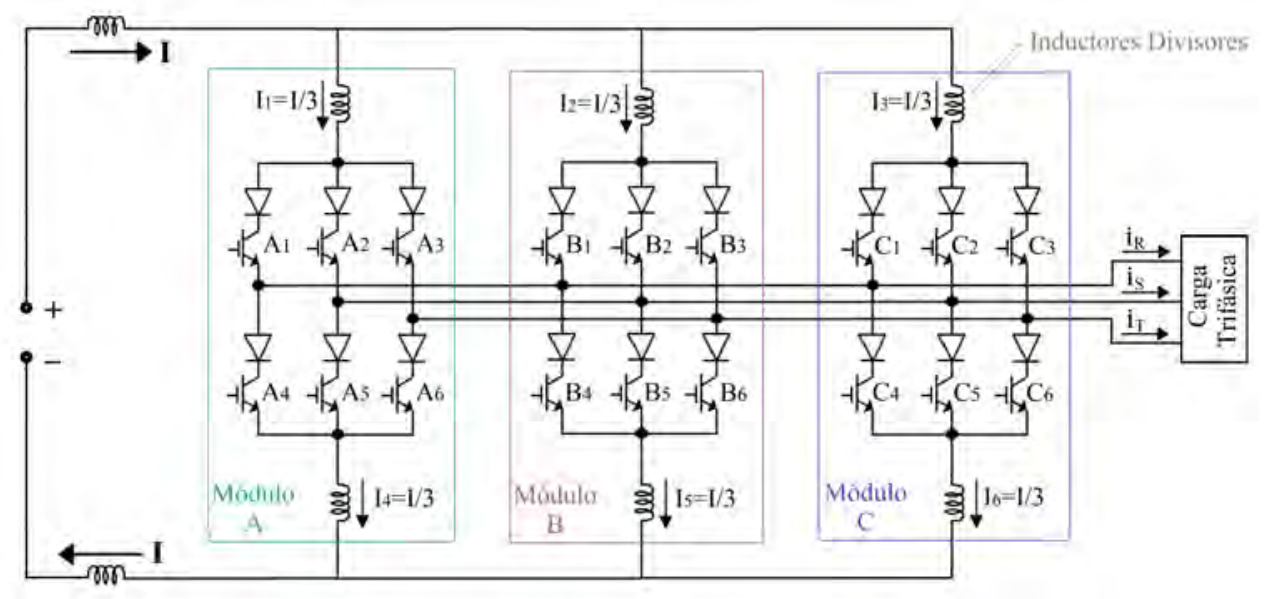

Figura 3.2: esquema circuital del convertidor analizado.

Como en todo convertidor existen ciertas combinaciones de llaves válidas, y otras no, para generar los niveles deseados en la salida.

Las condiciones básicas para generar una combinación válida en un módulo son:

- La corriente de cada inductor del módulo sólo puede ir a una fase de la carga. Lo que implica que en todo instante cada módulo entregará a la carga corriente positiva en una fase, negativa en otra y corriente cero en la restante, Figuras 3.3(b) y 3.3(c).

- Siempre debe estar cerrada una llave del grupo superior, por ejemplo $A_{1}, A_{2}$ o $A_{3}, \mathrm{y}$ una llave del grupo inferior, por ejemplo $A_{4}, A_{5}$ o $A_{6}$. Esto garantiza un camino de circulación a la corriente de los inductores divisores. Si esta condición no se cumple, aparecerá una sobretensión causada por la inductancia a la que se le niegue la circulación de corriente, con efecto destructivo sobre alguna de las llaves asociadas al inductor. 
- Cuando la modulación requiera generar cero corriente en las tres fases de la carga, es posible cerrar el camino de la corriente entre los dos inductores del módulo. A esta acción se la denomina generar un estado cero en la carga, Figura 3.3(d).

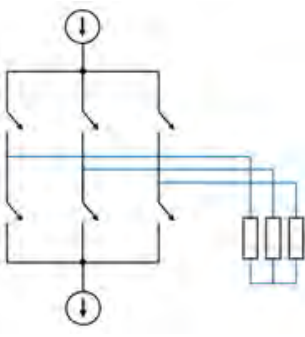

(a)

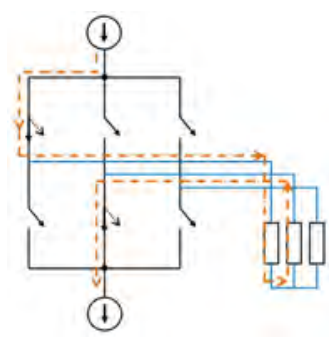

(b)

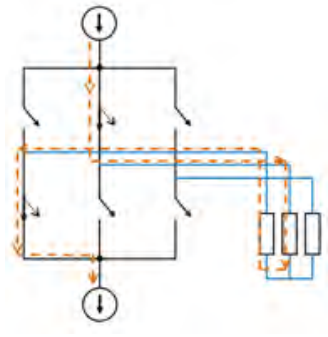

(c)

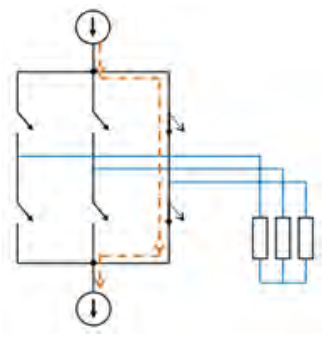

(d)

Figura 3.3: ejemplo de combinaciones válidas de llaves en un módulo. a) esquema básico de un módulo y su conexión con la carga, b) corriente positiva en la fase $\mathrm{R}$ y negativa en $\mathrm{S}, \mathrm{c}$ ) corriente positiva en la fase $\mathrm{S}$ y negativa en $\mathrm{R}$, y d) cero corriente en la carga.

Para generar los siete niveles de corriente en la salida correspondientes al MCSI de tres módulos en estudio existen 729 combinaciones válidas de estados de las llaves, que son aquellas que no violan las leyes de Kirchoff y mantienen el flujo de corriente por cada inductor en todo momento. El número de combinaciones se obtiene de considerar que sólo una llave del conjunto $\left[A_{1}, A_{2}, A_{3}\right]$ puede estar encendida, y para cada una de ellas sólo puede estar encendida una llave del conjunto $\left[A_{4}, A_{5}, A_{6}\right]$, el resultado de 9 combinaciones debe considerarse independiente para cada módulo, ya que al ser totalmente simétricos las combinaciones de llaves son intercambiables, obteniéndose asi $9^{3}$ combinaciones distintas que pueden generar el mismo nivel de corriente en cada fase. A cada combinación válida de llaves se la denomina "estado", a fin de simplificar su referencia en el texto.

Para realizar un primer análisis del funcionamiento del convertidor se selecciona un conjunto de estados que permita generar los niveles de corriente requeridos en la carga para un sistema trifásico balanceado, los que definen una secuencia de encendido de las llaves. En el Cuadro 3.1 se muestra uno de los conjuntos de estados válidos que, además de generar un sistema trifásico de corriente sinusoidal en la carga con los valores del Cuadro 3.2, tiene como características especiales el producir la mínima cantidad de cambios de estado de las llaves por unidad de tiempo y además generar señales de disparo simétricas, minimizando y ecualizando las pérdidas por conmutación en todas las llaves del convertidor (Holmes y Lipo, 2003; Zmood y Holmes, 1998).

$\mathrm{Al}$ recorrer en orden descendente el Cuadro 3.1 se generan seis niveles en cada una de las corrientes de la carga, según el detalle del Cuadro 3.2. Tal como se puede observar en la Figura 3.4, la salida se genera con la frecuencia fundamental deseada, $120^{\circ}$ de desfasaje entre fases y valor pico igual a la corriente principal $I$. Esto permite verificar el funcionamiento básico del convertidor, utilizando la mínima cantidad de conmutaciones en las llaves y analizar en detalle la circulación de corrientes en distintas situaciones.

Las filas 4 y 5 del Cuadro 3.1, que se corresponden con las columnas 4 y 5 del 
Cuadro 3.1: ejemplo de combinaciones válidas de estados de las llaves para un ciclo de corriente de salida.

\begin{tabular}{c|cccccccccccccccccc}
\hline Est. 1 & A1 & A2 & A3 & A4 & A5 & A6 & B1 & B2 & B3 & B4 & B5 & B6 & C1 & C2 & C3 & C4 & C5 & C6 \\
\hline 1 & 1 & 0 & 0 & 0 & 1 & 0 & 0 & 0 & 1 & 0 & 1 & 0 & 0 & 0 & 1 & 0 & 1 & 0 \\
2 & 1 & 0 & 0 & 0 & 1 & 0 & 1 & 0 & 0 & 0 & 1 & 0 & 0 & 0 & 1 & 0 & 1 & 0 \\
3 & 1 & 0 & 0 & 0 & 1 & 0 & 1 & 0 & 0 & 0 & 1 & 0 & 1 & 0 & 0 & 0 & 0 & 1 \\
4 & 1 & 0 & 0 & 0 & 1 & 0 & 1 & 0 & 0 & 0 & 0 & 1 & 1 & 0 & 0 & 0 \\
5 & 0 & 1 & 0 & 0 & 0 & 1 & 1 & 0 & 0 & 0 & 0 & 1 & 1 & 0 & 0 & 0 & 0 \\
6 & 0 & 1 & 0 & 0 & 0 & 1 & 0 & 1 & 0 & 0 & 0 & 1 & 1 & 0 & 0 & 0 & 0 & 1 \\
7 & 0 & 1 & 0 & 0 & 0 & 1 & 0 & 1 & 0 & 0 & 0 & 1 & 0 & 1 & 0 & 1 & 0 & 0 \\
8 & 0 & 1 & 0 & 0 & 0 & 1 & 0 & 1 & 0 & 1 & 0 & 0 & 0 & 1 & 0 & 1 & 0 & 0 \\
9 & 0 & 0 & 1 & 1 & 0 & 0 & 0 & 1 & 0 & 1 & 0 & 0 & 0 & 1 & 0 & 1 & 0 & 0 \\
10 & 0 & 0 & 1 & 1 & 0 & 0 & 0 & 0 & 1 & 1 & 0 & 0 & 0 & 1 & 0 & 1 & 0 & 0 \\
11 & 0 & 0 & 1 & 1 & 0 & 0 & 0 & 0 & 1 & 1 & 0 & 0 & 0 & 0 & 1 & 0 & 1 & 0 \\
12 & 0 & 0 & 1 & 1 & 0 & 0 & 0 & 0 & 1 & 0 & 1 & 0 & 0 & 0 & 1 & 0 & 1 & 0 \\
\hline
\end{tabular}

Cuadro 3.2: corriente de salida para los estados del Cuadro 3.1

\begin{tabular}{c|ccccccccccccc}
\hline Estado & 1 & 2 & 3 & 4 & 5 & 6 & 7 & 8 & 9 & 10 & 11 & 12 \\
\hline$i_{R}$ & $1 / 3$ & $2 / 3$ & 1 & 1 & $2 / 3$ & $1 / 3$ & $-1 / 3$ & $-2 / 3$ & -1 & -1 & $-2 / 3$ & $-1 / 3$ \\
$i_{S}$ & -1 & -1 & $-2 / 3$ & $-1 / 3$ & $1 / 3$ & $2 / 3$ & 1 & 1 & $2 / 3$ & $1 / 3$ & $-1 / 3$ & $-2 / 3$ \\
$i_{T}$ & $2 / 3$ & $1 / 3$ & $-1 / 3$ & $-2 / 3$ & -1 & -1 & $-2 / 3$ & $-1 / 3$ & $1 / 3$ & $2 / 3$ & 1 & 1 \\
\hline
\end{tabular}

Cuadro 3.2, están ejemplificadas en las Figuras 3.5(a) y 3.5(b) y en las Figuras 3.5(c) y 3.5(d) respectivamente, donde se puede observar con mejor detalle el recorrido de la corriente para cada fase y las llaves que intervienen según el estado. En la Figura 3.5(a), las llaves $A 1, B 1$ y $C 1$ están encendidas, cada rama conduce una corriente $1 / 3 I$, por lo que la suma genera una corriente $I$ en la fase $R$. La llave $A 5$ produce una corriente negativa de $1 / 3 I$ en la fase $S$, mientras que las llaves $B 6$ y $C 6$ producen $-2 / 3 I$ en la fase $T$. El análisis de la Figura 3.5(c) es similar, produciendo corrientes positivas de $2 / 3 I$ y $1 / 3 I$ en las fases $R$ y $S$ respectivamente, mientras que por la fase $T$ circula toda la corriente $I$ negativa.

La redundancia de estados para generar los mismos niveles en la carga puede observarse comparando las Figuras 3.5(a) y 3.6, en las cuales se muestra que la misma condición de corriente en la carga puede ser generada con dos combinaciones distintas de llaves.

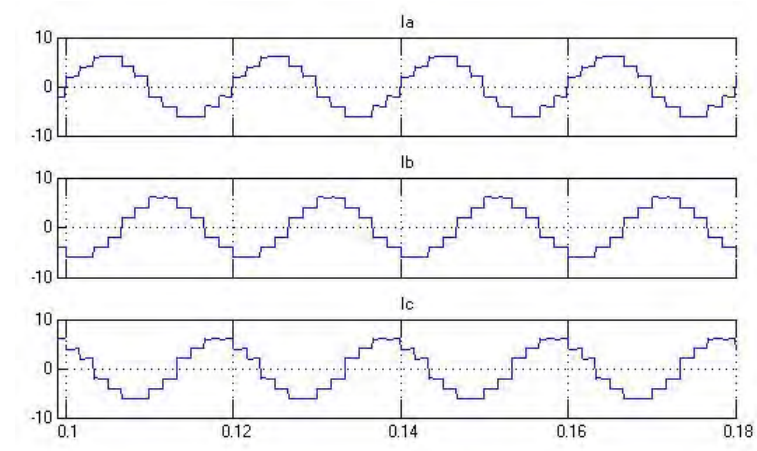

Figura 3.4: corriente de salida multinivel mediante conmutación por tabla. 


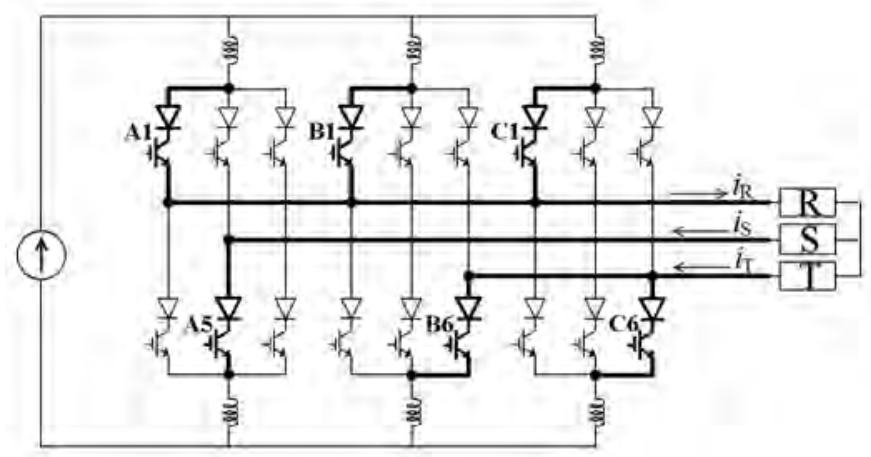

(a) configuración del circuito para el 4to. renglón del Cuadro 3.1 $\left(i_{R}=I, i_{S}=-1 / 3 I, i_{T}=-2 / 3 I\right)$.

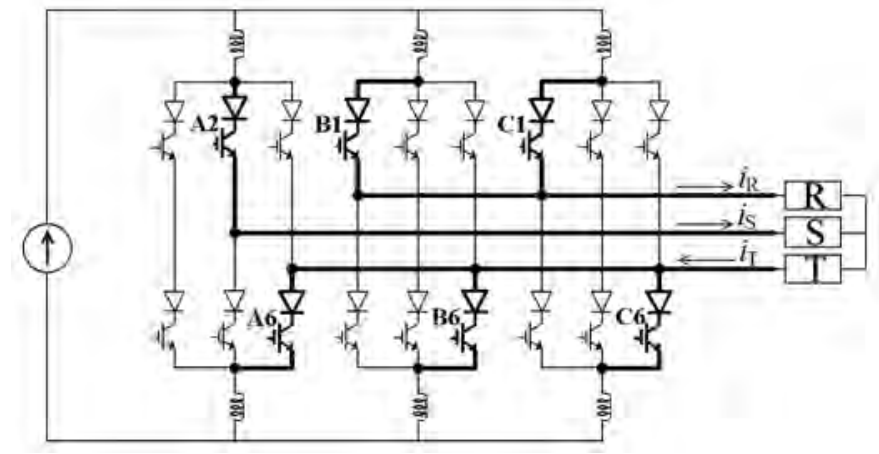

(c) configración del circuito para el 5to. renglón del Cuadro 3.1 $\left(i_{R}=2 / 3 I, i_{S}=1 / 3 I, i_{T}=-I\right)$.

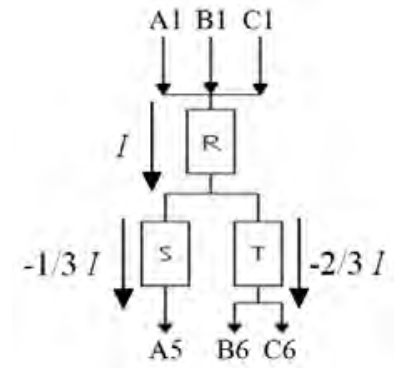

(b)

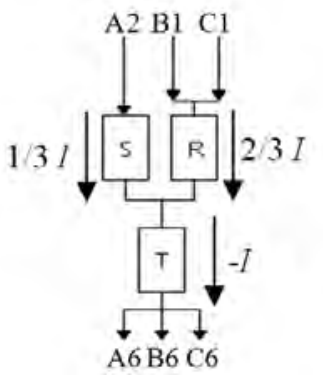

(d)

Figura 3.5: ejemplos de configuraciones circuitales.
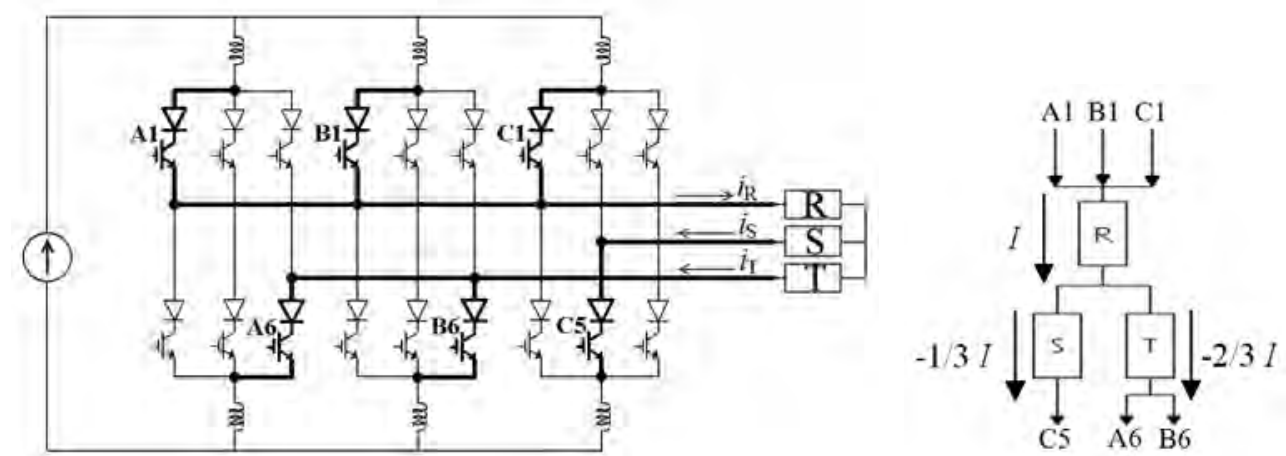

Figura 3.6: configuración equivalente a la Figura 3.5(a).

De la misma forma en que se generó la secuencia del Cuadro 3.1, es posible encontrar una gran cantidad de secuencias de encendido de las llaves, que generen la misma forma de onda de la Figura 3.4. Cada una de estas secuencias tendrá sus características en lo que respecta a frecuencia de conmutación de las llaves, distribución de las corrientes internas, ripple de tensión en los inductores divisores y distribución de pérdidas entre las llaves, entre otros. Pero, para lograr un correcto balance de corrientes en los inductores divisores, se debería analizar 
en cada instante de conmutación el valor de cada corriente interna del convertidor, y el valor de las corrientes y tensiones de salida, para estudiar cuál de las conmutaciones posibles para generar el siguiente valor en la carga producirá el balance deseado. Esto requiere una enorme potencia de cálculo en línea y además medir con bajo error todas las variables del convertidor; ambos requerimientos implican un alto costo y por esta razón se descarta la modulación por tablas. Además, si bien es teóricamente posible obtener una secuencia de conmutación que realice el balance de corrientes, tal como sugiere Xiong et al. (2004a), se ha comprobado en las simulaciones que sólo sirve para condiciones de carga muy específicas y que el balance no soporta perturbaciones, asimetrías en el circuito o dispersión en los tiempos de conmutación.

Además, la modulación mediante tablas no permite realizar las formas de onda con armónicas y/o perturbaciones que debe generar un convertidor real utilizado, por ejemplo, como filtro activo. Es por ello que se realizó un análisis de distintas técnicas de modulación PWM, cuyos resultados se presentan en la sección siguiente.

\subsection{Modulación}

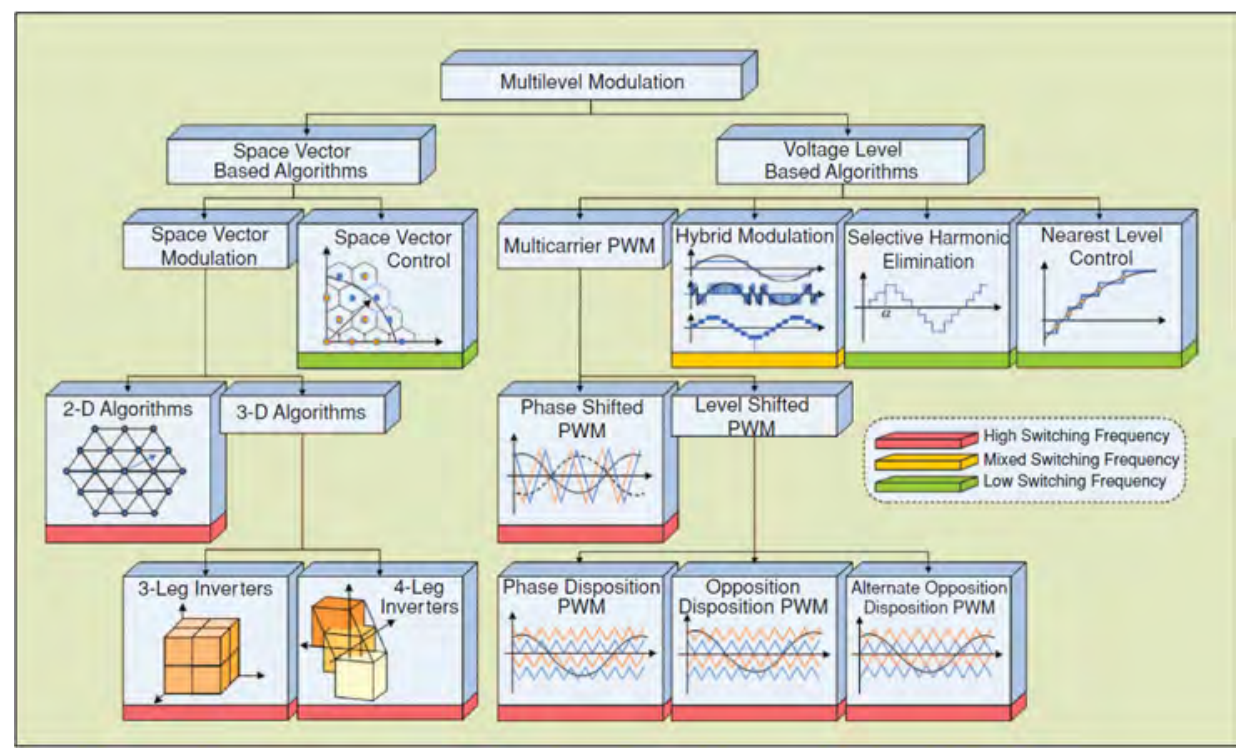

Figura 3.7: modulación de convertidores multinivel (Franquelo et al., 2008).

Existen en la literatura diversas alternativas para la modulación de convertidores multinivel, tal como ejemplifican en su trabajo Franquelo et al. (2008) con el diagrama que se muestra en la Figura 3.7, en el cual se presentan las principales modulaciones para convertidores multinivel, separadas de acuerdo a su principio de funcionamiento. Las dos grandes divisiones en cuanto a algoritmos de modulación están basadas en vector espacial y nivel de la señal de referencia (tensión o corriente). Las modulaciones basadas en vector espacial tienen como principal ventaja el considerar a todo el convertidor en conjunto y por lo tanto permiten optimizar las conmutaciones; pero requieren de una gran potencia de cálculo, mucho mayor aún si de pretende implementar algoritmos de optimización para controlar el 
balance de los elementos de almacenamiento intermedio (capacitores o inductores). Por otro lado, las modulaciones basadas en el nivel de la señal de referencia requieren, en general, menor potencia de cálculo pero tratan a cada parte del convertidor, rama o módulo, por separado y por lo tanto es muy complicado implementar algoritmos que optimicen las conmutaciones de todo el convertidor. Dentro de las modulaciones por nivel de la señal de referencia se encuentran:

- modulación híbrida: se utiliza en convertidores asimétricos, debido a la posibilidad de contar con distintos niveles de comparación.

- eliminación selectiva de armónicos: permite obtener a la salida un espectro bien definido, con la desventaja de requerir una enorme potencia de cálculo, siendo muy difícil su realización en tiempo real.

- control directo de nivel: si bien requiere de mínima potencia de cálculo, es muy complicada la implementación de algoritmos de optimización de conmutaciones y balance de tensiones o corrientes .

- modulaciones por ancho de pulso: están basadas en la tradicional modulación PWM, donde se muestrea, o compara, una señal de referencia con una modulante, o portadora. En el caso de convertidores trifásicos multinivel, se utiliza un sistema trifásico sinusoidal como referencia y una serie de portadoras triangulares desplazadas en tiempo o amplitud.

Dado que en la Sección 3.2 se describieron los problemas y desventajas de utilizar modulación por tablas por la complejidad de su implementación, la necesidad de contar con información del valor de las variables internas del convertidor con alta precisión y la potencia de cómputo requerida, queda como mejor alternativa la utilización de una modulación por ancho de pulso (PWM) y en especial alguna de las que utilizan las técnicas de multiplicidad de portadoras, que se pueden dividir en dos familias: (Holtz, 1992; Holmes y Lipo, 2003)

- con desplazamiento en amplitud: Phase Disposition (PD), Phase Opposition Disposition (POD), Alternative Phase Opposition Disposition (APOD)

- con desplazamiento de fase: Phase Shift Carrier (PSC)

Las modulaciones con desplazamiento de amplitud (PD, POD, APOD) producen un funcionamiento diferenciado de cada parte del convertidor de acuerdo al nivel de la salida y por lo tanto son ideales para los convertidores asimétricos donde se pueden utilizar llaves de distinta tecnología y potencia para generar la corriente de salida, como se presenta en (Dixon y Moran, 2002). Pero, en los convertidores MCSI simétricos esto implicaría que el módulo que responde a la portadora de mayor valor sólo trabajaría con las mayores amplitudes de la corriente de la salida, produciendo distintos niveles de actividad en las llaves del convertidor. Para aprovechar la construcción modular y simétrica de los MCSI bajo estudio es necesario utilizar una modulación que mantenga la simetría entre las distintas partes y 
por ello se propone utilizar la Modulación Sinusoidal de Ancho de Pulso con Portadoras Desfasadas (PSC-SPWM)(Lu et al., 2013). Esta modulación trabaja con portadoras de la misma amplitud, desfasadas simétricamente de acuerdo al número de módulos del convertidor, produciendo niveles simétricos de trabajo en las llaves tanto en corriente, como en el número de conmutaciones por ciclo (equivalente a la frecuencia de conmutación), mejorando el rendimiento global del convertidor. Además, las llaves del convertidor mantienen el mismo nivel de actividad, independientemente del punto de trabajo $\left(m_{a}\right)$ del convertidor.

\subsubsection{Tri-Logic SPWM}

Las modulaciones presentadas en la Figura 3.7 están desarrolladas principalmente para convertidores MVSI, siendo necesaria una adaptación para cumplir con los requerimientos específicos de los MCSI. Para ello se describe en primera instancia la generación de las señales de modulación para un módulo para luego extender el análisis al convertidor completo, siendo los resultados aplicables a convertidores de cualquier número de módulos.

La modulación sinusoidal por ancho de pulso tradicional (SPWM) se basa en la comparación entre una señal de control sinusoidal y una portadora triangular. Las llaves de una rama del convertidor se encienden o apagan dependiendo de si la señal de control sinusoidal asociada a esa rama es mayor o menor que la portadora. Cuando la modulación SPWM se aplica a convertidores de tensión (VSI), las señales de control de cada una de las tres ramas del convertidor, denominadas $P_{R}, P_{S}$ y $P_{T}$ en la Figura 3.8, se generan mediante la comparación de tres señales sinusoidales de control con una portadora triangular. Pero para aplicar la modulación a un MCSI es necesario operar sobre las señales $P_{i}$ ya que además de generar el nivel deseado de corriente en la carga es preciso mantener la circulación de corriente en los inductores divisores en todo momento (Espinoza y Joos, 1993).

Para generar las señales de disparo de las seis llaves de un módulo, se realiza la resta lógica de las señales $P_{i}$, obteniéndose las señales auxiliares $L_{R}, L_{S}$ y $L_{T}$, equivalentes a las tensiones de línea de un VSI. Cada señal $L_{i}$ contiene la información del estado de las llaves de una rama del módulo, pero para su uso directo en el disparo de las llaves primero debe ser decodificada:

- la llave superior de la rama $\left(A_{1}, A_{2}\right.$ o $\left.A_{3}\right)$ se debe encender sólo cuando la referencia correspondiente es mayor que cero, por ejemplo la llave $A_{1}$ se debe encender sólo cuando la referencia $R$ es positiva.

- la llave inferior de la rama $\left(A_{4}, A_{5}\right.$ o $\left.A_{6}\right)$ se debe encender sólo cuando la referencia es menor que cero, por ejemplo la llave $A_{4}$ se debe encender sólo cuando la referencia $R$ es negativa).

La información contenida en las señales $L_{i}$ se puede decodificar mediante las señales $S_{i}$ que corresponden al signo de cada una de las referencias: $S_{R}$ es el signo de la referencia de fase $R, S_{S}$ es el signo de la referencia de fase $S$ y $S_{T}$ es el signo de la referencia de fase $T$. Utilizando las señales de signo se puede derivar la información contenida en las señales $L_{i}$ a 


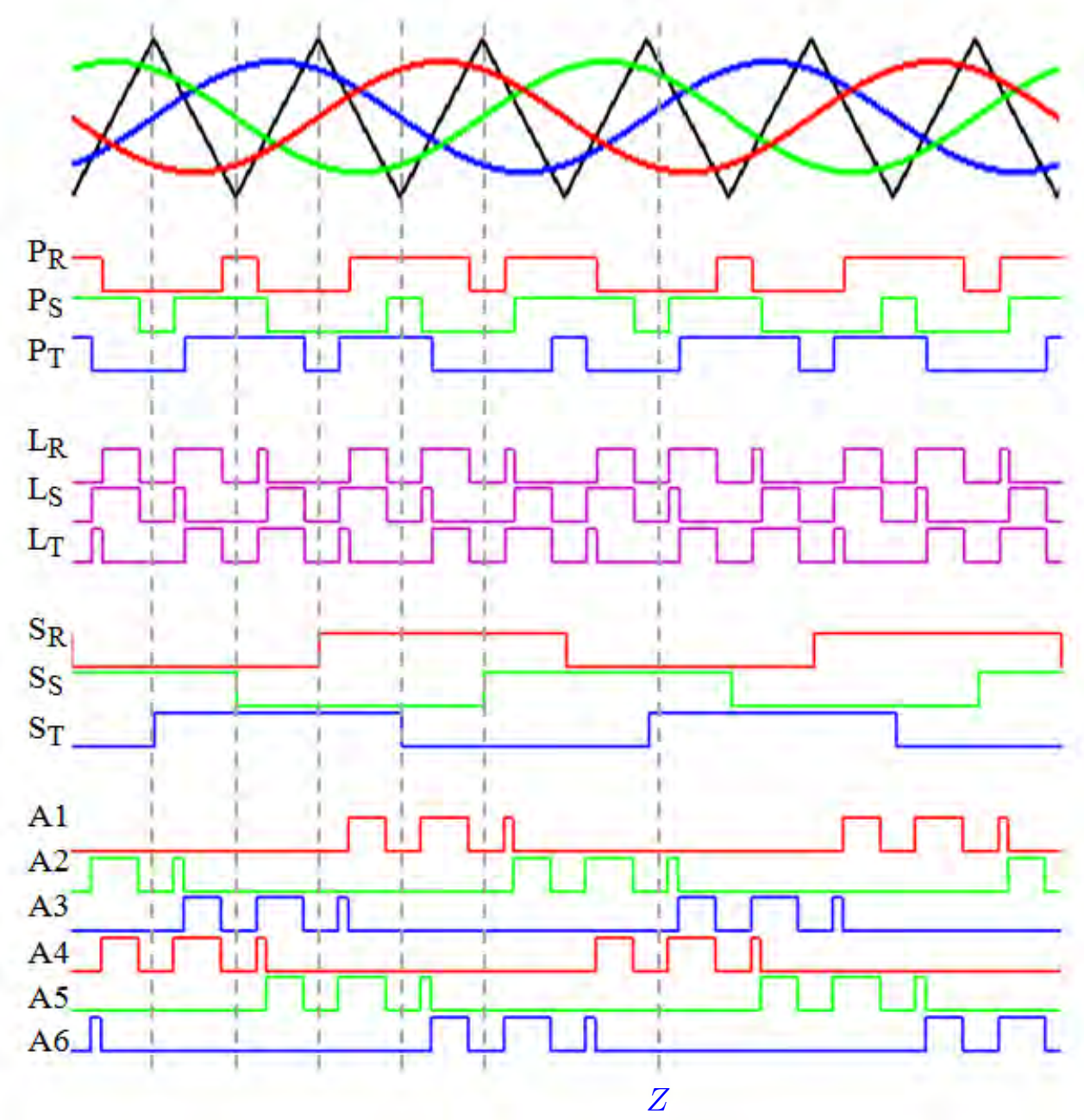

Figura 3.8: señales de la modulación SPWM y Tri-Logic para un módulo.

la llave superior correspondiente cuando la senal de signo es positiva y a la llave inferior en el caso contrario. Las seis señales de control de las llaves de un módulo se obtienen así mediante las expresiones (3.1).

$$
\begin{array}{lll}
A_{1}=\left[P_{R} \oplus P_{S}\right] \odot S_{R} & A_{2}=\left[P_{S} \oplus P_{T}\right] \odot S_{S} & A_{3}=\left[P_{T} \oplus P_{R}\right] \odot S_{T} \\
A_{4}=\left[P_{R} \oplus P_{S}\right] \odot \overline{S_{R}} & A_{5}=\left[P_{S} \oplus P_{T}\right] \odot \overline{S_{S}} & A_{6}=\left[P_{T} \oplus P_{R}\right] \odot \overline{S_{T}}
\end{array}
$$

Esta técnica de modulación se denomina también Tri-Logic SPWM en la literatura (Bai et al., 2008; Wang y Ooi, 1993; Bai y Zhang, 2008b), pero su salida no puede utilizarse directamente en un MCSI ya que, como puede observarse en la Figura 3.8, hay ciertos instantes en los que la modulación apaga las seis llaves del módulo a la vez, no permitiendo la continuidad de corriente en los inductores, algunos ejemplos están marcados con líneas verticales y uno de ellos se ha identificado como tiempo "Z". 


\subsubsection{Estados cero}

En términos de modulación, se denomina comúnmente estado cero a las combinaciones de llaves que generan un valor nulo en la salida, sea cero tensión en un VSI o cero corriente en un CSI. En el caso de un VSI el estado de cero tensión se produce encendiendo las tres llaves superiores, o las tres llaves inferiores, obteniéndose este resultado directamente de la modulación, tal como se puede observar en el tiempo "Z" de la Figura 3.8 donde un valor cero en las tres señales $P_{i}$ implica que las tres llaves inferiores del convertidor estarán encendidas. Para un CSI, los estados cero son aquellos que entregan un valor nulo de corriente en la carga, pero no es posible apagar la totalidad de las llaves del módulo, como se obtiene de aplicar directamente la modulación Tri-Logic, ya que esa situación implica que se estaría interrumpiendo el camino de circulación de la corriente de los inductores divisores y ello llevaría a una situación destructiva. Por ello es preciso detectar los estados cero generados por la modulación y reemplazarlos por estados cero válidos para el MCSI.

Cada módulo puede generar estados cero de corriente en la carga de tres formas distintas, como se muestra en la Figura 3.9. La forma que requiere la menor potencia de cálculo es aquella en la que se encienden las seis las llaves del módulo a la vez, 3.9(a), permitiendo la libre circulación de la corriente de los inductores divisores; pero esta solución implica mayores pérdidas de conmutación. También es posible encender cuatro llaves del módulo, permitiendo un doble camino para la corriente de las inductancias divisoras, 3.9(b), pero la solución más efectiva es encender las dos llaves de una rama, lo que implica los tres estados cero equivalentes por módulo de la Figura 3.9(c).

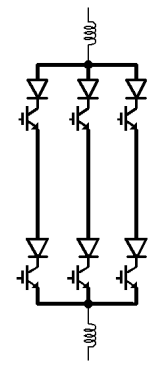

(a)

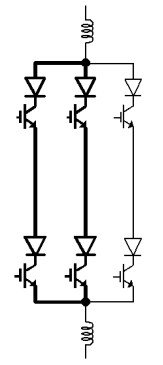

(b)

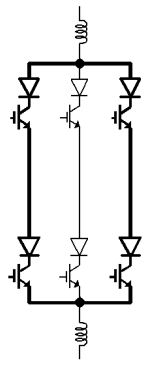

b)

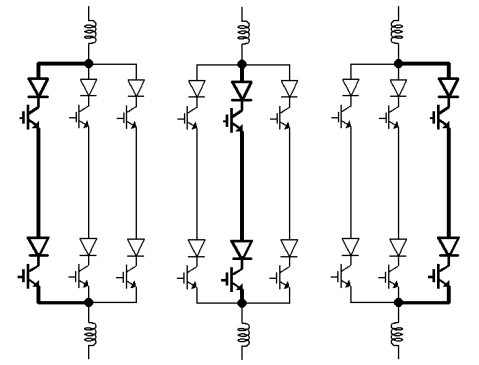

(c)

Figura 3.9: combinaciones posibles de estados cero.

El encender sólo dos llaves para generar un estado cero permite una redundancia en las combinaciones de llaves que pueden producir un estado cero de corriente en la carga y por lo tanto puede ser aprovechada para reducir el número de conmutaciones por ciclo de las llaves y así minimizar las pérdidas del convertidor (Wang y Ooi, 1993; Zmood y Holmes, 1998).

\subsubsection{Minimización de la frecuencia de conmutación}

Haciendo uso de la redundancia de estados cero que posee la topología MCSI Simétrico, se analiza el resultado de la modulación a fin de detectar cada estado cero inválido generado por 
la PSC-SPWM y reemplazarlo por un estado cero válido propio del convertidor, y cumplir así con las condiciones de continuidad de corriente. Para ello se analizan en detalle las señales de conmutación $\left(A_{1} \ldots A_{6}\right)$ generadas por la modulación y se identifican seis estados activos, que se muestran en el Cuadro 3.3, los que generan todas las combinaciones de la corriente $I / 3$ que puede entregar el módulo a la carga.

Cuadro 3.3: estados válidos de las llaves generados por la modulación PSC-SPWM.

\begin{tabular}{c|cccccc|ccc}
\hline Estado & $A_{1}$ & $A_{2}$ & $A_{3}$ & $A_{4}$ & $A_{5}$ & $A_{6}$ & $i_{R}$ & $i_{S}$ & $i_{T}$ \\
\hline 1 & & 1 & & & & 1 & 0 & $\mathrm{I} / 3$ & $-\mathrm{I} / 3$ \\
2 & 1 & & & & 1 & & $\mathrm{I} / 3$ & $-\mathrm{I} / 3$ & 0 \\
3 & 1 & & & & & 1 & $\mathrm{I} / 3$ & 0 & $-\mathrm{I} / 3$ \\
4 & & & 1 & 1 & & & $-\mathrm{I} / 3$ & 0 & $\mathrm{I} / 3$ \\
5 & & 1 & & 1 & & & $-\mathrm{I} / 3$ & $\mathrm{I} / 3$ & 0 \\
6 & & & 1 & & 1 & & 0 & $-\mathrm{I} / 3$ & $\mathrm{I} / 3$ \\
\hline
\end{tabular}

Además se pueden identificar seis secuencias en las cuales se presentan los estados activos:

$$
\begin{aligned}
& \text { Secuencia } A: 6 \rightarrow 2 \rightarrow 0 \rightarrow 2 \rightarrow 6 \rightarrow 0 \rightarrow 6 \rightarrow 2 \rightarrow \ldots \\
& \text { SecuenciaB }: 2 \rightarrow 3 \rightarrow 0 \rightarrow 3 \rightarrow 2 \rightarrow 0 \rightarrow 2 \rightarrow 3 \rightarrow \ldots \\
& \text { SecuenciaC }: 3 \rightarrow 1 \rightarrow 0 \rightarrow 1 \rightarrow 3 \rightarrow 0 \rightarrow 3 \rightarrow 1 \rightarrow \ldots \\
& \text { SecuenciaD }: 1 \rightarrow 5 \rightarrow 0 \rightarrow 5 \rightarrow 1 \rightarrow 0 \rightarrow 1 \rightarrow 5 \rightarrow \ldots \\
& \text { SecuenciaE }: 5 \rightarrow 4 \rightarrow 0 \rightarrow 4 \rightarrow 5 \rightarrow 0 \rightarrow 5 \rightarrow 4 \rightarrow \ldots \\
& \text { SecuenciaF }: 4 \rightarrow 6 \rightarrow 0 \rightarrow 6 \rightarrow 4 \rightarrow 0 \rightarrow 4 \rightarrow 6 \rightarrow \ldots
\end{aligned}
$$

Estas seis secuencias se repiten cíclicamente como se muestra en la Figura 3.10. La modulación genera una de esas secuencias de estados que se mantiene durante un cierto tiempo, dependiendo de la forma de las señales de referencia (sinusoidales) y su relación con la portadora triangular. A medida que avanza el tiempo y las referencias cambian, siguiendo una forma sinusoidal u otra, las comparaciones entre referencia y portadora se modifican y se comienza a generar otra secuencia de estados, lo cual genera un nuevo conjunto de valores de corriente a la salida. Dado que la forma de onda de la corriente de salida será fundamentalmente sinusoidal, con el futuro agregado de armónicos y distorsiones, es posible identificar el orden en el que se repetirán las secuencias, resultando en el diagrama secuencial (también conocido como máquina secuencial) de la Figura 3.10.

Esta característica de la modulación PSC-SPWM permite construir una máquina secuencial lógica que detecte la secuencia de estados que está generando el módulo bajo análisis, para asi buscar e identificar los estados cero y reemplazarlos por estados válidos del convertidor utilizando una configuración de salidas tipo Mealy (Wakerly, 2006). Cuando la máquina secuencial detecta la secuencia en la que se está trabajando, por ejemplo la secuencia B, los estados válidos en su entrada serán el 2 y el 3 (Cuadro 3.3). La máquina secuencial permanecerá en esa secuencia hasta que se detecte la siguiente, mediante la llegada de un estado 1 a la entrada. Cada vez que se detecte a la entrada un estado cero será posible 


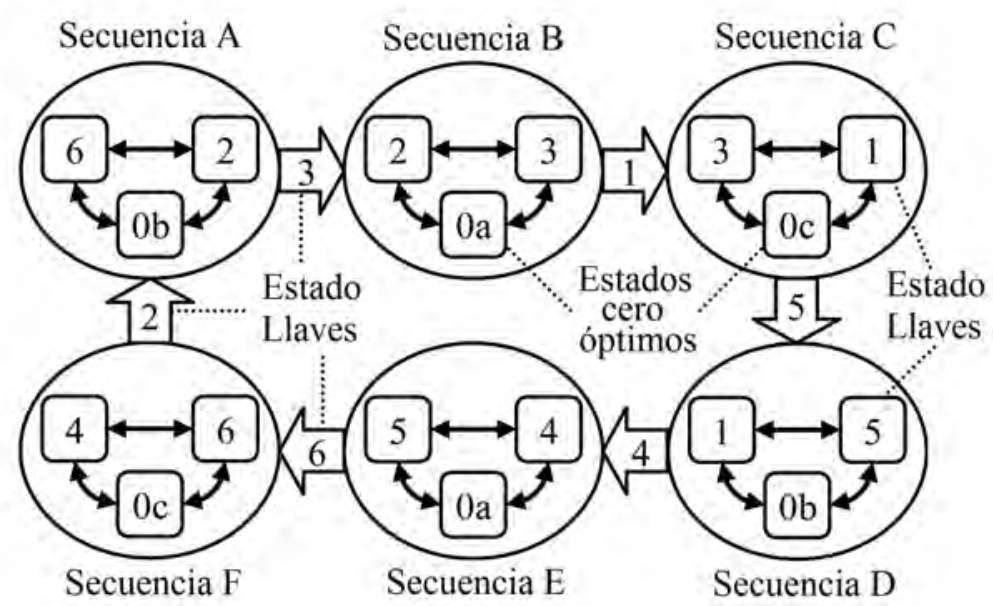

Figura 3.10: máquina secuencial para la selección del estado cero óptimo.

reemplazarlo por aquel estado válido del convertidor que mejor se adecue a los requerimientos de la modulación. En particular, los estados denominados $0_{a}, 0_{b}$ y $0_{c}$, que se muestran en el Cuadro 3.4, cumplen con la condición de generar la mínima cantidad de conmutaciones de las llaves por ciclo, permitiendo maximizar el rendimiento del convertidor y reducir las emisiones electromagnéticas (EMI).

Cuadro 3.4: secuencias de conmutación y estados zero óptimos.

\begin{tabular}{|c|c|c|c|c|c|c|c|}
\hline Secuencia & Estado & A1 & A2 & A3 & A4 & A5 & A6 \\
\hline & 2 & 1 & & & 0 & 1 & 0 \\
\hline \multirow[t]{3}{*}{$\mathbf{A}$} & 6 & & & 1 & 0 & 1 & 0 \\
\hline & $0 \mathrm{~b}$ & & 1 & & 0 & 1 & 0 \\
\hline & 2 & 1 & 0 & 0 & & 1 & \\
\hline \multirow[t]{3}{*}{ B } & 3 & 1 & 0 & 0 & & & 1 \\
\hline & $0 \mathrm{a}$ & 1 & 0 & 0 & 1 & & \\
\hline & 3 & 1 & & & 0 & 0 & 1 \\
\hline \multirow[t]{3}{*}{ C } & 1 & & 1 & & 0 & 0 & 1 \\
\hline & $0 \mathrm{c}$ & & & 1 & 0 & 0 & 1 \\
\hline & 1 & 0 & 1 & 0 & & & 1 \\
\hline \multirow[t]{3}{*}{ D } & 5 & 0 & 1 & 0 & 1 & & \\
\hline & $0 \mathrm{~b}$ & 0 & 1 & 0 & & 1 & \\
\hline & 5 & & 1 & & 1 & 0 & 0 \\
\hline \multirow[t]{3}{*}{$\mathbf{E}$} & 4 & & & 1 & 1 & 0 & 0 \\
\hline & $0 \mathrm{a}$ & 1 & & & 1 & 0 & 0 \\
\hline & 4 & 0 & 0 & 1 & 1 & & \\
\hline \multirow[t]{2}{*}{$\mathbf{F}$} & 6 & 0 & 0 & 1 & & 1 & \\
\hline & $0 \mathrm{c}$ & 0 & 0 & 1 & & & 1 \\
\hline
\end{tabular}

Como puede observarse en las celdas sombreadas del Cuadro 3.4, la elección de los estados cero mencionados permite evitar que algunas llaves conmuten mientras la modulación se encuentra generando una secuencia dada, por ejemplo para la secuencia B, mientras las llaves 
$A_{4}, A_{5}$ y $A_{6}$ conmutan, la llave $A_{1}$ permanece encendida todo el tiempo permitiendo que las llaves $A_{2}$ y $A_{3}$ estén apagadas durante todo el período. De esta manera, a la salida de la máquina secuencial se tendrá:

- el mismo estado generado por la modulación, cuando a la entrada se detecte uno de los estados activos del Cuadro 3.3

- un estado cero válido de acuerdo a la secuencia activa, cuando a la entrada se detecte un estado cero.

La relación entre los estados de entrada y de salida de la máquina secuencial se resumen en la Figura 3.5 para cada una de las secuencias posibles.

$$
\begin{array}{ccc}
2 \longrightarrow 2 & 2 \longrightarrow 2 & 3 \longrightarrow 3 \\
6 \longrightarrow 6 & 3 \longrightarrow 3 & 1 \longrightarrow 1 \\
0 \longrightarrow 0 b & 0 \longrightarrow 0 a & 0 \longrightarrow 0 c
\end{array}
$$

secuencia A secuencia B secuencia C

$\begin{array}{ccc}1 \longrightarrow 1 & 5 \longrightarrow 5 & 4 \longrightarrow 4 \\ 5 \longrightarrow 5 & 4 \longrightarrow 4 & 6 \longrightarrow 6 \\ 0 \longrightarrow 0 b & 0 \longrightarrow 0 a & 0 \longrightarrow 0 c\end{array}$

secuencia D secuencia E secuencia F

Cuadro 3.5: relación entre entrada y salida de la máquina secuencial, para cada secuencia de estados.

Tanto la lógica de modulación, como la máquina secuencial pueden ser implementados en una FPGA, permitiendo su procesamiento en paralelo y en forma segura, evitando los errores por interferencias en la programación que pueden darse en los Microcontroladores y DSP cuando están sometidos a altos niveles de interferencia electromagnética (EMI) como los generados por un MCSI.

La Figura 3.11 permite comparar las señales de disparo de la llave $A_{1}$, siendo muy notoria la reducción de conmutaciones por ciclo lograda mediante la elección del estado cero óptimo realizada por la máquina secuencial.

\subsubsection{Modulación PSC-SPWM para un MCSI de tres módulos}

Para lograr una corriente multinivel a la salida del convertidor es necesario conectar varios módulos y cada uno de ellos dispondrá de su propia lógica SPWM y su máquina secuencial, favoreciendo el procesamiento en paralelo de las señales de control. La modulación PSCSPWM implica que todas las portadoras tienen que estar desfasadas simétricamente dentro de $\operatorname{los} 360^{\circ}$ o $2 \pi$ radianes de un ciclo, por lo que el ángulo de fase $\varphi_{k}$ de cada módulo resulta,

$$
\varphi_{k}=2 \pi k / m \quad k=1,2, \ldots, m
$$




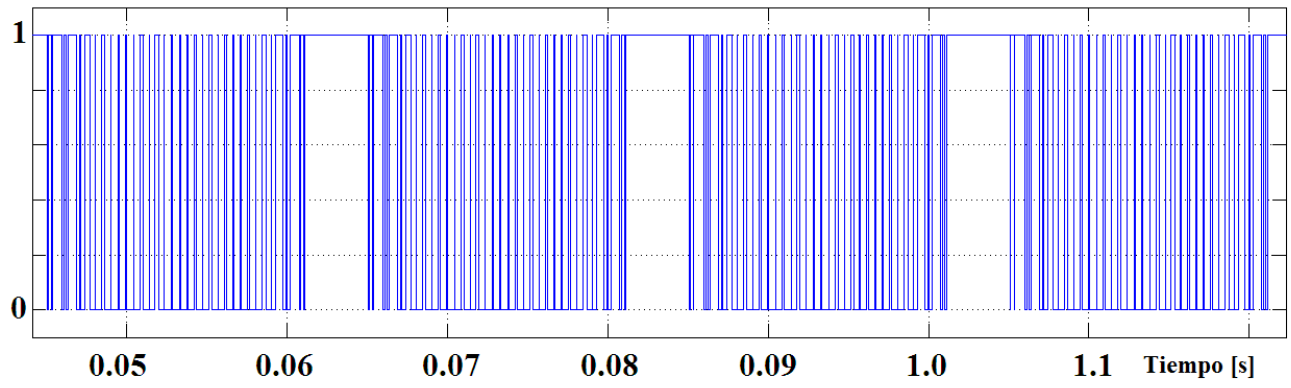

(a)

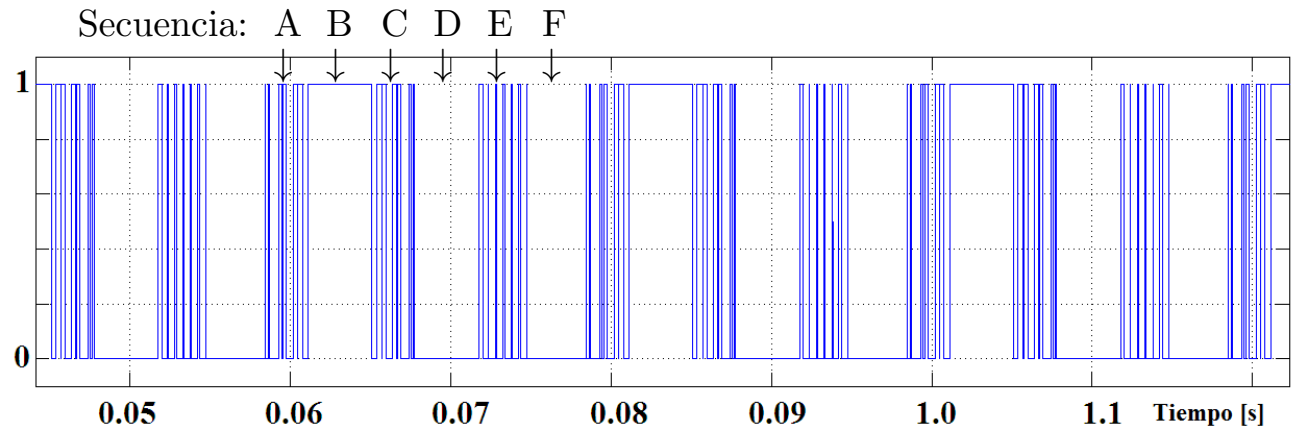

(b)

Figura 3.11: efecto de la máquina de estado en las señales de disparo (llave $A_{1}$ ), a) modulación SPWM original, b) con la máquina secuencial.

donde $m$ es el número de módulos. Para un convertidor de tres módulos las portadoras deben tener $120^{\circ}$ de fase entre ellas, como se muestra en la Figura 3.12. En forma similar a lo ocurrido para los MVSI, la frecuencia de conmutación efectiva en la carga será $m F$, donde $F$ es la frecuencia de portadora de cada módulo, corriendo el espectro de conmutación hacia altas frecuencias. Esta característica permite una rápida respuesta dinámica y un menor requerimiento de los filtros de salida, siendo una de las principales ventajas de las topologías multinivel.

En la Figura 3.13 se puede observar un diagrama esquemático del conexionado de la lógica de modulación y máquina secuencial para un MCSI Simétrico de tres módulos y siete niveles.

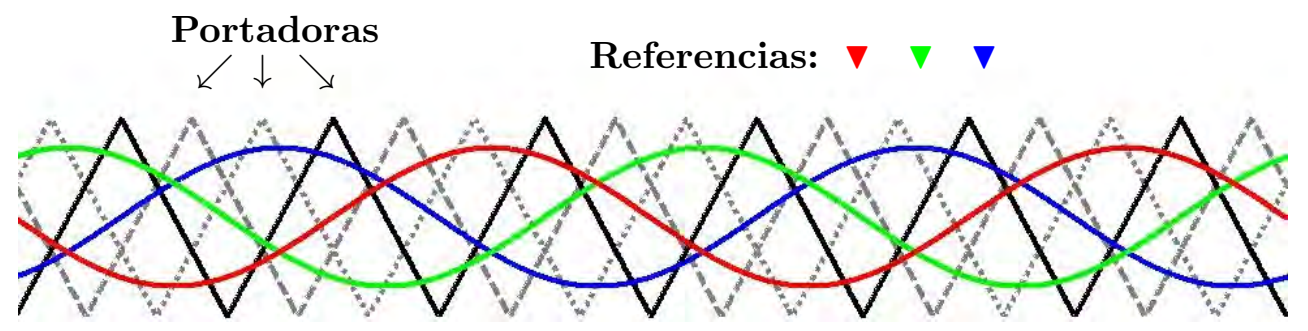

Figura 3.12: modulación PSC-SPWM para un MCSI de tres módulos. 


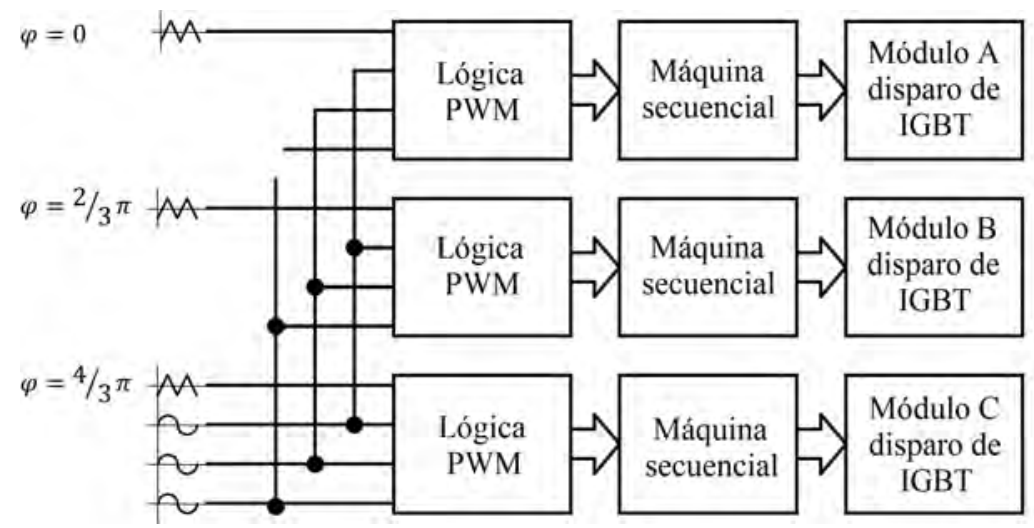

Figura 3.13: diagrama esquemático de la modulación aplicada al convertidor completo.

\subsubsection{Variación del índice de modulación}

En la mayoría de las topologías, tanto MVSI como MCSI, algunas llaves funcionan sólo cuando se deben generar los niveles más altos a la salida y aquellas que están más cerca del punto medio de tensión o de cero corriente funcionan continuamente y a alta frecuencia (Dixon y Moran, 2002), lo que produce algunos problemas que complican la realización de convertidores de alta potencia en la práctica, como por ejemplo: un desbalance en las pérdidas genera diferencias de temperatura entre las llaves y por lo tanto se alteran los tiempos de conmutación causando distorsiones en la salida y mayores corrientes de corto-circuito.

La combinación de la topología MSCI Simétrico con la modulación PSC-SPWM permite distribuir el régimen de trabajo en forma uniforme entre todas las llaves, independientemente del punto de trabajo del convertidor. Cuando se está trabajando con un bajo valor de $\mathrm{m}_{a}$ todas las llaves realizan la misma cantidad de conmutaciones por unidad de tiempo y encienden con el mismo ciclo de trabajo promedio, ya que el menor valor de corriente de salida se consigue generando los estados cero durante una mayor parte del período.

Como se muestra en la Figura 3.14(a), es posible reducir la corriente de salida, mediante el índice de modulación $\left(m_{a}\right)$, pero sin perder la cantidad de pulsos que conforman cada fase. Para lograr una disminución en la amplitud de la corriente de salida se debe aumentar el peso relativo de los estados cero, haciendo que la corriente generada por cada módulo se intercale en el tiempo en vez de sumarse. De esta manera, para un nivel de salida bajo, todos los módulos seguirán aportando corriente en la misma proporción, pero cada uno lo hará mientras los otros están entregando corriente cero. Se construye asi la forma de onda de la derecha de la Figura 3.14(a) donde se observa una gran cantidad de pulsos, pero todos de $1 / 3$ de la corriente principal. La frecuencia equivalente de conmutación en la carga se mantiene y por lo tanto la precisión con la que se genera la forma de onda requerida, por lo que se disminuye notoriamente la distorsión armónica en la salida, manteniendo a todas las llaves del convertidor conmutando con el mismo régimen de trabajo.

En la Figura 3.14(b) se muestran en detalle las señales de disparo de la primer llave de cada módulo $\left(A_{1}, B_{1}\right.$ y $\left.C_{1}\right)$ en dos condiciones de trabajo distintas: a la izquierda para 


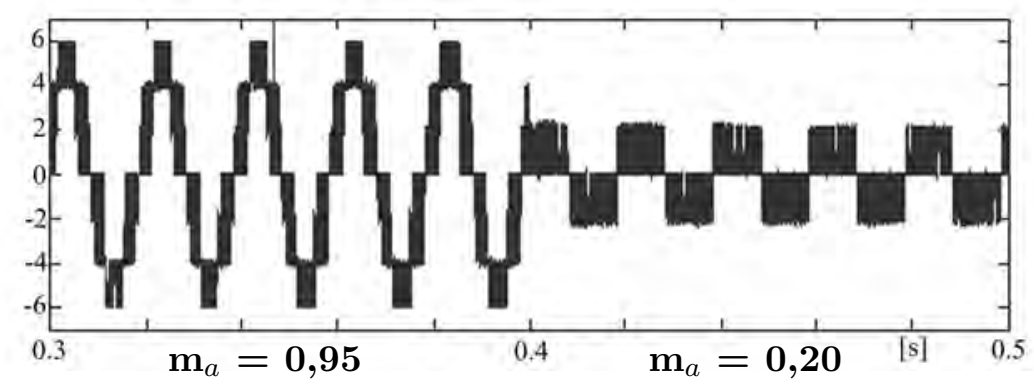

(a) Corriente de salida

Llave 1 - Módulo A

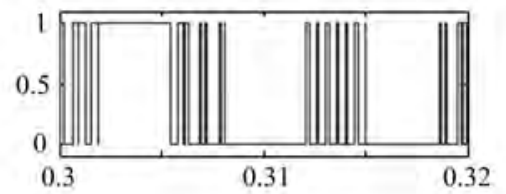

Llave 1 - Módulo B

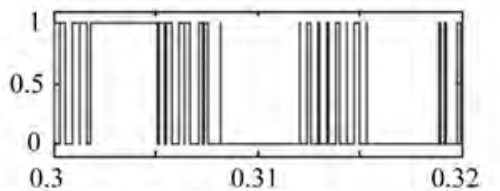

Llave 1 - Módulo C

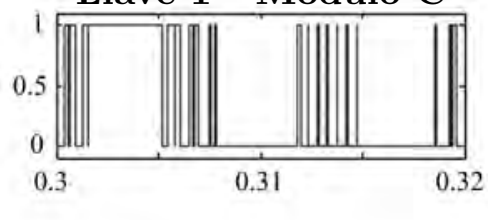

$\mathbf{m}_{a}=0,95$
Llave 1 - Módulo A

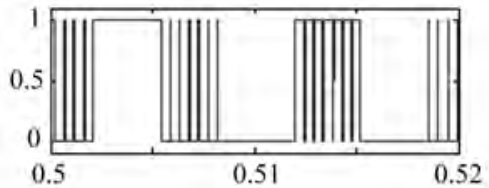

Llave 1 - Módulo B

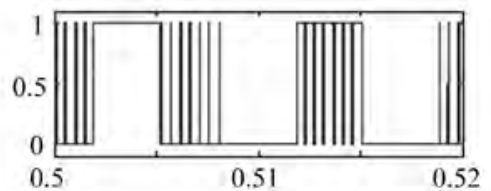

Llave 1 - Módulo C

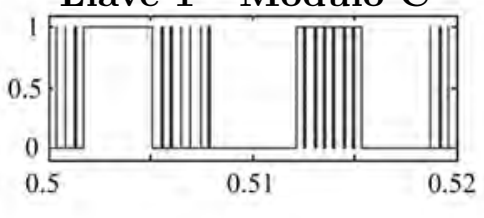

$\mathbf{m}_{a}=\mathbf{0 , 2 0}$

(b) Señales de disparo de la llave 1 de cada módulo

Figura 3.14: comportamiento del MCSI para $\mathrm{m}_{a}=0,95 \mathrm{y}_{a}=0,2$.

un nivel alto de salida $\left(m_{a}=0,95\right)$ y a la derecha para un nivel casi mínimo en la salida $\left(m_{a}=0,2\right)$. Se pueden obtener dos conclusiones principales:

- la cantidad de conmutaciones por ciclo de una llave es independiente del valor de la corriente de salida generada por el convertidor

- las llaves de todos los módulos tienen el mismo nivel de actividad por lo que las pérdidas, tanto por conmutación como por conducción, se distribuyen uniformemente. Esto permite minimizar las pérdidas del convertidor y aumentar la confiabilidad o MTBF. 


\subsection{Eficiencia y Conmutación suave}

Diversos factores afectan la especificación de un convertidor, tales como los niveles de potencia y corriente, frecuencia de conmutación de las llaves, tecnología de las llaves, velocidad de cálculo disponible, prestaciones a la salida, tamaño, capacidad de disipación de calor en el ambiente, seguridad y facilidad de mantenimiento, entre muchos otros. Todos estos factores deben ser tenidos en cuenta para sopesar la relación entre la aplicación, la tecnología a utilizar y el costo de diseño e implementación y obtener así la mejor solución costo-beneficio. Entre ellos, la eficiencia de un convertidor es un factor muy importante a tener en cuenta a la hora de su diseño y/o especificación (Suh et al., 2007; Jalakas et al., 2008). La eficiencia dependerá básicamente de dos grandes factores: a) pérdidas en los componentes pasivos y conductores, b) pérdidas en las llaves.

El primer caso implica un lógico compromiso con el costo, peso y volumen admisibles para el convertidor ya que para reducir las pérdidas en los conductores se debe minimizar la resistencia de los cables y utilizar, para los inductores, núcleos de aleaciones de alto rendimiento con un gran entrehierro. En la topología seleccionada circula sólo una fracción de la corriente total por los inductores y llaves de cada módulo, por lo que las pérdidas por efecto resistivo en los cables, que dependen del cuadrado de la corriente, se ven notablemente reducidas. Además, la frecuencia de conmutación de cada módulo es también una fracción de la frecuencia efectiva total vista por la carga y los filtros, esto implica que las pérdidas en los núcleos de los inductores divisores, tanto por histéresis como por corrientes parásitas (Foucault), se reducen notablemente en comparación con otras topologías. En aplicaciones especiales se puede optar incluso por la tecnología de superconductores (Xiong et al., 2004b).

El segundo caso, las pérdidas en las llaves, es un factor que depende de gran número de variables, entre las que se encuentran las tecnologías de llaves disponibles, nivel de EMI aceptado, e incluso las prestaciones dinámicas en la carga, que generan una relación de compromiso directa con la frecuencia de conmutación. El problema de las pérdidas en las llaves es muy importante, ya que no sólo afecta al rendimiento energético en forma directa, sino que influye en muchos otros aspectos, como ser: volumen del convertidor, tamaño de las llaves, tecnología de enfriamiento y tipo de acople mecánico al disipador. Por ejemplo, una gran disipación de potencia implicará que el convertidor ocupará un gran volumen para poder conducir el calor generado y disiparlo en la atmósfera, ello implicará largos conductores, con un aumento en las componentes parásitas, principalmente inductancia y capacidad, que afectarán en mayor o menor medida, dependiendo de la topología adoptada, el funcionamiento ideal del convertidor. Las pérdidas en las llaves pueden dividirse en:

- pérdidas estáticas: dependen de las características de las llaves en los estados de conducción y corte. Para una potencia dada, son básicamente función del costo y tamaño aceptables del convertidor,

- pérdidas dinámicas: están asociadas directamente con la conmutación de las llaves y dependen principalmente de la tecnología utilizada, su circuito de control y, en algunos casos, del circuito o topología del convertidor y por lo tanto el análisis de las formas 
para su reducción o minimización es de especial interés en este trabajo.

Para la mayoría de las tecnologías de llaves disponibles en la actualidad para convertidores de alta potencia (IGBT, IGCT, ETO, etc.) los tiempos de encendido son inferiores a los tiempos de apagado, debido principalmente a la necesidad de vaciar de portadores la zona $n^{-}$ de doble inyección durante el apagado (Mohan et al., 2005; Li et al., 2009). Esta característica ha sido siempre un inconveniente en el diseño y operación de los MVSI, ya que obliga a mantener un tiempo muerto en la conmutación a fin de evitar que las dos llaves de una rama conduzcan a la vez y ello provoque un cortocircuito que en el mejor de los casos sólo afecta el rendimiento y las prestaciones, pero que en la mayoría puede llegar a ser destructivo. Por otro lado, la necesidad de garantizar un tiempo muerto entre conmutaciones puede producir a la salida de los MVSI efectos no deseados y/o complicar enormemente el control, encontrándose en la literatura numerosas técnicas para minimizar sus efectos negativos (Park et al., 2008; Blahnik et al., 2012).

Para realizar un análisis de la conmutación entre dos llaves cualquiera de los MCSI bajo estudio, es preciso reconocer primero que la conmutación de corriente no se realiza entre las dos llaves de una rama, en la forma tradicional de un MVSI, sino que la corriente se conmuta entre dos de las tres llaves asociadas a cada uno de los inductores divisores, como se muestra en la Figura 3.15. Debe considerarse además que dada la total simetría de construcción y funcionamiento entre módulos, y llaves, de esta topología, se simplifica el análisis de la conmutación ya que todas las llaves operan de la misma forma y con los mismos niveles de corriente y tensión. En este caso no sólo es deseable, sino indispensable, garantizar un solapamiento en los tiempos de conducción de dos llaves que conmuten en secuencia, a fin de asegurar en todo momento la circulación de la corriente de los inductores divisores. El solapamiento resultante produce una conmutación suave de la corriente de dos llaves, como se ejemplifica en la Figura 3.15, donde se puede observar que:

- Antes de iniciarse la conmutación el módulo se encuentra en un estado estable, habiendo pasado un tiempo considerable desde la última conmutación. En el estado de ejemplo, el transistor $T_{1}$ está conduciendo y el transistor $T_{2}$ está apagado. En este caso particular la corriente de los inductores divisores circula sólo a través de las llaves, sin llegar a la carga, pero el análisis siguiente es independiente del estado en el que se encuentre el convertidor.

- La orden de conmutación se envía al mismo tiempo a los dos circuitos de disparo: apagar el transistor $T_{1}$ y encender el transistor $T_{2}$. Pero $T_{1}$ requiere de un tiempo mayor para apagarse del que $T_{2}$ necesita para encenderse, produciéndose una distribución momentánea de la corriente del inductor entre ellos. La distribución de la corriente durante el corto tiempo que dura la conmutación está determinada principalmente por las inductancias parásitas en los caminos de conducción de cada llave.

- Cuando el transistor $T_{1}$ comienza a apagarse, el transistor $T_{2}$ ya está completamente encendido, por lo que la corriente del inductor divisor puede circular libremente a través de $T_{2}$, produciendo un apagado sin sobretensiones. 

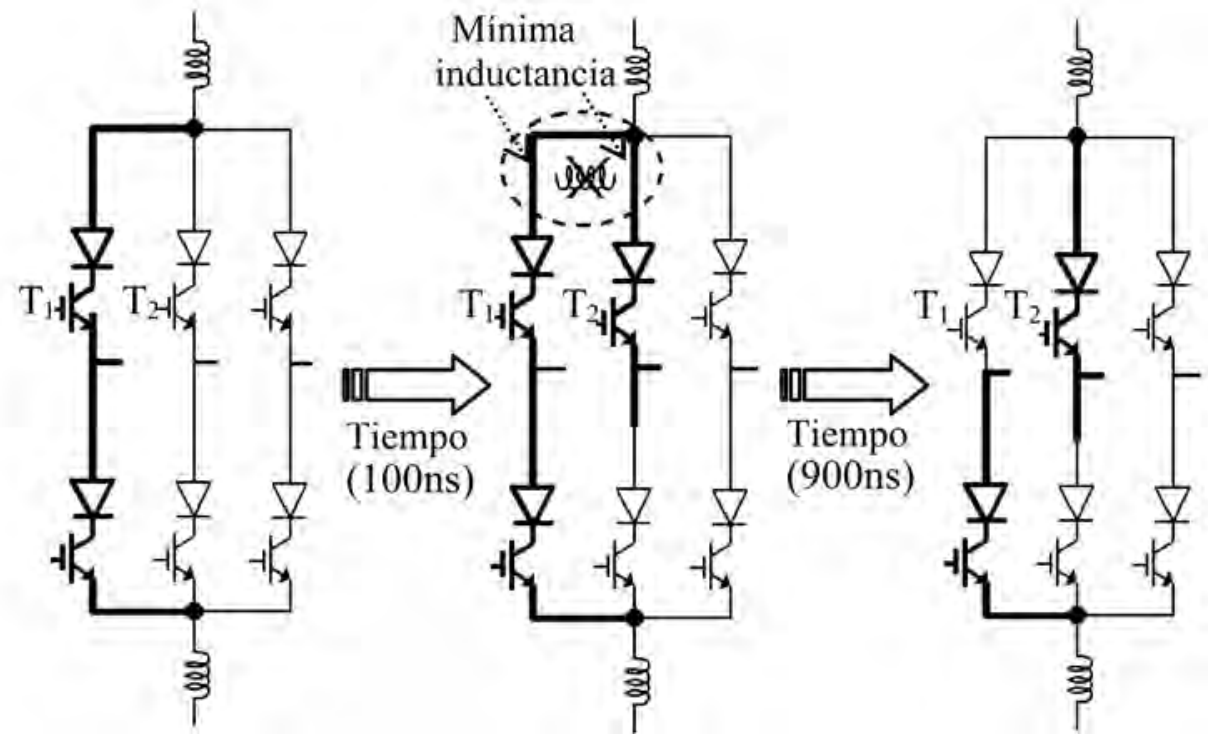

Figura 3.15: conmutación suave debido al solapamiento de tiempos de conducción.

Para que este mecanismo de conmutación funcione correctamente es indispensable minimizar las inductancias parásitas presentes en el camino de la corriente entre llaves, es por ello que la disposición y conexionado (layout) de las llaves se debe diseñar de manera de maximizar el acoplamiento y distribución de corriente en el nodo común entre cada inductor divisor y sus tres llaves correspondientes. De esta manera se consigue minimizar las pérdidas por conmutación en los transistores de las llaves en forma simple y efectiva, sin necesidad de recurrir a complicados algoritmos de modulación como se sugiere en Bai et al. (2011). En el caso particular de esta tesis se realiza el estudio de un MCSI que pueda ser escalable a altas potencias y por ello se utilizarán, tanto en las simulaciones como en la construcción del prototipo experimental, llaves del tipo IGBT, discretas y con circuitos de disparo independientes, como se detalla en el Apéndice A, de manera que el análisis de su funcionamiento pueda ser extrapolado a potencias de varias centenares de KW con mínimas modificaciones. En este tipo de llaves el tiempo de apagado es de aproximadamente $1 \mu s$, mientras que el de encendido puede estar por debajo de los $100 \mathrm{~ns}$, por lo que si se envían en simultáneo dos señales, una de apagado y otra de encendido, se generará una superposición en la conducción de las llaves de aproximadamente 900ns. Este tiempo es suficiente para que la corriente a conmutar entre una llave y otra pueda distribuirse sin producir sobretensiones, lográndose un prototipo experimental con disipación prácticamente nula en las llaves. Lamentablemente la conmutación de los diodos en serie con los IGBT produce pérdidas durante el apagado que son inevitables, ya que los diodos deben bloquear la tensión inversa que la carga pueda presentar, realizando una conmutación "dura" (Mohan et al., 2005). Aunque estas pérdidas son acotadas debido a la falta de sobretensiones y al efecto de distribución de corrientes mencionado, por lo que no afectan considerablemente el rendimiento del convertidor. 


\subsection{Tolerancia a fallas}

Las estructuras tolerantes a fallas en convertidores MVSI presentadas en la literatura se basan principalmente en la combinación de una o más de las siguientes estrategias: (Welchko et al., 2004)

- duplicar una rama del convertidor

- duplicar la cantidad de llaves

- reducir las prestaciones al producirse una falla

- utilizar complejos circuitos con llaves bidireccionales

Si bien duplicar una rama del convertidor provee tolerancia frente a una falla, esta estrategia tiene las siguientes desventajas: muy alto costo, ya que una rama del convertidor implica aproximadamente un $33 \%$ más de costo, sólo en componentes básicos; la necesidad de contar con llaves rápidas para conmutar la corriente de la rama dañada a la rama auxiliar; la imposibilidad de proteger al circuito frente a fallas en los capacitores del bus de continua; se produce un severo desbalance en una fase de la tensión de salida durante el tiempo de conmutación de la rama dañada, pudiendo ser malinterpretado como falla del sistema por alguna protección que esté aguas abajo del convertidor, generando una desconexión indeseada (Ceballos et al., 2006; Chatzakis y Antonidakis, 2008; Son et al., 2012).

Duplicar la cantidad de llaves, colocando interruptores estáticos basados en TRIACS que son muy sensibles a los altos $\frac{d v}{d t}$ presentes en sus bornes, implica el agregado de nuevos puntos de fallas, además del altísimo costo, de casi un $100 \%$ (Dixon et al., 2007).

Otra alternativa es reducir las prestaciones del convertidor cuando se detecta una falla, modificando la modulación para evitar la utilización de la parte fallada. Esta alternativa ha sido estudiada por numerosos autores, entre ellos Li y Xu (2006), Correa et al. (2006), Ma et al. (2007), Grandi et al. (2011) y Shen et al. (2013). Por ejemplo, en caso de fallar una de las llaves superiores en MVSI del tipo NPC o de Capacitores flotantes se puede optar por reducir la amplitud de la tensión de salida para evitar el uso de las llaves dañadas (Kou et al., 2004; Chen et al., 2004; Li y Xu, 2006). También se puede conectar la rama fallada al punto medio de la tensión de entrada, causando un deterioro en las formas de onda de salida, pero permitiendo que el convertidor pueda seguir trabajando, al menos mientras las condiciones de operación de la carga lo permitan (Ceballos et al., 2006, 2007; Mendes et al., 2008).

Utilizar complejos esquemas circuitales, con múltiples llaves bidireccionales a fin de adaptar el circuito para que continue funcionando a pesar de una o más fallas es una alternativa que ha sido ampliamente analizada en la literatura (Hu et al., 2013; Dixon et al., 2007; Cordeiro et al., 2011). Su elevada complejidad requiere una gran potencia de cálculo a fin de considerar todas las combinaciones de conmutación y además mantener el balance de los elementos pasivos (generalmente capacitores). Su costo es relativamente elevado y además, generalmente, los componentes agregados se pueden utilizar únicamente para la adaptación del circuito en caso de falla e implican un costo extra durante el funcionamiento normal sin 
que puedan utilizarse para aumentar el número de niveles o la capacidad de corriente, tensión o potencia del convertidor.

Por otro lado, en la mayoría de las soluciones mencionadas, parte del circuito conectado a las llaves dañadas durante una falla sigue energizado y/o en operación, por lo que es imposible realizar un mantenimiento correctivo sin la desconexión total del convertidor (Chen et al., 2004; Chatzakis y Antonidakis, 2008; Dijkhuizen y Norrga, 2011; Shen et al., 2013).

\subsubsection{Tolerancia a fallas en el MCSI Simétrico}

La construcción totalmente modular es la principal ventaja de la topología MCSI Simétrico y ello permite realizar estructuras tolerantes a fallas (Fault Tolerant) aptas para su operación en ambientes industriales donde la seguridad y confiabilidad deben maximizarse, con la sola inclusión de un módulo extra de respaldo en caliente "Hot Spare". Como se muestra en la Figura 3.16, el módulo de respaldo puede conectarse en paralelo con la carga y directamente al bus de corriente continua $\left(I_{D C}\right)$ en cualquier momento, aún con el convertidor funcionando y de la misma manera puede retirarse un módulo dañado, ya que mientras se mantienen sus llaves apagadas los módulos no consumen corriente, ni afectan al normal funcionamiento del resto del convertidor. Al contrario de lo que sucede en la mayoría de las configuraciones tolerantes a falla de los MVSI, en esta topología los módulos de respaldo también pueden ser utilizados durante el funcionamiento normal del convertidor, por ejemplo para aumentar el número de niveles, para incrementar la frecuencia efectiva del ripple en la salida o durante condiciones especiales de carga y sus llaves tienen los mismos requerimientos de tensión, corriente y $\frac{d v}{d t}$, por lo que no incrementan la probabilidad de fallas del convertidor en conjunto. Para poder recuperar en forma segura la energía almacenada en los inductores

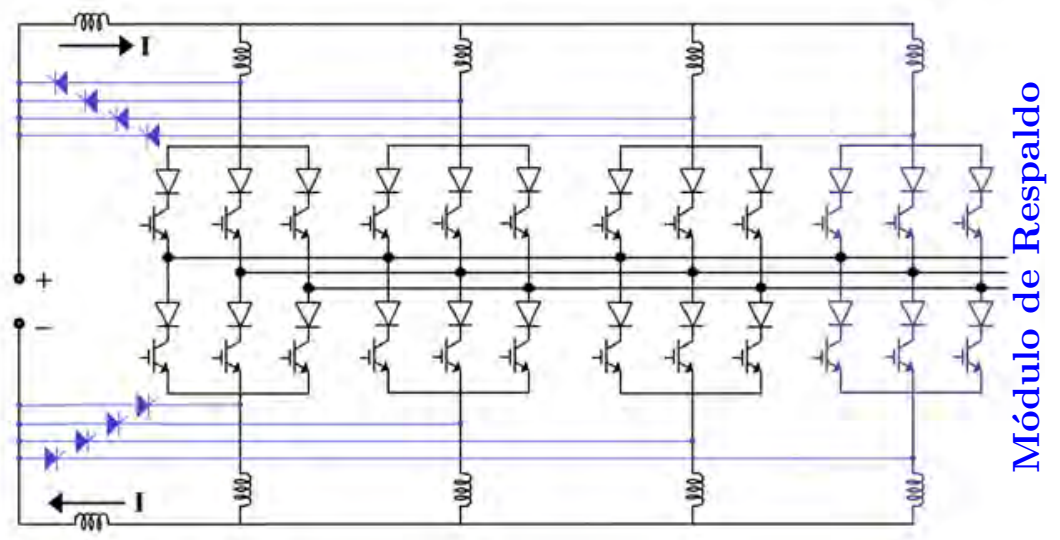

Figura 3.16: diagrama esquemático del arreglo tolerante a fallas.

divisores de un módulo dañado, a fin de permitir su desconexión, es necesario agregar dos llaves unidireccionales por módulo. En la Figura 3.16 se muestra un MCSI Tolerante a Fallas, de siete niveles, con un módulo de respaldo en caliente y sus llaves asociadas.

$\mathrm{Al}$ detectarse una falla en las llaves de algún módulo, de circuito abierto o cortocircuito, se produce la secuencia de eventos que se muestra en las Figuras 3.17, 3.18, 3.19 y 3.20. 


\section{Encendido de llaves auxiliares:}

Cuando se detecta una falla, se accionan las llaves auxiliares (SCR) del módulo dañado, como se muestra en la Figura 3.17. Esto es especialmente importante al detectar una falla por circuito abierto, a fin de garantizar la circulación de la corriente de los inductores divisores del módulo, evitando sobretensiones que puedan dañar a otras llaves u otros componentes del convertidor. Mientras tanto el módulo de reserva continua apagado.

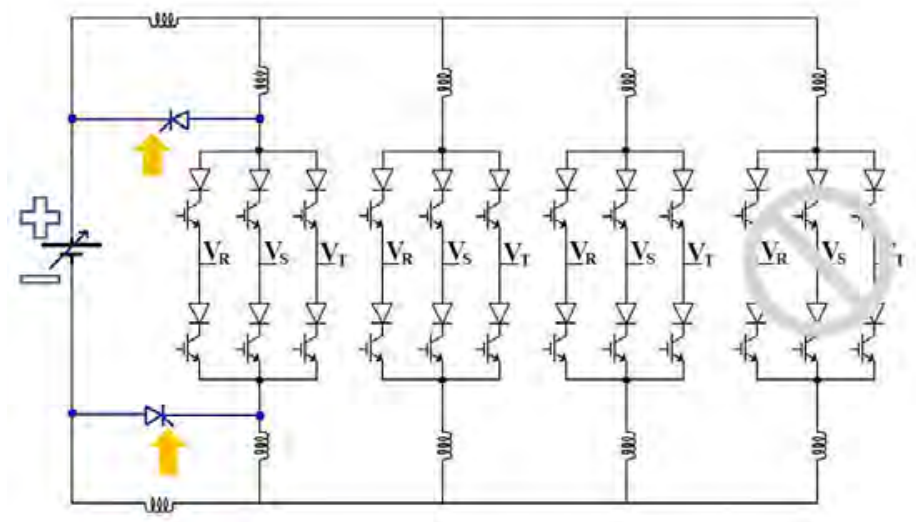

Figura 3.17: secuencia de eventos luego de detectarse una falla en el Módulo 1: se encienden las llaves auxiliares del módulo en falla.

\section{Apagado del módulo dañado:}

Las llaves auxiliares permiten que la corriente de los inductores divisores circule a través de los inductores principales, como se muestra en la Figura 3.18. En este momento se pueden apagar todas las llaves del módulo dañado y proceder a su reemplazo en forma segura, ya que puede ser aislado del resto del convertidor.

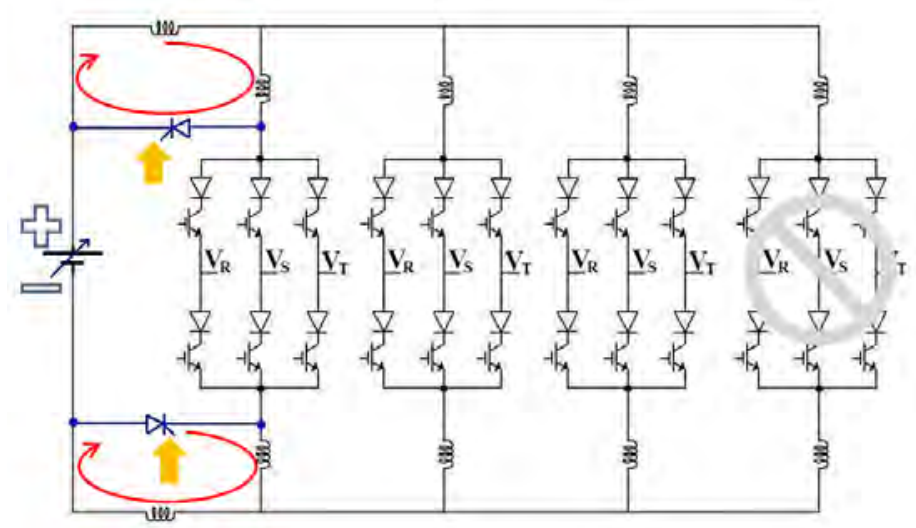

Figura 3.18: secuencia de eventos luego de detectarse una falla en el Módulo 1: se garantiza la circulación de corriente de los inductores del módulo dañado, a través de los inductores principales. 


\section{Descarga de inductores divisores:}

Debido a la energía de las inductancias divisoras, la corriente de las inductancias principales tenderá a subir. Para evitar esto, el controlador PI encargado de regular la corriente principal, Figura 3.19, deberá disminuir la tensión de entrada, forzando una tensión negativa sobre ambas inductancias divisoras, lo que conlleva la reducción de la energía almacenada hasta su extinción.

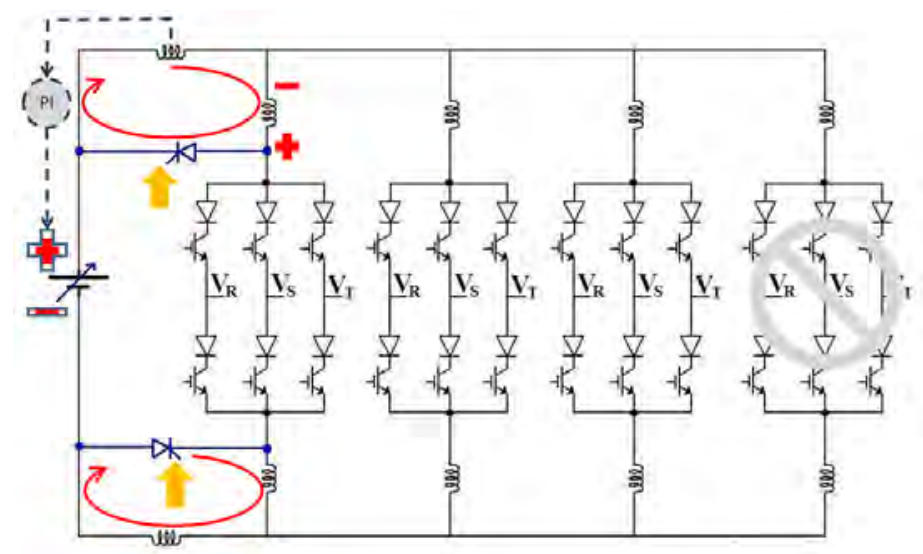

Figura 3.19: secuencia de eventos luego de detectarse una falla en el Módulo 1: el control de corriente disminuye $V_{I N}$ para mantener la corriente principal.

\section{Encendido de módulo de respaldo:}

Una vez que el sistema de supervisión del MCSI verifica que los pasos anteriores han sido correctamente cumplidos y que se garantiza la seguridad del convertidor a pesar de la falla, se puede reenviar las señales de control de las llaves del módulo dañado a las llaves del módulo de reserva, el que comenzará a cargar sus inductores divisores, como se muestra en la Figura 3.20. El ritmo de carga de los inductores divisores depende de la ecuación básica:

$$
\frac{d i}{d t}=\frac{V}{L}
$$

donde $V$ es la tensión sobre el inductor, que en este caso es similar a la de alimentación, aunque también depende en menor medida del estado de carga del convertidor. Dado que la suma de las corrientes internas debe ser igual a la corriente principal, el ritmo de carga de los inductores del nuevo módulo es idéntico al de descarga de los inductores del módulo dañado, permitiendo evitar sobretensiones y minimizando la distorsión a la salida durante el proceso de puesta en marcha del módulo de respaldo.

El tiempo requerido para estas cuatro etapas de la protección de fallas está limitado por la velocidad de cálculo del sistema supervisor y por la selectividad del sistema de detección de fallas, además dado que no es necesario realizar cálculos complejos y se pueden utilizar llaves auxiliares SCR rápidos, es posible realizar toda la secuencia de puesta en marcha del módulo 


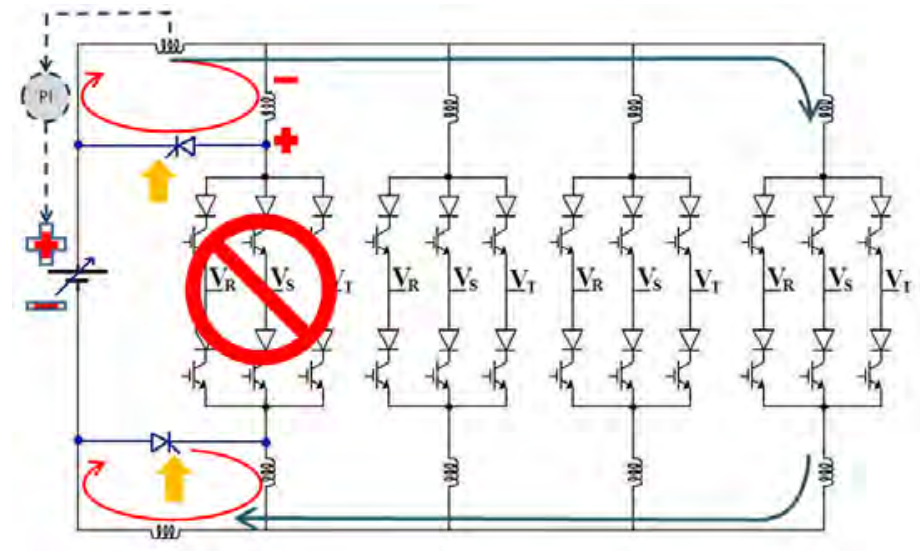

Figura 3.20: secuencia de eventos luego de detectarse una falla en el Módulo 1: se traslada el control al módulo de respaldo y el convertidor vuelve a quedar completamente operativo.

de respaldo en sólo algunas centenas de microsegundos. Por otro lado, el módulo de reserva comenzará a entregar su corriente a la carga ni bien se conecta, minimizando el tiempo de falla del convertidor. Al agotarse la energía de los inductores divisores del módulo dañado, las llaves auxiliares se apagarán naturalmente por lo que el módulo dañado puede removerse para su reparación y/o reemplazo por uno nuevo. La secuencia de puesta en marcha del módulo de reserva, y por lo tanto de desconexión de un módulo en servicio, puede realizarse en cualquier momento, permitiendo la realización de reparaciones, ciclado de módulos y/o mantenimiento preventivo sin necesidad de un apagado completo del convertidor, aumentando enormemente su vida útil y minimizando la probabilidad de fallas por envejecimiento de componentes. Esta estrategia permite tolerancia a fallas, no sólo de las llaves, sino también de los inductores divisores, maximizando el MTBF y por lo tanto la seguridad de operación de todo el convertidor. La construcción totalmente modular de esta topología permite además el agregado de módulos extra en caso que sea necesario aumentar el nivel de tolerancia a fallas. Cabe destacar que la carga no queda sin alimentación en ningún momento de la secuencia de reemplazo de módulos, aunque sí pueden producirse perturbaciones durante el tiempo que tardan en cargarse a su valor nominal los inductores del módulo de reserva.

En la Figura 3.21 pueden observarse los resultados de simulación de la secuencia descripta (Aguirre et al., 2010b). La corriente de la fuente disminuye durante el instante de conmutación porque las inductancias divisoras aportan su energía a las inductancias principales. La corriente de las inductancias divisoras del módulo dañado se extinguen en aproximadamente $30 \mathrm{~ms}$. La corriente de la carga sólo sufre una pequeña perturbación que dura mucho menos que un ciclo y por lo tanto no producirá efectos graves, ni en los sistemas alimentados por el MCSI, ni en sus protecciones. 

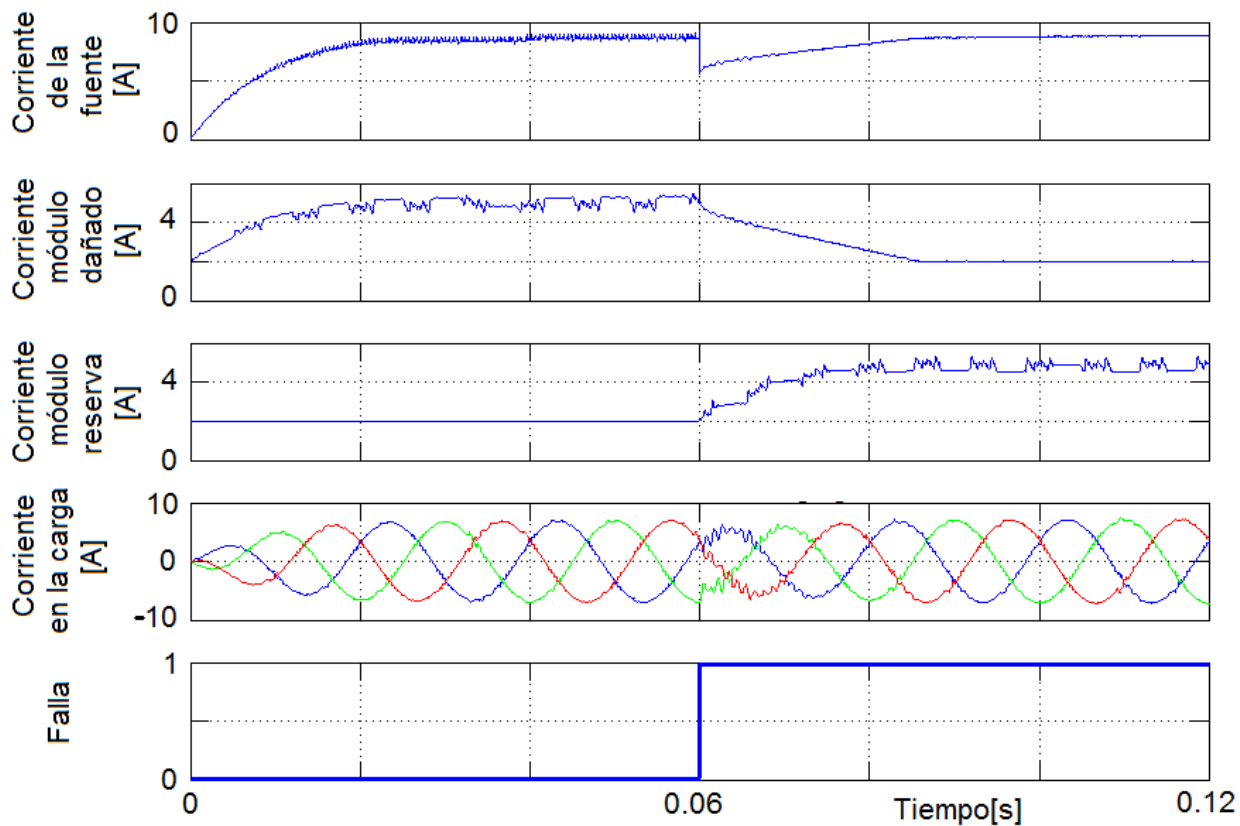

Figura 3.21: simulación de la secuencia de eventos luego de detectada una falla.

\subsection{Balance de corrientes}

El problema del balance de los capacitores en las distintas topologías MVSI (enclavamiento por diodos, capacitores flotantes e híbridos, entre otros) ha sido ampliamente tratado en la literatura (Peng, 2001; Rech y Pinheiro, 2007; Verne y Valla, 2010a,b; Ewanchuk et al., 2011), lo que no ocurre con el balance de corrientes en los inductores de los MCSI.

La modulación con desfasaje de portadoras (PSC-SPWM) produce un balance natural de las corrientes en los inductores divisores, en forma similar al balance de capacitores en un MVSI de capacitores flotantes. En la Figura 3.22 se muestran las corrientes I1, I2 e I3, donde puede observarse que las corrientes de los inductores divisores presenta un ripple característico causado por la modulación, formado por una serie de oscilaciones y una zona constante. La zona constante se obtiene en los intervalos en los cuales alguna de las corrientes de carga está pasando por su nivel mínimo o máximo, lo cual ocurre tres veces por cada ciclo de la fundamental. Si bien las corrientes mantienen un balance, aún durante el transitorio de arranque, el valor medio de cada corriente no es exactamente igual al de las otras, como puede observarse en los detalles de la Figura 3.22. Esto es debido a que la parte constante de la corriente tiene un valor distinto para cada módulo, el que depende de la fase relativa entre la portadora (triangular) del módulo y las sinusoides de referencia.

Los métodos de control de balance utilizados en los convertidores MVSI implican la necesidad de realizar complejas mediciones y requieren una enorme potencia de cálculo en tiempo real (McGrath y Holmes, 2008b). Esta situación se complica aún más en el caso de la topología MCSI utilizada, debido a que las tres fases de la salida están acopladas y por lo tanto todo el convertidor es un sistema completamente acoplado. Además, la topología 


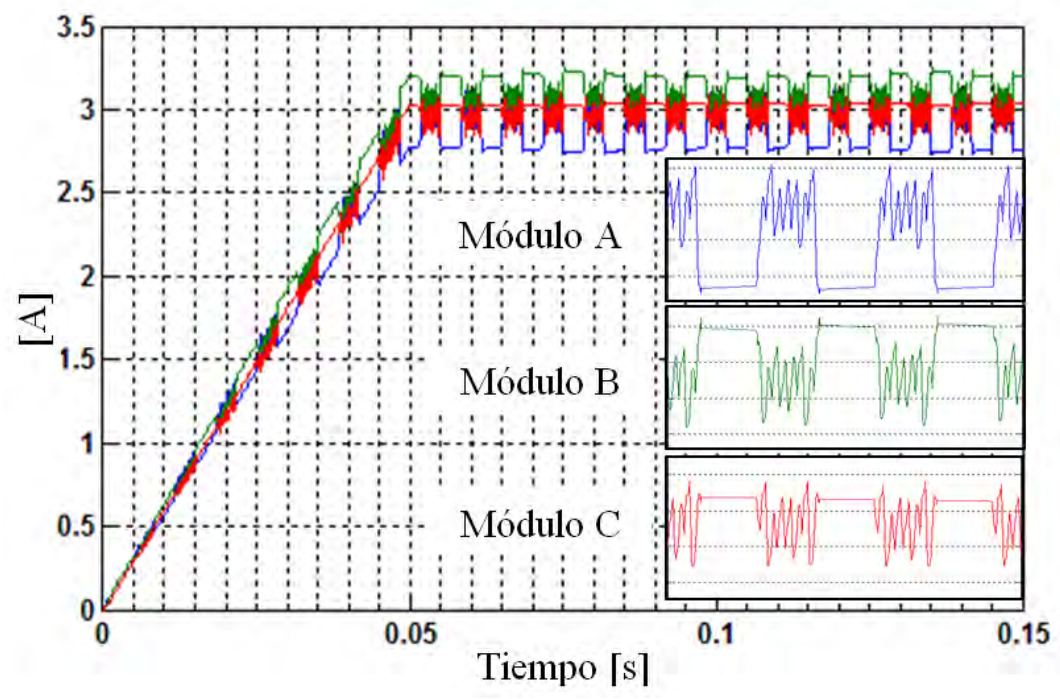

Figura 3.22: balance natural de corrientes mediante modulación PSC-SPWM $\left(m_{a}=0,5\right)$.

utilizada no es el dual de ninguno de los convertidores multinivel de tensión, por lo que no es posible utilizar las técnicas de balance y control desarrolladas para los MVSI y que se encuentran en la literatura. Por ello se descarta realizar el balance forzado de las corrientes internas del convertidor mediante el uso de la modulación vectorial, tal como sugieren Xiong et al. (2004b) y Zmood y Holmes (1998).

Haciendo uso de la capacidad propia de la modulación PSC-SPWM, se busca un método alternativo para forzar el balance de las corrientes frente a desbalances producidos, tanto por el funcionamiento normal de la modulación PSC-SPWM (error en estado estacionario), como por perturbaciones entre las que se encuentran: variaciones o diferencias en los valores reales de los componentes, errores de cuantificación y muestreo del control, tiempos de conmutación de los transistores, etc.. Dado que la posición de la zona plana de las corrientes depende, para cada módulo, de la relación entre su portadora triangular y las señales sinusoidales de referencia, se encontró que es posible alterar el valor de esa parte del ripple de corriente mediante pequeñas modificaciones en las triangulares de la modulación. Realizando un análisis de la modulación y sus efectos en las corrientes internas del convertidor, se detectaron dos métodos posibles para modificar el valor medio deseado:

- Desplazamiento de fase de portadora : se modifica la fase relativa de una portadora con respecto a las otras dos, atrasándola o adelantándola con respecto al valor nominal de $120^{\circ}$ (Figura 3.23(a)).

- Variación de amplitud de portadora : se modifica la amplitud de una portadora con respecto a las otras dos, amplificándola o atenuándola con respecto al valor unitario de referencia (Figura 3.23(b)). 


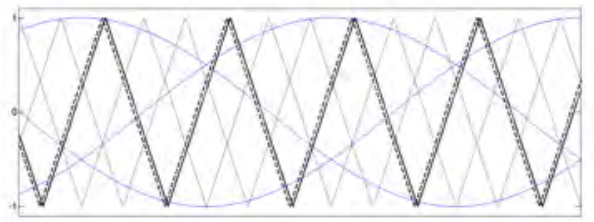

(a) variación de la fase relativa de una portadora.

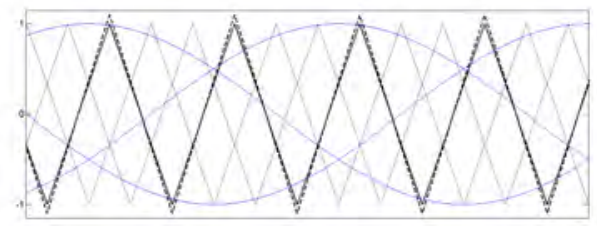

(b) variación de la amplitud de una portadora.

Figura 3.23: principio básico de los métodos de balance de corrientes internas.

\subsubsection{Control por desplazamiento de fase}

Si bien la modulación PSC-SPWM requiere que la diferencia de fase entre las portadoras sea de $120^{\circ}$ (para un convertidor de siete niveles y 3 módulos), es posible cambiar ligeramente ese desfasaje para modificar el ripple de tensión en las inductancias divisoras y por consiguiente el valor medio de corriente en ellas, sin alterar el funcionamiento general del convertidor. Este método de control requiere mínima capacidad de cálculo y puede ser implementado fácilmente en la misma FPGA en la que se programa la modulación. Alterando la fase relativa entre portadoras se modifica el punto en el cual se estabiliza la zona constante del ripple de la corriente de los inductores divisores. De esta manera se puede alterar ligeramente el valor medio de las corrientes, cuyo efecto integrador permite mantener las mismas en el valor deseado, consiguiendo un balance con mínimo error en estado estacionario.

\section{Identificación del sistema}

A fin de diseñar una estrategia de control adecuada, es preciso primero realizar la identificación del sistema cuya entrada es la señal de control, fase relativa de una portadora, y su salida es el valor medio de las corrientes de los inductores divisores. Para obtener el valor medio de las corrientes se utiliza un filtro pasa-bajos para eliminar las componentes de conmutación y luego se toma el promedio entre las corrientes de los dos inductores de cada módulo. De esta manera se obtiene una señal representativa de las corrientes de cada módulo para analizar la respuesta de estas señales frente a pequeñas perturbaciones en la fase de las portadoras.

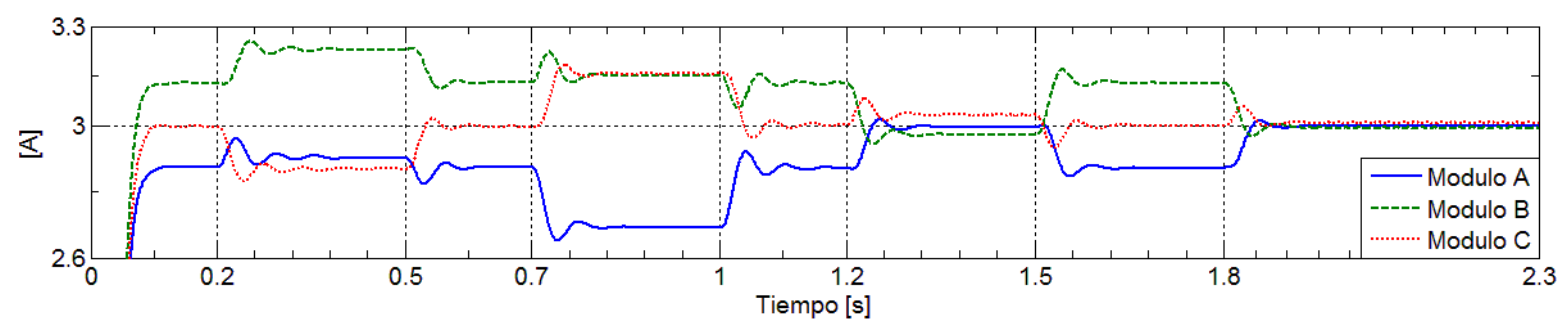

Figura 3.24: variaciones de las corrientes de cada módulo, debidas a cambios en las fases de portadora. 
En la Figura 3.24 puede observarse la respuesta del sistema frente a distintas variaciones en las fases de las portadoras, observando el valor medio de las corrientes del convertidor. Cada traza representa la corriente media por módulo, por ejemplo para el módulo $A$ se pasan las corrientes $I 1$ e $I 4$ (definidas en la Figura 3.2) por un filtro pasa-bajos y luego se promedian. El convertidor arranca a lazo abierto y sin control, estabilizándose en los valores determinados por la modulación PSC-SPWM, donde se aprecia el error en estado estacionario mencionado anteriormente.

- A los 0,2 segundos se adelanta la fase de la portadora $A$ un $1 \%$. Se puede apreciar que la corriente media del módulo $A$ se mantiene prácticamente constante, mientras que la del módulo $B$ aumenta y la del módulo $C$ disminuye considerablemente.

- A los 0,5 segundos se elimina el desfasaje aplicado y el sistema vuelve al punto de equilibrio dictado por la modulación.

- A los 0,7 segundos se adelanta la fase de la portadora $B$ un $1 \%$. Puede observarse que la corriente del módulo $B$ se mantiene aproximadamente constante, mientras que la del módulo $C$ aumenta y la del módulo $A$ disminuye.

- A los 1,0 segundos se elimina el desfasaje aplicado y el sistema vuelve al punto de equilibrio dictado por la modulación.

- A los 1,2 segundos se adelanta la fase de la portadora $C$ un $1 \%$. Puede observarse que la corriente del módulo $C$ se mantiene relativamente constante, mientras que la del módulo $A$ aumenta y la del módulo $B$ disminuye.

- Luego de alcanzado nuevamente el equilibrio, a los 1,8 segundos se muestra como se puede reducir ampliamente el desbalance aplicando los siguientes desfasajes a cada una de las portadoras: Fase $A=0 \%$, FaseB $=-0,3 \%$, FaseC $=0,9 \%$

Debe notarse que la suma de las corrientes de los tres módulos debe ser siempre igual a la corriente de la fuente principal, por lo tanto las variaciones de las corrientes se producen en forma simétrica. En la Figura 3.25(a) puede observarse en detalle la reacción de la corriente media de cada módulo frente a modificaciones de la fase de la portadora $B$. Luego de que el sistema se estabilice, a los 0,2 segundos, se aplica una corrección de fase de $+1 \%$. Mientras que a los 0,5 segundos se modifica el cambio de fase a $-1 \%$. En ambos casos, una vez pasado el transitorio, el efecto sobre la corriente media del módulo $B$ es casi nulo, mientras que la corriente media del módulo $A$ se modifica en sentido inverso al cambio de fase aplicado y la del módulo $C$ lo hace en el mismo sentido que el cambio de fase.

En la Figura 3.25(b) se puede apreciar en detalle la reacción de la corriente media de cada módulo frente a modificaciones de la fase de la portadora $C$. Luego de que el sistema se estabilice, a los 0,2 segundos, se aplica una corrección de fase de $+1 \%$. A los 0,5 segundos se modifica el cambio de fase a $-1 \%$. Una vez pasado el transitorio el efecto sobre la corriente del módulo $C$ es casi nulo, mientras que la corriente del módulo $A$ se modifica en el mismo sentido que el cambio de fase aplicado y la del módulo $B$ en el sentido inverso al cambio de fase. 


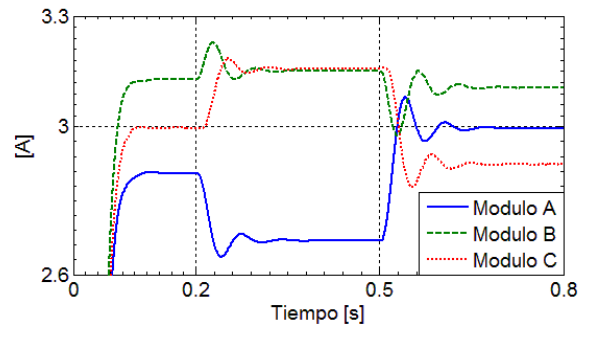

(a) cambio de fase de la portadora $B$.

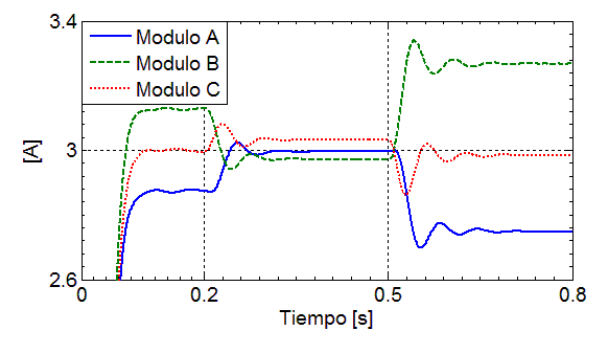

(b) cambio de fase de la portadora $C$.

Figura 3.25: detalle de los efectos provocados al cambiar la fase de las portadoras, $+1 \%$ a los $0,2 s$ y $-1 \%$ a los $0,5 s$.

Las observaciones de las Figuras 3.24 y 3.25 se resumen en el Cuadro 3.6. Cabe destacar, a partir del análisis realizado, que una variación en la fase de la portadora del módulo $B$ provoca la reducción del error entre la corriente media del módulo $C$ y el $A$, modificando levemente el valor de corriente del módulo $B$. Asimismo, una variación en la fase de la portadora del módulo $C$ genera la reducción del error entre la corriente media del módulo $A$ y el $B$, dejando prácticamente constante el valor de la corriente media en el módulo $C$. A raíz de esto, ambas variaciones pueden ser utilizadas de forma simultánea para controlar las corrientes ya que se afectan levemente entre ellas. La ganancia del sistema ante una perturbación de fase es de aproximadamente $0,125 \mathrm{~A} /{ }^{\circ} \mathrm{D}$, donde ${ }^{\circ} \mathrm{D}$ es el desfasaje (en grados) aplicado a la fase de la portadora seleccionada.

Cuadro 3.6: resumen de las variaciones en las corrientes internas del convertidor provocadas por cambios de fase en las portadoras.

\begin{tabular}{lccc}
\hline Cambio & MóduloA & Módulo B & Módulo C \\
de fase & & & \\
\hline $\mathrm{A}+$ (adelanto) & $\approx$ & $\Uparrow$ & $\Downarrow$ \\
$\mathrm{A}-($ atraso $)$ & $\approx$ & $\Downarrow$ & $\Uparrow$ \\
$\mathrm{B}+($ adelanto $)$ & $\Downarrow$ & $\approx$ & $\Uparrow$ \\
$\mathrm{B}-($ atraso $)$ & $\Uparrow$ & $\approx$ & $\Downarrow$ \\
$\mathrm{C}+$ (adelanto $)$ & $\Uparrow$ & $\Downarrow$ & $\approx$ \\
$\mathrm{C}-($ atraso $)$ & $\Downarrow$ & $\Uparrow$ & $\approx$ \\
\hline
\end{tabular}

\section{Control por cambio de fase a lazo cerrado}

Con los datos obtenidos de la identificación del sistema es posible diseñar un control a lazo cerrado para supervisar y garantizar el balance de las corrientes internas, teniendo en cuenta que la dinámica del sistema estará dominada por la respuesta de los filtros de valor medio, ésta puede hacerse tan lenta como el sistema lo permita, para minimizar el efecto del ripple en el valor medio de las señales. El control a lazo cerrado se implementa mediante dos 
controladores de acción proporcional e Integral (PI) como se muestra en la Figura 3.26. Para obtener el valor medio de las corrientes se aplica un filtro pasa-bajos de segundo orden, luego se promedian las corrientes de cada módulo ( $I 1$ e $I 4, I 2$ e $I 5$ y I3 e I6) y luego, teniendo en cuenta los resultados mostrados en el Cuadro 3.6, se obtienen las señales de error mediante la resta entre las corrientes de los módulos $A$ y $C$ (para actuar sobre la portadora $B$ ) y de los módulos $B$ y $C$ (para actuar sobre la portadora $A$ ). La dinámica resultante que se observa en las figuras de este análisis es la composición del ripple del sistema con la respuesta del filtro. Dado que el propósito de este análisis es validar el método de control, sin perder generalidad mediante la adopción de un filtro óptimo, se utiliza un simple pasa-bajos de segundo orden, pero con frecuencia de corte restrictiva $(15 \mathrm{~Hz})$ a fin de eliminar las componentes de ripple. Por otro lado, se busca sintonizar los controladores PI para obtener a lazo cerrado una respuesta rápida pero con poco sobrepico y buen factor de amortiguamiento, que altere lo menos posible la salida trifásica de corriente del convertidor.

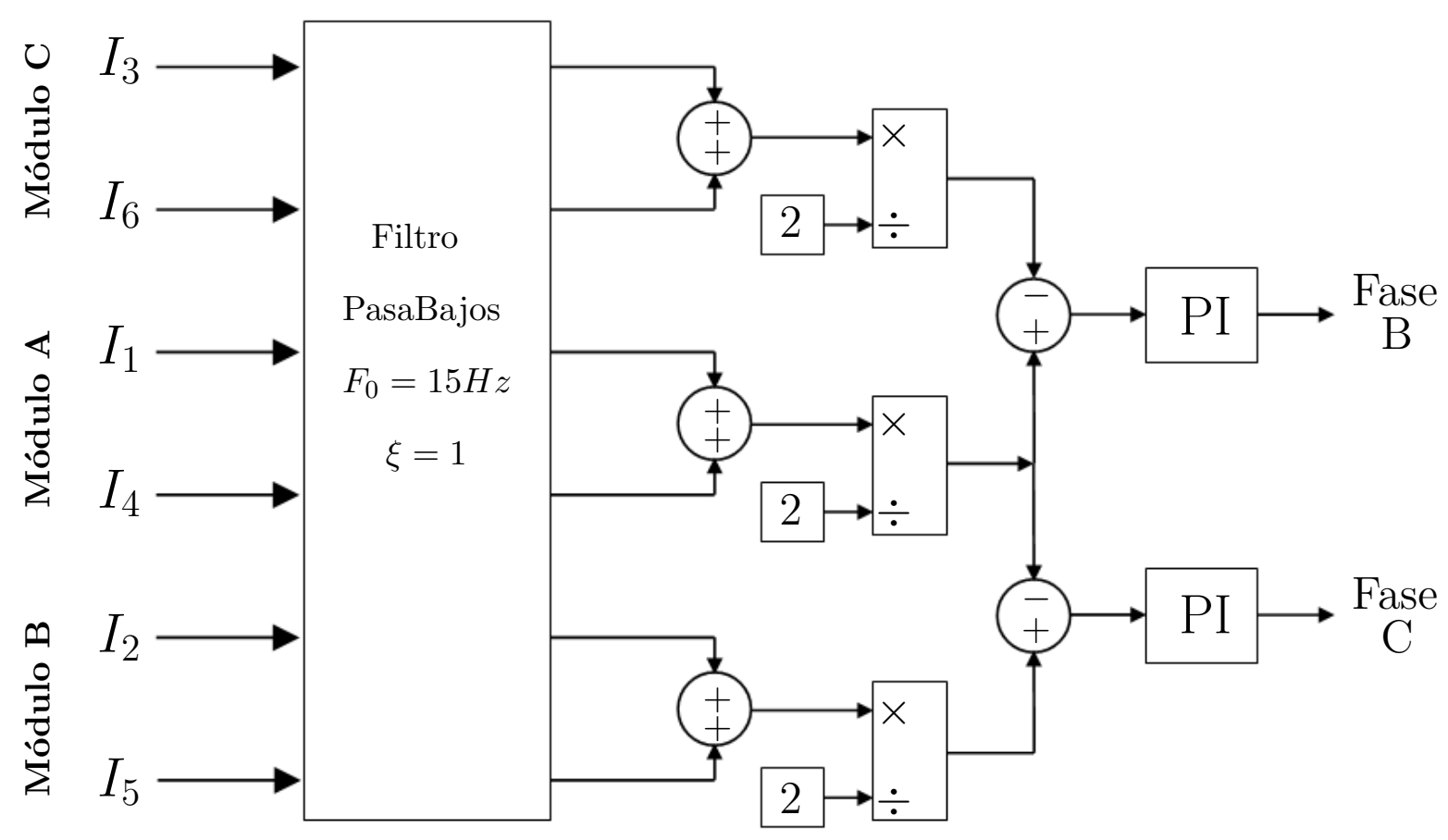

Figura 3.26: esquema de la estructura de control por variación de fase de portadoras.

En la Figura 3.27 se puede observar el arranque del convertidor con el control por cambio de fase activado. Se observa que las corrientes se estabilizan en aproximadamente $0,3 s$ con un error prácticamente nulo. A los $0,4 s$ se desactiva el control y por lo tanto el convertidor vuelve a la situación de balance natural, con error en estado estacionario. A los $0,7 \mathrm{~s}$ se vuelve a activar el control, produciendo, luego del transitorio, un balance casi perfecto de las corrientes. Esta simulación se realizó utilizando un $m_{a}=0,35$ y se observa una muy buena respuesta dinámica del conjunto a lazo cerrado. La modulación PSC-SPWM produce una compensación de las portadoras de cada módulo de manera que en el espectro de la corriente 
de salida sólo debería aparecer la resultante equivalente a la frecuencia de portadora de un módulo multiplicada por el número de módulos del convertidor $(n * f s w)$. En la práctica, los diversos errores de muestreo (cuantificación, discretización, tiempos de conmutación, etc.) y las asimetrías del circuito evitan que la compensación teórica de portadoras se produzca completamente, apareciendo componentes adicionales en el espectro. Si bien el método de control propuesto produce un deterioro en las condiciones de compensación de la modulación al modificar las fases relativas de las portadoras, el porcentaje de desfasaje necesario para alcanzar el balance de corrientes es tan pequeño que las perturbaciones en el espectro de las corrientes de salida del convertidor son completamente despreciables, tal como se muestra en la Figura 3.28.

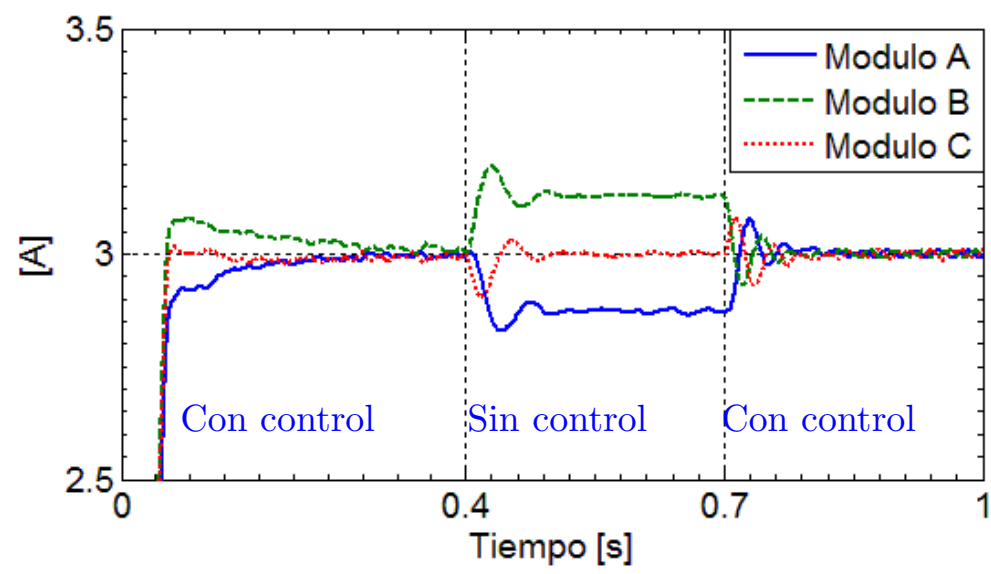

Figura 3.27: efecto del control a lazo cerrado mediante cambios en las fases relativas de portadora.

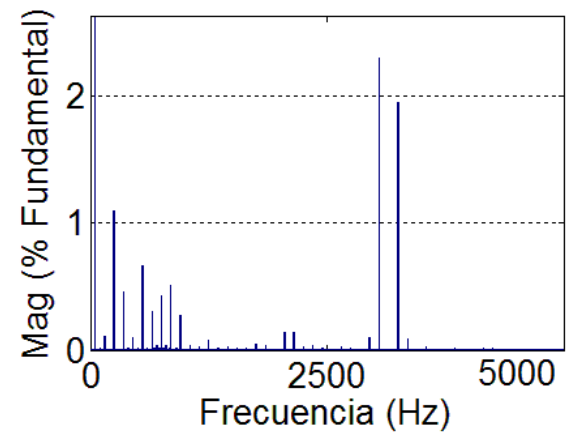

(a) Sin control: $T H D=3,44 \%$, Fundamental $=2,624 A$

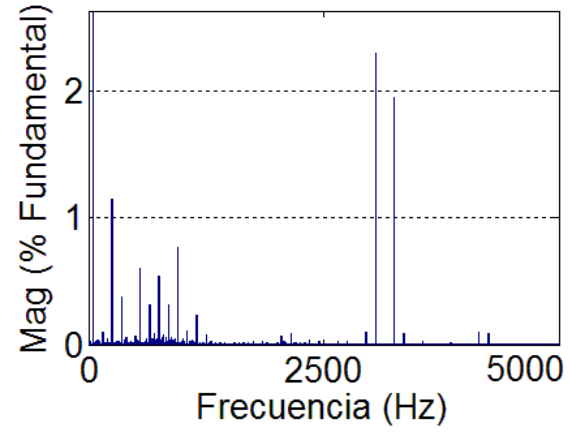

(b) Con control: $T H D=3,5 \%$, Fundamental $=2,628 \mathrm{~A}$.

Figura 3.28: espectros de la corriente de salida $\left(m_{a}=0,35\right)$ : (a) con la modulación PSC-SPWM original, (b) con los efectos del control por cambio de fase de portadoras. 


\subsubsection{Control por amplitud de portadora}

En forma análoga al control por cambio de fase, se detectó una marcada dependencia entre la amplitud de las portadoras triangulares y el valor medio de las corrientes de los módulos, con la diferencia de que es posible modificar la amplitud de una portadora sin modificar las otras, por lo tanto se dispone de tres entradas de control. Lamentablemente, se sigue teniendo un sistema acoplado, ya que modificar la amplitud de una portadora afectará a las corrientes de dos módulos, debido a que el ripple de corriente de los inductores de cada módulo está determinado por la conmutación de los otros dos, complicando el control. Por otro lado, la modificación de la amplitud de cada portadora debe ser mínima a fin de no afectar el normal funcionamiento de la modulación. El efecto de este control se hace más efectivo cuando el punto de trabajo del convertidor es alto $\left(m_{a}>0,6\right)$, debido a que se obtiene mayor diferencia en el pulso PWM resultante. Como se muestra en la Figura 3.29, cuando la señal de referencia tiene poca amplitud la diferencia entre el pulso con y sin control es mínima (Figura 3.29(a)), mientras que una gran amplitud de la referencia producirá un cambio mucho mayor en el ancho del pulso PWM frente al mismo cambio en la portadora triangular (Figura 3.29(b)).

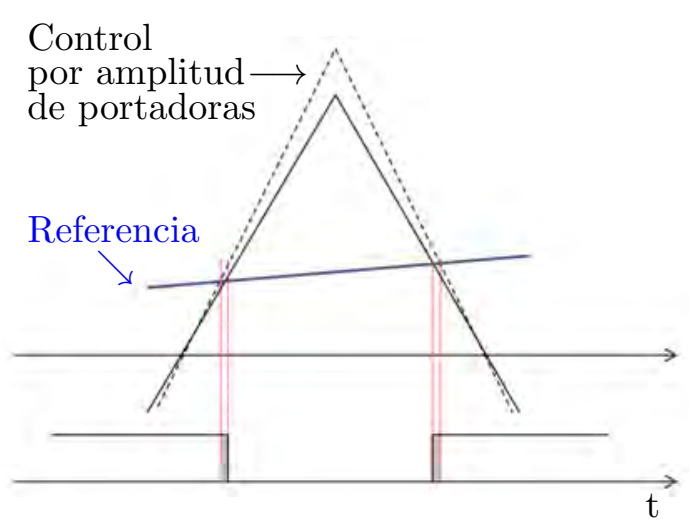

(a) $m_{a}<0,6$

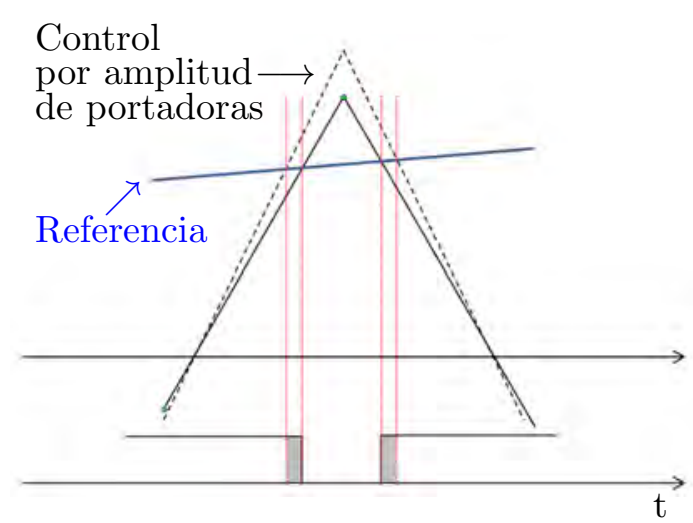

(b) $m_{a}>0,6$

Figura 3.29: efecto del control por cambio de amplitud para distintos puntos de trabajo.

\section{Identificación del sistema}

En la Figura 3.30 puede observarse el comportamiento del sistema frente a cambios en las amplitudes de las portadoras, una a la vez, en forma análoga a la Figura 3.24.

- Hasta los 0,2 segundos el sistema arranca normalmente, mostrando el error en estado estacionario.

- Entre los 0,2 y los 0,5 segundos se aplica un aumento de $2 \%$ a la amplitud de la portadora $C$. Se puede apreciar que la corriente del módulo $C$ permanece prácticamente constante mientras que la del módulo $A$ aumenta y la del $B$ disminuye considerablemente. 


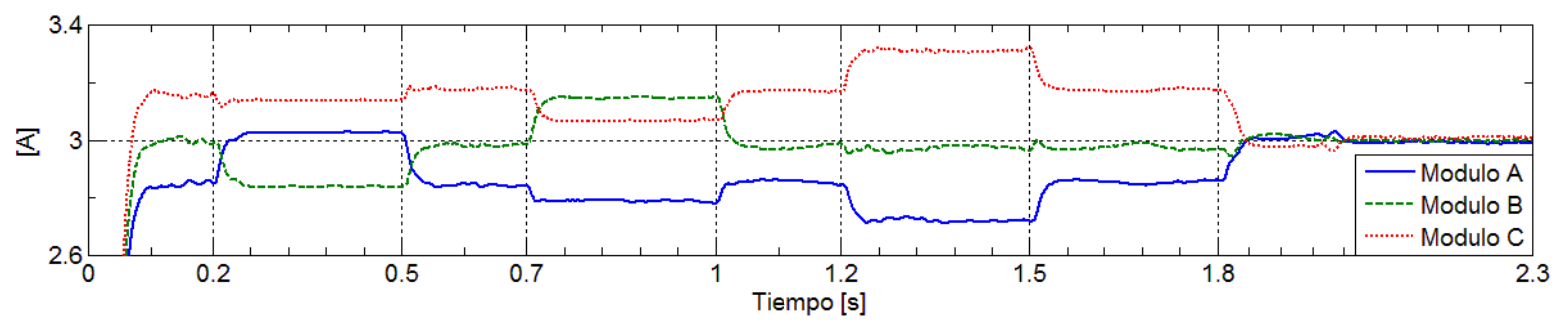

Figura 3.30: variaciones en las corrientes de cada módulo, provocadas por cambios en las amplitudes de portadora.

- Entre los 0,7 y 1 segundo se aplica un aumento de $2 \%$ a la amplitud de la portadora $A$. Se puede observar que la corriente del módulo $A$ permanece prácticamente constante, mientras que la del módulo $B$ aumenta y la del $C$ disminuye notoriamente.

- Entre los 1,2 y 1,5 segundos se aplica un aumento de $2 \%$ a la amplitud de la portadora $B$. Este cambio provoca un aumento de la corriente del módulo $C$ y la disminución de la del módulo $A$ de forma considerable, dejando prácticamente constante la del módulo $B$.

- Finalmente, a los 1,8 segundos se aplican las siguientes correcciones: Fase $A=+2,5 \%$, Fase $B=+2 \%$, FaseC $=-1,5 \%$, demostrándose un ejemplo de valores que garantiza el balance total de las corrientes.

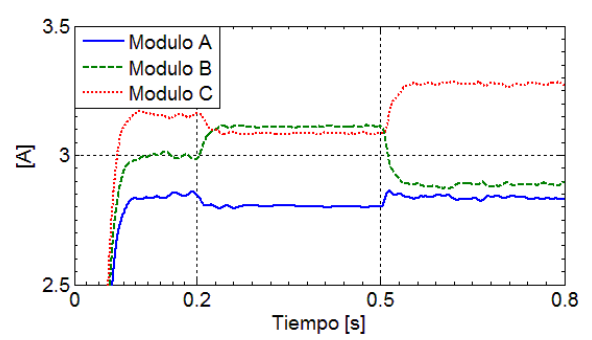

(a) Portadora $A$.

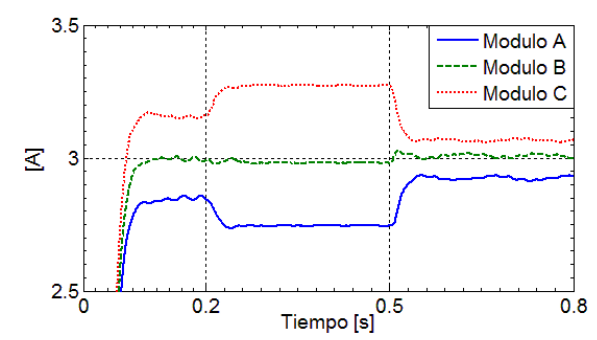

(b) Portadora $B$.

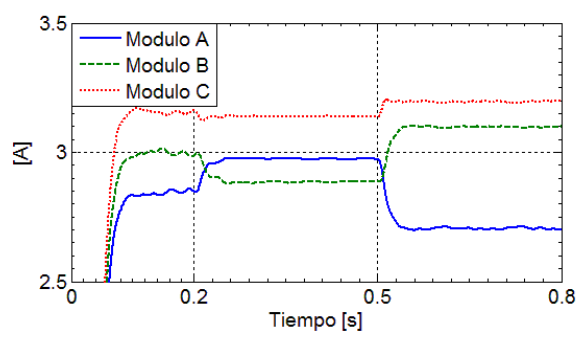

(c) Portadora $C$.

Figura 3.31: detalle de los efectos provocados al cambiar la amplitud de las portadoras, $+2 \%$ a los $0,2 s$ y $-2 \%$ a los $0,5 s$.

En la Figura 3.31 se muestran tres simulaciones donde, una vez que se estabiliza el sistema, a los $0,2 s$ se aumenta un $2 \%$ la amplitud de la portadora de un módulo, mientras que a los 
$0,5 s$ se disminuye esa amplitud en el mismo porcentaje. En la Figura 3.31(a) se observa la modificación de la portadora del módulo $A$. Tanto para el aumento de su amplitud como para su disminución, la corriente media del módulo $A$ no presenta variación notable, sin embargo la corriente media del módulo $B$ se modifica en el mismo sentido que el cambio de amplitud y la del $C$ en el sentido inverso. En la Figura 3.31(b) se observa que ante la variación de la amplitud en la portadora del módulo $B$, la corriente media en el módulo $B$ no presenta cambios notables, mientras que la del $C$ responde en el mismo sentido que el cambio de amplitud y la del $A$ en el sentido inverso. En la Figura 3.31(c), puede apreciarse que ante una variación en la amplitud de la portadora del módulo $C$, la corriente media del módulo $C$ permanece prácticamente constante, la del módulo $A$ se modifica en el mismo sentido que el cambio de amplitud aplicado y la del $B$ en sentido inverso. El efecto del cambio de amplitud de una portadora se propaga a los otros módulos del convertidor debido a que la tensión de ripple en cada inductor, que determina su valor de corriente, está relacionado con el estado de las llaves de los otros módulos del convertidor, además de la tensión de la carga.

Analizando la variación de las corrientes en función del cambio de amplitud en cada portadora, detalladas en las Figuras 3.30 y 3.31, se construye el Cuadro 3.7, donde se puede observar que corrientes son modificadas por cada cambio de amplitud y con que sentido. La ganancia del control resultante es de $50 \mathrm{~mA}$ por cada $1 \%$ de cambio en la amplitud de la portadora seleccionada. Cabe destacar que se puede utilizar como acción de control a la amplitud de cada una de las portadoras en forma simultánea, considerando el acoplamiento descripto.

Cuadro 3.7: resumen de las variaciones en las corrientes internas del convertidor provocadas por cambios de amplitud en las portadoras.

\begin{tabular}{cccc}
\hline $\begin{array}{c}\text { Cambio } \\
\text { amplitud }\end{array}$ & MóduloA & Módulo B & Módulo C \\
\hline $\mathrm{A}+$ & $\approx$ & $\Uparrow$ & $\Downarrow$ \\
$\mathrm{A}-$ & $\approx$ & $\Downarrow$ & $\Uparrow$ \\
$\mathrm{B}+$ & $\Downarrow$ & $\approx$ & $\Uparrow$ \\
$\mathrm{B}-$ & $\Uparrow$ & $\approx$ & $\Downarrow$ \\
$\mathrm{C}+$ & $\Uparrow$ & $\Downarrow$ & $\approx$ \\
$\mathrm{C}-$ & $\Downarrow$ & $\Uparrow$ & $\approx$ \\
\hline
\end{tabular}

\section{Control por amplitud a lazo cerrado}

En la Figura 3.32 se muestra la estructura de control utilizada para el balance por variación de amplitud de portadoras. Se utiliza una estrategia similar a la presentada para el control por fase, pero utilizando tres controladores PI a fin de distribuir la acción de control entre las tres portadoras y así obtener una mínima deformación de la modulación original, limitando la excursión de salida de los controladores a sólo $\pm 3 \%$. En este caso, se utilizó un filtro 
pasa-bajos con frecuencia de corte en $20 \mathrm{~Hz}$ y mayor amortiguamiento, para tener menores sobrepicos y oscilaciones que en el caso del control por fase. Las señales de error se obtienen de la resta de las corrientes promedio de los módulos $B$ y $C$ para accionar sobre la amplitud de la portadora del módulo $A$, la resta de $C$ y $A$ para accionar sobre la portadora del módulo $B$ y la resta de $A$ y $B$ para accionar sobre la portadora del módulo $C$.

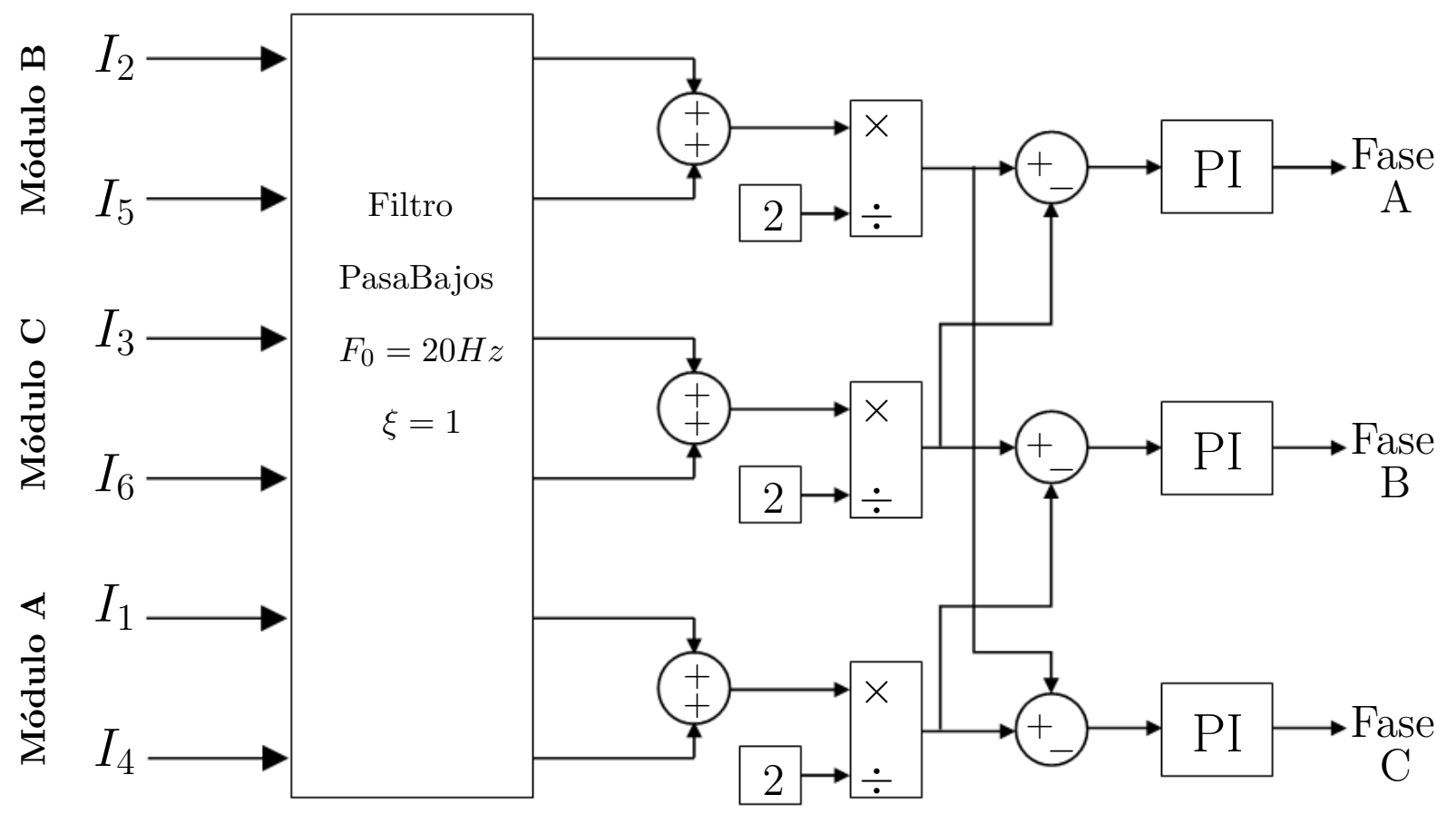

Figura 3.32: esquema de la estructura de control por variación de amplitud de portadoras.

En la Figura 3.33 se puede observar el arranque del convertidor con el control por cambio de amplitud a lazo cerrado activado, lo que produce un balance en las corrientes con un error prácticamente nulo. A los $0,4 s$ se desactiva el control y por lo tanto el convertidor vuelve a la situación de balance natural. A los $0,7 s$ se vuelve a activar el control, volviendo a producirse el balance casi perfecto de las corrientes luego de extinguido el transitorio. Esta simulación se realizó utilizando un $m_{a}=0,95$, y a pesar de que el filtro pasa bajos limita la respuesta dinámica del sistema, se obtiene una excelente respuesta final.

Como puede observarse en la Figura 3.34 la pequeña modificación en la amplitud de las portadoras por efecto del control no afecta sustancialmente el espectro de la corriente de salida del convertidor, ya que la distorsión armónica total sólo pasa de 2,24\% a 2,36\%, mientras que el desbalance total resultante es prácticamente nulo.

\subsubsection{Modulación en FPGA}

Los convertidores de potencia utilizados en la industria utilizan para su modulación y control diversos tipos de dispositivos, los más utilizados son: DSP, microcontroladores 


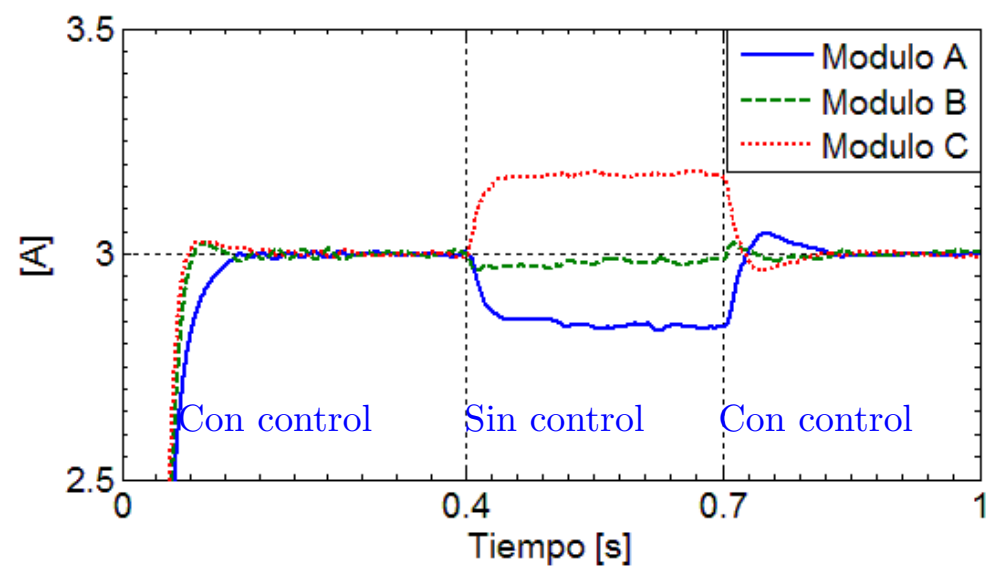

Figura 3.33: efecto del control a lazo cerrado mediante cambios en las fases relativas de portadora.

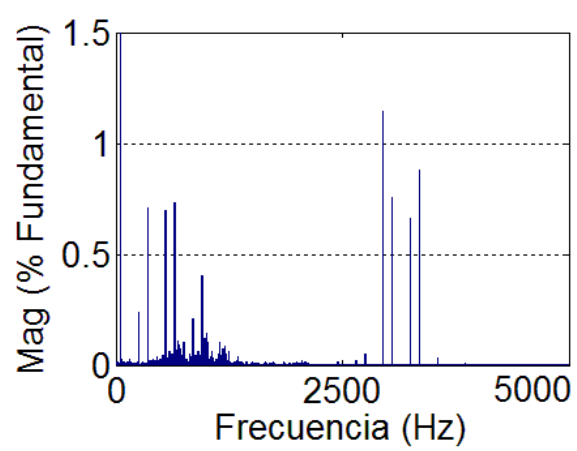

(a) Sin control: THD $=2,24 \%$, Fundamental $=7,30 \mathrm{~A}$.

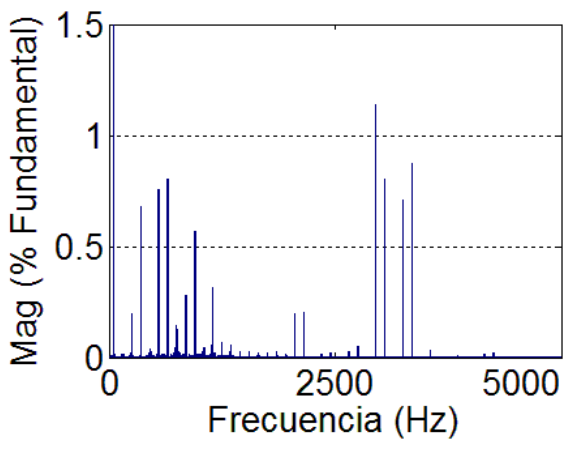

(b) con control: $T H D=2,36 \%$, Fundamental $=7,24 A$.

Figura 3.34: espectros de la corriente de salida: (a) con la modulación PSC-SPWM original, (b) con los efectos del control por amplitud $\left(m_{a}=0,95\right)$.

y FPGA. Cada tecnología tiene sus ventajas y desventajas, pero la diferencia principal entre ellas es que las FPGA no siguen pasos de programación, sino que su funcionamiento está grabado directamente en el hardware del dispositivo y esta característica las hace más inmunes a los ruidos eléctricos e interferencias electromagnéticas. Además, las FPGA son ideales para realizar tareas en paralelo, ya que no siguen un diagrama de flujo secuencial como los DSP y microcontroladores, siendo posible implementar en una misma FPGA distintas funciones independientes. La única desventaja que presentan es que requieren un knowhow muy específico y es por ello que no han sido muy utilizadas hasta el presente. Para la modulación de un convertidor MCSI Simétrico, una FPGA es la solución ideal, ya que permite realizar en forma independiente la lógica de control de cada módulo, permitiendo además bloques supervisores para las tareas adicionales como el balance de corrientes, sincronización con la red, monitoreo de variables de funcionamiento, etc. Por otro lado, el avance en las técnicas de programación de las FPGA permite la puesta en marcha de sistemas de todo grado de complejidad con relativa facilidad, aprovechando incluso las funciones de simulación 
e interfaz entre sistemas de programas como Matlab y Simulink. Es por ello que para la implementación de la lógica de modulación se utiliza un kit de desarrollo de la empresa Digilent, que contiene una FPGA XILINX Spartan 3E y varios periféricos asociados. Con una frecuencia de reloj de $50 \mathrm{MHz}$, esta FPGA es la solución óptima para obtener una gran precisión en los tiempos de conmutación y seguridad en las conmutaciones, ya que el mismo programa que va a ser bajado al dispositivo se puede simular en Matlab Simulink hasta verificar su correcto funcionamiento.

El diagrama básico de la modulación del prototipo de MCSI Simétrico de siete niveles se muestra en la Figura 3.35, donde pueden apreciarse los principales bloques descriptos en la Sección 3.3: las portadoras desplazadas en fase, la lógica SPWM y la máquina secuencial para la selección del estado cero óptimo.

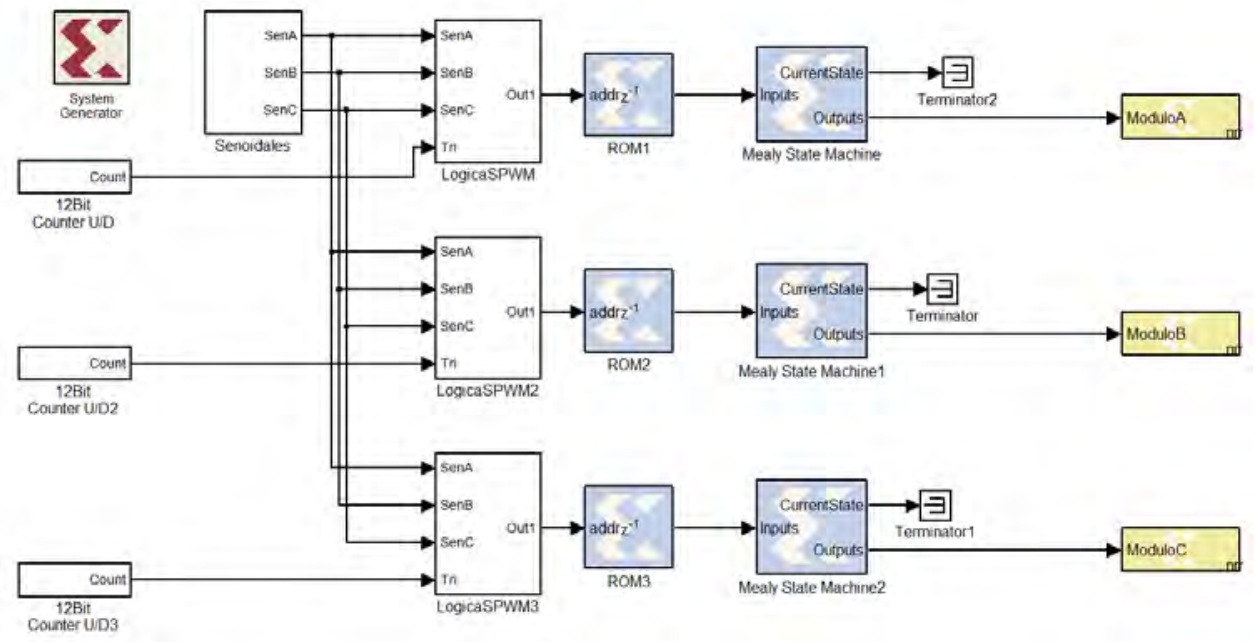

Figura 3.35: esquema de la modulación implementado en la FPGA.

El bloque Senoidales contiene un grupo de tablas con las cuales se pueden generar, con la precisión deseada, las señales de referencia que se requieren en el convertidor. Es posible generar señales sinusoidales puras para analizar la distorsión y los efectos de la conmutación a la salida, como también agregar armónicas o distorsiones y así verificar el correcto funcionamiento del prototipo como filtro activo. Para trabajar a lazo cerrado, con el convertidor inserto en un sistema eléctrico y sincronizado, el bloque de señales de referencia se reemplaza por las salidas del sistema de control, en coordenadas abc. Las portadoras triangulares de cada módulo se generan con los contadores Counter $U / D$, con la posibilidad de invertir el sentido de cuenta ( $U p /$ Down), y su inicialización se realiza desfasada $120^{\circ}$ para obetener la modulación PSC-SPWM descripta. El control de balance de las corrientes internas se realiza modificando ligeramente la salida de estos contadores, a las cuales se le suma el desfasaje requerido en el caso del control por fase (Sección 3.6.1) o se las multiplica por el factor de escala cuando se implementa el control por amplitud (Sección 3.6.2).

Los bloques LogicaSPWM contienen los bloques que realizan las ecuaciones lógicas (3.1) y su salida es un vector de seis bits con los estados activos de las llaves módulo respectivo (por ejemplo $A_{1} \ldots A_{6}$ de la Figura 3.8), de acuerdo al Cuadro 3.3. El vector resultante es codificado 
en tres bits con los mismos valores del Cuadro 3.3 a fin de simplificar la programación de la máquina secuencial y mantener el mismo valor de referencia en todo el diseño. Cabe aclarar que esta lógica agregada para la codificación será reducida al valor de mínimo costo y mínimo riesgo (Wakerly, 2006) mediante el algoritmo de simplificación del compilador Xilinx incorporado al Matlab-Simulink, y por lo tanto no implica mayor ocupación de la FPGA. El valor de estado activo se ingresa luego de su codificación a la máquina secuencial, del tipo Mealy, que está realizada en base a la Tabla de Estados y Tabla de Estados/Salidas descriptas en los Cuadros 3.8 y 3.9 .

Las Tablas de Estados se utilizan para especificar la secuencia lógica que la máquina secuencial seguirá en cada momento. Dependiendo del estado actual de la máquina, identificado en la primer columna, y del estado de las llaves que se encuentre presente en la entrada de la máquina secuencial, identificados en la primer fila, se obtendrá la secuencia siguiente que se activará en la máquina. A modo de ejemplo, la celda resaltada en el Cuadro 3.8 implica que estando la máquina en la secuencia actual $D$, cuando se detecte que la modulación ha generado un estado 3 , la siguiente secuencia de la máquina será la $C$. Puede observarse también que los estados cero no producen cambios en la secuencia activa y que se ha agregado una secuencia $I$ para la inicialización del convertidor.

En el Cuadro 3.9 se muestra la Tabla de Salidas de la máquina secuencial. Las Tablas de Salidas se utilizan para definir los valores de la salida de las máquinas secuenciales del tipo Mealy, en las cuales su salida depende de la secuencia activa en forma sincrónica y de la entrada en forma asincrónica. Esto permite variar el estado de la salida mientras se encuentra activa una secuencia, a fin de adaptar el estado cero al valor requerido. De esta manera la salida de la máquina secuencial será directamente la correspondiente a las llaves del módulo a encender. En el Cuadro 3.10 se resumen los valores decimales y binarios correspondientes a cada estado de salida de la máquina y se indican las llaves que se activarán en el módulo, de acuerdo a la definición de estados cero óptimos $\left(0_{a}, 0_{b}\right.$ y $\left.0_{c}\right)$ del Cuadro 3.4 y considerando la llave $A_{6}$ como el bit menos significativo. A modo de ejemplo, en el Cuadro 3.9 se resaltan las tres salidas válidas de la modulación mientras la máquina secuencial está en la Secuencia $C$, en la cual las entradas válidas son los estados de la modulación 1 y 3 . Cuando se detecte en la entrada un valor 1, la salida será 17, que en binario es 010001 y ello encenderá las llaves $A_{2}$ y $A_{6}$. De la misma manera, una entrada 3 produce una salida 33 , que en binario es 100001 y ello encenderá las llaves $A_{1}$ y $A_{6}$. Cuando se detecta un cero en la entrada, la salida de la máquina secuencial será un 9, cuya representación en binario es 001001, por lo que se encenderán las llaves $A_{3}$ y $A_{6}$, en correspondencia con la secuencia $\mathbf{C}$ del Cuadro 3.4. A fin de producir siempre conmutaciones seguras, evitando combinaciones de llaves inválidas que puedan producir un mal funcionamiento o falla del módulo correspondiente, se completa la tabla de salidas utilizando como valor "don't care" (Wakerly, 2006) el estado cero óptimo correspondiente a la secuencia activa, como se puede observar en los valores 9 , sin resaltar, en la fila de la secuencia $C$. Durante la inicialización del convertidor o al detectarse una falla se genera la secuencia I, para la cual la salida siempre toma el valor decimal 63, equivalente a 111111 en binario y por lo tanto todas las llaves del módulo permanecerán encendidas para garantizar el flujo de corriente de los inductores divisores. 
Cuadro 3.8: tabla de estados para la selección del estado cero óptimo.

\begin{tabular}{ccccccccc}
\hline $\begin{array}{c}\text { Secuencia } \\
\text { actual }\end{array}$ & \multicolumn{7}{c}{ Secuencia siguiente } \\
& \multicolumn{7}{c}{ Entrada: estado de la modulación) } \\
\cline { 2 - 9 } & O & 1 & 2 & 3 & 4 & 5 & 6 & 7 \\
\cline { 2 - 9 } I & I & C & A & B & E & D & F & I \\
A & A & A & A & B & F & A & A & A \\
B & B & C & B & B & B & B & A & B \\
C & C & C & B & C & C & D & C & C \\
D & D & D & D & C & E & D & D & D \\
E & E & D & E & E & E & E & F & E \\
F & F & F & A & F & F & E & F & F \\
\hline
\end{tabular}

Cuadro 3.9: tabla de salidas de la máquina secuencial tipo Mealy.

\begin{tabular}{|c|c|c|c|c|c|c|c|c|}
\hline \multirow{3}{*}{$\begin{array}{c}\text { Secuencia } \\
\text { actual }\end{array}$} & \multicolumn{8}{|c|}{ Salida: estado de las llaves del módulo } \\
\hline & \multicolumn{8}{|c|}{ Entrada: estado de la modulación } \\
\hline & 0 & 1 & 2 & 3 & 4 & 5 & 6 & 7 \\
\hline I & 63 & 63 & 63 & 63 & 63 & 63 & 63 & 63 \\
\hline $\mathrm{A}$ & 18 & 18 & 34 & 18 & 18 & 18 & 10 & 18 \\
\hline $\mathrm{B}$ & 36 & 36 & 34 & 33 & 36 & 36 & 36 & 36 \\
\hline $\mathrm{C}$ & 9 & 17 & 9 & 33 & 9 & 9 & 9 & 9 \\
\hline $\mathrm{D}$ & 18 & 17 & 18 & 18 & 18 & 20 & 18 & 18 \\
\hline $\mathrm{E}$ & 36 & 36 & 36 & 36 & 12 & 20 & 36 & 36 \\
\hline $\mathrm{F}$ & 9 & 9 & 9 & 9 & 12 & 9 & 10 & 9 \\
\hline
\end{tabular}

\subsubsection{Formas de onda experimentales}

Se demuestra en esta sección el funcionamiento del prototipo de convertidor construido, mediante la descripción detallada de las principales formas de onda. En la Figura 3.36 se muestra un ciclo completo de encendido, régimen permanente y apagado del convertidor. La corriente de salida sobre la carga es de forma sinusoidal, con mínima distorsión gracias a los siete niveles del convertidor. Puede observarse que no se presentan distorsiones durante los transitorios de encendido y apagado del convertidor. La corriente principal, en trazo amarillo, aumenta progresivamente a medida que se cargan los inductores de todo el convertidor, para ello se utiliza la función de arranque suave (soft-start) del integrado PWM de control. Esta función permite evitar sobretensiones y distorsiones a la salida durante el encendido. El 
Cuadro 3.10: asignación de salidas en función del número de estado.

\begin{tabular}{ccccc}
\hline \multirow{2}{*}{ Estado SPWM } & \multicolumn{3}{c}{ Salida Máquina Secuencial } & \multirow{2}{*}{ Llaves Activas } \\
\cline { 2 - 4 } & Estado & Decimal & Binario & \\
\hline 1 & 1 & 17 & 010001 & $A_{2} A_{6}$ \\
2 & 2 & 34 & 100010 & $A_{1} A_{5}$ \\
3 & 3 & 33 & 100001 & $A_{1} A_{6}$ \\
4 & 4 & 12 & 001100 & $A_{3} A_{4}$ \\
5 & 5 & 20 & 010100 & $A_{2} A_{4}$ \\
6 & 6 & 10 & 001010 & $A_{3} A_{5}$ \\
\hline & $0_{a}$ & 36 & 100100 & $A_{1} A_{4}$ \\
0 & $0_{b}$ & 18 & 010010 & $A_{2} A_{5}$ \\
& $0_{c}$ & 9 & 001001 & $A_{3} A_{6}$ \\
\hline
\end{tabular}

valor de referencia (setpoint) de la corriente principal es aumentado al final de la simulación, verificando que el convertidor presenta una excelente respuesta dinámica frente cambios en la corriente de la fuente principal. El trazo en verde muestra la corriente de uno de los inductores divisores, donde puede observase que, a pesar del ripple presente, se mantiene balanceada con un valor medio de $1 / 3$ de la corriente principal. El trazo restante muestra la tensión en una llave, pudiendo verificar que gracias a que tanto el arranque como el apagado son controlados no se presentan sobretensiones.

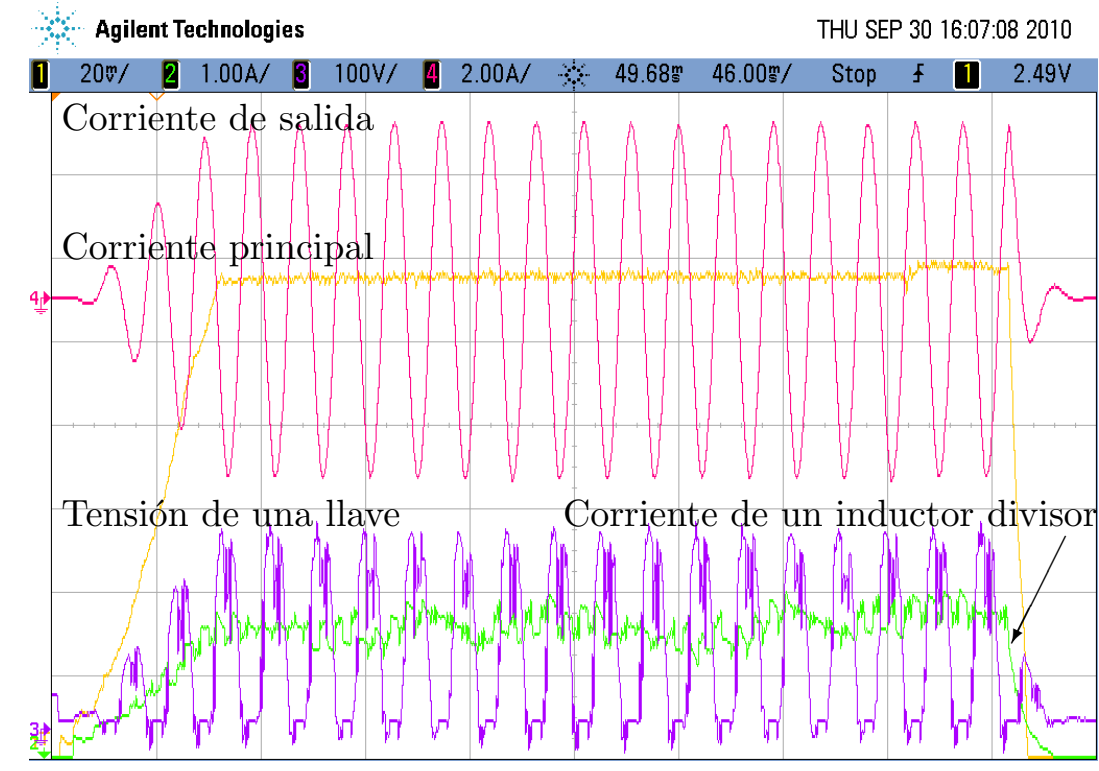

Figura 3.36: corriente de salida de una fase, corriente principal, tensión sobre una llave y una de las corrientes internas para un ciclo de encendido, regimen permanente y apagado del convertidor.

En la Figura 3.37 puede observarse en detalle la tensión de la llave $A_{4}$, donde se aprecia que no se producen sobretensiones, gracias al cuidadoso diseño del conexionado (layout) 
y a la acción de un pequeño banco de capacitores auxiliares. La tensión en la llave tiene la forma característica esperada, observándose que la llave se mantiene encendida (tensión aproximadamente cero) mientras la corriente pasa por su mayor valor negativo y durante el resto del ciclo mantiene un ripple dictado por la conmutación del resto de las llaves del convertidor y la carga, por ello su envolvente tiene forma sinusoidal aunque ligeramente desfasada con respecto a la corriente, debido al factor de potencia no unitario de la carga.

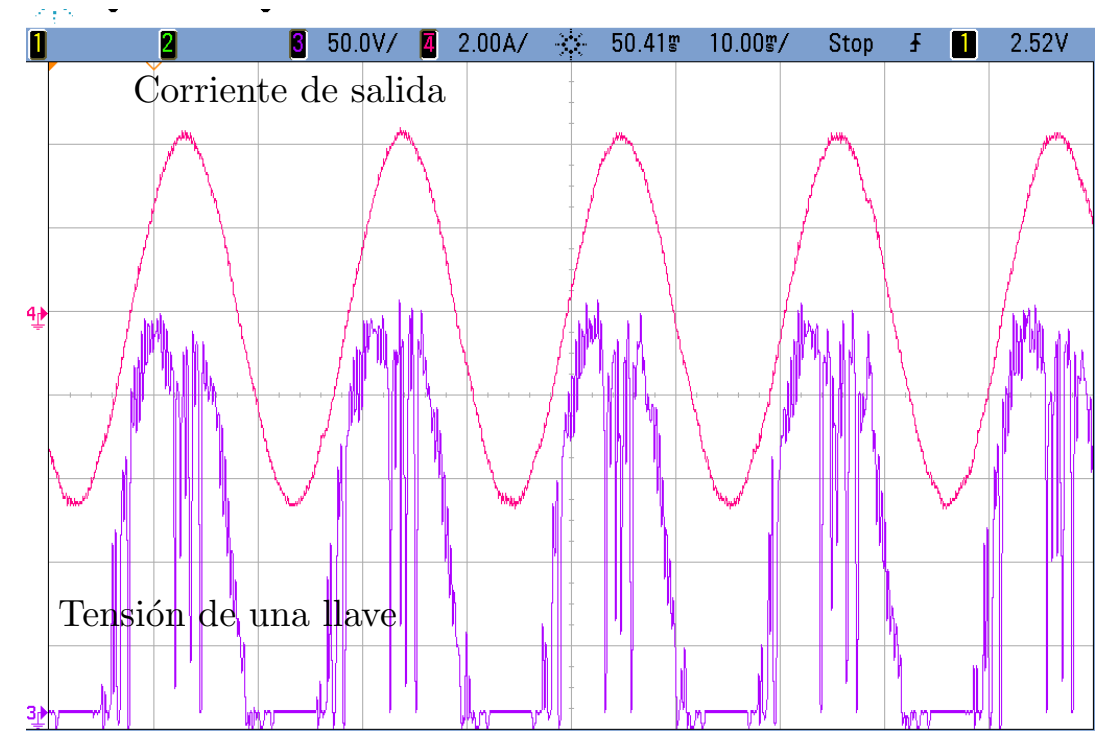

Figura 3.37: detalle de la tensión en una llave en relación con la corriente de salida, la forma de onda sobre la llave depende de la carga del convertidor.

En la Figura 3.38 puede observarse la diferencia de fase entre la corriente generada por el convertidor y la tensión en la carga debido a la componente inductiva. Si bien la tensión tiene una componente de alta frecuencia, remanente del filtrado por el banco de capacitores, el ripple se mantiene acotado dentro de los límites normales de operación de una red eléctrica definidos por IEEE (1993). Se agregaron el gráfico la corriente principal y una de las corrientes internas como referencia.

La respuesta dinámica del convertidor frente a cambios en la carga es una característica muy importante a la hora de evaluar sus aplicaciones en condiciones de carga variable, como por ejemplo un filtro activo en un sistema de potencia. Frente a un cambio en la carga, tanto la corriente principal, como las corrientes internas de cada módulo y la corriente de carga deben permanecer constantes y no se deben presentar sobretensiones. Para verificar la respuesta del prototipo de MCSI Simétrico construido se realiza un ensayo de arranque, cambio de carga y apagado, cuyos resultados se muestran en la Figura 3.39. El ensayo consta de cuatro etapas, primero se arranca el convertidor a plena carga, luego se reduce la componente resistiva de la carga al $50 \%$, luego se vuelve a conmutar a plena carga y por último se apaga el convertidor. Se puede observar que las corrientes permanecen constantes, que la tensión de salida se reduce a la mitad y que no se producen sobretensiones ni distorsiones. Esto implica una excelente respuesta dinámica frente a cambios en la carga por lo que el convertidor resulta adecuando para su uso en sistemas que puedan tener grandes variaciones del punto de trabajo, corriente 


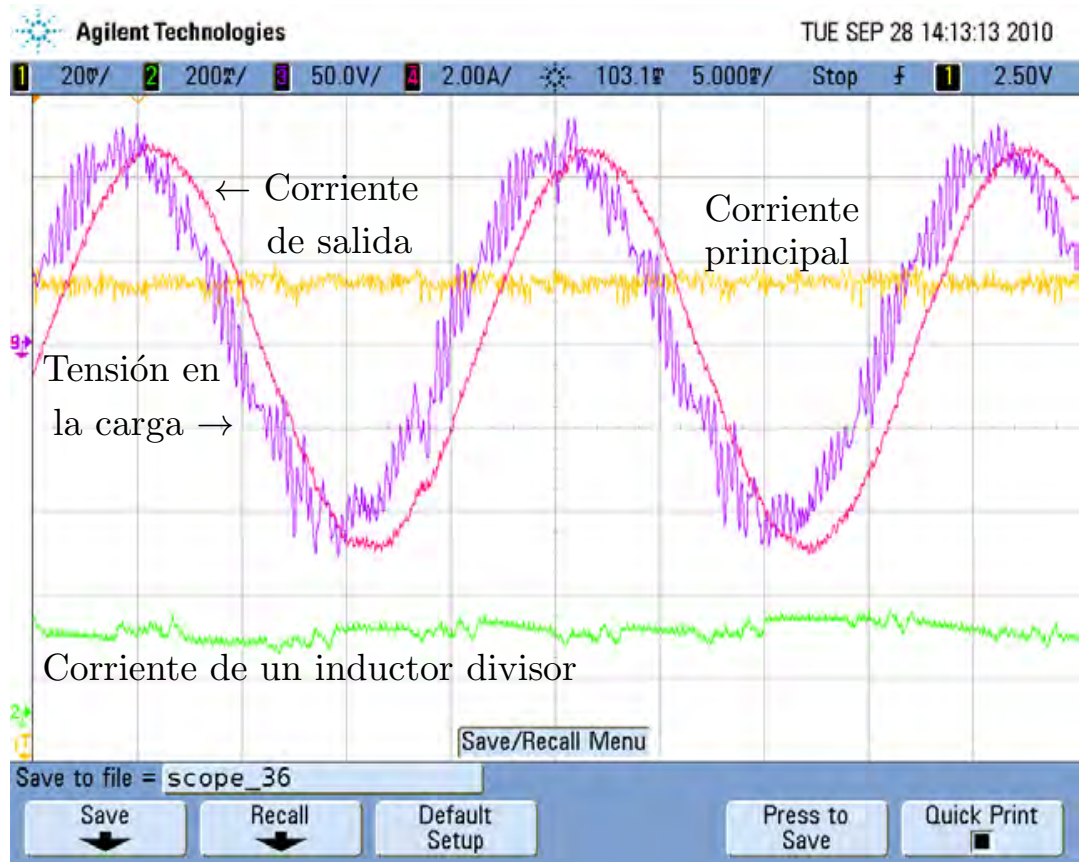

Figura 3.38: detalle del funcionamiento en régimen permanente, donde se observa el desfasaje entre la tensión y la corriente en la carga debido a su componente inductivo y que tanto la corriente principal como las internas permanecen balanceadas, con un pequeño ripple.

de carga o potencia de salida. En la Figura 3.40 puede observarse un detalle del ensayo de reducción de carga, donde se ha utilizado sólo carga resistiva a fin de obtener únicamente la respuesta del convertidor, evitando cualquier interferencia que la conmutación de una carga inductiva pueda producir.

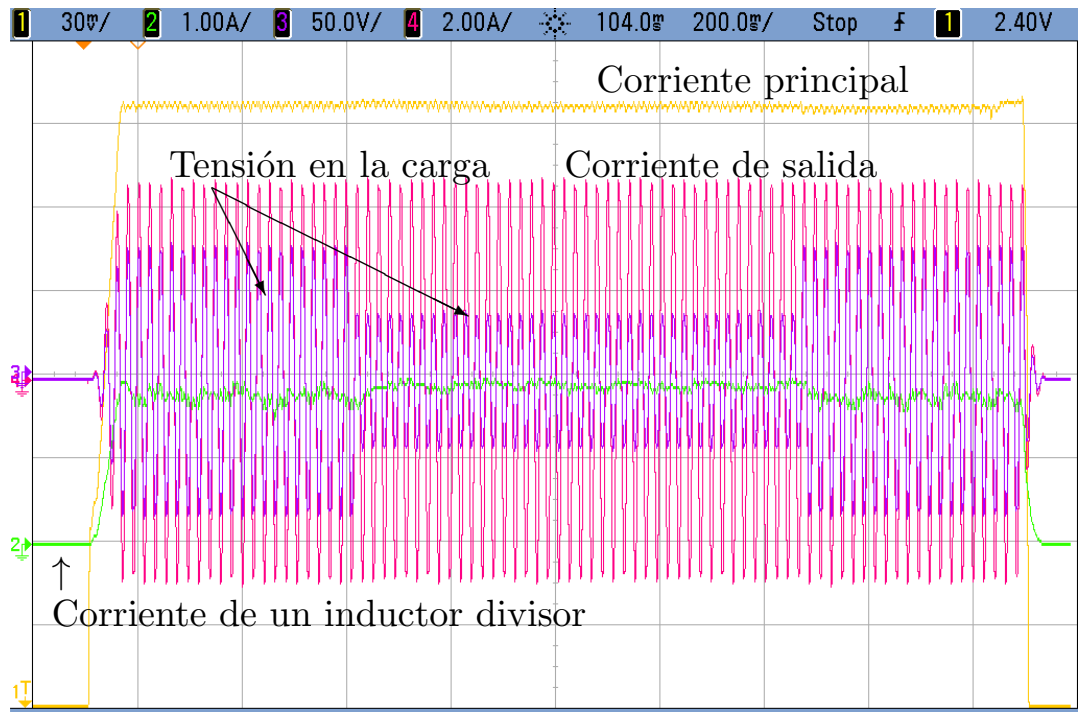

Figura 3.39: salto en la resistencia de carga en un ciclo de encendido, régimen permanente y apagado del convertidor. Corriente principal, corriente de salida de una fase, tensión de una fase de salida y una de las corrientes internas. 


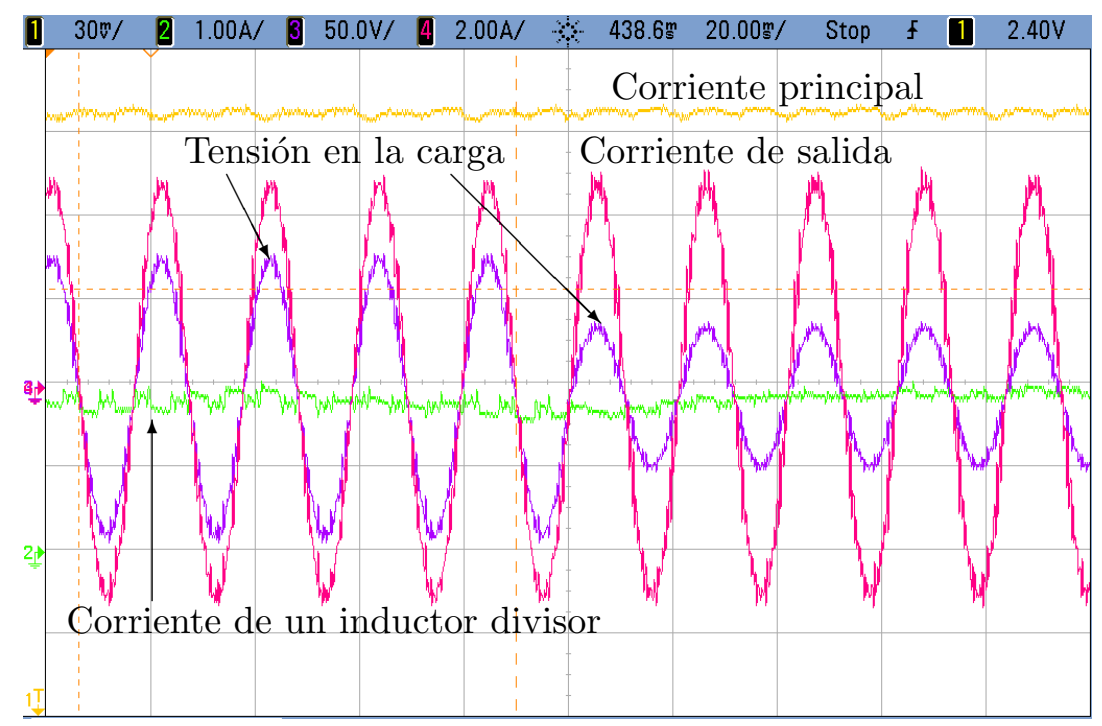

Figura 3.40: detalle del ensayo de salto de carga. Corriente principal, corriente de salida de una fase, tensión de una fase de salida y una de las corrientes internas.

La posibilidad de generación de armónicas es otra medida de la respuesta del convertidor, ya que es una característica indispensable en un filtro activo de potencia. En la Figura 3.41 se muestra un ensayo del convertidor donde se suma a la fundamental componentes de tercera y quinta armónicas. Se puede observar en la Figura 3.41(a) que las tres corrientes de salida tienen las mismas deformaciones y que no se producen otro tipo de distorsiones, mientras que en la Figura 3.41(b) se tiene el espectro en frecuencias con el contenido de fundamental, $3^{r a}$ y $5^{\text {ta }}$ armónicas.

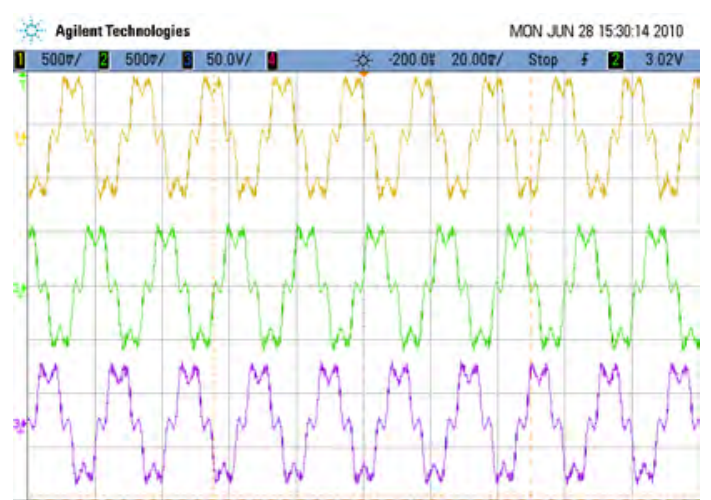

(a)

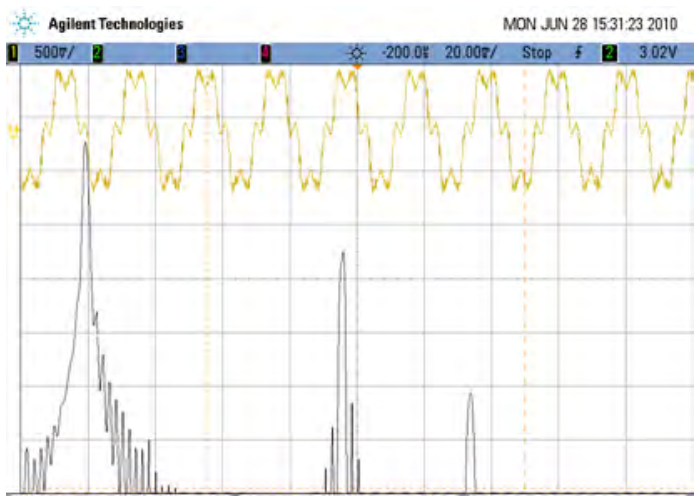

(b)

Figura 3.41: respuesta dinámica del convertidor real, inyección de 3era y 5ta armónicas. 


\subsection{Conclusiones}

En este capítulo se diseñó un convertidor fuente de corriente de siete niveles con una topología MCSI Simétrico de tres módulos. Se analizó su funcionamiento, la estructura circuital, las características de conmutación de las llaves, se adaptó una técnica de modulación y se diseñó el control del convertidor en conjunto.

Se verificó la correcta aplicación de la modulación PSC-SPWM a la topología seleccionada, utilizando además la técnica Tri-Logic para adaptar las señales de disparo a los requerimientos de un MCSI. Esto resulta en una lógica de modulación de muy rápida respuesta, compacta, con posibilidad de procesamiento en paralelo y fácil de implementar en una FPGA, lo que minimiza los retardos en los tiempos de cálculo, acotando los errores de muestreo y reduciendo la distorsión en la corriente de salida.

Se verificó que el desplazamiento de las portadoras de la modulación PSC-SPWM produce un balance natural de las corrientes internas del convertidor, en forma análoga al efecto producido en algunas estructuras MVSI. Si bien este balance es débil, puede ser afectado por perturbaciones y tiene un pequeño error en estado estacionario, su efecto es lo suficientemente fuerte como para permitir un análisis básico del convertidor sin requerir complejas estructuras de control.

El agregado de una máquina secuencial lógica especialmente diseñada para la topología permite seleccionar el estado cero óptimo en cada instante de conmutación, logrando así reducir drásticamente la cantidad de conmutaciones por ciclo de las llaves y, por ende, la disipación de potencia del convertidor resultante. Además permite aumentar la vida útil de las llaves y sus circuitos auxiliares, reduce los requerimientos de potencia de los circuitos de disparo y minimiza la emisión de interferencia electromagnética (EMI).

Del análisis de la forma de conmutación, los caminos de conducción de corriente y las tensiones en cada grupo de tres llaves que conmutan la corriente de un inductor divisor se obtiene que el efecto de los componentes parásitos es muy distinto al típico de los convertidores de tensión. Las inductancias parásitas en serie con los inductores divisores o la carga no producen ningún efecto sobre el convertidor, simplificando el diseño de los conexionados. Se deben minimizar las capacidades parásitas entre llaves, para evitar que la conmutación de una afecte a las otras, ya que sus bornes no están sujetos a una tensión fija del bus de continua como en los MVSI. Pero el efecto más importante ocurre al minimizar las inductancias parásitas en el camino de conmutación de la corriente de un inductor divisor, ya que esto produce un efecto de conmutación suave favoreciendo el paso de corriente de una llave a la siguiente (en la secuencia de conmutación) y minimizando así la potencia de pérdidas por conmutación en las llaves. Esta característica, sumada a la reducción del número de conmutaciones por ciclo, permiten escalar los resultados obtenidos a convertidores de muy alta potencia. Se construyó un prototipo de $3 \mathrm{KW}$, directamente escalable a mayores potencias, en el cual se validaron las suposiciones teóricas y las simulaciones, verificando que las llaves prácticamente no disipan potencia.

Se ha demostrado mediante simulaciones el correcto funcionamiento de la estructura de 
tolerancia a fallas presentada, basada en el agregado de un módulo de respaldo en caliente y las llaves de protección. De esta manera es posible aumentar la confiabilidad del convertidor, tolerando una falla en cualquiera de sus componentes sin producir efectos severos a la salida. El agregado de un módulo de respaldo y la posibilidad de realizar su puesta en marcha en cualquier momento, manteniendo en servicio al convertidor, permite la realización de tareas de mantenimiento preventivo y el ciclado de componentes para evitar su fatiga, características éstas indispensables en convertidores de muy alta potencia a utilizar en sistema que requieren máxima disponibilidad y MTBF, como ser los sistemas eléctricos.

Se diseñaron dos métodos para el control del balance de las corrientes internas del convertidor, ambos basados en introducir pequeñas modificaciones en la modulación PSCSPWM. Se demostró que es posible realizar el control de las corrientes sin afectar significativamente la distorsión de la corriente de salida, por lo cual es posible implementar el control propuesto en aplicaciones de sistemas eléctricos y otras donde se requiera generar mínima distorsión. Si bien ambas técnicas permiten realizar el balance con resultados similares, el control por variación de amplitud de las portadoras presenta una ganancia variable con el punto de trabajo del convertidor, presentando una mayor sensibilidad cuando la señal a generar está en niveles de $m_{a}$ mayores a aproximadamente 0,6. Dado que el control se realiza con controladores PI simples, es posible aprovechar esta diferencia para realizar un controlador que pase de un método a otro dependiendo del punto de trabajo del convertidor y utilizar así cada método en punto de máximo rango dinámico.

De las pruebas de cambio de punto de trabajo y saltos de carga se deduce que la topología MCSI Simétrico posee una excelente respuesta dinámica, pudiendo tolerar cambios bruscos en su punto de trabajo y grandes saltos de carga sin producir distorsiones en las corrientes de salida.

La suma de las características presentadas resultan en un convertidor muy prometedor para su utilización en aplicaciones de filtros activos e interfaz con sistemas de energías alternativas como los que se presentan en el capítulo siguiente. 


\section{Capítulo 4}

\section{Aplicaciones}

En este capítulo se investiga la factibilidad de utilizar la topología MCSI seleccionada en aplicaciones de redes eléctricas, especialmente aquellas en las que el uso de energía renovables presenta nuevos desafíos debido a su inestabilidad y predictibilidad de corto plazo. En sistemas eléctricos donde una considerable porción de la generación proviene de fuentes renovables, como solar o eólica, se pueden presentar variaciones en la potencia disponible, excesos, faltantes o sub-armónicos que afectan seriamente la calidad del servicio eléctrico. Dependiendo de la frecuencia y duración esperadas de las perturbaciones se pueden utilizar diferentes métodos para suavizar los picos de generación y compensar los faltantes, almacenando el exceso de energía para luego restituirlo a la red cuando sea necesario. Para ello es necesario contar con un sistema de almacenamiento, que puede ser realizado mediante capacitores, baterías y dispositivos superconductores (SMES) entre otros. Pero en aquellas aplicaciones donde se requiere la mayor seguridad ambiental, gran confiabilidad y extensa vida útil el uso de la tecnología de hidrógeno es una de las mejores opciones. Por otra parte, las armónicas introducidas por las cargas afectan seriamente la calidad de los sistemas de distribución. Por otro lado, si se requiere maximizar la eficiencia es indispensable corregir el factor de potencia del sistema. Ambos problemas pueden atacarse a nivel de media tensión agregando al sistema de distribución un filtro activo en derivación basado en un MCSI.

En particular se analizaron dos aplicaciones específicas:

- Almacenamiento de energía en forma de $H_{2}$ : interfaz para generación y reutilización (Aguirre et al., 2011a; Aguirre y Valla, 2011; Aguirre et al., 2011b, 2012b).

- Acondicionamiento de redes eléctricas: corrección de factor de potencia, filtro activo (Aguirre et al., 2010a,c).

Los resultados del análisis presentado pueden extrapolarse fácilmente a otros casos gracias a la versatilidad y respuesta dinámica del convertidor bajo estudio (Aguirre et al., 2013b,d). 


\subsection{Interfaz para almacenamiento de energia en forma de $H_{2}$}

El almacenamiento de energía en forma de hidrógeno, así como su transformación desde y hacia energía eléctrica, es una excelente opción en aplicaciones donde el cuidado del medio ambiente es una restricción importante de diseño. Esto se debe a su relativa buena eficiencia, confiabilidad, bajo mantenimiento y la casi ausencia de contaminación que su uso genera. Para la obtención de hidrógeno se pueden utilizar electrolizadores, los que convierten energía eléctrica y agua en hidrógeno y oxígeno gaseosos. Un electrolizador de alta presión genera hidrógeno directamente a altas presiones (> 300bar) con gran eficiencia, ya que no requieren etapas posteriores de compresión (Lauretta, 2005). A su vez, el hidrógeno puede ser utilizado para generar energía eléctrica mediante el uso de una celda de combustible. Existen al momento diferentes tipos de celdas de combustible, por ejemplo: alcalinas (AFC), óxido sólido (SOFC) y membranas de intercambio de protones (PEMFC); de las cuales se dispone de una gran variedad de estudios y modelos matemáticos en la literatura, lo que permite simular adecuadamente su funcionamiento. Tanto los electrolizadores, como las celdas de combustible funcionan mejor si son manejadas desde una fuente de corriente, por ello en los esquemas tradicionales se utilizan etapas Buck para alimentar a los electrolizadores y etapas Boost para cargar a las celdas de combustible.

Cuando hay disponibilidad de energía eléctrica, por baja de demanda o exceso de generación por métodos renovables, un electrolizador permite absorber potencia activa del sistema eléctrico y generar hidrógeno para que sea almacenado, con mínimos riesgos ambientales. Cuando la generación de energía eléctrica cae por debajo de los requerimientos de la carga, ya sea por una reducción momentánea del viento (generación eólica), porque se nubla o llega el atardecer (generación solar) o por un aumento momentáneo de la carga, el hidrógeno puede ser utilizado en una celda de combustible para mantener la potencia eléctrica disponible en el sistema eléctrico. Configuraciones de almacenamiento como las descriptas pueden ser utilizadas para mejorar la calidad de energía en sistemas débiles y/o altamente dependientes de la generación distribuida y para proveer energía ininterrumpida a una carga. Por otro lado, el hidrógeno puede almacenarse por largos períodos de tiempo por lo que es posible proporcionar energía segura independientemente del tiempo de latencia, lo que no ocurre con baterías, las que deben ser sometidas a mantenimiento periódico a pesar de no haber sido utilizadas. A fin de controlar adecuadamente tanto la generación de hidrógeno como de energía eléctrica, y además alcanzar la máxima eficiencia posible, es indispensable el uso de convertidores electrónicos con capacidad de intercambiar energía en forma bidireccional.

\subsubsection{Electrolizadores}

Un electrolizador es un dispositivo electroquímico que utiliza energía eléctrica para disociar el hidrógeno del agua. Los electrolizadores de alta presión (Lauretta, 2005) presentan la ventaja especial de producir hidrógeno que puede ser almacenado directamente a alta presión sin la necesidad de etapas compresoras intermedias. Esto aumenta notablemente la eficiencia del sistema, además de evitar partes móviles, incrementando la confiabilidad y 


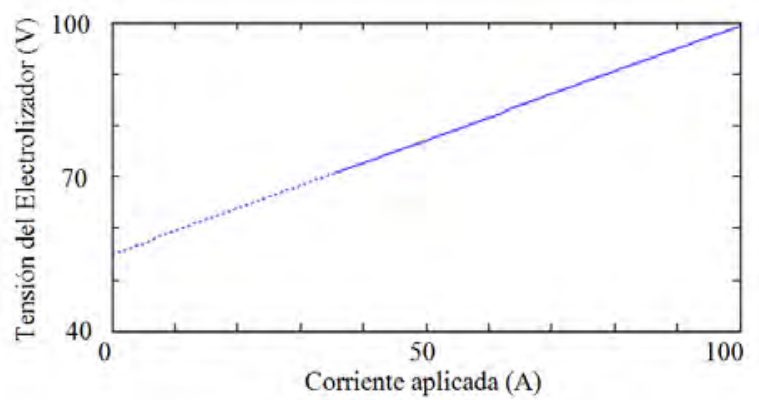

Figura 4.1: curva característica tensión vs. corriente del electrolizador de alta presión.

reduciendo las necesidades de mantenimiento. El modelo matemático del electrolizador se puede obtener del trabajo de Smoglie y Lauretta (2010), verificado mediante resultados experimentales, en el que las componentes parásitas de la corriente consumida por el electrolizador se pueden despreciar cuando el punto de operación está en la zona de alta corriente. De esta manera, la relación entre la corriente consumida y la tensión presente en los bornes puede modelarse mediante la expresión

$$
V=i \rho n l\left(\frac{1}{\sigma}+\frac{A}{\rho l}\right)+n B
$$

donde $\sigma, \rho, l$ y $n$ son parámetros físicos que dependen de la construcción y materiales del electrolizador. Las constantes $A$ y $B$ fueron obtenidas por Smoglie y Lauretta (2010) mediante resultados experimentales en un prototipo, siendo $A=0,0061$ y $B=1,8311$, por lo que el modelo resulta:

$$
V=0,4439 i+54,93
$$

En la Figura 4.1 se muestra la curva característica tensión-corriente linealizada del electrolizador de alta presión utilizado. Se destaca en línea llena la región de operación donde pueden despreciarse las corrientes parásitas.

\subsubsection{Celdas de combustible.}

Una celda de combustible, cuyo diagrama esquemático se presenta en la Figura 4.2, es un dispositivo electroquímico que convierte energía química en energía eléctrica mediante una reacción química en un electrolito, generando agua y calor como subproductos (Ellis et al., 2001). Se considerará en este trabajo una celda de combustible PEMFC dado que presenta características de operación adecuadas para los niveles de tensión, corriente y potencia considerados. Una celda de combustible típica puede estar construida con un conjunto de muchos elementos (900), con una potencia nominal de decenas de kilowatt (50kW) a una tensión adecuada para su transformación a trifásica de red mediante un solo convertidor $\left(625 \mathrm{~V}_{C C}\right)$. Conjuntos como los descriptos pueden presentar una eficiencia relativamente baja (menor al $60 \%$ ) lo que es todavía una desventaja de la tecnología, aunque se están 
realizando extensas investigaciones al respecto y se espera que en un futuro cercano la eficiencia pueda aumentarse considerablemente. Estas celdas están emergiendo como una de las más prometedoras tecnologías a la hora de reemplazar los combustibles fósiles para la generación de energía eléctrica, gracias a la ausencia de contaminantes y a la facilidad y seguridad de operación.

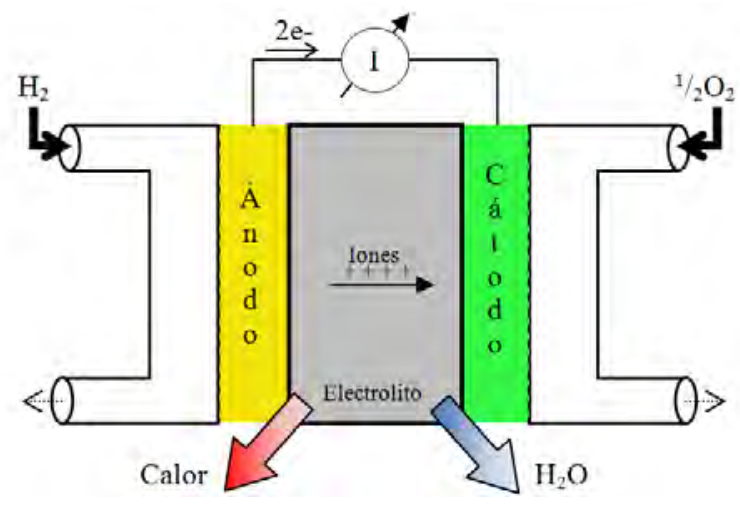

Figura 4.2: diagrama esquemático de una celda de combustible.

En (Friede et al., 2004; Xin y Khambadkone, 2003; Sirisukprasert y Saengsuwan, 2008; Ellis et al., 2001; Kunusch et al., 2010) se pueden encontrar modelos matemáticos detallados, donde se observan tres áreas de operación primarias: Zona de Activación, Zona Óhmica y Zona de Decaimiento, siendo preferible la operación en la región Óhmica debido a la mayor eficiencia (Sirisukprasert y Saengsuwan, 2008). Se puede encontrar un modelo matemático, apto para su simulación por computadora, en la herramienta "SimPowerSystems" de Matlab/Simulink (Souleman et al., 2009). En la Figura 4.3 se presenta la curva característica de corriente vs. tensión de la PEMFC analizada.

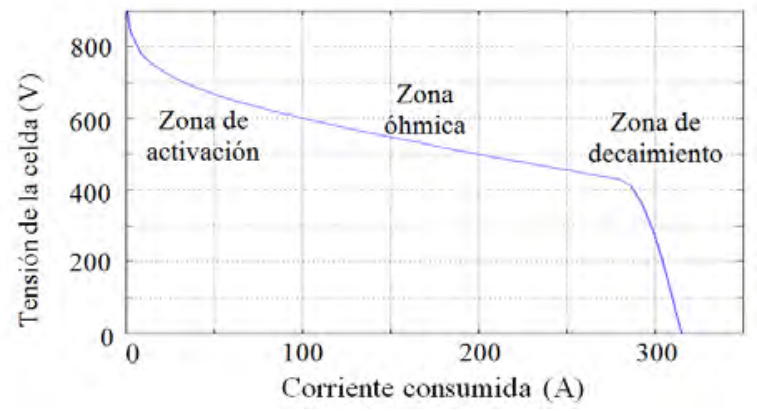

Figura 4.3: característica tensión vs. corriente de una celda de combustible PEMFC típica.

Una desventaja de las celdas de combustible es que la tensión que proporcionan no es constante, sino que varía según la corriente consumida por la carga. Por lo tanto, es indispensable la utilización de un convertidor electrónico de potencia para realizar la interfaz entre la PEMFC y el sistema eléctrico, logrando independizarse así del punto de operación. Además, el convertidor permite ajustar la corriente tomada de la celda para llegar lo más cerca posible al punto de mayor eficiencia (Souleman et al., 2009; Sirisukprasert y Saengsuwan, 2008). 


\subsubsection{Interfaz con el sistema de almacenamiento de $H_{2}$}

En los esquemas tradicionales se utilizan rectificadores, controlados o no, para alimentar con energía eléctrica al electrolizador. Estos rectificadores introducen armónicos en la red y no pueden ser controlados adecuadamente para adaptarse a las necesidades dinámicas de las redes inteligentes (Smart Grids). A su vez, la salida de la celda de combustible se hace pasar por una etapa elevadora (Boost) por dos razones: para que la celda esté cargada con una fuente de corriente y para elevar la tensión. La etapa elevadora carga un capacitor que conforma el bus de continua de un convertidor VSI, el que inyecta la energía a la red a través de inductores de acoplamiento (Alepuz et al., 2006; Wang et al., 2006). Se propone un esquema compuesto por sólo un convertidor MCSI, el que presenta una corriente prácticamente constante en su bus de continua, pudiendo alimentar a un electrolizador de alta presión y recibir corriente de una celda de combustible, intercambiando energía con la red eléctrica sin necesidad de componentes adicionales, con la excepción de una llave electrónica de bajas pérdidas para la interconexión de la celda o el electrolizador, que puede construirse con transistores MOS o IGBT de potencia, con el agregado de diodos en serie para garantizar el bloqueo de tensión bidireccional. Dado que la frecuencia de operación de esta llave es prácticamente cero, las pérdidas en la misma pueden hacerse completamente despreciables.

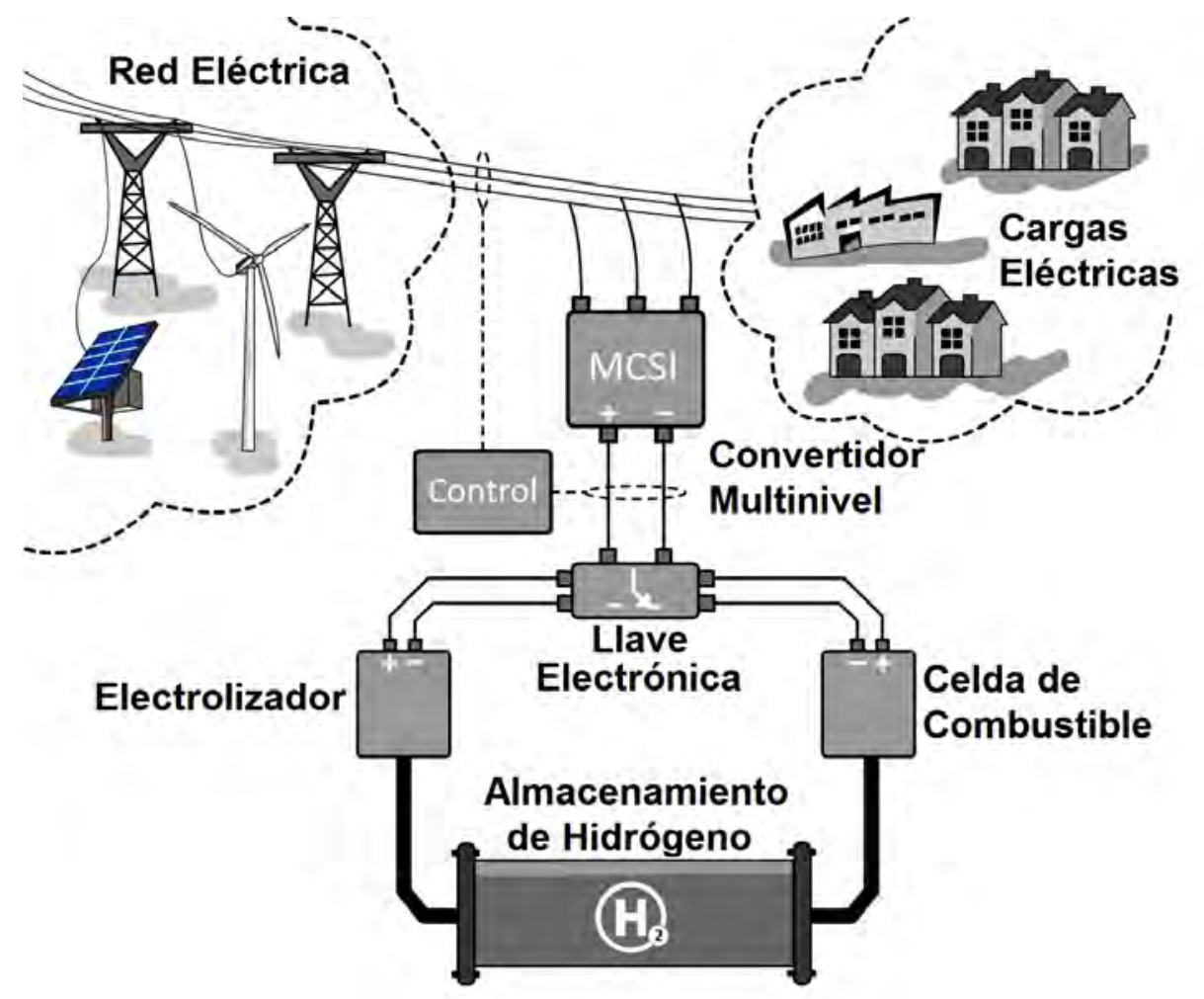

Figura 4.4: diagrama de bloques del sistema propuesto.

El convertidor MCSI se conecta en paralelo con la red eléctrica de distribución, como se muestra Figura 4.4, acoplado mediante un pequeño banco de capacitores para evitar sobretensiones durante las conmutaciones de corriente. Una llave electrónica de bajas pérdidas 
conecta el electrolizador o la celda al bus de continua, según corresponda a la dirección deseada del flujo de potencia. El bloque de control, mostrado en la Figura 4.5, consiste en dos controladores PI con distintos parámetros de sintonía de acuerdo al dispositivo controlado, permitiendo adaptar la respuesta de todo el sistema a las distintas ganancias y dinámicas del electrolizador y la celda de combustible. Cada bloque PI controla la potencia activa intercambiada entre el convertidor y el sistema, modificando de esta manera la corriente del bus de continua y la energía almacenada en el MCSI. Los controladores se mantienen en modo seguimiento (tracking) mientras están desactivados, para evitar saltos o discontinuidades al pasar de un modo al otro. El bloque de control está sincronizado con la red mediante un Lazo enganchado en fase (PLL) trifásico.

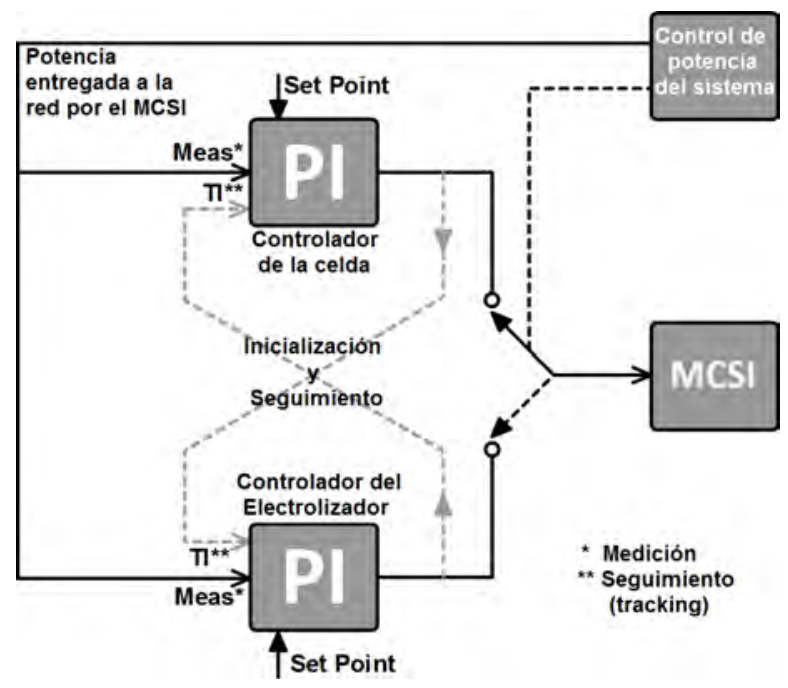

Figura 4.5: detalle del bloque de control.

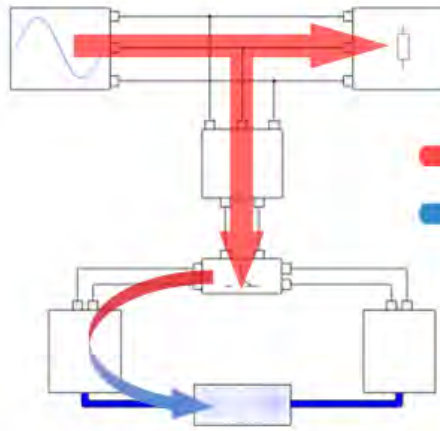

a)

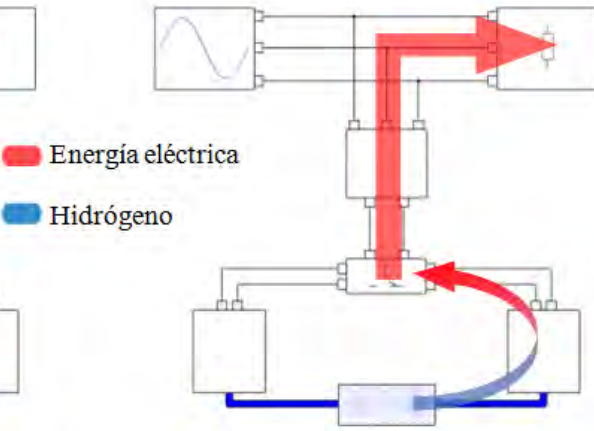

b)

Figura 4.6: flujo de energía en el sistema, a) produciendo hidrógeno, b) generando energía eléctrica.

En la Figura 4.6 puede observarse un detalle del intercambio de energía en el sistema para los dos modos de operación. Cuando se presenta generación en exceso, se conecta el electrolizador al bus de continua del convertidor. La potencia activa puede circular a través del convertidor y la llave electrónica, y transformarse en hidrógeno en el electrolizador. En 
estas condiciones, la potencia requerida por la carga será provista por la red eléctrica. Cuando el nivel de generación no alcanza para cubrir los requerimientos de la carga, se desconecta el electrolizador y se conecta el bus de continua a la celda de combustible. La celda convierte el hidrógeno en energía eléctrica, entregándose ésta al sistema a través de la llave electrónica y el convertidor.

Mediante modelos detallados de Matlab/Simulink se realizaron simulaciones del sistema propuesto completo, en diversas situaciones típicas, a fin de analizar la respuesta del convertidor y su interacción con la red eléctrica. En la Figura 4.7 se muestra la respuesta del sistema a tres situaciones típicas, donde:

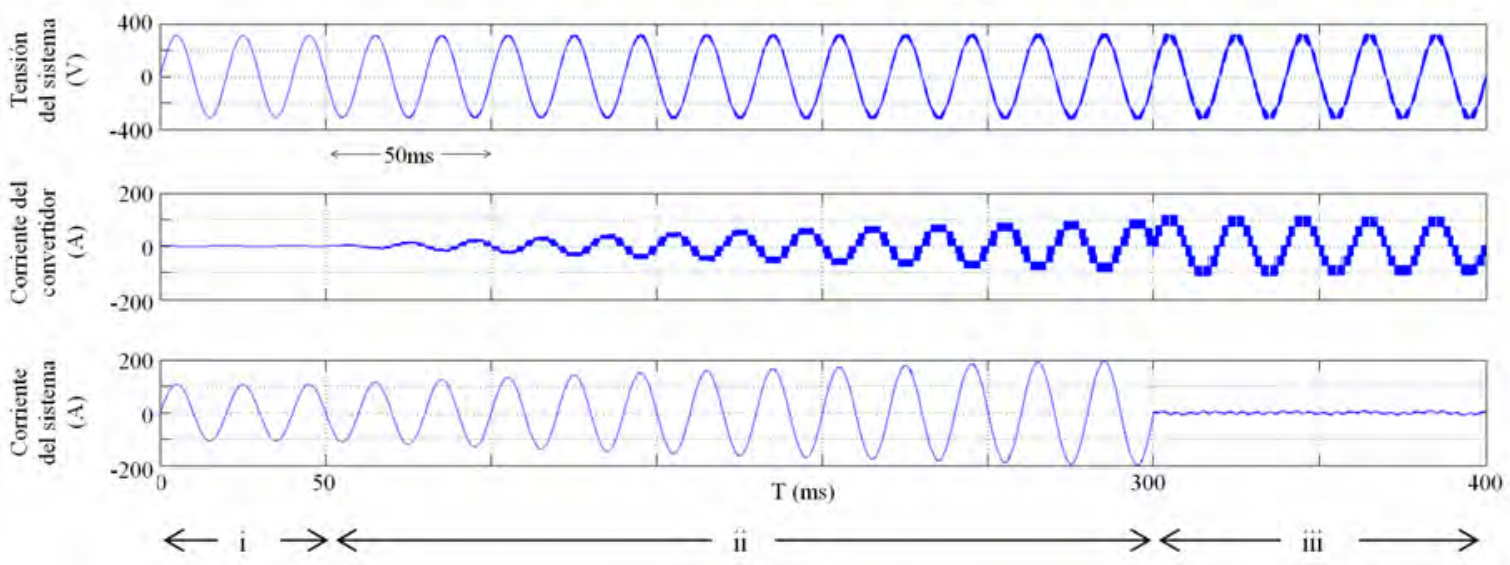

Figura 4.7: simulación del comportamiento del sistema ante situaciones típicas.

I al principio de la simulación, la red eléctrica provee la energía para la carga. Tanto el convertidor, como el sistema de hidrógeno asociado están desconectados.

II a los $50 \mathrm{~ms}$, el convertidor se conecta a la red y la llave electrónica conecta al electrolizador del lado de continua. El convertidor toma potencia activa de la red, alimentando al electrolizador. Puede observarse como tanto la corriente a través del convertidor como la provista por el sistema aumentan a medida que los inductores del MCSI se cargan.

III a los $300 \mathrm{~ms}$, la llave electrónica desconecta al electrolizador y la celda de combustible queda conectada al lado de continua del convertidor. El control invierte el sentido del flujo de potencia a través del convertidor, cambiando en forma casi instantánea la fase de las señales de referencia. La corriente provista por el sistema se anula, siendo la energía requerida por la carga provista por la celda de combustible, consumiendo el hidrógeno almacenado.

Si bien el convertidor inyecta a la red componentes de alta frecuencia, que pueden notarse en la forma de onda de la tensión resultante del sistema, estas perturbaciones no interfieren con el normal funcionamiento del sistema eléctrico, manteniendo en todo momento una distorsión armónica total (THD) menor al 5\%, límite impuesto según el estándar "Std. 519 1992” por IEEE (1993), aún considerando hasta la armónica número 50. 
En la Figura 4.8 se muestra un detalle de las formas de onda resultantes de la conexión de un electrolizador y el MCSI a la red. La corriente consumida por el MCSI aumenta a medida que sus inductores se cargan, hasta llegar al valor nominal donde se estabiliza. Durante el transitorio de arranque el MCSI está en sobremodulación para acelerar el proceso de carga, pero al llegar a la corriente nominal se comienzan a generar normalmente los siete niveles de salida mediante la modulación PSC-SPWM. La corriente del sistema parte de un nivel inicial, correspondiente al consumido por la carga, y aumenta gradualmente para proveer la energía consumida por el electrolizador a través del MCSI. Dado que el electrolizador consume sólo potencia activa, la corriente que el convertidor inyecta a la red resulta en contrafase con la tensión del sistema en concordancia con el consumo de potencia activa, mientras que la corriente del sistema se mantiene en fase con la tensión. Puede observarse que aparece una pequeña distorsión en la tensión del sistema, debida a las componentes de alta frecuencia inyectadas por el convertidor, cuyo valor está por debajo de los límites requeridos por IEEE (1993).

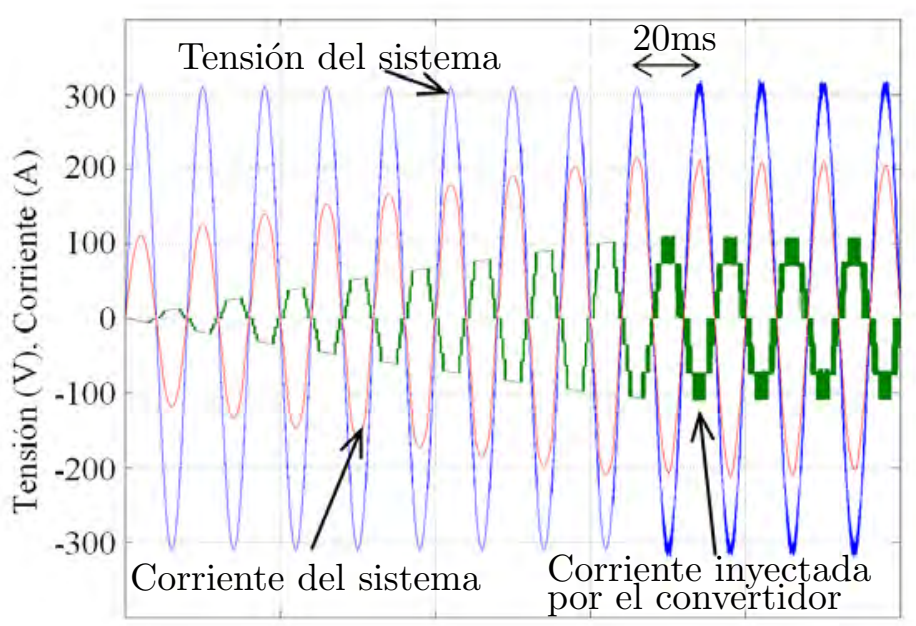

Figura 4.8: formas de onda durante la conexión del electrolizador y el MCSI a la red.

En la Figura 4.9 se muestra la potencia entregada por el sistema eléctrico al MCSI, que éste entrega a su vez al electrolizador y que aumenta en la misma medida que la corriente del convertidor. Una vez estabilizado el punto de trabajo del conjunto convertidorelectrolizador, la potencia activa consumida se mantiene constante en el valor requerido según la corriente continua que se esté suministrando al electrolizador. La potencia activa en la carga se mantiene constante en su valor inicial ya que no se afecta la tensión del sistema eléctrico.

En la simulación de la Figura 4.10 se analiza el conexionado del electrolizador y el MCSI a un sistema eléctrico con intercambio de potencia activa y reactiva. A los $0,1 s$ se conecta el MCSI al sistema y hasta los primeros $250 \mathrm{~ms}$ de simulación se mantiene la referencia de potencia activa en $55 K W$ y la de potencia reactiva en cero (Aguirre et al., 2010b). A los $250 \mathrm{~ms}$ se lleva la referencia de potencia reactiva a $15 \mathrm{KVAr}$. Puede observarse como la potencia reactiva entregada por el sistema se reduce en la misma medida que la potencia entregada por el MCSI aumenta. 

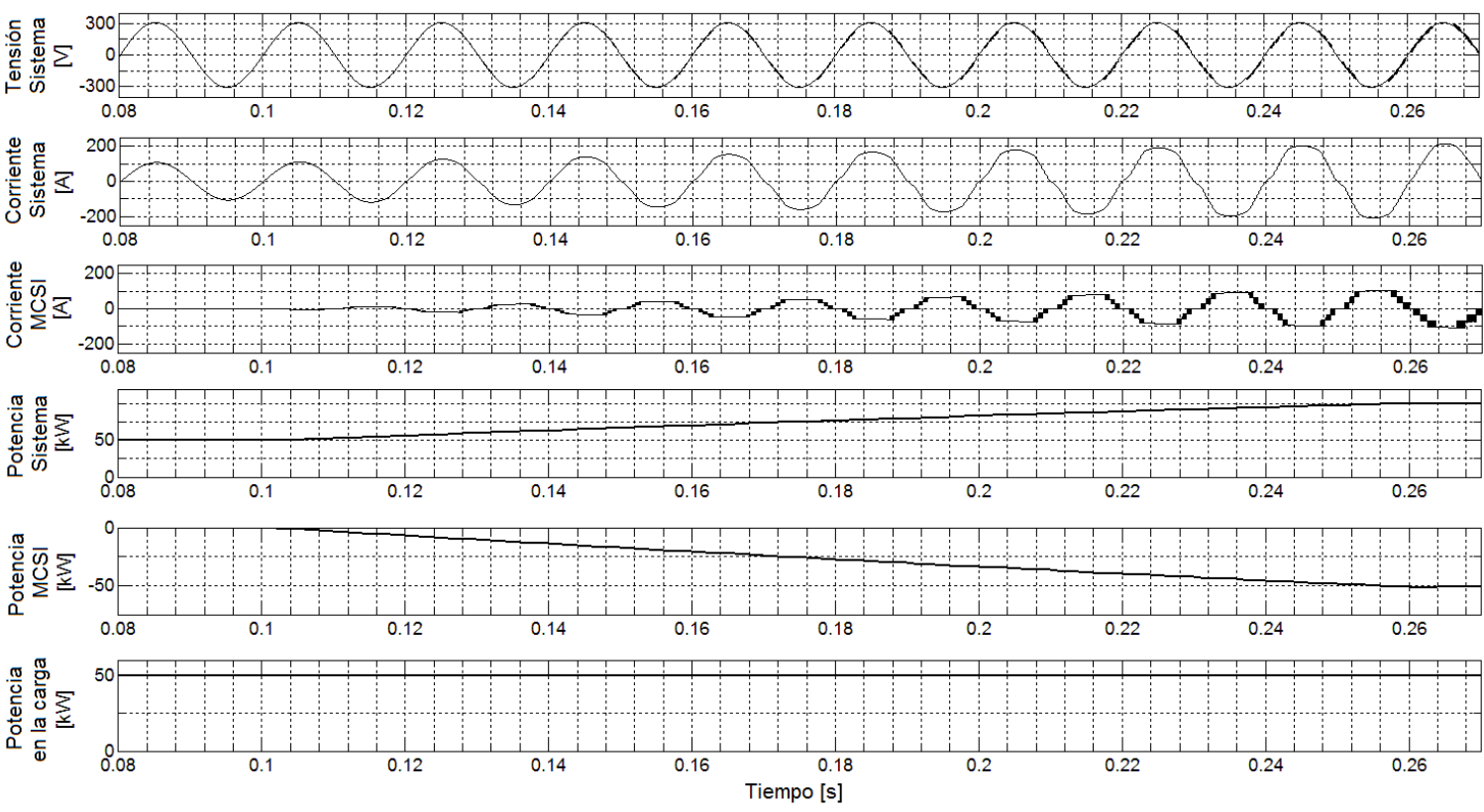

Figura 4.9: análisis de la conexión del electrolizador y el MCSI a la red.
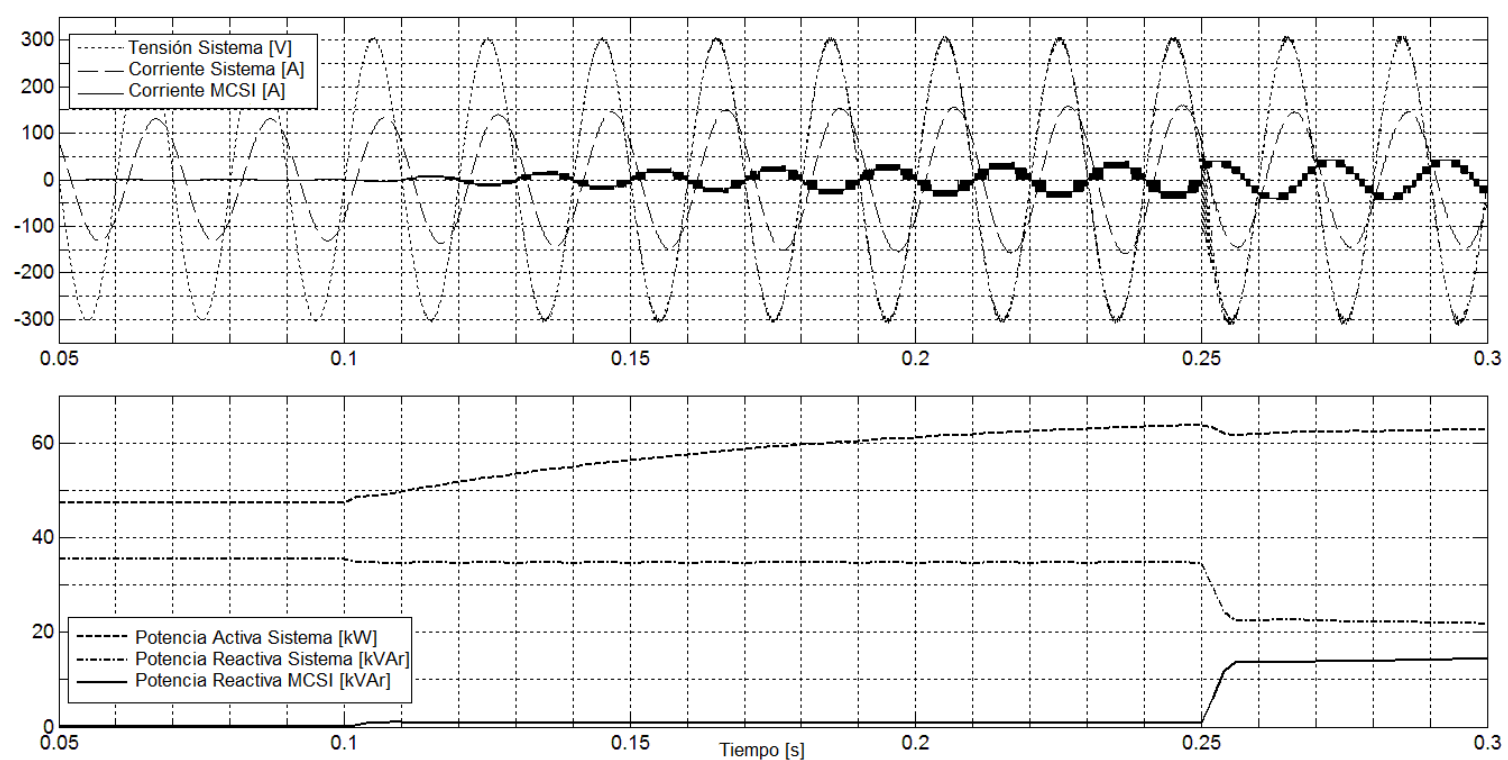

Figura 4.10: conexión del MCSI y el electrolizador al sistema, intercambiando potencia activa y reactiva.

En la Figura 4.11 puede observarse un detalle de las formas de onda resultantes de la conexión de la celda de combustible y el MCSI al sistema eléctrico. En este análisis, los inductores del convertidor son pre-cargados previo a la conexión de la celda al sistema, es por ello que la corriente entregada por el convertidor al sistema toma en forma casi instantánea 
el valor necesario para alimentar a la carga, reduciéndose prácticamente a cero la corriente provista por el sistema. Dado que en este ensayo la carga sólo consume potencia activa, la corriente del convertidor resulta en fase con la tensión del sistema.

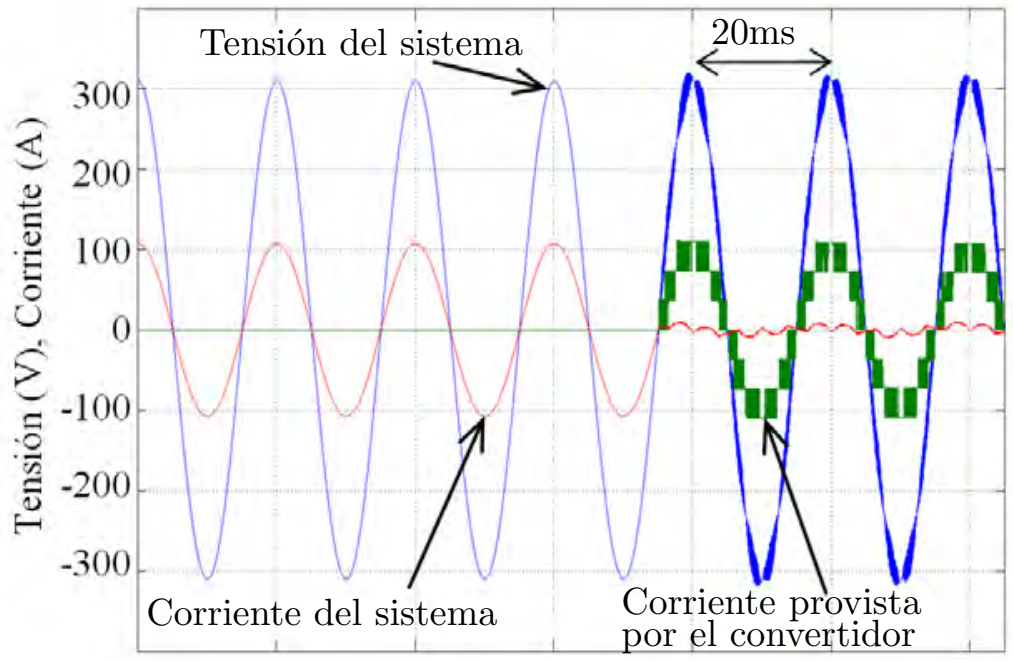

Figura 4.11: formas de onda durante la conexión de la celda de combustible y el MCSI a la red.

En la Figura 4.12 se muestra el flujo de potencia intercambiados por el MCSI y el sistema eléctrico. A los $100 \mathrm{~ms}$ se conecta la celda de combustible a través del MCSI, previamente cargado a su valor nominal, a la red eléctrica. Puede observarse que la potencia activa entregada por el sistema se anula dado que el MCSI está proveyendo a la carga la totalidad de la potencia requerida.
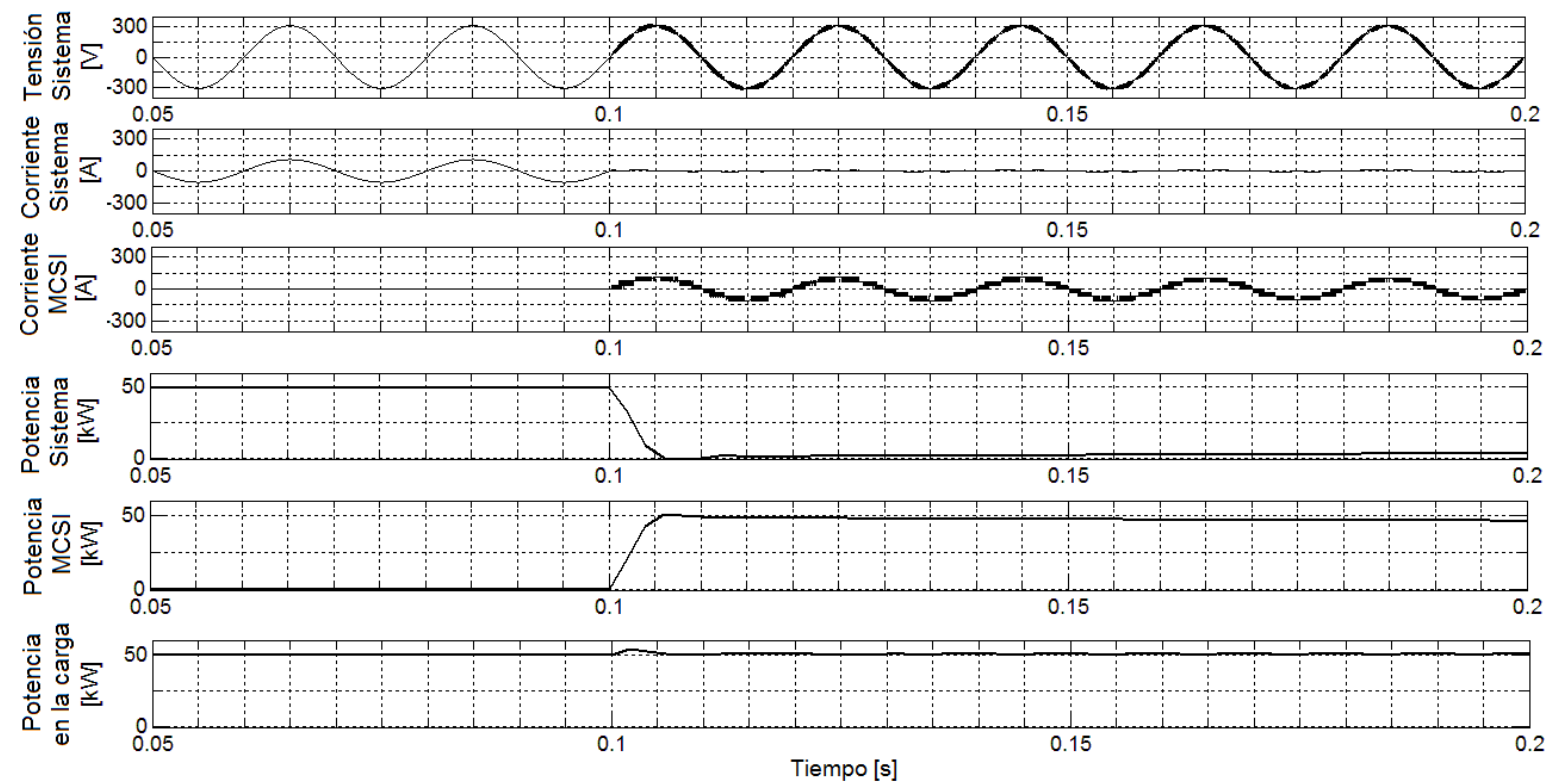

Figura 4.12: análisis de la conexión de la celda de combustible y el MCSI a la red. 
En la Figura 4.13 se muestra el detalle de las corrientes y la tensión del sistema cuando se conecta la celda de combustible y el MCSI a un sistema eléctrico con intercambio de potencia activa y reactiva. En este caso, el convertidor consume energía de la red durante un corto tiempo, aproximadamente medio ciclo, para cargar sus inductores y luego comienza a entregar la energía de la celda de combustible al sistema eléctrico. Se puede observar que la corriente del MCSI está en fase con la tensión y la corriente del sistema se mantiene en el valor dictado por la corriente reactiva consumida por la carga, observándose el desfasaje de $90^{\circ}$ entre la corriente y la tensión del sistema correspondiente a la potencia reactiva pura que suministra el sistema una vez que el convertidor está activo. En este caso particular, el sistema de control está programado para proveer sólo potencia activa, por lo que el sistema eléctrico deberá entregar a la carga la corriente reactiva que se observa luego del arranque del MCSI.

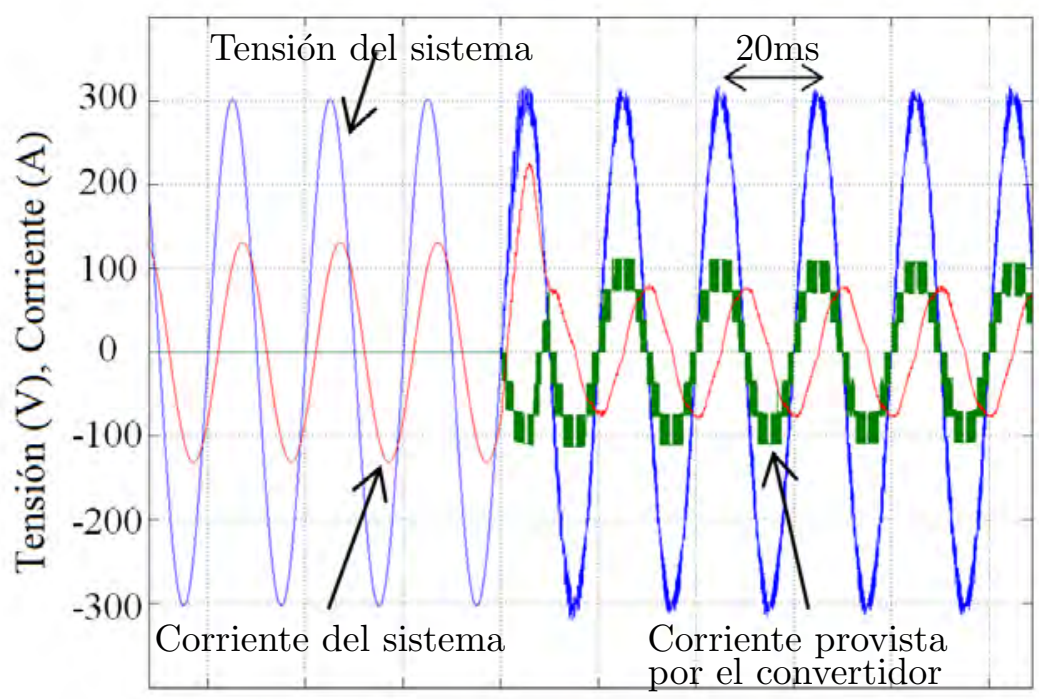

Figura 4.13: conexión del MCSI y la celda de combustible al sistema, intercambiando potencia activa y reactiva.

\subsection{Acondicionamiento de redes eléctricas}

Los MCSI presentan algunas ventajas frente a los MVSI en aplicaciones de alta potencia. Por ejemplo, los inductores son más voluminosos y pesados que los capacitores pero tienen una vida útil más larga y menor tasa de fallas que los capacitores. Pueden soportar alto ripple de tensión sin perder performance, sobrecargas momentáneas de potencia y sus atributos eléctricos no se degradan con el tiempo, con el único requerimiento de mantener una buena disipación del calor generado por las pérdidas, tanto de los conductores como de los núcleos. Esto implica convertidores más seguros, con mayor Tiempo Medio Entre Fallas (MTBF) y menor mantenimiento. Además, los inductores construidos con superconductores de alta temperatura reducirán enormemente las pérdidas, haciendo de los MCSI una opción muy 
eficiente y segura para convertidores de media y alta potencia (Murray et al., 2009). Por otro lado, las topologías multinivel presentan algunas ventajas en lo que respecta a distorsión armónica generada y requerimientos de tensión y corriente en las llaves electrónicas, tanto para fuente de corriente como tensión (Akagi et al., 2007; Barros y Silva, 2008; Rodriguez et al., 2009; Liang et al., 2013).

Aunque los MCSI no han sido ampliamente utilizados todavía, se presentan como una buena opción para mejorar el funcionamiento y la eficiencia en sistemas de distribución eléctrica y aplicaciones industriales donde se requiere alta potencia, o alta corriente (Wang y Ooi, 1993; Liang et al., 2013).

\subsubsection{Sistema Eléctrico Modelo}

Para analizar el funcionamiento del convertidor se utilizará un sistema eléctrico de referencia (Corasaniti et al., 2006), al que se conecta el MCSI en configuración paralelo o "shunt" (Corasaniti et al., 2008; Bhattacharya et al., 2009; Terciyanli et al., 2012). El diagrama unifilar del sistema de distribución de media potencia modelado se muestra en la Figura 4.14. Las cargas de las subestaciones incluidas son básicamente comerciales y residenciales por lo que es más difícil identificar en este caso las fuentes de armónicas, lo que suele ser más simple en el caso de analizar plantas industriales. Es por ello que Corasaniti et al. (2006) realizaron mediciones en campo para obtener datos reales para el modelo.

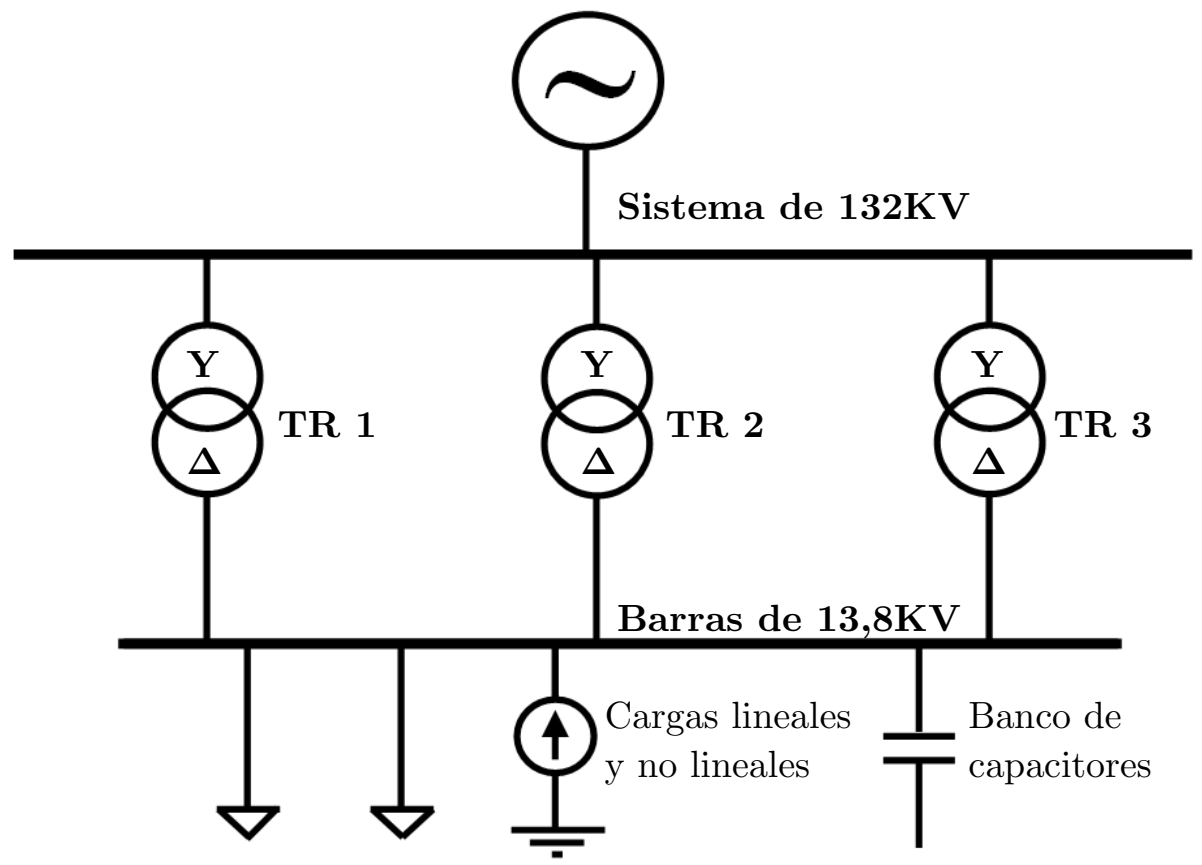

Figura 4.14: sistema eléctrico utilizado como modelo.

El sistema está representado por una fuente ideal de tensión de $132 \mathrm{KV}$ conectada a tres transformadores de características similares $(132 / 34.5 / 13.8 \mathrm{KV}$ y $15 / 10 / 15 \mathrm{MVA})$ y su impedancia equivalente relacionada con la potencia de cortocircuito en $13.8 \mathrm{KV}$. Los 
transformadores están conectados en paralelo en el nivel de $13.8 \mathrm{KV}$, donde están concentradas las cargas. Debido al conexionado $\mathrm{Y} / \Delta$ (estrella/triángulo) de los transformadores no se registran componentes de secuencia cero al nivel de las cargas. Las mediciones realizadas en la red muestran que las cargas no presentan componentes de secuencia negativa en la fundamental. Con estos datos se decide utilizar un modelo balanceado para el sistema en su conjunto (Corasaniti et al., 2006, 2007, 2009). La demanda de potencia activa y reactiva a la frecuencia fundamental se representan mediante un modelo de impedancia constante, mientras que las cargas no lineales, correspondientes a las armónicas presentes en el sistema, se modelan mediante fuentes de corriente sinusoidal. La amplitud y frecuencia de las armónicas modeladas se obtienen de mediciones sobre el sistema. La demanda total de potencia en las barras de $13.8 \mathrm{KV}$ es de 30MVA con un factor de potencia $\cos \varphi=0,8$. Los valores pico de corriente de armónicas y el valor de distorsión total armónica de corriente $\left(\mathrm{THD}_{I}\right)$ medidos se muestran en el Cuadro 4.1. En el Cuadro 4.2 se resumen las tensiones de armónicas y la distorsión total armónica de tensión $\left(\mathrm{THD}_{V}\right)$, se muestran también los límites aceptados por IEEE (1993). Las tensiones de las armónicas $5^{\text {ta }}, 7^{\text {ma }}, 11^{\text {va }}$ y $13^{\text {ra }}$, así como el nivel de $\mathrm{THD}_{V}$, están por encima de los niveles aceptados (Figura 4.15) por lo que es mandatorio realizar la compensación de armónicas propuesta.

Cuadro 4.1: armónicos de corriente.

\begin{tabular}{cccccc}
\hline$i_{h}$ & $i_{5}(\mathrm{~A})$ & $i_{7}(\mathrm{~A})$ & $i_{11}(\mathrm{~A})$ & $i_{13}(\mathrm{~A})$ & $\mathrm{THD}_{I}(\%)$ \\
Valores pico & 81.6 & 58 & 37.2 & 31.4 & 5.45 \\
\hline
\end{tabular}

Cuadro 4.2: armónicos de tensión.

\begin{tabular}{ccc}
\hline $\begin{array}{c}\text { Tensiones } \\
\text { Armónicas }\end{array}$ & $\begin{array}{c}100 \% \\
\text { Carga }\end{array}$ & $\begin{array}{c}\text { Límite } \\
\text { IEEE }\end{array}$ \\
\hline V5 (\%) & 3,07 & 3 \\
V7 (\%) & 3,04 & 3 \\
V11 (\%) & 3,05 & 3 \\
V13 (\%) & 3,04 & 3 \\
THDV $(\%)$ & 6,10 & 5 \\
\hline
\end{tabular}

\subsubsection{Filtro Activo}

Los MCSI Simétricos son una excelente alternativa para la implementación de filtros activos de mediana y alta potencia, debido a su alta confiabilidad, su operación tolerante a fallas, a que no utiliza grandes capacitores y que no requiere el agregado de inductores de acoplamiento a la red, mejorando su respuesta dinámica. Los objetivos del Filtro Activo aplicado al sistema bajo estudio son: a) reducir el contenido armónico en barras de 13,8KV por debajo de los límites aprobados por la IEEE, medido a plena carga, y b) obtener un factor de potencia superior a 0,96. Esta última condición permite mantener el nivel de potencia del 


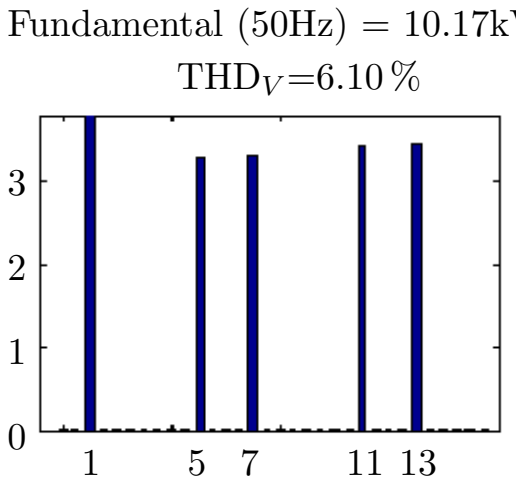

(a) Tensión del sistema ( $\%$ de la fundamental)
Fundamental $(50 \mathrm{~Hz})=1652 \mathrm{~A}$

$\mathrm{THD}_{I}=5.45 \%$

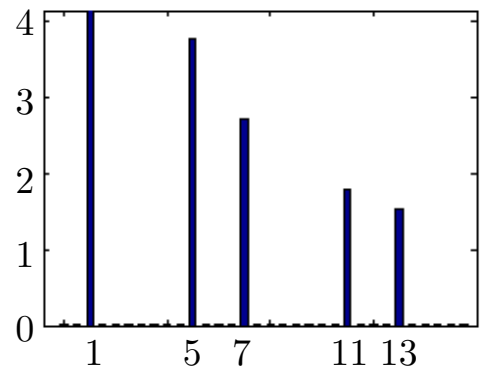

(b) Corriente del sistema ( $\%$ de la fundamental)

Figura 4.15: espectros del sistema sin compensación: fundamental y primeras cuatro armónicas.

MCSI dentro de valores razonables, ya que $\cos \phi=1$ demandaría demasiada potencia sin ofrecer una ventaja equivalente al sistema eléctrico. En la Figura 4.16 se analiza el diagrama esquemático del conexionado del MCSI a la red eléctrica y las señales de control asociadas, los parámetros del convertidor se resumen en el Cuadro 4.3

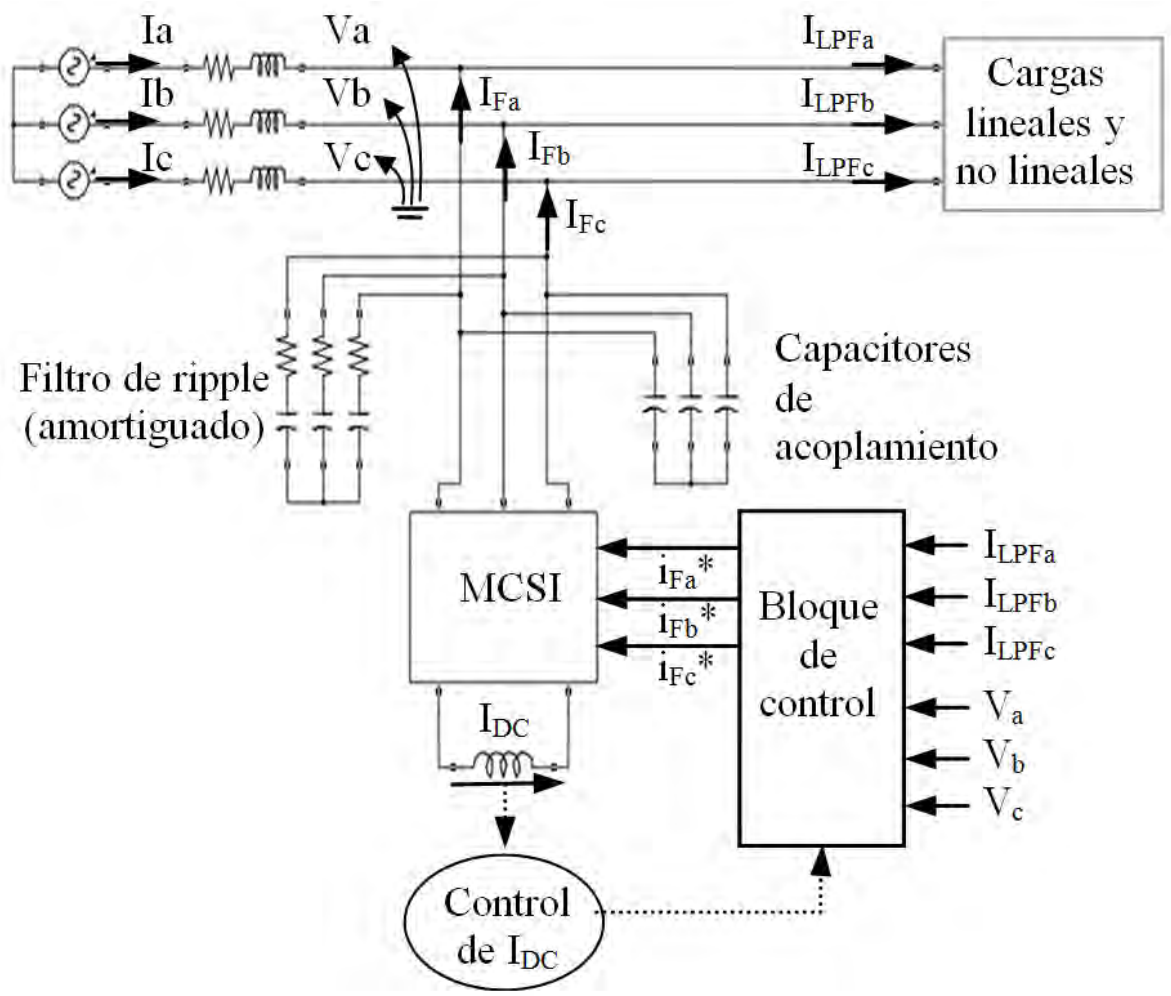

Figura 4.16: diagrama esquemático del sistema con el filtro activo y las señales de control.

Se utilizan dos bancos de componentes pasivos debido a que: a) en toda aplicación con convertidores en redes eléctricas se recomienda el agregado de un filtro $R C$ amortiguado (Corasaniti et al., 2009) para reducir las componentes de alta frecuencia debidas a la 
Cuadro 4.3: parámetros del MCSI.

\begin{tabular}{lrc}
\hline Parámetro & Valor & \\
\hline Inductores Principales & 250 & $\mathrm{mH}$ \\
Corriente principal $\left(I_{D C}\right)$ & 900 & $\mathrm{~A}$ \\
Inductores Divisores & 200 & $\mathrm{mH}$ \\
Frecuencia del sistema & 50 & $\mathrm{~Hz}$ \\
Capacitores de acople & 5 & $\mu \mathrm{F}$ \\
Filtro de ripple & 3 & $\mu \mathrm{F}$ \\
& 30 & $\Omega$ \\
Frecuencia de conmutación & 4065 & $\mathrm{~Hz}$ \\
Frecuencia de ripple en la salida & 12195 & $\mathrm{~Hz}$ \\
\hline
\end{tabular}

conmutación tanto en tensión como en corriente, b) un banco de capacitores de bajo valor permite el acoplamiento del MCSI a la red suavizando los sobrepicos de tensión que pudieran generarse debido a la conmutación de corriente (Aguirre et al., 2010a). La fuente principal de CC del convertidor consiste en un inductor de valor apropiado, cuya corriente puede ser regulada intercambiando potencia activa con el sistema, mediante la componente $\overline{I d}$.

El Bloque de Control de la Figura 4.16 produce las señales de referencia que serán utilizadas en la modulación del MCSI para la generación de las señales de disparo de las llaves. Su detalle se muestra en la Figura 4.17, donde se aprecian sus componentes principales en forma esquemática. Dado que el control se basa en la transformación de coordenadas "abc $\rightarrow d q 0 "$ (4.3) (Akagi et al., 2007; Aguirre et al., 2010a) es indispensable contar con un sistema de sincronismo con la red, para ello se miden las tensiones del sistema a fin de obtener el ángulo de referencia de fase $\theta$ mediante un PLL trifásico. El ángulo de sincronismo obtenido se utiliza en (4.3) para transformar las corrientes consumidas por la carga $\left(I_{L P F a}\right.$, $I_{L P F b}$ y $\left.I_{L P F c}\right)$ en sus componentes $I d$ e $I q$.

$$
\begin{aligned}
& {\left[\begin{array}{c}
I_{d} \\
I_{q}
\end{array}\right]=\frac{2}{3}\left[\begin{array}{ccc}
\sin \theta & \sin \left(\theta-\frac{2 \pi}{3}\right) & \sin \left(\theta+\frac{2 \pi}{3}\right) \\
\cos \theta & \cos \left(\theta-\frac{2 \pi}{3}\right) & \cos \left(\theta+\frac{2 \pi}{3}\right)
\end{array}\right]\left[\begin{array}{l}
I_{L F P a}^{*} \\
I_{L F P b}^{*} \\
I_{L F P c}^{*}
\end{array}\right]} \\
& {\left[\begin{array}{c}
I_{F a}^{*} \\
I_{F b}^{*} \\
I_{F c}^{*}
\end{array}\right]=\left[\begin{array}{cc}
\sin \left(\theta-\frac{\pi}{6}\right) & \cos \left(\theta-\frac{\pi}{6}\right) \\
\sin \left(\theta-\frac{2 \pi}{3}-\frac{\pi}{6}\right) & \cos \left(\theta-\frac{2 \pi}{3}-\frac{\pi}{6}\right) \\
\sin \left(\theta+\frac{2 \pi}{3}-\frac{\pi}{6}\right) & \cos \left(\theta+\frac{2 \pi}{3}-\frac{\pi}{6}\right)
\end{array}\right]\left[\begin{array}{c}
I_{d}^{*} \\
I_{q}^{*}
\end{array}\right]}
\end{aligned}
$$

Mediante dos filtros Pasa-Bajos de segundo orden se separan y procesan las componentes media y armónicas de la corriente de la carga, en coordenadas " $d q "$ : 
- $\overline{I d}$ - es la componente media de $I d$ y está relacionada directamente con la potencia activa que provee el sistema a la carga. Dado que en esta aplicación se utilizará al MCSI únicamente como filtro activo, esta componente no se incluirá en la modulación porque se espera que la potencia activa de la carga sea provista por el sistema eléctrico. En caso de disponerse de alguna fuente que pueda proveer energía al inductor principal del convertidor es posible utilizar a $\overline{I d}$ como referencia de la potencia requerida por la carga.

- $\overline{I q}$ - es la componente media de $I q$, relacionada con el factor de potencia según su definición tradicional. Se utiliza en la modulación del convertidor para permitir inyectar potencia reactiva a la red y asi compensar el factor de potencia de la carga. Dado que el MCSI tiene una capacidad limitada de corriente, se limita la cantidad de potencia reactiva generada para permanecer dentro de los límites de operación segura del convertidor.

- $\widetilde{I d}, \widetilde{I q}$ - son las componentes armónicas de $I d$ e $I q$ respectivamente. Están directamente relacionadas con las perturbaciones y armónicas presentes en la red, por lo que se inyectan directamente a la modulación del convertidor. De esta manera el MCSI genera las corrientes armónicas que la carga requiere, eliminando estas perturbaciones del sistema y mejorando asi la calidad del suministro eléctrico.

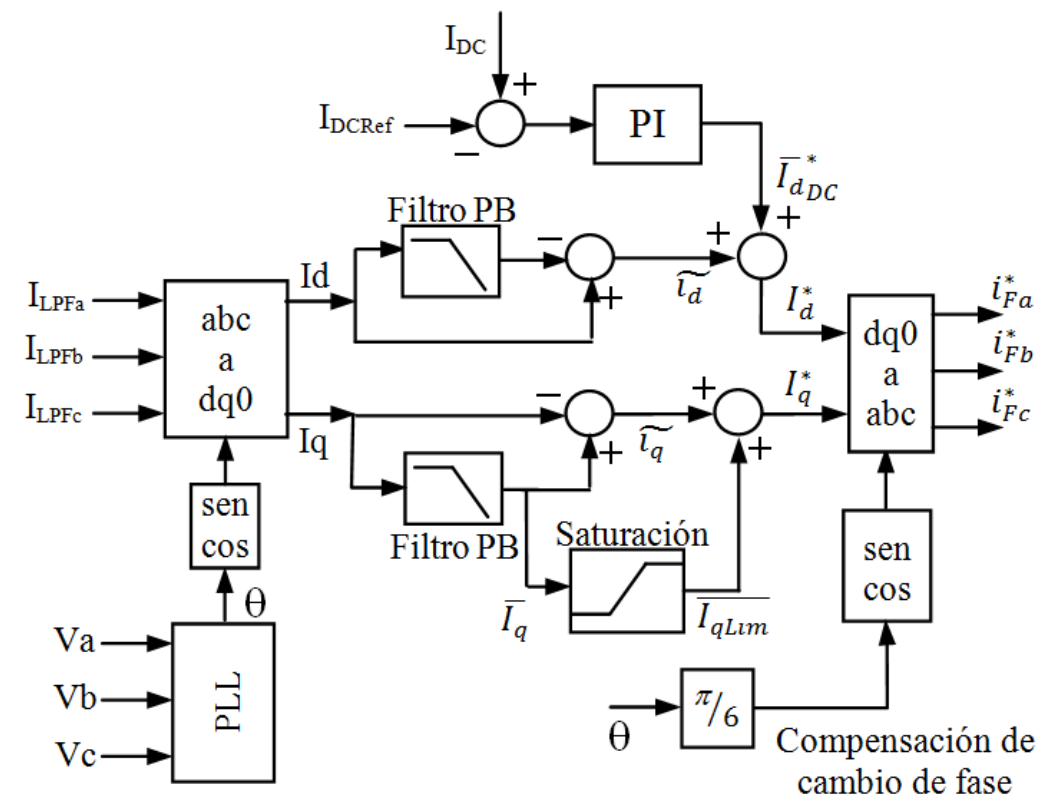

Figura 4.17: diagrama esquemático del bloque de control.

La corriente del inductor principal del MCSI $\left(I_{D C}\right)$ puede ser modificada mediante el intercambio de potencia activa con el sistema eléctrico, debiendo tomarse siempre una pequeña cantidad de potencia para compensar las pérdidas. Para mantener constante su valor, se mide y compara con un valor de referencia $\left(I_{D C R e f}\right)$, obteniendo una señal de error $\left(\overline{I_{d_{D C}}^{*}}\right)$ que se aplica a la entrada de un controlador PI para modificar el valor medio de la 
corriente $I d(\overline{I d})$ que el MCSI inyecta a la red y regular así el intercambio de potencia activa del convertidor en forma semejante al control de tensión en el bus de continua de un MVSI. Sumando la componente armónica de potencia activa $\widetilde{I d}$, para eliminar esas perturbaciones del sistema, se obtiene la señal de referencia $I_{d}^{*}$.

La segunda señal de referencia $I_{q}^{*}$ se genera sumando la componente armónica $\widetilde{I q}$ con la referencia de potencia reactiva a inyectar al sistema $\overline{I q L i m}$, que es igual a $\overline{I q}$ pero limitada a un valor máximo para evitar que el convertidor pueda saturar o entrar en sobremodulación, lo que generaría distorsiones y armónicas indeseadas en la red. Utilizando la antitransformación $" d q 0 \rightarrow a b c "$ (4.4) se obtienen las referencias $I_{F a}^{*}, I_{F b}^{*}$ y $I_{F c}^{*}$, las que se ingresan directamente a la modulación PSC-SPWM, sustituyendo al bloque de señales sinusoidales de la Figura 3.35. Para realizar la transformación al dominio del tiempo es necesario ajustar el valor del ángulo de referencia de fase $\theta$ agregándole un offset de $\frac{\pi}{6}$ debido al cambio de fase entre las referencias de la modulación y la salida real del convertidor causado por la modulación Tri-Logic descripta en la Sección 3.3.1.

En la Figura 4.18 se muestran las formas de onda de tensión y corriente presentes en el sistema al momento de la conexión del MCSI, puede observarse que durante el transitorio de arranque del convertidor, entre $0,1 \mathrm{~s}$ y $0,15 \mathrm{~s}$, no se presentan perturbaciones debido al crecimiento gradual de las corrientes, comandadas por el bloque de control. La corriente del sistema se acerca en fase a la tensión por el efecto de la inyección de potencia reactiva y esto produce además un ligero aumento de la tensión. Se observa también que aparecen en la tensión las distorsiones de alta frecuencia típicas de los convertidores electrónicos, pero en la Figura 4.19 se demuestra que las armónicas resultantes están por debajo de los límites establecidos por IEEE (1993). Para mensurar la influencia del convertidor en el cálculo de la distorsión armónica total, tanto $\mathrm{THD}_{V}$ como $\mathrm{THD}_{I}$, se consideran las armónicas presentes hasta los $50 \mathrm{KHz}$. De esta manera se están teniendo en cuenta también las interferencias causadas por las conmutaciones del convertidor. La $\mathrm{THD}_{V}$ se reduce a $1,90 \%$ cuando se considera sólo hasta la armónica 50, como especifican las normas IEEE, mientras que no se aprecian cambios en la $\mathrm{THD}_{I}$. Puede notarse que la frecuencia efectiva de conmutación del convertidor es de $12.195 \mathrm{~Hz}$, tres veces la frecuencia de modulación de cada módulo, $4065 \mathrm{~Hz}$, debido al funcionamiento multinivel del conjunto y a la modulación PSC-SPWM.

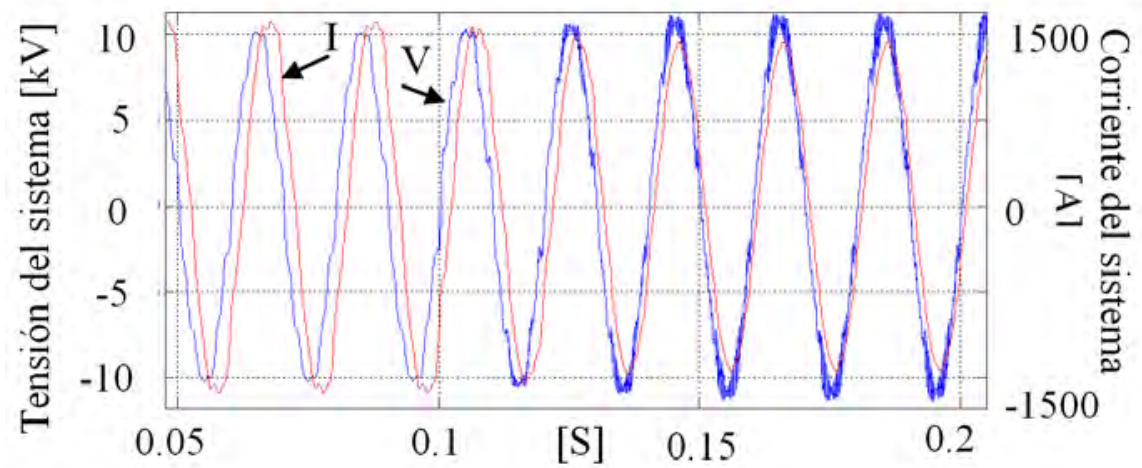

Figura 4.18: tensión y corriente del sistema eléctrico durante el transitorio de arranque del MCSI. 


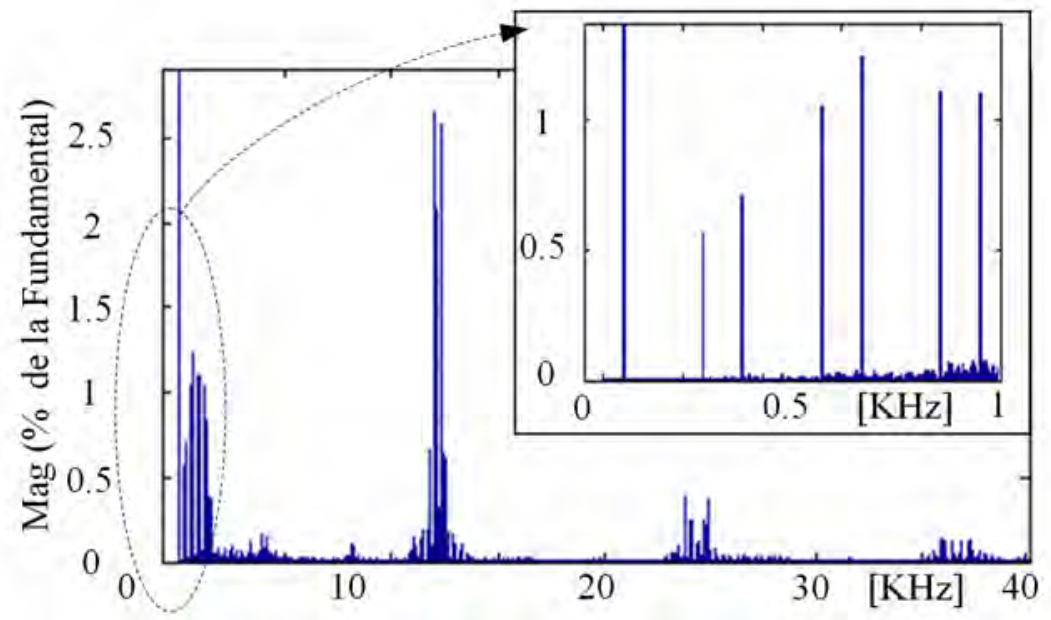

Figura 4.19: espectro de la tensión del sistema con la compensación activa. Fundamental $=$ $10.78 \mathrm{KV}, \mathrm{THD}_{V}=3.10 \%$.

La acción del MCSI sobre las armónicas de corriente se muestran en la Figura 4.20, donde se observa una marcada reducción frente a los valores originales del Cuadro 4.1, obteniéndose una $\mathrm{THD}_{I}$ de sólo $1,83 \%$.

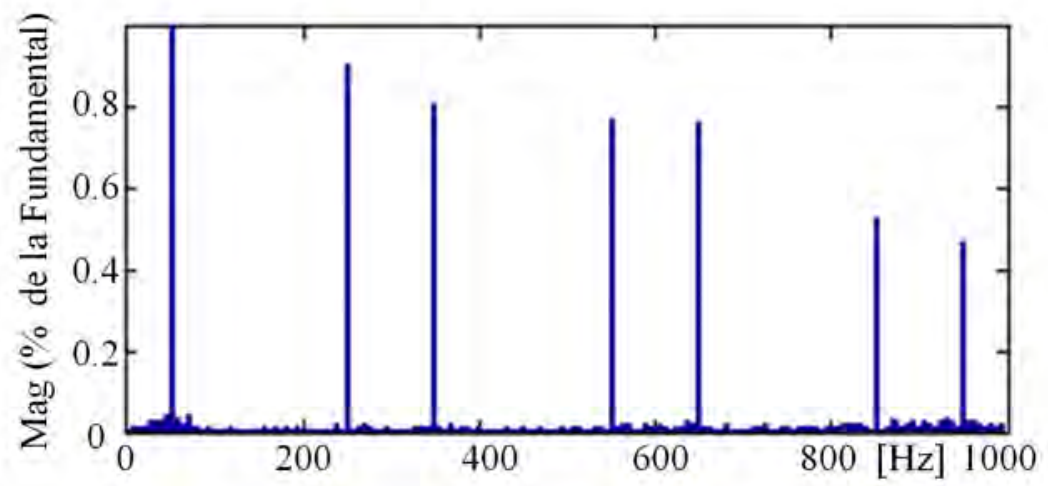

Figura 4.20: espectro de la corriente del sistema con la compensación activa. Fundamental $=$ $1422 \mathrm{~A}, \mathrm{THD}_{I}=1.83 \%$.

Para verificar la respuesta dinámica del sistema frente a variaciones de carga se realiza el ensayo que se muestra en la Figura 4.21, donde se puede observar cómo la corriente del sistema disminuye a los $0,4 \mathrm{~s}$ sin que se produzcan distorsiones ni oscilaciones. La respuesta dinámica del MCSI se adapta perfectamente al sistema eléctrico analizado.

\subsubsection{Compensación de factor de potencia}

En la Figura 4.22 se muestran las potencias, activa y reactiva, suministradas por el sistema y el convertidor en cuatro situaciones típicas: 


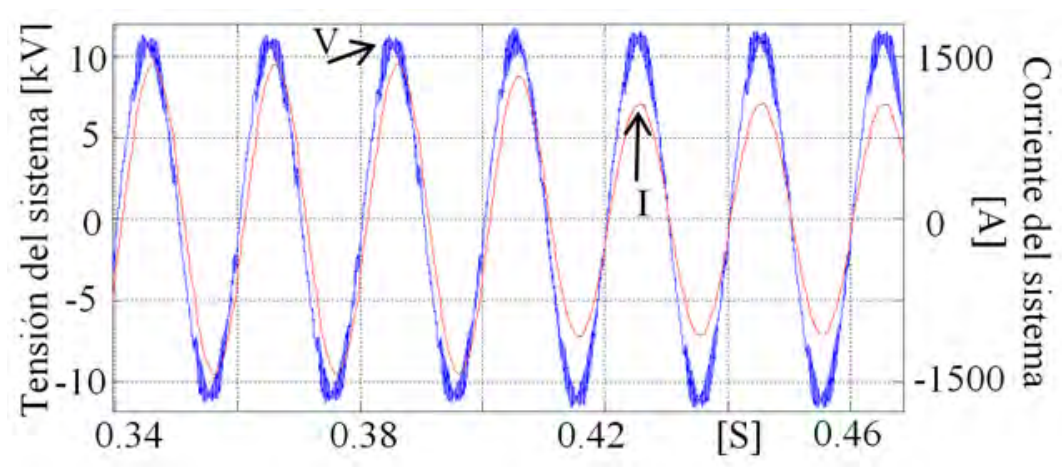

Figura 4.21: tensión y corriente del sistema eléctrico durante el transitorio de cambio de carga al $75 \%$.

Cuadro 4.4: tensiones Armónicas, resultados y verificaciones con compensación.

\begin{tabular}{cccc}
\hline Tensiones armónicas & Carga 100\% & Carga $75 \%$ & Límites IEEE \\
\hline V5 (\%) & 0.56 & 0.49 & 3 \\
V7 (\%) & 0.70 & 0.64 & 3 \\
V11 ( \%) & 1.05 & 0.93 & 3 \\
V13 ( \%) & 1.24 & 1.03 & 3 \\
THDV ( \%) & 3.10 & 2.43 & 5 \\
\hline
\end{tabular}

- Entre 0 y 0,1s el MCSI está desconectado y se tienen las potencias del sistema original.

- Entre 0,10 s y 0,15 s se realiza el arranque controlado del convertidor, a fin de minimizar las perturbaciones en la red. La potencia reactiva crece linealmente a medida que los inductores del MCSI se cargan hasta que se llega a su condición de trabajo nominal.

- Entre 0,15s y 0,40s el MCSI opera compensado tanto armónicas y distorsiones como también inyectando potencia reactiva, corrigiendo asi el factor de potencia. A plena carga el convertidor le entrega al sistema un valor medio de $\mathrm{Q}_{C O N V}=11.8 \mathrm{MVAr} \mathrm{y}$, como la carga necesita $\mathrm{Q}_{C A R G A}=17.8 \mathrm{MVAr}$, el sistema sólo debe proveer $\mathrm{Q}_{\text {SISTEMA }}$ $=6$ MVAr. Como la potencia activa que provee el sistema a plena carga es de $22.2 \mathrm{MW}$ el factor de potencia resultante es $\cos \phi=0.965$. Como la carga está representada por un modelo paralelo de impedancia constante, sus requerimientos de potencia varían con el cuadrado de la tensión aplicada, es por ello que cuando el convertidor compensa potencia reactiva y la tensión aumenta, también aumentan la potencia activa y reactiva consumidas por la carga. La compensación de 11,8MVAr a plena carga produce un aumento del $5,7 \%$ en la tensión de las barras de $13,8 \mathrm{KV}$ y por consiguiente un aumento de 1,9MVAr en la potencia reactiva consumida por la carga.

- A los 0,4s se produce una reducción abrupta en la carga al 75\%, luego del transitorio propio del sistema el conjunto se estabiliza en un nuevo punto de trabajo. Como el sistema de control está programado para que el MCSI entregue la máxima potencia 
reactiva posible (11.8MVAr), el factor de potencia resultante al reducir la carga es de $0,99 \%$, quedando la corriente prácticamente en fase con la tensión, como se observa en la Figura 4.21. En caso que la carga requiera menos potencia reactiva, la regulación sobre la corriente $I_{q}$ descripta en la Figura 4.17 modificará el punto de trabajo del convertidor a fin de entregar sólo lo que la carga requiera, evitando sobrecompensar al sistema eléctrico.

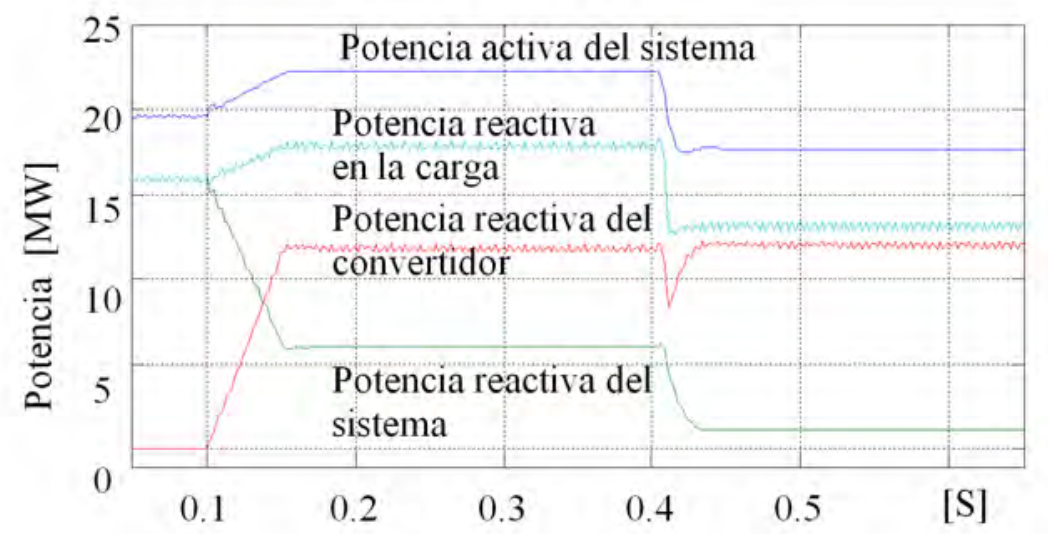

Figura 4.22: acción del convertidor sobre el sistema. Arranque a los $0,1 \mathrm{~s}$ y cambio a $75 \%$ de carga a los $0,4 \mathrm{~s}$.

La forma de onda de corriente generada por el convertidor, antes de pasar por el filtro de ripple, se muestra en la Figura 4.23, donde se pueden observar claramente los siete niveles. La acción combinada del filtro amortiguado de ripple y de los capacitores de conexión eliminan las componentes de alta frecuencia resultando en una corriente inyectada al sistema como se muestra en la Figura 4.23(b). 


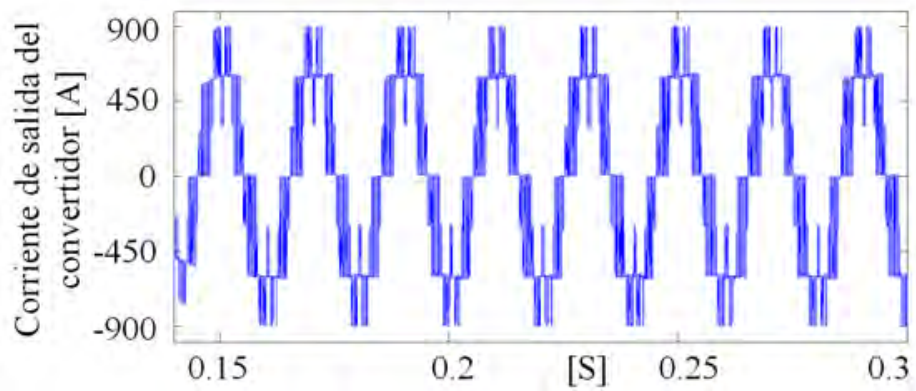

(a) corriente de salida del filtro activo.

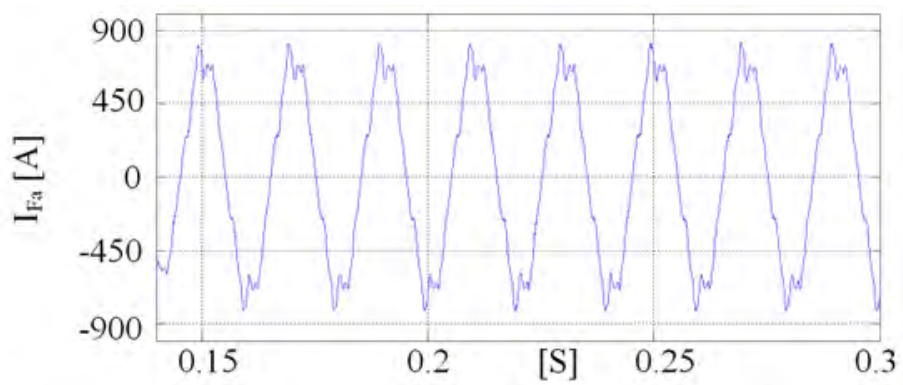

(b) corriente inyectada el sistema.

Figura 4.23: corriente generada por el MCSI, a) sin filtrar, b) corriente filtrada que ingresa al sistema.

\subsection{Conclusiones}

De las simulaciones y análisis realizados en este capítulo se concluye que los convertidores MCSI Simétricos son una excelente opción para su utilización en aplicaciones de sistemas eléctricos y como interfaz con energías alternativas. Su rápida respuesta dinámica permite no sólo el intercambio de potencias activa y reactiva con baja distorsión, sino también la implementación de filtros activos con posibilidad de compensar distorsiones y armónicas de alta frecuencia. Además, su rango dinámico permite adaptarse a distintos puntos de funcionamiento, característica indispensable para adaptarse a las cambiantes condiciones de las energías alternativas. 


\section{Capítulo 5}

\section{Conclusiones}

\subsection{Conclusiones}

Las diferentes tecnologías de convertidores electrónicos de potencia multinivel están siendo utilizadas cada vez más en aplicaciones industriales y de sistemas eléctricos, debido principalmente a la mayor confiabilidad y prestaciones, pero también a los requerimientos cada vez más exigentes de las aplicaciones en sí. En especial los convertidores multinivel fuente de corriente presentan grandes ventajas en aplicaciones de alta corriente, ya que en conjunto el convertidor puede operar por encima de la capacidad de corriente de sus componentes individuales. Sin embargo, el knowhow disponible sobre estas topologías todavía requiere cierta profundización para la producción de equipos de uso industrial.

Además, la confiabilidad de los convertidores de alta potencia resulta vital, por un lado para garantizar la operación de las aplicaciones en los que se utilizan, sobre todo en sistemas eléctricos; y por otro lado por la cantidad de energía involucrada, que en caso de fallas puede generar daños físicos considerables tanto a equipos como a personas. Es por ello que el desarrollo de convertidores y técnicas de operación tolerantes a fallas es vital para un uso seguro en aplicaciones de altas potencias.

En esta tesis se realizó el estudio de la topología MCSI Simétrico, o Multi Rating Inductor $M C S I$, y sus aplicaciones a sistemas eléctricos y energías alternativas. Se comenzó por el principio de funcionamiento, las técnicas de modulación y el control de balance, llegando a obtener un conjunto de conocimientos mínimos para la simulación de un convertidor en aplicaciones reales y la verificación de los principios básicos de funcionamiento en un prototipo a escala.

El estudio detallado de la topología y sus aplicaciones permitieron realizar aportes significativos en los siguientes temas:

Modulación: Se aplicó la combinación de dos técnicas de modulación, PSC-SPWM y 
Tri-Logic PWM, al convertidor bajo estudio, verificando que las señales de disparo generadas producen un adecuado funcionamiento, baja distorsión de salida y el balance natural de las corrientes internas. La combinación resultante presenta una excelente respuesta dinámica, produciendo un régimen de trabajo uniforme entre todas las llaves del convertidor, distribuyendo equitativamente la carga entre las llaves evitando así puntos críticos de confiabilidad. Además, el efecto se mantiene independientemente del punto de trabajo $\left(m_{a}\right)$, por lo que a bajas corrientes de salida se siguen activando todas las llaves, produciendo la misma cantidad de conmutaciones por ciclo pero sólo entre los niveles más bajos de corriente. Esto permite obtener la misma frecuencia equivalente de conmutación en la carga, facilitando el filtrado y simplificando el estudio de las emisiones electromagnéticas (EMI) resultantes. Como resultado se obtiene una lógica de simulación compacta, con posibilidad de procesamiento en paralelo y fácil de implementar en una FPGA con mínimos retardos.

Balance natural: Se verificó que el desplazamiento de las portadoras de la modulación PSC-SPWM produce un balance natural de las corrientes internas del convertidor, que si bien no es robusto frente a perturbaciones, variaciones de los componentes del convertidor y tiene un pequeño error sistemático en estado estacionario, su efecto es lo suficientemente fuerte como para permitir el estudio de la topología básica, tanto en las simulaciones como a nivel prototipo, sin la necesidad de complejas estrategias de control que pudieran enmascarar efectos propios del circuito o la conmutación de las llaves.

Minimización de la frecuencia de conmutación: Se demostró que es posible hacer uso de la redundancia de combinaciones de llaves que generan estados de cero corriente que minimicen el número de conmutaciones por ciclo de las llaves. Para ello se diseñó una máquina secuencial lógica que detecta la secuencia de conmutación activa del convertidor y reemplaza el estado cero generado por la modulación por aquél que evite que una de las llaves conmute. De esta manera se consigue reducir en aproximadamente 1/3 la cantidad de conmutaciones que debe realizar una llave, lográndose además un esquema de conmutación donde la llave sólo conmuta durante la mitad de un ciclo de la frecuencia de salida. Minimizar la cantidad de conmutaciones de las llaves permite escalar los resultados, de simulación y experimentales, a convertidores de mucha mayor potencia, además permite aumentar la vida útil de las llaves y sus circuitos auxiliares, reduciendo los requerimientos de potencia de los circuitos de disparo.

Minimización de las pérdidas por conmutación: Se logró reducir drásticamente la potencia disipada en las llaves durante las conmutaciones mediante un adecuado diseño del conexionado interno de los módulos. Se comprobó que la minimización de ciertas inductancias parásitas de los conexionados permite un intercambio libre de corriente entre los dos IGBT que conmutan en una secuencia, esto conlleva a una especie de conmutación "suave" sin disipación de potencia en ellos. Los resultados de este análisis fueron verificados mediante simulaciones detalladas en Matlab Simulink y PSPICE, y corroboradas en el prototipo experimental donde no se requiere de disipador para funcionar en régimen permanente, entregando a la carga una potencia de 3KW. Gene- 
ralizando este resultado se concluye que es necesario analizar en detalle cada elemento parásito presente en la construcción normal de un convertidor MCSI, dado que sus efectos son muy diferentes a los que se producen en un MVSI. La suma de los efectos de conmutación suave y de la reducción del número de conmutaciones por ciclo de las llaves permite escalar los resultados de simulación a prototipos de alta potencia, como los utilizados en las aplicaciones presentadas en esta tesis.

Tolerancia a fallas: La topología estudiada en esta tesis puede garantizar el funcionamiento continuo a pesar de una falla, mediante la conexión de un módulo de respaldo en caliente (hotspare) que reemplaza en forma activa al módulo fallado, permitiendo el reemplazo de la parte dañada sin detener la operación del convertidor. Mediante simulaciones se demostró que la estructura de protección diseñada permite la conexión del módulo de respaldo sin afectar notoriamente la salida. Además, la modularidad de la topología permite ciclar los módulos en operación para la realización de tareas de mantenimiento preventivo y reducir el stock de piezas de repuesto, minimizando los costos de operación y mantenimiento. Estas características únicas hacen de la topología Single Rating Inductor MCSI una opción excelente para su utilización en sistemas eléctricos y en general para todas aquellas aplicaciones que requieran de alta disponibilidad y MTBF.

Control del balance de corrientes: Se presentaron dos técnicas para el control del valor medio de las corrientes internas y garantizar así el balance de corrientes del convertidor. Los dos métodos se basan en aplicar ligeras modificaciones en la modulación, de manera de modificar gradualmente las corrientes de los distintos módulos sin afectar sustancialmente las formas de ondas de salida. El método de control por cambio de amplitud de las portadoras se muestra a primera vista como más atractivo, ya que posee tres entradas de control independientes: la amplitud de la portadora triangular de cada módulo. Pero éste método tiene ganancia variable con el punto de trabajo del convertidor y por lo tanto su aplicación es más efectiva para valores altos de $m_{a}$. Ésto implica un control de ganancia variable, que si bien no es difícil de implementar en la práctica, complica el control general del convertidor. Por otro lado, a valores bajos de $m_{a}$, el control por variación de fase de las portadoras triangulares presenta mejor respuesta, pero sólo se disponen de dos entradas de control, ya que la fase relativa entre las tres portadoras debe sumar siempre $360^{\circ}$. Ambos métodos permiten implementar un control muy sencillo, cuya mayor complejidad es la digitalización de los controladores PI internos y por lo tanto puede ser programados en la FPGA, para trabajar en forma independiente y paralela con la lógica de simulación, sin que interfieran entre si sus tiempos de procesamiento.

Respuesta dinámica: Tanto en los resultados de simulación como en los experimentales se puede observar que la topología estudiada presenta una excelente respuesta dinámica, obteniéndose una corriente de salida sin distorsiones ni sobrepicos tanto para adaptarse a cambios abruptos del punto de trabajo como a saltos de carga. Esta característica es indispensable para su utilización en aplicaciones con condiciones de trabajo variables, como en la interfaz con energías alternativas, así como aquellas en las que se requiere una buena respuesta en frecuencia, como en un filtro activo en el que se requiera compensar 
o eliminar armónicas altas.

Aplicaciones: La suma de las características propias de la topología permite su utilización en aplicaciones industriales y de sistemas eléctricos. De las simulaciones realizadas se obtiene una excelente respuesta, bajo ripple de salida con pequeñas capacidades de acoplamiento, buen rango dinámico y un control sencillo. Se demostró que es posible utilizar un convertidor MCSI Simétrico para realizar un filtro activo y como interfaz con energías alternativas. En ambos casos se obtiene una solución con características de funcionamiento totalmente comparables con los MVSI, que sumadas a su alta confiabilidad tanto por la topología en si como por la alta confiabilidad de los inductores en comparación con los capacitores de potencia hacen de la topología una excelente opción para las aplicaciones seleccionadas.

Construcción de un prototipo: Para validar los conceptos teóricos básicos mediante resultados experimentales se construyó un prototipo real de un convertidor trifásico, controlado por medio de una FPGA en donde se implementaron las lógicas de modulación y control. Los resultados experimentales obtenidos son comparables a los de simulación, verificando el diseño y los análisis realizados. Gracias al correcto diseño del conexionado interno de los módulos, la potencia disipada por las llaves es completamente despreciable, incluso trabajando en estado estacionario a plena carga. La implementación de las lógicas de simulación y control en una FPGA permiten su funcionamiento en forma independiente, a alta velocidad y con la precisión individual que cada cálculo requiere, contando además con la mayor confiabilidad que la tecnología de lógica programable presenta frente a los microprocesadores y DSP. El prototipo de $3 \mathrm{KW}$ construido permitirá profundizar los resultados obtenidos en esta tesis a fin de aumentar el knowhow sobre esta topología con los sucesivos trabajos de investigación.

Como conclusión final se puede observar que, dada la tecnología actual de llaves y técnicas de control, no existen impedimentos para la realización de MCSI de potencia, los que presentan un excelente comportamiento y ventajas considerables frente a los MVSI. Por lo tanto es indispensable mejorar el knowhow disponible y el desarrollo de nuevas topologías, para aprovechar las ventajas particulares de estos convertidores en aquellas aplicaciones en las que sobresalen por sus especiales características.

\subsection{Trabajos a futuro}

Se pretende que esta tesis sea el punto de partida para el desarrollo de la tecnología de convertidores fuente de corriente multinivel, por lo cual se plantean las siguientes líneas de investigación:

Sincronización del prototipo con la red eléctrica: se está trabajando en la implementación de los algoritmos de sincronización y control de flujo de potencia en la misma FPGA donde se realiza la modulación. Esto supone la realización de un módulo que, basado en un PLL y en las transformaciones $a b c \rightarrow \alpha \beta 0 \rightarrow d q 0$, brinde información en 
tiempo real del ángulo de fase de la red a fin de sincronizar la generación de corriente y permitir el control del intercambio de potencia, tanto activa como reactiva, del convertidor con el sistema eléctrico al que está conectado (Hoffmann et al., 2011). La ventaja de utilizar una FPGA es que las operaciones necesarias para la sincronización pueden realizarse en forma paralela con el resto del control y la modulación, minimizando los errores por demoras o desfasajes en los cálculos(Aguirre et al., 2013d).

Construcción de un modelo de sistema eléctrico: a fin de obtener resultados experimentales del conexionado y funcionamiento del MCSI en un sistema real se está trabajando en el diseño de un modelo de sistema eléctrico a escala, que incluya los accionamientos y protecciones necesarios para una operación segura y la realización de pruebas como la generación de armónicas, desbalances, huecos y sobrepicos de tensión, entre otras.

Desarrollo de métodos de modulación: si bien la modulación aplicada en esta tesis tiene un funcionamiento adecuado, es posible hacer uso de la enorme capacidad de cálculo disponible en las FPGA actuales a fin de aprovechar la redundancia de combinaciones de llaves para generar cada nivel de corriente de salida, e investigar otras técnicas de modulación que permitan una generación más eficiente de las señales de disparo y un balance de corriente más robusto frente a perturbaciones. Un posible camino es utilizar una combinación de modulación vectorial con algoritmos de selección del estado siguiente basados en funciones de peso para realizar un control de balance de mayor velocidad de respuesta, desarrollados específicamente para la topología.

Modelo de sistema híbrido: dado que los convertidores pueden considerarse como una sucesión de sistemas lineales conmutados, con el agregado de una lógica para la modulación, es posible estudiar el conjunto en forma global como un sistema híbrido y por lo tanto aplicar estrategias de control no lineal. Sería deseable analizar en una primera etapa el balance de corrientes en los inductores divisores mediante funciones de energía de Lyapunov o similares, para luego extender el análisis al funcionamiento dinámico del convertidor para garantizar su estabilidad en todo el rango dinámico de operación.

Ampliar el rango de aplicaciones: las especiales características de la topología le brindan una gran versatilidad, lo que permite investigar su adaptación a otras aplicaciones, entre ellas:

- rectificadores de alta potencia, baja distorsión y factor de potencia variable,

- interfaz con sistemas de generación eólica y solar,

- filtros activos de conexión serie,

- control de máquinas eléctricas, motores y generadores.

Análisis de otras topologías MCSI: se puede extender el análisis y los resultados obtenidos para investigar las características de otras topologías MCSI, como la "Multi Rating Inductor MCSI", entre otras. La extensión del knowhow a otras topologías permitirá la divulgación de las posibilidades de los MCSI en otras aplicaciones, produciendo un efecto multiplicativo en publicaciones y libros. 


\section{Apéndice A}

\section{Diseño del prototipo}

A fin de verificar mediante resultados experimentales los resultados de simulación, se construyó un prototipo de convertidor MCSI Simétrico de tres módulos, con la posibilidad de generar siete niveles en la corriente de salida. En la Figura A.1 se puede observar el esquema general del prototipo, que consta de las siguientes partes principales:

- Fuente de corriente principal

- Banco de capacitores de acoplamiento

- Circuitos de disparo

- Control por FPGA

- Carga trifásica variable

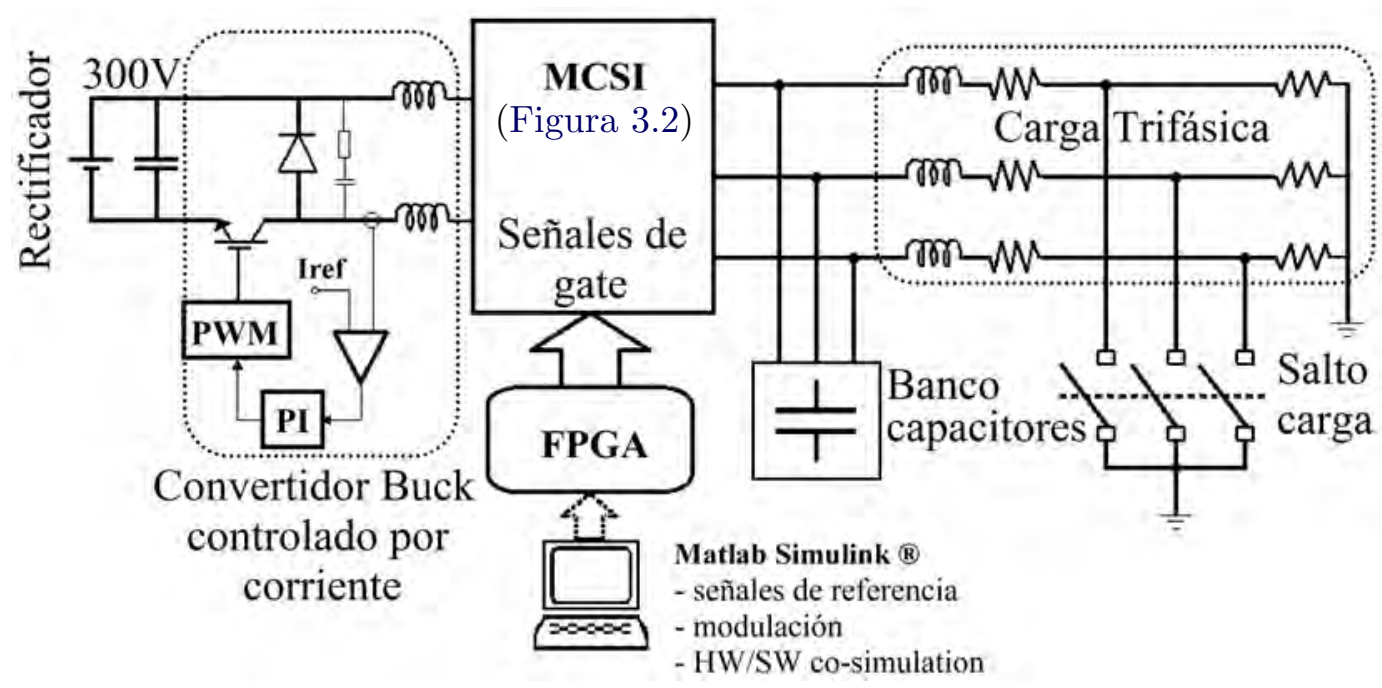

Figura A.1: esquema general del prototipo. 
$\mathrm{Al}$ prototipo básico se le agregó un cuarto módulo para generar nueve niveles y permitir el funcionamiento tolerante a fallas. En el Cuadro A.1 se resumen los principales componentes y características.

Cuadro A.1: datos constructivos del prototipo experimental.

\begin{tabular}{llll}
\hline Potencia de salida & $P=3 K V A$ & $P=0-3 K W$ & $Q=0-3 K V A R$ \\
Salida & $I_{M A X}=10 A$ & $V_{M A X}=450 \mathrm{~V}$ & 7 Niveles \\
Carga & $R_{L}=8-16 \Omega$ & $L_{L}=10 \mathrm{mH}$ & $P=3 K W$ \\
Inductores principales & $L=2 x 120 \mathrm{mH}$ & $R_{D C}=0,8 \Omega$ & $I_{M A X}=10 \mathrm{~A}$ \\
Inductores divisores & $L=80 \mathrm{mH}$ & $R_{D C}=0,6 \Omega$ & $I_{M A X}=4 A$ \\
Banco de capacitores & $C=9 x 0,47 \mu F$ & & \\
Llaves: IRG4PC40UD & $V_{M A X}=600 \mathrm{~V}$ & $I_{M A X}=20 \mathrm{~A}$ & Encapsulado TO-247 \\
Diodos: RHRP1560 & $V_{M A X}=600 \mathrm{~V}$ & $I_{M A X}=15 \mathrm{~A}$ & Encapsulado TO-220 \\
\hline
\end{tabular}

\section{A.1. Fuente de corriente principal}

La fuente principal debe proporcionar una corriente constante, regulada y con mínimo ripple, a partir de la fuente de energía con la que sea alimentado el convertidor. Para transformar en corriente constante a la alimentación de tensión proveniente de un rectificador, se utiliza una configuración reductora, o Buck, regulada por ancho de pulso (PWM). La corriente de los inductores de la fuente se mide mediante un sensor integrado de efecto Hall tipo ACS710, cuya salida está aislada galvánicamente de la medición con un valor base (offset) de $2,5 \mathrm{~V}$ y fondo de escala en $5 \mathrm{~V}$. La señal obtenida se filtra para obtener el valor medio y se compara con una fracción de la tensión de referencia de $5 \mathrm{~V}$ del circuito integrado modulador de ancho de pulso SG3526. La alta ganancia de lazo del circuito modulador de ancho de pulso se debe principalmente a la ganancia del amplificador de error, del tipo transconductancia, en cuya salida se coloca el controlador PI que consiste en un circuito RC serie conectado entre el pin 3 del integrado y masa. A fin de obtener el mayor rango dinámico en la regulación, es necesario un ciclo de trabajo $D$ con la máxima excursión posible, por ello se utiliza la suma de las dos salidas del SG3526, unidas mediante diodos. El disparo del IGBT de control se hace a través de un circuito opto-aislado del tipo FOD3180, el cual puede entregar en su salida hasta $2 \mathrm{~A}$, lo que permite tiempos de conmutación lo suficientemente rápidos para minimizar las pérdidas en la llave. La topología reductora se completa con un diodo ultra-rápido del tipo RHRP1560. El circuito impreso (PCB) debe realizarse de manera de minimizar los lazos de corriente para reducir los efectos de EMI y aumentar el rendimiento general del convertidor (Figura A.2). 


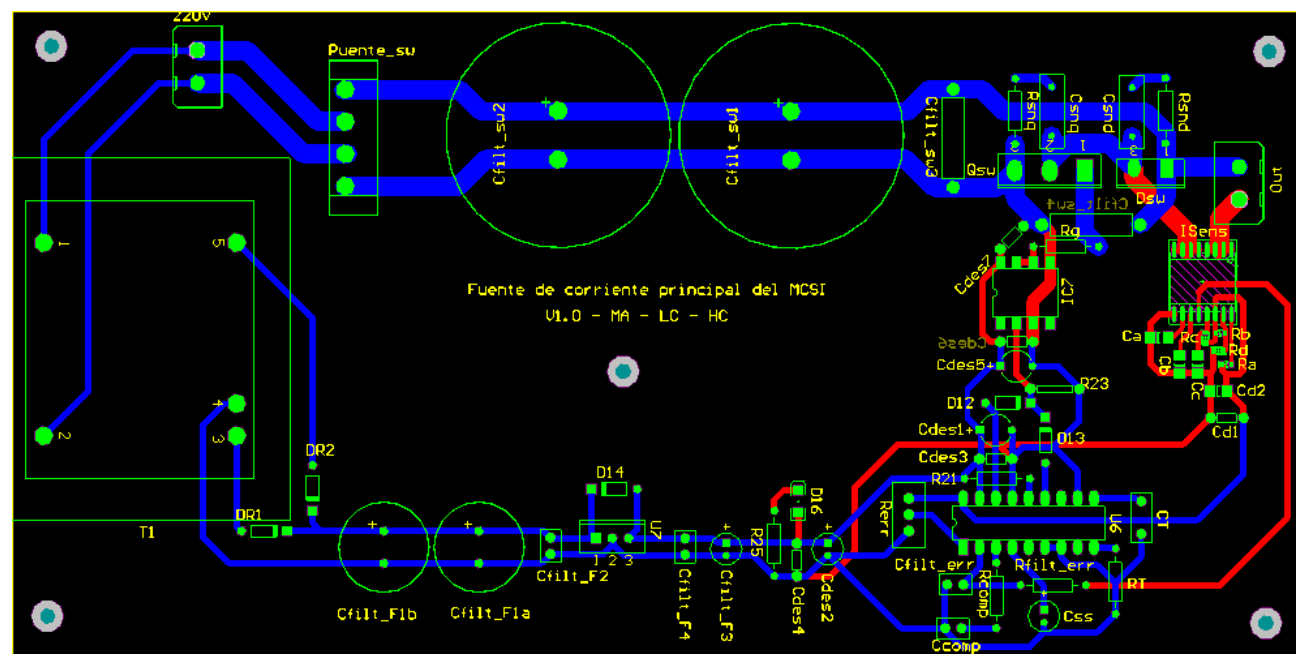

Figura A.2: circuito impreso de la fuente principal de corriente.

\section{A.2. Banco de capacitores}

Todo convertidor fuente de corriente necesita capacitores de acoplamiento para poder controlar cargas inductivas, ya que es preciso suavizar los saltos de corriente en las conmutaciones para evitar sobretensiones causadas por las inductancias de la carga y los cableados. La capacidad requerida depende de la corriente que se debe conmutar, la frecuencia de trabajo y el ripple de tensión aceptable en la salida. Para poder conmutar una corriente máxima de $4 \mathrm{~A}$, valor máximo de diseño de cada módulo, en el tiempo de conmutación de las llaves, $1 \mu \mathrm{s}$, es suficiente con un banco de capacitores de sólo $0,47 \mu \mathrm{F}$ para garantizar un ripple de tensión inferior a 10V. Si bien la función principal del banco de capacitores es evitar sobretensiones causadas por la parte inductiva de la carga, todos los cableados intermedios producirán también sobretensiones en los momentos de conmutación, debido a las inductancias parásitas. Es por ello que se distribuye el banco de capacitores, colocando una parte en cada módulo y ubicando los componentes lo más cerca posible del punto de conexión de las llaves con la carga, de esta manera se consigue minimizar las sobretensiones que deben soportar las llaves, pudiendo operarse hasta 500V con las llaves de 600V (IRG4PC40UD) utilizadas. Distribuyendo el banco de capacitores en los puntos de conexionado, se puede utilizar capacitores de menor valor y mejorar la capacidad de corriente de ripple de estos componentes. Además, esta construcción mejora la modularización del convertidor permitiendo el reemplazo, reparación o mantenimiento de los capacitores junto con el módulo asociado.

\section{A.3. Circuitos de disparo}

El circuito de disparo de cada llave se ha diseñado de forma de ser lo más simple y rápido posible, para ello se utiliza un circuito integrado optoacoplado especialmente diseñado para la tarea, del tipo FOD3180, que puede proveer una corriente superior a 2A para manejar 
la compuerta de la llave IGBT. Como se muestra en la Figura A.3 cada llave tiene su propia fuente aislada a fin de evitar interferencias entre las señales de las distintas llaves y también para garantizar una simetría que permita igualar los tiempos de disparo y retardo. Las alimentaciones aisladas se obtienen a partir de un convertidor indirecto o flyback (Mohan et al., 2005), cuyo transformador consta de ocho arrollamientos: un primario, un secundario de $+5 \mathrm{~V}$ para alimentación de la lógica y seis secundarios de $+15 \mathrm{~V}$ para la alimentación de las llaves del módulo. Está realizado sobre un núcleo toroidal para minimizar el acoplamiento capacitivo entre bobinados y asi evitar que los niveles de alimentación y referencia de las llaves varíen cuando se producen cambios bruscos $(d v / d t)$ en la tensión de emisor de los IGBT. El primario se construye dividido en dos partes para minimizar el efecto proximidad y además distribuir mejor el flujo magnético, mejorando el acoplamiento magnético a pesar del gran entrehierro realizado para evitar la saturación del núcleo, de esta manera se minimizan las diferencias entre las tensiones de los bobinados secundarios causadas por el flujo disperso del transformador.

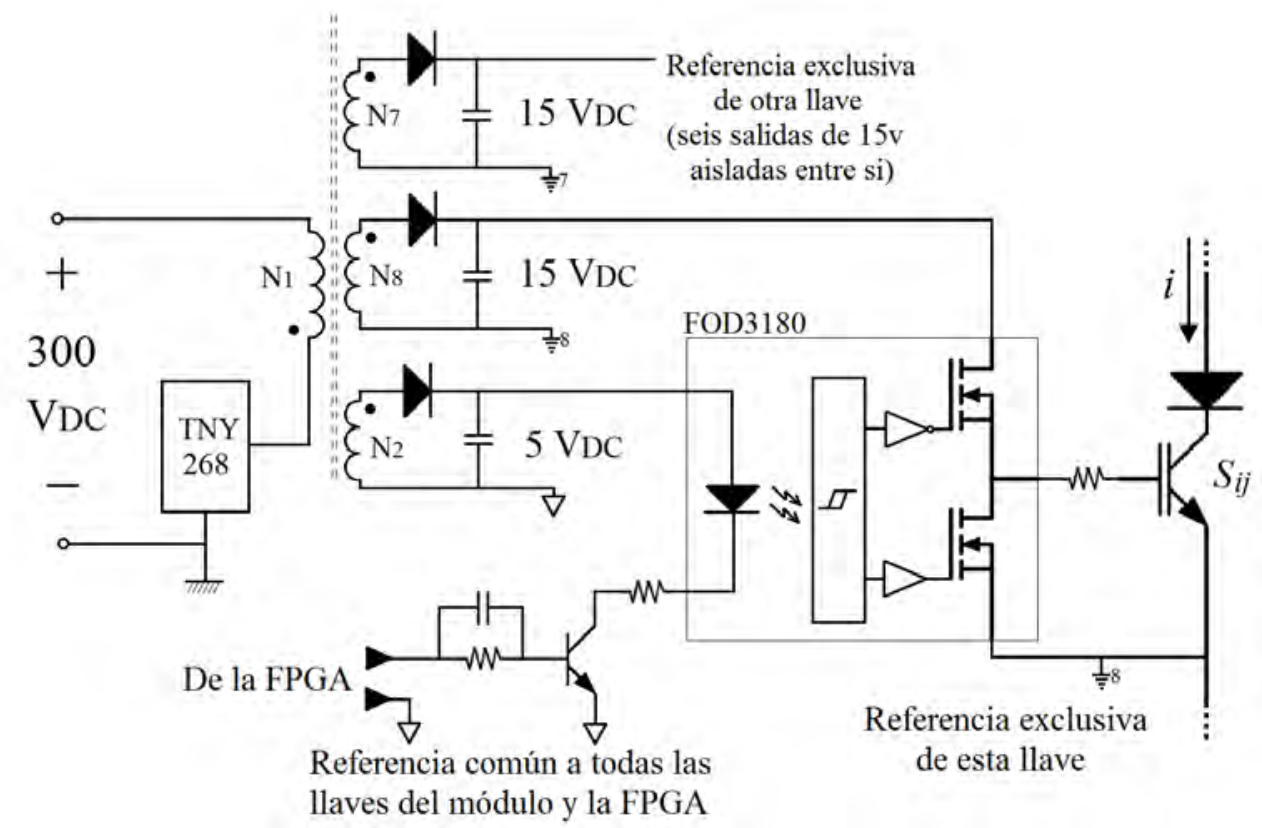

Figura A.3: diagrama esquemático del circuito de disparo de una llave.

El control del convertidor flyback se construye a partir de un circuito integrado de la familia TNY268 (Powerint, 2009) que incluye todos los elementos necesarios para su funcionamiento: circuito de auto-arranque, control PWM y llave de potencia, por lo cual permite reducir el tamaño y costo del sistema. La realimentación de las tensiones de salida se realiza mediante un opto-acoplador estándar trabajando en el punto de mayor ganancia a fin de minimizar el error en las salidas, un zener en serie con el led del opto-acoplador permite fijar con buena precisión la tensión de estado estacionario de los secundarios. En los convertidores flyback la energia presente en el núcleo del transformador, cuando la llave corta la alimentación al primario, se reparte entre los secundarios. Este principio básico 
de funcionamiento produce un efecto de regulación compartida entre los secundarios del transformador, ya que aquel que requiere más carga tendrá menos tensión en su capacitor de salida y por lo tanto será el primero en recibir carga del núcleo. Este efecto produce al cabo de varios ciclos que todos los capacitores de salida terminen con la la misma fracción de la tensión nominal. Esto permite que el control realimentado, que sólo puede monitorear directamente a uno de los secundarios, pueda reconocer la variación de carga en alguna de las salidas no supervisadas y actuar en consecuencia, produciendo una regulación aproximada de los otros secundarios. En las Figuras A.4 y A.5 pueden observarse las formas de onda de tensión de compuerta (gate) de las llaves 1 a 4 de un módulo del prototipo, obtenidas experimentalmente. La Figura A.4(a) muestra un ciclo completo de encendido y apagado donde se puede apreciar que no existen oscilaciones y sobrepicos que compliquen el funcionamiento de las llaves, aunque existen pequeñas diferencias en los valores finales de alimentación de las distintas llaves, debidos a la dispersión de campo en entre bobinados del transformador y que además las alimentaciones carecen de regulación fuera de la propia del convertidor flyback. La tensión de alimentación del circuito de disparo de cada llave se ha fijado en aproximadamente 14V para evitar llegar en algún momento a los 20V máximo de ruptura de los IGBT utilizados. Si bien esta tensión varia ligeramente entre llaves, esas variaciones prácticamente no afectan a los tiempos de conmutación.

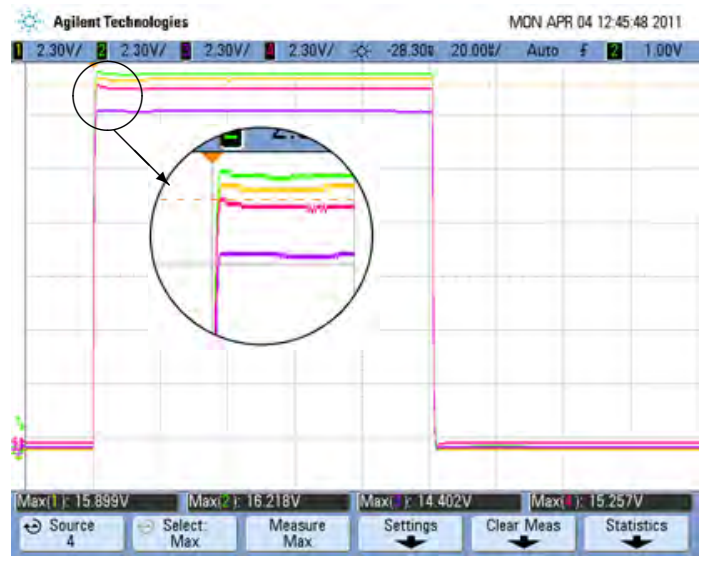

(a) un ciclo completo, no se presentan sobrepicos ni oscilaciones. $(2,3 \mathrm{~V} / \mathrm{div}, 20 \mu \mathrm{s} / \mathrm{div})$

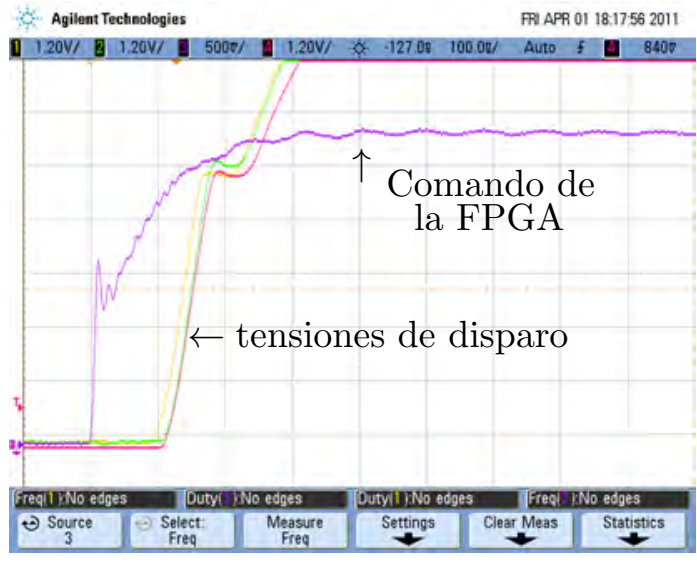

(b) retardo entre la señal de comando de la FPGA y la tensión resultante de disparo, se observa una mínima dispersión en las tensiones de compuerta. (100ns/div)

Figura A.4: tensiones de disparo de compuerta (Gate) de los IGBT de un módulo.

En la Figura A.4(b) se puede observar el retardo entre una señal de disparo de la FPGA y la respuesta del circuito en las llaves 1 a 3. Se aprecia un retardo de aproximadamente 100ns al comienzo de la transición y que la pendiente de las señales de compuerta es menor, debido a la carga que ejercen las capacidades internas del IGBT sobre el circuito de disparo. No se aprecia una diferencia considerable entre las señales de disparo de las tres llaves, esto es debido al cuidadoso diseño del circuito.

En la Figura A.5(a) se puede observar un detalle del transitorio de encendido de las llaves, 
con la forma de onda típica del disparo de un IGBT. El tiempo de subida de la corriente de colector $\left(t_{r i}\right)$ de las distintas señales es prácticamente el mismo, aproximadamente 40ns, y está definido por el tiempo que tarda la tensión de compuerta en pasar de la tensión de activación $\left(V_{G S t h}\right)$ a la tensión de plena corriente $\left(V_{G S I o}\right)$. El tiempo de bajada de la tensión colector-emisor $\left(t_{f v}\right)$ está definido por el tiempo que tarda la llave en descargar la capacidad intrínseca entre la compuerta (Gate) y el colector, siendo la tensión de compuerta constante durante esta parte de la transición, como se puede apreciar en la Figura A.5(a). Los tiempos de bajada de la tensión observados son de aproximadamente 60ns, con muy poca dispersión entre llaves. Por último, la Figura A.5(b) muestra en detalle el transitorio de apagado de las llaves, si bien la caída de tensión de compuerta se produce en aproximadamente 500ns, por el efecto de "tailing" (Mohan et al., 2005) el apagado real del IGBT se produce en algo menos que $1 \mu \mathrm{s}$, permitiendo la conmutación suave referida en la Sección 3.4.

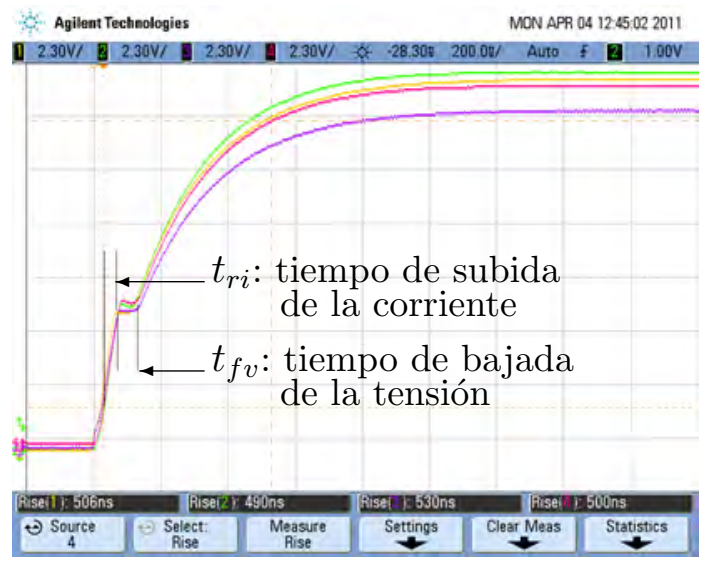

(a) encendido. (200ns/div)

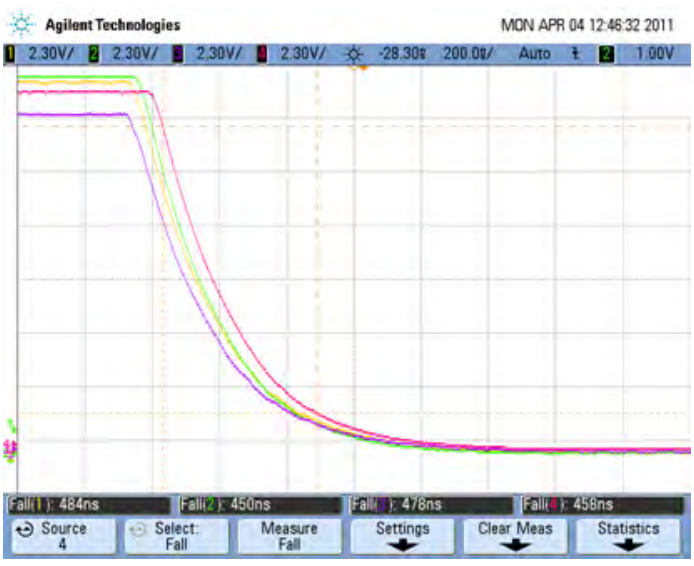

(b) apagado. (200ns/div)

Figura A.5: formas de onda de las tensiones de disparo de compuerta (Gate) de los IGBT de un módulo.

Si bien existen esquemas circuitales más complejos para el disparo de IGBT de potencia, que incluyen monitoreo de tensión, detección y alerta de fallas y encendido y/o apagado dinámico, se busca en estos prototipos minimizar la influencia de la electrónica auxiliar (analógica y digital) para poder centrar el estudio en el funcionamiento propio del convertidor de potencia. El uso de IGBT de alta potencia en un equipo industrial real implica la utilización de circuitos de disparo que, en general, están aislados ópticamente por lo que se puede adaptar directamente el control por FPGA realizado para este prototipo.

En la Figura A.6 se muestra el circuito impreso de un módulo, que consta de la fuente de alimentación, los transistores para adaptación de nivel desde la FPGA, la pistas de interconexión interna y el soporte físico para los IGBT y los diodos de las llaves. Puede notarse su construcción modular, la distribución lo más simétrica posible de los componentes y las islas utilizadas para minimizar la inductancia entre las llaves. 


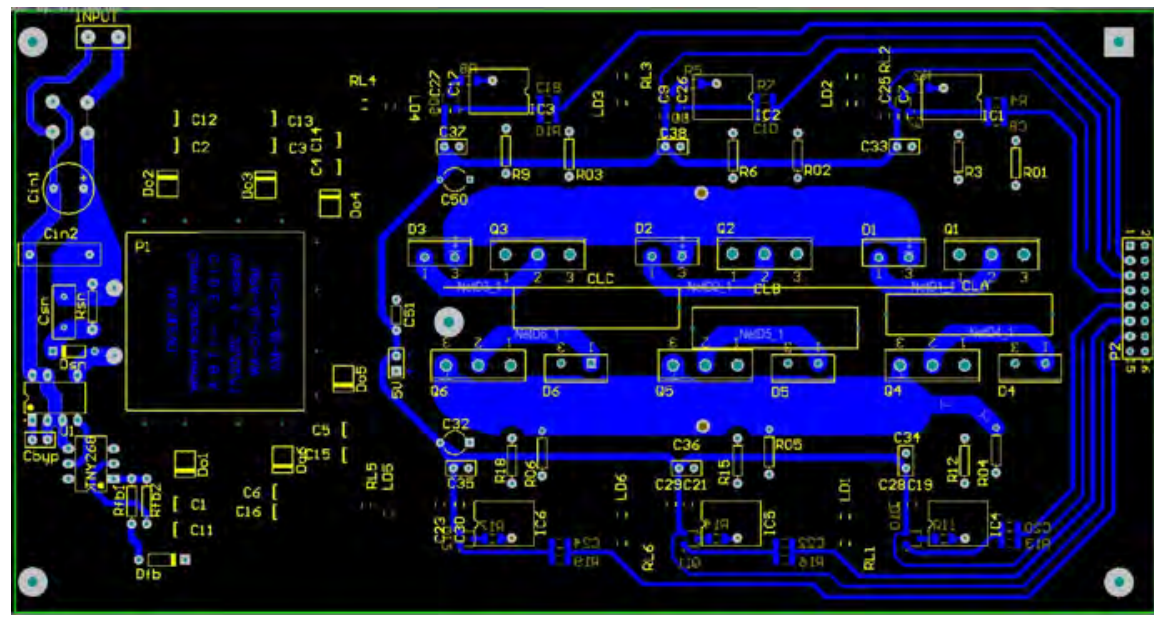

(a) Cara inferior

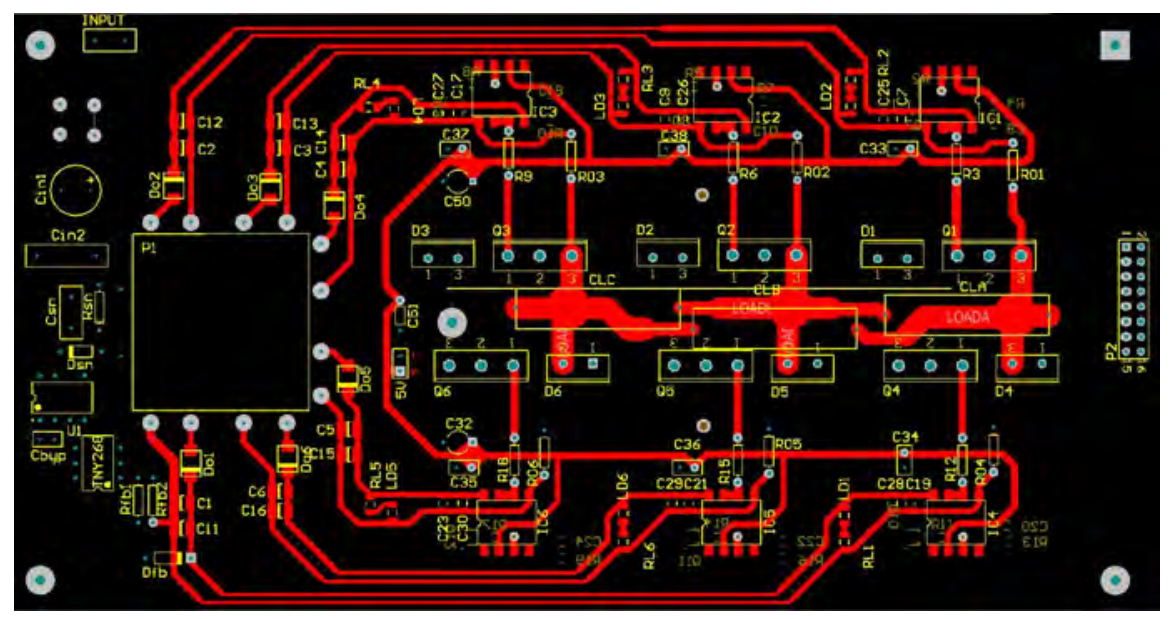

(b) Cara superior

Figura A.6: circuito impreso de un módulo.

\section{A.4. Carga trifásica variable}

La salida del convertidor se aplica a una carga $R L$ en estrella, con posibilidad de variar sus parámetros para poder verificar que es posible alimentar cargas inductivas, generar potencias activa y reactiva en forma independiente y evaluar la respuesta dinámica del convertidor. Para ello se utiliza un contactor trifásico operado en forma remota desde la FPGA. El contactor conecta la mitad de la parte resistiva de cada fase con el neutro, produciendo una variación entre 16 y $8 \Omega$. Se utiliza esta configuración a fin de vitar que durante las conmutaciones la carga pueda quedar momentáneamente desconectada debido al tiempo de accionamiento de los contactos mecánicos del contactor, lo cual podría producir sobretensiones en el convertidor fuente de corriente. 


\section{A.5. Prototipo}

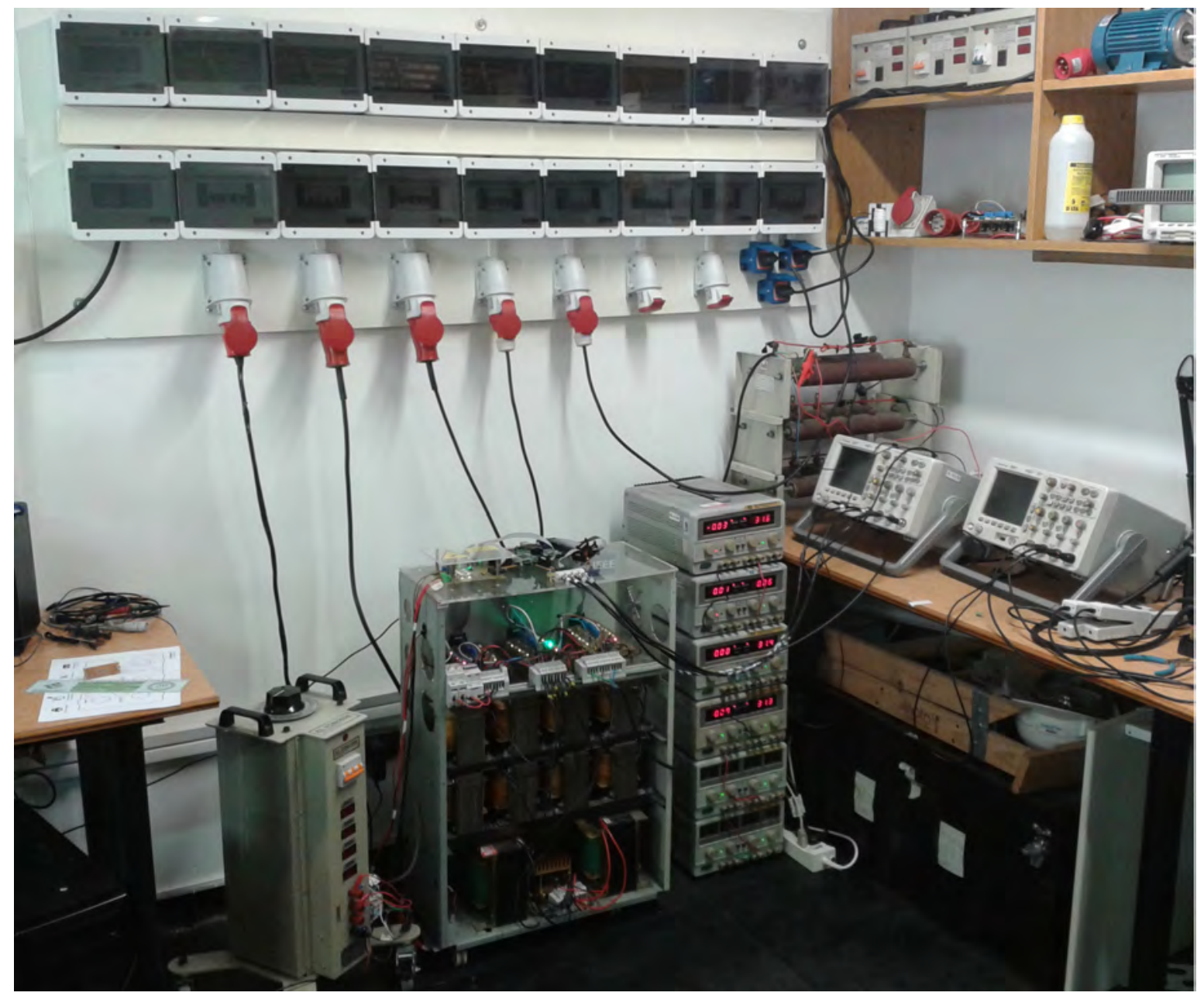

Figura A.7: vista general del arreglo de laboratorio para conexionado y pruebas. 


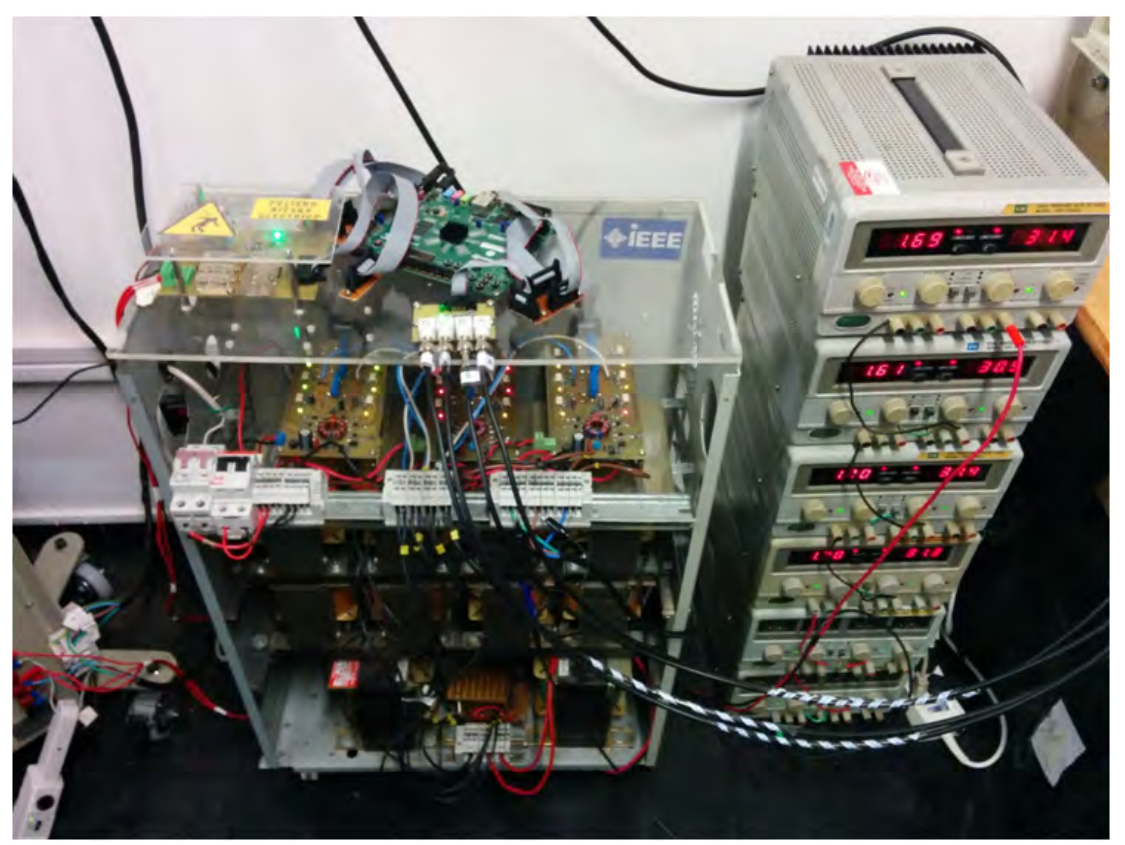

Figura A.8: detalle del prototipo y la fuente de CC de laboratorio.

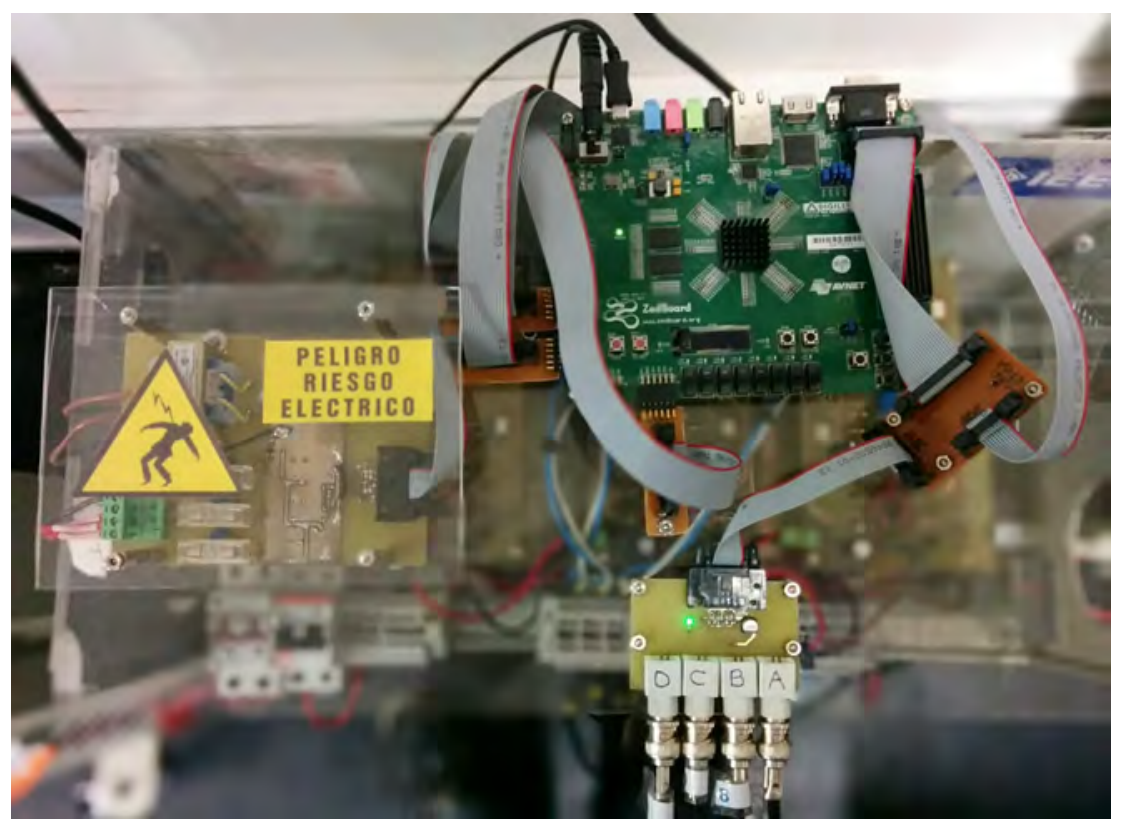

Figura A.9: sistema de adquisición de datos y FPGA de control. 


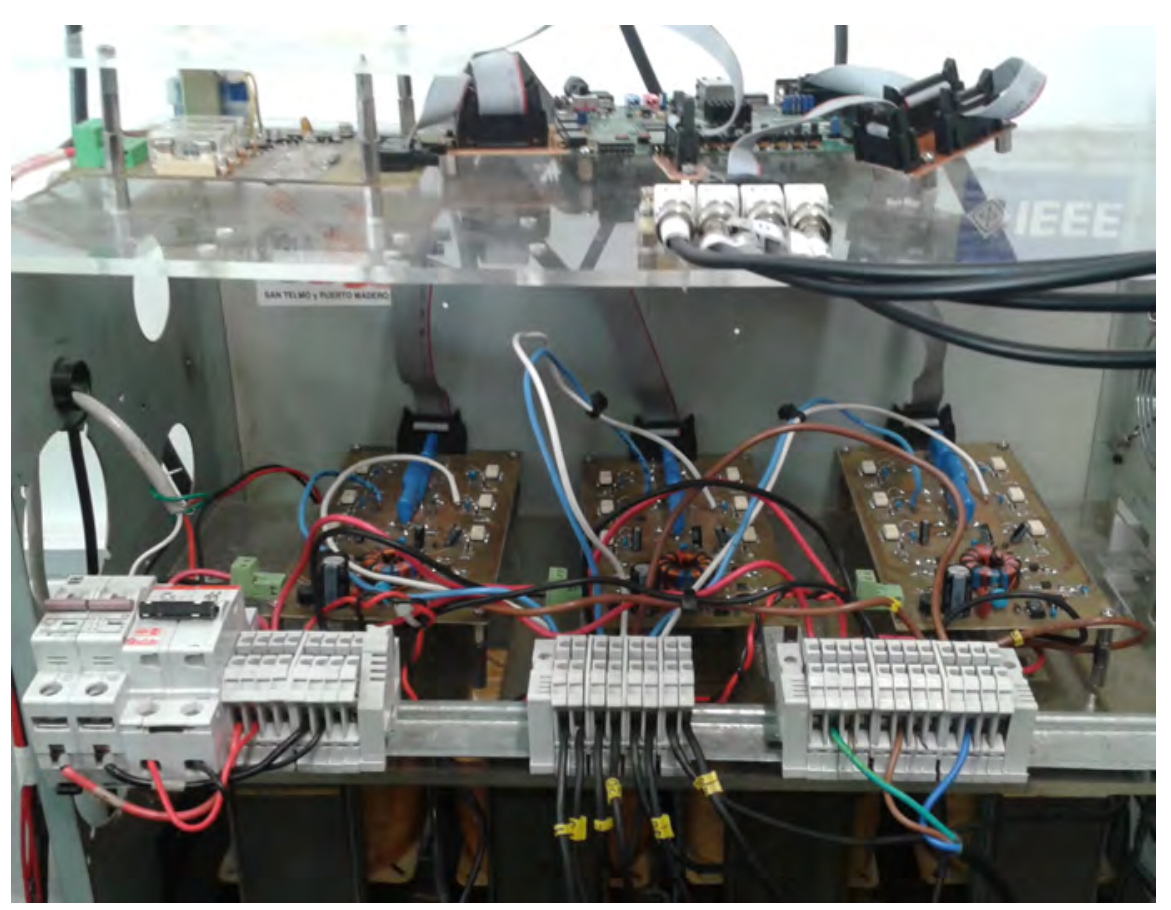

Figura A.10: detalle del conexionado de los módulos.

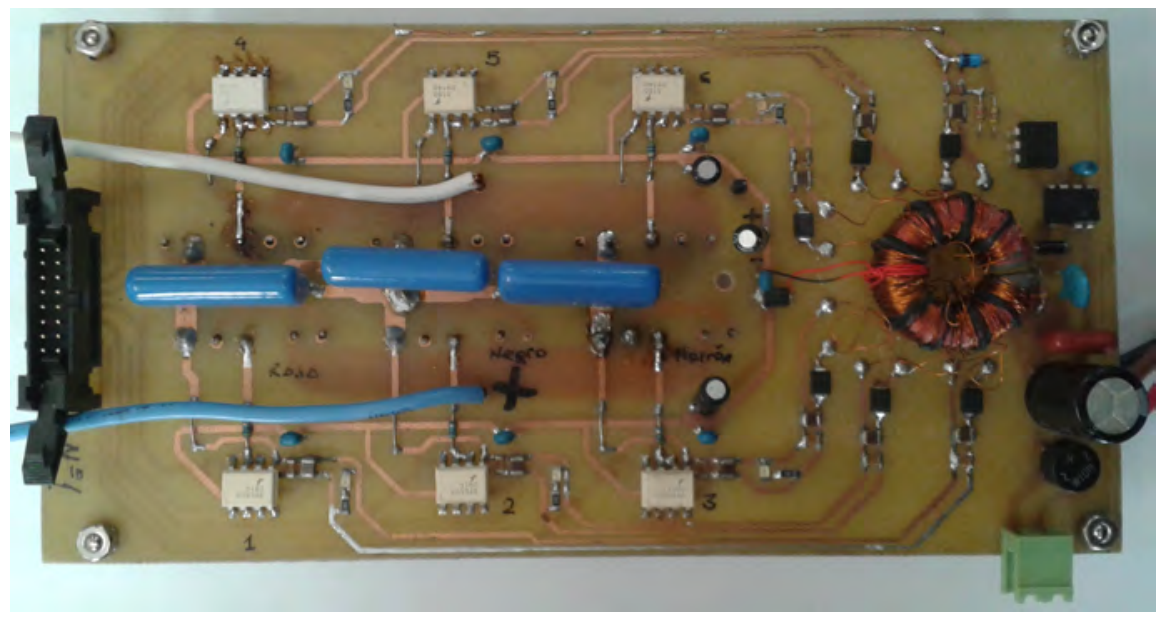

(a) Vista superior.

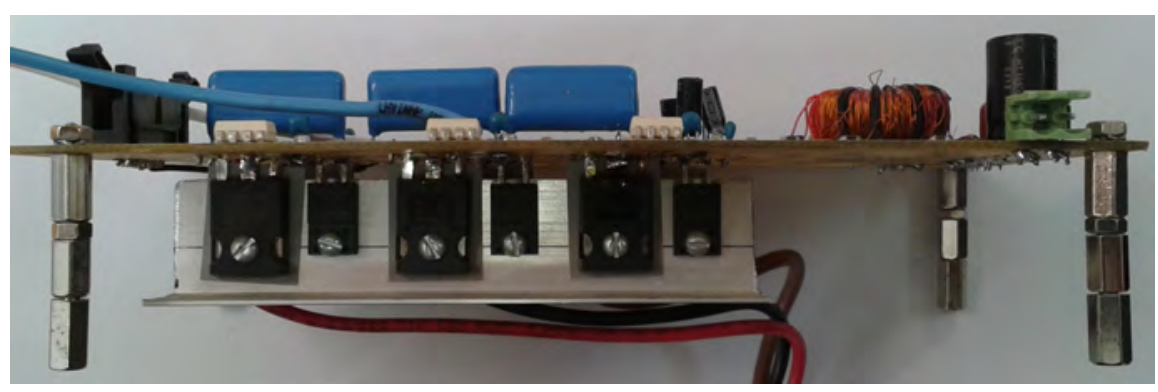

(b) Vista lateral.

Figura A.11: detalle de un módulo. 


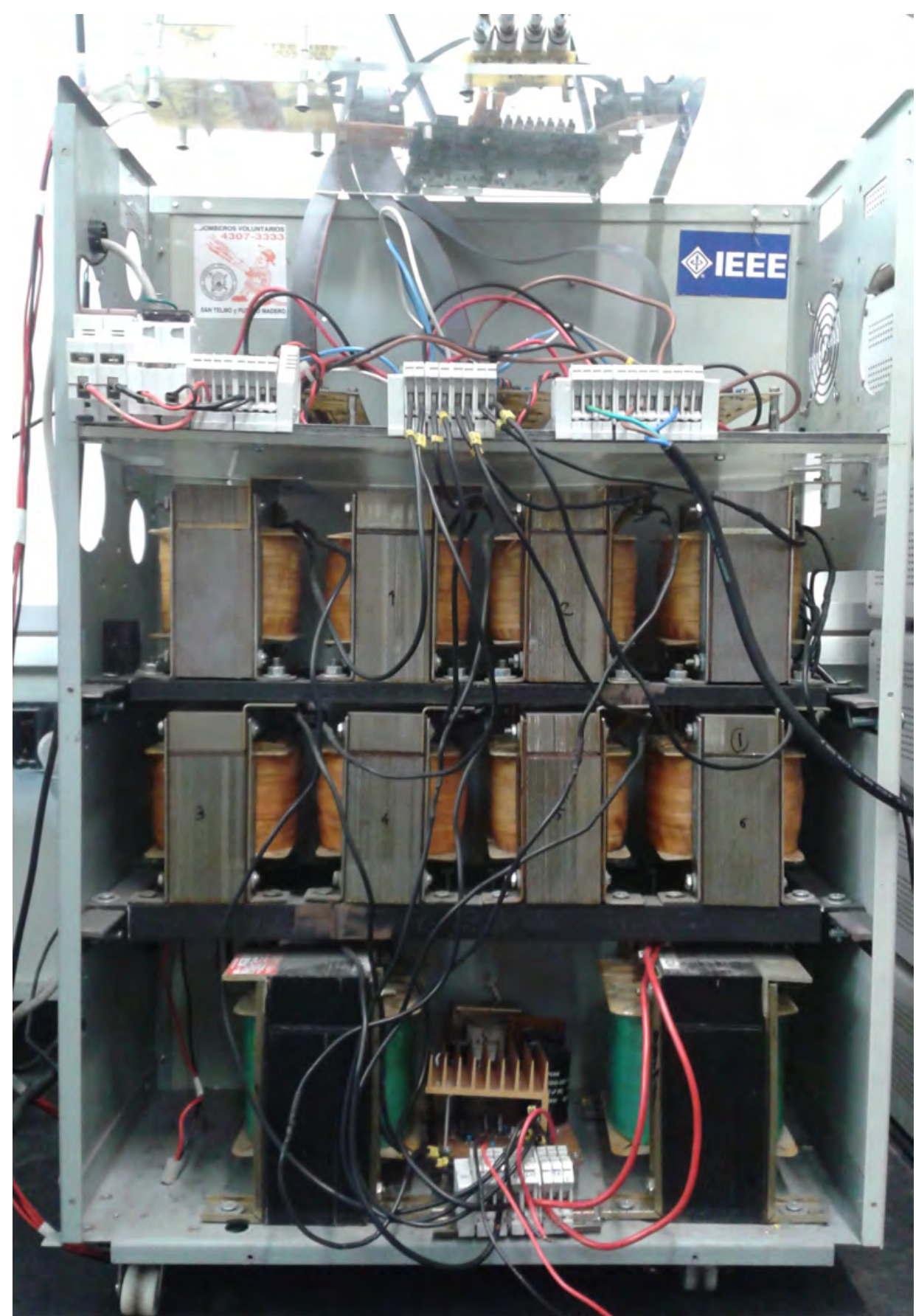

Figura A.12: vista de los inductores divisores (arriba) e inductores principales (abajo). 


\section{Bibliografía}

Adam, G., K. Ahmed, S. Finney, K. Bell, y B. Williams. New breed of network fault-tolerant voltage-source-converter hvdc transmission system. Power Systems, IEEE Transactions on, 28(1):335-346 (2013). ISSN 0885-8950.

Aguirre, M., M. Angélico, J. Bracco, y M. Valla. Balance de corrientes en un convertidor multinivel fuente de corriente. En AADECA2012, XXIII Congreso Argentino de Control Automático (2012a).

Aguirre, M., L. Calvino, V. Corasaniti, y M. Valla. Multilevel current source inverter to improve power quality in a distribution network. En Industrial Electronics (ISIE), 2010 IEEE International Symposium on, págs. 3292 -3297 (2010a).

Aguirre, M., L. Calvino, L. Slobozian, y M. Valla. Protección contra fallas en convertidores de modo corriente. En RPIC2009, XIII Reunión de Trabajo en Procesamiento de la Información y Control (2009).

Aguirre, M., L. Calvino, y M. Valla. Multilevel current-source inverter with fpga control. Industrial Electronics, IEEE Transactions on, 60(1):3 -10 (2013a). ISSN 0278-0046.

Aguirre, M., L. Calviño, y M. Valla. Fault tolerant multilevel current source inverter. En Industrial Technology (ICIT), 2010 IEEE International Conference on, págs. 1345 -1350 (2010b).

Aguirre, M., P. Cossutta, J. Bracco, y M. Valla. Sistema de acoplamiento a la red para microgeneración fotovoltaica distribuida. En HYFUSEN2013, V Congreso Nacional, IV Congreso Iberoamericano Hidrógeno y Fuentes Sustentables de Energía (2013b).

Aguirre, M., H. Couto, y M. I. Valla. Analysis and simulation of a hydrogen based electric system to improve power quality in distributed grids. International Journal of Hydrogen Energy, 37(19):14959-14965 (2012b).

Aguirre, M., M. Engelhardt, J. Bracco, y M. Valla. Current balance control in a multilevel current source inverter. En Industrial Technology (ICIT), 2013 IEEE International Conference on, págs. 1567-1572 (2013c). 
Aguirre, M., C. L., F. Corasaniti, y M. Valla. Filtro activo de modo corriente multinivel para mejorar la calidad de potencia en sistemas de distribución. En AADECA2010, XXII Congreso Argentino de Control Automático, tomo AF003328 (2010c).

Aguirre, M., A. Latini, H. Couto, y M. Valla. Análisis y simulación de un sistema initerrumpido de energia mediante almacenamiento de hidrógeno. En HYFUSEN2011, IV Congreso Nacional, III Congreso Iberoamericano Hidrógeno y Fuentes Sustentables de Energía, tomo 02 (2011a).

Aguirre, M., A. Latini, H. Couto, y M. Valla. Convertidor bidireccional para la produccion de hidrogeno y generacion de electricidad. En RPIC2011, XIV Reunión de Trabajo en Procesamiento de la Información y Control (2011b).

Aguirre, M., A. Latini, H. Couto, y M. Valla. Sincronización con la red de un convertidor multinivel fuente de corriente con control de balance para generación fotovoltaica. En RPIC2013, XV Reunión de Trabajo en Procesamiento de la Información y Control (2013d).

Aguirre, M. y M. Valla. An environmental friendly alternative for hydrogen production and electric energy generation. En IECON 2011 - 37th Annual Conference on IEEE Industrial Electronics Society, págs. 3099 -3104 (2011). ISSN 1553-572X.

Akagi, H., E. H. Watanabe, y M. Aredes. Instantaneous Power Theory and Applications to Power Conditioning. IEEE Press, 445 Hoes Lane, Piscataway, NJ 08854 (2007). ISBN 978-0-470-10761-4.

Al-Hanai, T., T. Al-Hanaei, S. Veeranna, y A. Beig. Effect of gate pulse variation on the performance of fifteen-level cascaded h-bridge voltage source inverter. En GCC Conference and Exhibition (GCC), 2011 IEEE, págs. 85-88 (2011).

Alepuz, S., S. Busquets-Monge, J. Bordonau, J. Gago, D. Gonzalez, y J. Balcells. Interfacing renewable energy sources to the utility grid using a three-level inverter. Industrial Electronics, IEEE Transactions on, 53(5):1504-1511 (2006). ISSN 0278-0046.

Antunes, F. L. M., H. A. C. Braga, y I. Barbi. Application of a generalized current multilevel cell to current-source inverters. Industrial Electronics, IEEE Transactions on, 46(1):31-38 (1999). ISSN 0278-0046.

Bai, Z. y Z. Zhang. Conformation of multilevel current source converter topologies using the duality principle. Power Electronics, IEEE Transactions on, 23(5):2260 -2267 (2008a). ISSN 0885-8993.

Bai, Z. y Z. Zhang. Digital control technique for multi-module current source converter. En Industrial Technology, 2008. ICIT 2008. IEEE International Conference on, págs. $1-5$ (2008b).

Bai, Z., Z. Zhang, y G. Chen. Development of a three-phase high power factor multilevel current-source rectifier. En Power Electronics Specialists Conference, 2008. PESC 2008. IEEE, págs. $574-578$ (2008). ISSN 0275-9306. 
Bai, Z., Z. Zhang, y X. Ruan. A natural soft-commutation pwm scheme for current source converter and its logic implementation. Industrial Electronics, IEEE Transactions on, 58(7):2772 -2779 (2011). ISSN 0278-0046.

Bai, Z., Z. Zhang, y Y. Zhang. A generalized three-phase multilevel current source inverter with carrier phase-shifted spwm. En Power Electronics Specialists Conference, 200\%. PESC 200\%. IEEE, págs. 2055-2060 (2007). ISSN 0275-9306.

Barros, J. y J. Silva. Optimal predictive control of three-phase npc multilevel converter for power quality applications. Industrial Electronics, IEEE Transactions on, 55(10):36703681 (2008). ISSN 0278-0046.

Beig, A. y V. Ranganathan. A novel csi-fed induction motor drive. Power Electronics, IEEE Transactions on, 21(4):1073-1082 (2006). ISSN 0885-8993.

Bhattacharya, A., C. Chakraborty, y S. Bhattacharya. Shunt compensation. Industrial Electronics Magazine, IEEE, 3(3):38-49 (2009). ISSN 1932-4529.

Blahnik, V., Z. Peroutka, J. Zak, y J. Talla. Elimination of dead-time effect causing current distortion of single-phase power converters. En Power Electronics and Motion Control Conference (EPE/PEMC), 2012 15th International, págs. DS1e.3-1-DS1e.3-6 (2012).

Ceballos, S., J. Pou, S. Choi, M. Saeedifard, y V. Agelidis. Analysis of voltage balancing limits in modular multilevel converters. En IECON 2011 - 37th Annual Conference on IEEE Industrial Electronics Society, págs. 4397 -4402 (2011). ISSN 1553-572X.

Ceballos, S., J. Pou, I. Gabiola, J. Villate, J. Zaragoza, y D. Boroyevich. Fault-tolerant multilevel converter topology. En Industrial Electronics, 2006 IEEE International Symposium on, tomo 2, págs. 1577-1582 (2006).

Ceballos, S., J. Pou, E. Robles, J. Zaragoza, P. Ibanez, y J. Martin. Fault-tolerant hybrid fourleg multilevel converter. En Power Electronics and Applications, 2007 European Conference on, págs. 1-9 (2007).

Chatzakis, J. y E. Antonidakis. A novel $\mathrm{n}+\mathrm{k}$ fault-tolerant hot-swap dc/ac inverter design. En Power Electronics Specialists Conference, 2008. PESC 2008. IEEE, págs. 3291-3294 (2008). ISSN 0275-9306.

Chen, A. y X. He. Research on hybrid-clamped multilevel-inverter topologies. Industrial Electronics, IEEE Transactions on, 53(6):1898 -1907 (2006). ISSN 0278-0046.

Chen, A., L. Hu, L. Chen, Y. Deng, G. Yao, y X. He. A multilevel converter topology with fault tolerant ability. En Applied Power Electronics Conference and Exposition, 2004. APEC '04. Nineteenth Annual IEEE, tomo 3, págs. 1610-1616 Vol.3 (2004).

Chen, A., C. Zhang, H. Ma, y Y. Deng. A novel multilevel inverter topology with no clamping diodes and flying capacitors. En Industrial Electronics, 2008. IECON 2008. 34th Annual Conference of IEEE, págs. 3184-3187 (2008). ISSN 1553-572X. 
Ciobotaru, M., F. Iov, P. Zanchetta, Y. De Novaes, y F. Blaabjerg. Study and analysis of a natural reference frame current controller for a multi-level h-bridge power converter. En Power Electronics Specialists Conference, 2008. PESC 2008. IEEE, págs. 2914-2920 (2008). ISSN 0275-9306.

Corasaniti, F., M. Barbieri, P. Arnera, y M. Valla. Load characterization in medium voltage of an electric distribution utility related to active filters. En Transmission Distribution Conference and Exposition: Latin America, 2006. TDC '06. IEEE/PES, págs. 1 -7 (2006).

Corasaniti, V., M. Barbieri, P. Arnera, y M. Valla. Reactive and harmonics compensation in a medium voltage distribution network with active filters. En Industrial Electronics, $200 \%$. ISIE 200\%. IEEE International Symposium on, págs. 2510-2515 (2007).

Corasaniti, V., M. Barbieri, P. Arnera, y M. Valla. Hybrid power filter to enhance power quality in a medium-voltage distribution network. Industrial Electronics, IEEE Transactions on, 56(8):2885 -2893 (2009). ISSN 0278-0046.

Corasaniti, V., M. B. Barbieri, P. Arnera, y M. Valla. Comparison of active filters topologies in medium voltage distribution power systems. En Power and Energy Society General Meeting - Conversion and Delivery of Electrical Energy in the 21st Century, 2008 IEEE, págs. 1-8 (2008). ISSN 1932-5517.

Cordeiro, A., J. Silva, S. Pinto, y J. Santana. Fault-tolerant design for a three-level neutralpoint-clamped multilevel inverter topology. En EUROCON - International Conference on Computer as a Tool (EUROCON), 2011 IEEE, págs. 1 -4 (2011).

Correa, P., M. Pacas, y J. Rodriquez. Modulation strategies for fault-tolerant operation of hbridge multilevel inverters. En Industrial Electronics, 2006 IEEE International Symposium on, tomo 2, págs. 1589 -1594 (2006).

de Alvarenga, M. y J. Pomilio. Analysis of pulse suppression in pwm modulation for symmetric cascaded multilevel inverters. En IECON 2011 - 37th Annual Conference on IEEE Industrial Electronics Society, págs. 4654-4659 (2011). ISSN 1553-572X.

Dijkhuizen, F. y S. Norrga. Fault tolerant operation of power converter with cascaded cells. En Power Electronics and Applications (EPE 2011), Proceedings of the 2011-14th European Conference on, págs. 1-8 (2011).

Dixon, J., P. Barriuso, M. Ortuzar, L. Moran, J. Pontt, y J. Rodriguez. Fault tolerant reconfiguration system for asymmetric multilevel converters using bi-directional power switches. En Industrial Electronics Society, 200\%. IECON 200\%. 33rd Annual Conference of the IEEE, págs. $2124-2129$ (2007). ISSN 1553-572X.

Dixon, J. y L. Moran. Multilevel inverter, based on multi-stage connection of three-level converters scaled in power of three. En IECON 02 [Industrial Electronics Society, IEEE 2002 28th Annual Conference of the], tomo 2, págs. 886 - 891 vol.2 (2002). 
Dupczak, B., A. Perin, y M. Heldwein. Space vector modulation strategy applied to interphase transformers-based five-level current source inverters. Power Electronics, IEEE Transactions on, 27(6):2740-2751 (2012). ISSN 0885-8993.

Ellis, M., M. Von Spakovsky, y D. Nelson. Fuel cell systems: efficient, flexible energy conversion for the 21st century. Proceedings of the IEEE, 89(12):1808-1818 (2001). ISSN 0018-9219.

Erickson, R. y D. Maksimovic. Fundamentals of Power Electronics, 2nd. Edition. Springer (2012).

Ertl, H., J. Kolar, y F. C. Zach. A novel multicell dc-ac converter for applications in renewable energy systems. Industrial Electronics, IEEE Transactions on, 49(5):1048-1057 (2002). ISSN 0278-0046.

Espinoza, J. y G. Joos. On-line generation of gating signals for current source converter topologies. En Industrial Electronics, 1993. Conference Proceedings, ISIE'93 - Budapest., IEEE International Symposium on, págs. 674 -678 (1993).

Ewanchuk, J., J. Salmon, y B. Vafakhah. A five-/nine-level twelve-switch neutral-pointclamped inverter for high-speed electric drives. Industry Applications, IEEE Transactions on, 47(5):2145 -2153 (2011). ISSN 0093-9994.

Flores, P., J. Dixon, M. Ortuzar, R. Carmi, P. Barriuso, y L. Moran. Static var compensator and active power filter with power injection capability, using 27-level inverters and photovoltaic cells. Industrial Electronics, IEEE Transactions on, 56(1):130-138 (2009). ISSN 0278-0046.

Franquelo, L., J. Rodriguez, J. Leon, S. Kouro, R. Portillo, y M. Prats. The age of multilevel converters arrives. Industrial Electronics Magazine, IEEE, 2(2):28 -39 (2008). ISSN 19324529 .

Friede, W., S. Rael, y B. Davat. Mathematical model and characterization of the transient behavior of a pem fuel cell. Power Electronics, IEEE Transactions on, 19(5):1234 - 1241 (2004). ISSN 0885-8993.

Gao, F., P. C. Loh, F. Blaabjerg, y D. Vilathgamuwa. Five-level current-source inverters with buck-boost and inductive-current balancing capabilities. Industrial Electronics, IEEE Transactions on, 57(8):2613-2622 (2010). ISSN 0278-0046.

Gonzalez, S., M. Valla, y C. Christiansen. Five-level cascade asymmetric multilevel converter. Power Electronics, IET, 3(1):120 -128 (2010). ISSN 1755-4535.

Grandi, G., Y. Gritli, F. Filippetti, y C. Rossi. Fault-tolerant operating analysis of a quadinverter multiphase multilevel ac motor drive. En Diagnostics for Electric Machines, Power Electronics Drives (SDEMPED), 2011 IEEE International Symposium on, págs. 126 -132 (2011). 
Grandi, G., C. Rossi, D. Ostojic, y D. Casadei. A new multilevel conversion structure for gridconnected pv applications. Industrial Electronics, IEEE Transactions on, 56(11):4416-4426 (2009). ISSN 0278-0046.

Hingorani, N. G. y L. Gyugyi. Understanding FACTS, Concepts and technology of Flexible AC Transmission Systems. IEEE Press, 445 Hoes Lane, P.O. Box 1331, Piscataway, NJ 08855-1331 (2000).

Hoffmann, N., R. Lohde, M. Fischer, F. Fuchs, L. Asiminoaei, y P. Thogersen. A review on fundamental grid-voltage detection methods under highly distorted conditions in distributed power-generation networks. En Energy Conversion Congress and Exposition (ECCE), 2011 IEEE, págs. 3045-3052 (2011).

Holmes, D. G. y T. A. Lipo. Pulse width modulation for power convertes. Principles and practice. IEEE Press, 445 Hoes Lane, Piscataway, NJ 08854 (2003).

Holtz, J. Pulsewidth modulation-a survey. Industrial Electronics, IEEE Transactions on, 39(5):410-420 (1992). ISSN 0278-0046.

Hombu, M., S. Ueda, A. Ueda, y Y. Matsuda. A new current source gto inverter with sinusoidal output voltage and current. Industry Applications, IEEE Transactions on, IA21(5):1192-1198 (1985). ISSN 0093-9994.

Hu, P., D. Jiang, Y. Zhou, Y. Liang, J. Guo, y Z. Lin. Energy balancing control strategy for modular multilevel converters under sub-module fault conditions. Power Electronics, IEEE Transactions on, $\mathrm{PP}(99): 1-1$ (2013). ISSN 0885-8993.

IEEE. IEEE Std 519 1992. IEEE Recommended Practices and Requirements for Harmonic Control in Electrical Power Systems (1993).

Ishikawa, H. y Y. Murai. A novel soft-switched pwm current source inverter with voltage clamped circuit. Power Electronics, IEEE Transactions on, 15(6):1081-1087 (2000). ISSN 0885-8993.

Jalakas, T., D. Vinnikov, y J. Laugis. Evaluation of different loss calculation methods for high-voltage igbt-s under small load conditions. págs. 1263 -1267 (2008).

Jana, K., S. Biswas, y P. Thakura. A simple and generalized space vector pwm control of cascaded h-bridge multilevel inverters. En Industrial Technology, 2006. ICIT 2006. IEEE International Conference on, págs. 1281-1286 (2006).

Kim, H. G., S. Sul, y M. H. Park. Optimal efficiency drive of a current source inverter fed induction motor by flux control. Industry Applications, IEEE Transactions on, IA20(6):1453-1459 (1984). ISSN 0093-9994.

Kou, X., K. Corzine, y Y. Familiant. A unique fault-tolerant design for flying capacitor multilevel inverter. Power Electronics, IEEE Transactions on, 19(4):979 - 987 (2004). ISSN 0885-8993. 
Krug, D., S. Bernet, S. Fazel, K. Jalili, y M. Malinowski. Comparison of 2.3-kv mediumvoltage multilevel converters for industrial medium-voltage drives. Industrial Electronics, IEEE Transactions on, 54(6):2979 -2992 (2007). ISSN 0278-0046.

Kunusch, C., P. F. Puleston, M. A. Mayosky, y J. J. Moré. Characterization and experimental results in pem fuel cell electrical behaviour. International Journal of Hydrogen Energy, 35(11):5876-5881 (2010).

Kwak, S. y H. Toliyat. Multilevel converter topology using two types of current-source inverters. Industry Applications, IEEE Transactions on, 42(6):1558-1564 (2006). ISSN 0093-9994.

Lai, J.-S. y F. Z. Peng. Multilevel converters-a new breed of power converters. Industry Applications, IEEE Transactions on, 32(3):509 -517 (1996). ISSN 0093-9994.

Lakshminarayanan, S., G. Mondal, P. N. Tekwani, K. Mohapatra, y K. Gopakumar. Twelvesided polygonal voltage space vector based multilevel inverter for an induction motor drive with common-mode voltage elimination. Industrial Electronics, IEEE Transactions on, 54(5):2761-2768 (2007). ISSN 0278-0046.

Lauretta, J. Producción y almacenamiento de hidrógeno a alta presión. 1er.Congreso Nacional Hidrógeno y Energías Sustentables, (8-35) (2005).

Li, J., A. Huang, S. Bhattacharya, y S. Lukic. Eto light multilevel converters for large electric vehicle and hybrid electric vehicle drives. En Vehicle Power and Propulsion Conference, 2009. VPPC '09. IEEE, págs. 1455-1460 (2009).

Li, S. y L. Xu. Strategies of fault tolerant operation for three-level pwm inverters. Power Electronics, IEEE Transactions on, 21(4):933 - 940 (2006). ISSN 0885-8993.

Liang, J., A. Nami, F. Dijkhuizen, P. Tenca, y J. Sastry. Current source modular multilevel converter for hvdc and facts. En Power Electronics and Applications (EPE), 2013 15th European Conference on, págs. 1-10 (2013).

Lu, Z.-G., L.-L. Zhao, W.-P. Zhu, C.-J. Wu, y Y.-S. Qin. Research on cascaded three-phasebridge multilevel converter based on cps-pwm. Power Electronics, IET, 6(6):1088-1099 (2013). ISSN 1755-4535.

Luo, F. L. y H. Ye. Advanced Multi-Quadrant Operation DC/DC Converters. CRC Press (2005). ISBN 978-0-849-37239-1.

Ma, M., L. Hu, A. Chen, y X. He. Reconfiguration of carrier-based modulation strategy for fault tolerant multilevel inverters. Power Electronics, IEEE Transactions on, 22(5):2050 -2060 (2007). ISSN 0885-8993.

Malinowski, M., K. Gopakumar, J. Rodriguez, y M. Perez. A survey on cascaded multilevel inverters. Industrial Electronics, IEEE Transactions on, 57(7):2197-2206 (2010). ISSN 0278-0046. 
Marchesoni, M., M. Mazzucchelli, y P. Tenca. About the dc-link capacitors voltage balance in multi-point clamped converters. En Industrial Electronics Society, 1998. IECON '98. Proceedings of the 24th Annual Conference of the IEEE, tomo 1, págs. 548 -553 vol.1 (1998).

Marchesoni, M. y P. Tenca. Diode-clamped multilevel converters: a practicable way to balance dc-link voltages. Industrial Electronics, IEEE Transactions on, 49(4):752 - 765 (2002). ISSN 0278-0046.

McGrath, B. y D. Holmes. Natural current balancing of multicell current source converters. Power Electronics, IEEE Transactions on, 23(3):1239 -1246 (2008a). ISSN 0885-8993.

McGrath, B. P. y D. G. Holmes. Analytical modelling of voltage balance dynamics for a flying capacitor multilevel converter. IEEE Trans. Power Electron., 49(2):543-550 (2008b).

Mendes, A., X. Lopez-Fernandez, y A. Marques Cardoso. Thermal performance of a threephase induction motor under fault tolerant operating strategies. Power Electronics, IEEE Transactions on, 23(3):1537 -1544 (2008). ISSN 0885-8993.

Mohan, N., T. M. Undeland, y W. P. Robbins. Power Electronics: Converters, Applications, and Design. 3rd. Edition.. John Wiley \& Sons (2005). ISBN 978-0-471-22693-2.

Murray, N., J. Arrillaga, N. Watson, y Y. Liu. Four quadrant multilevel current source power conditioning for superconductive magnetic energy storage. En Power Engineering Conference, 2009. AUPEC 2009. Australasian Universities, págs. 1-5 (2009).

Nabae, A., I. Takahashi, y H. Akagi. A new neutral-point-clamped pwm inverter. Industry Applications, IEEE Transactions on, IA-17(5):518 -523 (1981). ISSN 0093-9994.

Nonaka, S. y Y. Neba. Analysis of pwm gto current source inverter-fed induction motor drive system. Industry Applications, IEEE Transactions on, IA-23(2):256-262 (1987). ISSN 0093-9994.

Park, J. K., K. M. Cho, W. S. Oh, K. Sol, y C. G. In. Analysis of the errors on the output voltage of inverter caused by switching dead times. En Power Electronics, Electrical Drives, Automation and Motion, 2008. SPEEDAM 2008. International Symposium on, págs. 987991 (2008).

Peng, F., W. Qian, y D. Cao. Recent advances in multilevel converter/inverter topologies and applications. En Power Electronics Conference (IPEC), 2010 International, págs. 492-501 (2010).

Peng, F. Z. A generalized multilevel inverter topology with self voltage balancing. Industry Applications, IEEE Transactions on, 37(2):611 -618 (2001). ISSN 0093-9994.

Powerint. Tny263-268 tinyswitch-ii family. 5245 Hellyer Avenue, San Jose, CA 95138 USA (2009). 
Rech, C. y J. Pinheiro. Hybrid multilevel converters: Unified analysis and design considerations. Industrial Electronics, IEEE Transactions on, 54(2):1092 -1104 (2007). ISSN 0278-0046.

Rodriguez, J., S. Bernet, P. Steimer, y I. Lizama. A survey on neutral-point-clamped inverters. Industrial Electronics, IEEE Transactions on, 57(7):2219-2230 (2010). ISSN 0278-0046.

Rodriguez, J., S. Bernet, B. Wu, J. Pontt, y S. Kouro. Multilevel voltage-source-converter topologies for industrial medium-voltage drives. Industrial Electronics, IEEE Transactions on, 54(6):2930 -2945 (2007). ISSN 0278-0046.

Rodriguez, J., L. Franquelo, S. Kouro, J. Leon, R. Portillo, M. Prats, y M. Perez. Multilevel converters: An enabling technology for high-power applications. tomo 97, págs. 1786-1817 (2009). ISSN 0018-9219.

Rodriguez, J., J.-S. Lai, y F. Z. Peng. Multilevel inverters: a survey of topologies, controls, and applications. Industrial Electronics, IEEE Transactions on, 49(4):724-738 (2002). ISSN 0278-0046.

Rodriguez, J., J. Pontt, E. Silva, J. Espinoza, y M. Perez. Topologies for regenerative cascaded multilevel inverters. En Power Electronics Specialist Conference, 2003. PESC '03. 2003 IEEE 34th Annual, tomo 2, págs. 519-524 vol.2 (2003). ISSN 0275-9306.

Samuel, P., R. Gupta, y D. Chandra. Grid interface of wind power with large split-winding alternator using cascaded multilevel inverter. Energy Conversion, IEEE Transactions on, 26(1):299-309 (2011). ISSN 0885-8969.

Shen, K., B. Xiao, J. Mei, L. Tolbert, J. Wang, X. Cai, y Y. Ji. A modulation reconfiguration based fault-tolerant control scheme for modular multilevel converters. En Applied Power Electronics Conference and Exposition (APEC), 2013 Twenty-Eighth Annual IEEE, págs. 3251-3255 (2013). ISSN 1048-2334.

Sirisukprasert, S. y T. Saengsuwan. The modeling and control of fuel cell emulators. En Electrical Engineering/Electronics, Computer, Telecommunications and Information Technology, 2008. ECTI-CON 2008. 5th International Conference on, tomo 2, págs. 985 -988 (2008).

Smoglie, C. y J. Lauretta. An efficiency model for hydrogen production in a pressurized electrolyzer. XXI World Energy Congress (2010).

Son, G. T., H.-J. Lee, T. S. Nam, Y.-H. Chung, U.-H. Lee, S.-T. Baek, K. Hur, y J.-W. Park. Design and control of a modular multilevel hvdc converter with redundant power modules for noninterruptible energy transfer. Power Delivery, IEEE Transactions on, 27(3):16111619 (2012). ISSN 0885-8977.

Souleman, N., O. Tremblay, y L.-A. Dessaint. A generic fuel cell model for the simulation of fuel cell power systems. En Power Energy Society General Meeting, 2009. PES '09. IEEE, págs. 1 -8 (2009). ISSN 1944-9925. 
Suh, Y., J. Steinke, y P. Steimer. Efficiency comparison of voltage-source and current-source drive systems for medium-voltage applications. Industrial Electronics, IEEE Transactions on, 54(5):2521-2531 (2007). ISSN 0278-0046.

Suresh, Y. y A. Panda. Research on a cascaded multilevel inverter by employing three-phase transformers. Power Electronics, IET , 5(5):561-570 (2012). ISSN 1755-4535.

Suroso y T. Noguchi. Common-emitter topology of multilevel current-source pulse width modulation inverter with chopper-based dc current sources. Power Electronics, IET, 4(7):759-766 (2011). ISSN 1755-4535.

Suroso, S. y T. Noguchi. New h-bridge multilevel current-source pwm inverter with reduced switching device count. En Power Electronics Conference (IPEC), 2010 International, págs. 1228-1235 (2010).

Suroso, S. y T. Noguchi. Multilevel current waveform generation using inductor cells and hbridge current-source inverter. Power Electronics, IEEE Transactions on, 27(3):1090-1098 (2012). ISSN 0885-8993.

Terciyanli, A., T. Avci, I. Yilmaz, C. Ermis, K. Kose, A. Acik, A. Kalaycioglu, Y. Akkaya, I. Cadirci, y M. Ermis. A current source converter-based active power filter for mitigation of harmonics at the interface of distribution and transmission systems. Industry Applications, IEEE Transactions on, 48(4):1374-1386 (2012). ISSN 0093-9994.

Tettamanti, J., A. Latini, y M. Aguirre. An example of rapid design of power electronics control with fpga in matlab/simulink. En SPL2011, VII Southern Conference on Programmable Logic, págs. 69 -74 (2011). ISBN 978-1-4244-8846-9.

Valla, M. I., S. A. González, y S. A. Verne. Multilevel Converters for Industrial Applications. CRC Press, 6000 Broken Sound Parkway NW, Suite 300, Boca Raton, FL 33487-2742 (2013).

Vazquez, N., H. Lopez, C. Hernandez, E. Vazquez, R. Osorio, y J. Arau. A different multilevel current-source inverter. Industrial Electronics, IEEE Transactions on, 57(8):2623-2632 (2010). ISSN 0278-0046.

Verma, A. K., P. Thakura, K. Jana, y G. Buja. Cascaded multilevel inverter for hybrid electric vehicles. En Power Electronics (IICPE), 2010 India International Conference on, págs. 1-6 (2011).

Verne, S. y M. Valla. Predictive control of a back to back diode clamped multilevel converter. En Industrial Technology (ICIT), 2010 IEEE International Conference on, págs. 1814 1819 (2010a).

Verne, S. y M. Valla. Predictive control of a back to back motor drive based on diode clamped multilevel converters. En IECON 2010 - 36th Annual Conference on IEEE Industrial Electronics Society, págs. 2972-2977 (2010b). ISSN 1553-572X. 
Wakerly, J. F. Digital Design Principles and Practices. Pearson Education, Inc., Upper Saddle River, NJ (2006). ISBN 0-13-186389-4.

Wang, C., M. Nehrir, y H. Gao. Control of pem fuel cell distributed generation systems. Energy Conversion, IEEE Transactions on, 21(2):586 - 595 (2006). ISSN 0885-8969.

Wang, X. y B.-T. Ooi. Unity pf current-source rectifier based on dynamic trilogic pwm. Power Electronics, IEEE Transactions on, 8(3):288-294 (1993). ISSN 0885-8993.

Welchko, B., T. Lipo, T. Jahns, y S. Schulz. Fault tolerant three-phase ac motor drive topologies: a comparison of features, cost, and limitations. Power Electronics, IEEE Transactions on, 19(4):1108 - 1116 (2004). ISSN 0885-8993.

Xin, K. y A. Khambadkone. Dynamic modelling of fuel cell with power electronic current and performance analysis. En Power Electronics and Drive Systems, 2003. PEDS 2003. The Fifth International Conference on, tomo 1, págs. 607 - 612 (2003).

Xiong, Y., D. Chen, X. Yang, C. Hu, y Z. Zhang. Analysis and experimentation of a new threephase multilevel current-source inverter. En Power Electronics Specialists Conference, 2004. PESC 04. 2004 IEEE 35th Annual, tomo 1, págs. 548 - 551 Vol.1 (2004a). ISSN 0275-9306.

Xiong, Y., D. Chen, X. Yang, C. Hu, y Z. Zhang. Analysis and experimentation of a new threephase multilevel current-source inverter. En Power Electronics Specialists Conference, 2004. PESC 04. 2004 IEEE 35th Annual, tomo 1, págs. 548 - 551 Vol.1 (2004b). ISSN 0275-9306.

Yang, S., A. Bryant, P. Mawby, D. Xiang, L. Ran, y P. Tavner. An industry-based survey of reliability in power electronic converters. Industry Applications, IEEE Transactions on, 47(3):1441-1451 (2011a). ISSN 0093-9994.

Yang, S., A. Bryant, P. Mawby, D. Xiang, L. Ran, y P. Tavner. An industry-based survey of reliability in power electronic converters. Industry Applications, IEEE Transactions on, 47(3):1441-1451 (2011b). ISSN 0093-9994.

Zargari, N., Y. Xiao, y B. Wu. A pwm csi-based vector controlled medium voltage ac drive with sinusoidal input and output waveforms. En Industry Applications Conference, 1997. Thirty-Second IAS Annual Meeting, IAS '97., Conference Record of the 1997 IEEE, tomo 1, págs. 768-774 vol.1 (1997). ISSN 0197-2618.

Zmood, D. y D. Holmes. A generalised approach to the modulation of current source inverters. En Power Electronics Specialists Conference, 1998. PESC 98 Record. 29th Annual IEEE, tomo 1, págs. 739 -745 vol.1 (1998). ISSN 0275-9306. 